\title{
A cavity-enhanced narrow-band multiphoton source for applications in quantum information
}

\author{
Reihaneh Shahrokhshahi \\ Tehran,Iran
}

M.S. Physics, Sharif University of Technology, 2006

\author{
A Dissertation presented to the Graduate Faculty \\ of the University of Virginia in Candidacy for the degree of \\ Doctor of Philosophy \\ Department of Physics \\ University of Virginia \\ August 2014
}




\section{Acknowledgments}

I would like to express my deepest gratitude to my advisor, Prof. Olivier Pfister, for his guidance and encouragement during my graduate studies. I have been fortunate to have an advisor who gave me the freedom and the confidence to explore on my own and taught me how to become an independent thinker. Beside all of the scientific support, I truly appreciate his invaluable comments, which provided me with priceless insights and prepared me for the academic world.

I would also like to give special thanks to all of my coworkers and collaborators, without whom I would not have been able to complete this work. First, I would like to thank Dr. Sae Woo Nam for providing us the opportunity to implement state of the art photon number resolving (PNR) detectors. I would also like to thank Prof. Aaron Miller for sharing his unique knowledge in operating PNR detectors, teaching us how to set up and operate PNR detectors, and initiating data acquisition and processing programs in our lab. Special appreciation also goes to Dr. Saikat Guha for his great ideas and advice in theoretical studies of quantum information. Also, I thank my colleague Niranjan Sridhar who assisted me in completing the optical reading experiment.

I also want to thank my fellow group members, Moran Chen, Niranjan Sridhar, Pei Wang and Wenjiang Fan, for great scientific discussions and also for being very good friends during all these years, which made the graduate program more productive and 
more enjoyable. I also thank the former member of the group Dr. Matthew Pysher, who generously taught me many basics of optical experiments.

Last and not least, I want to share my great gratitude to my parents, who have suffered but happily accepted to be so far apart from me all these years, without whose love and support none of this would have been possible. I would also like to give my very special thanks to my husband, Mohammad, for his extraordinary support and encouragement during all these years of study. 


\section{Abstract}

This thesis presents experimental progress towards highly efficient generation of cavityenhanced, narrow-band multiphotons using spontaneous parametric downconversion. It also suggests theoretical proposals for new applications of multiphoton sources in quantum information technology.

Photons prepared in Fock states, with a well-defined number of particles, are the essence of the quantum nature of light, and their unique nonclassical properties help us to develop quantum information technology. The generation of Fock states has been most commonly achieved by using spontaneous parametric down-conversion (SPDC) through the $\chi^{(2)}$ nonlinearity in bulk crystals. During the SPDC process the nonlinear crystal emits photon-number correlated modes, and a photon-numberresolving measurement in one mode heralds the preparation of the other mode in a Fock state. The fidelity and the success rate of Fock state generation is limited by the multimode nature of SPDC emission. In this thesis, I will investigate the use of cavity-enhanced SPDC modes for higher fidelity photon pair generation, in which the well defined mode of the cavity is enhanced and results in the narrow-band source of the heralded single-photons with up to $80 \%$ heralding efficiency. The heralding efficiency is defined as the ratio between number of coincidences and single events on the heralding mode. The photon-number-resolving ability of high-quantum-efficiency transition edge sensors is used for the heralding and detection. We further discuss the feasibility of Fock state generation with higher photon numbers upon improving 
the data acquisition and analysis techniques. We also demonstrate and implement an interferometry scheme that exploit the phase of the photon and is capable of reading boundless bits of digital information using a single photon.

This thesis also introduces new theoretical proposals for using Fock states to obtain quantum advantages for various quantum information applications, here to increase the efficiency in reading out information stored in a classical digital memory and to discriminate optical phases with lower error rates than is feasible with classical protocols. 


\section{Contents}

Acknowledgments . . . . . . . . . . . . . . . . i

Abstract ............................... ii

$\begin{array}{lll}1 & \text { Introduction to quantum optics } & \mathbf{1}\end{array}$

1.1 Quantum states of light . . . . . . . . . . . . . . . . . 2

1.2 Classical and Nonclassical States of Light . . . . . . . . . . . . . . . . 8

1.2.1 Quantum degree of second-order coherence $g^{(2)}(0)$ for classical and nonclassical states of light . . . . . . . . . . . . . 9

1.3 Quantum optics and quantum interferometry . . . . . . . . . . . . . . 14

1.3 .1 Mach-Zehnder Interferometer . . . . . . . . . . . . . . . . . . 15

1.3 .2 Heisenberg limited quantum interferometry. . . . . . . . . . . 19

2 Cavity enhanced single-photon source 25

2.1 Introduction . . . . . . . . . . . . . . . . . . . . . . . 25

2.2 Photon-number-resolving transition-edge-sensors . . . . . . . . . . . 29

2.3 Heralded single photon source . . . . . . . . . . . . . . . . 33

$2.3 .1 \quad$ Experimental setup . . . . . . . . . . . . . . . 34

2.3 .2 Crystal polarimetry . . . . . . . . . . . . . . . . . . . . . . . . 38

2.3 .3 Filtering Cavity . . . . . . . . . . . . . . . . . . 45

$2.4 \quad$ Cavity enhanced and high-heralding-ratio single-photon source . . . . 50

$2.4 .1 \quad$ Input-output formulation for optical cavities . . . . . . . . . . 52

2.4 .2 Experimental observation of the cavity enhancement and the onset of stimulated emission in an OPO operating well below the oscillation threshold . . . . . . . . . . . . . . . . . . 64

2.5 Conclusion . . . . . . . . . . . . . . . . . . . . 86

$\begin{array}{lll}3 & \text { Fock State Interferometry } & 88\end{array}$

3.1 Introduction . . . . . . . . . . . . . . . . . . . . . . . . . . . . . . . . 88 
3.2 Holland-Burnett Interferometer . . . . . . . . . . . . . . . . . . . . . 91

3.2 .1 The Schwinger Representation . . . . . . . . . . . . . . . . . 93

3.2 .2 Measurement method . . . . . . . . . . . . . . . . . . . . . . . 99

3.3 MZI for phase discrimination $\ldots \ldots \ldots \ldots$

3.4 Information theory $\ldots \ldots \ldots \ldots \ldots \ldots$

3.5 Optical Phase Discrimination $\ldots \ldots \ldots$

$3.5 .1 \quad$ Binary phase discrimonation, $\mathrm{M}=2 \ldots \ldots \ldots$

$3.5 .2 \quad 3$-ary phase discrimination, $\mathrm{M}=3$. . . . . . . . . . . . . 119

3.6 Optical Reading . . . . . . . . . . . . . . . . . . . . . . . . . . . . . 127

3.7 Conclusion . . . . . . . . . . . . . . . . . . . . . . . . . . . . . . 134

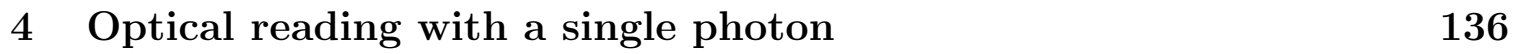

4.1 Introduction . . . . . . . . . . . . . . . . . . . . . . 136

4.2 Principle of the experiment $\ldots \ldots \ldots \ldots \ldots \ldots$

4.2 .1 Interferometer . . . . . . . . . . . . . . . . . . . . . . . . 139

4.3 Single-photon source $\ldots \ldots \ldots \ldots$

4.3.1 Quantum degree of second-order coherence $g^{(2)}(0)$ for heralded single-photon source . . . . . . . . . . . . . . . 142

4.4 Interferometer Performance $\ldots \ldots \ldots$

4.4 .1 Interferometer with coherent state input . . . . . . . . . 146

4.4 .2 Interferometry with heralded single photon source . . . . . . . 147

4.4 .3 Photon Information Efficiency . . . . . . . . . . . . . . 150

4.5 Conclusion $\ldots \ldots \ldots \ldots \ldots \ldots \ldots$

$\begin{array}{lll}5 \text { Conclusions } & 155\end{array}$

\begin{tabular}{|l|l|l|}
\hline A Multipartite Entanglement in the optical parametric oscillator & 158
\end{tabular}

\begin{tabular}{ll}
\hline B Data Acquisition and Data Analysis & 176
\end{tabular} 


\section{List of Figures}

1.1 Squeezed and coherent state ellipses in phase space . . . . . . . . . . 8

1.2 Schematic of the MZI. . . . . . . . . . . . . . . . . . . . . . . . . . . 15

1.3 Schematic of the BS. . . . . . . . . . . . . . . . . . . 17

2.1 Multiphoton generation . . . . . . . . . . . . . . . 26

2.2 Principle of TES operation $\ldots \ldots \ldots$. . . . . . . . . . . . 30

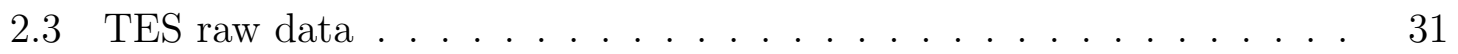

2.4 A histogram of TES peak sums . . . . . . . . . . . . . . . 32

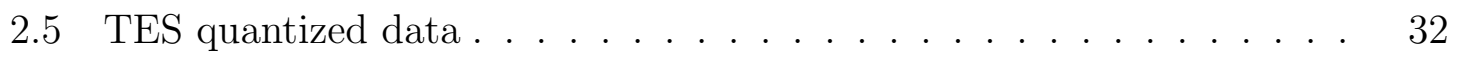

2.6 Experimental setup . . . . . . . . . . . . . . . . . . . . . . . 35

2.7 signal level vs fiber core position . . . . . . . . . . . . . . . . . . 36

2.8 Walk off in birefringent crystals . . . . . . . . . . . . . . . . . . . . . 38

2.9 Crystal fringes generated in birefringent crystals . . . . . . . . . . . . 40

2.10 Crystal polarimetry on HGKTP crystals . . . . . . . . . . . . . . . 41

2.11 Compensating for crystal walk off . . . . . . . . . . . . . . . . . 42

2.12 Crystal fringes for compensated crystal . . . . . . . . . . . . . . . . 43

2.13 Multmode SPDC emission . . . . . . . . . . . . . . . . . . . . 44

2.14 Phase mismatched SPDC emission. . . . . . . . . . . . . . . . . . . 45

2.15 Experimental set up with filtering cavity . . . . . . . . . . . . . 46

2.16 SPDC frequency spectrum . . . . . . . . . . . . . . . . . . . . . 47

2.17 Cavity spectrum for seed and locking laser . . . . . . . . . . . . . . . 48

2.18 Photon-number correlation vs filtering cavity detuning . . . . . . . . 49

2.19 A schematic representation of the cavity field. . . . . . . . . . . . . 52

2.20 Intensity of the resonant SPDC field transmitted by the cavity, versus time . . . . . . . . . . . . . . . . . 60

2.21 Schematic of the Optical Parametric Oscillator (OPO) . . . . . . . 65

2.22 Doubly resonant cluster structure for OPO above the oscillation threshold 68 
2.23 Doubly resonant cluster structure for OPO below the oscillation threshold 69

2.24 Comparison of the doubly resonant cluster structures for OPOs below and above the oscillation threshold . . . . . . . . . . . . . . . . . 70

2.25 Comparison of the doubly resonant cluster structures for OPOs below and above the oscillation threshold, zoomed in . . . . . . . . . . . . . 71

2.26 Cluster mode structure and its time constants . . . . . . . . . . . . . 72

2.27 Time constants for doubly resonant cluster mode structures below and above the oscillation threshold . . . . . . . . . . . . . . . 73

2.28 Stimulated emission for various pump powers . . . . . . . . . . . . . 74

2.29 Constant signal rate vs cavity length tuning, no sign of doubly resonant effect. . . . . . . . . . . . . . . . . . 76

2.30 Frequency spectrum of singly resonant modes . . . . . . . . . . . . . 77

2.31 Frequency spectrum of double resonant modes . . . . . . . . . . . . . 78

2.32 Frequency spectrum of filtered double resonant modes . . . . . . . . . 79

2.33 Experimental setup with OPO and filtering cavity . . . . . . . . . . . 80

2.34 Doubly resonant modes on TES . . . . . . . . . . . . . . . . 81

2.35 Doubly resonant modes on TES, zoomed in . . . . . . . . . . . . . . 82

2.36 Number of heralding and heralded photons along with the heralding ratio on precise time intervals . . . . . . . . . . . . . . . 83

2.37 Correlation function . . . . . . . . . . . . . . . . . 85

3.1 Imaging setup . . . . . . . . . . . . . . . . . . . . . . . . . . . . . . . 90

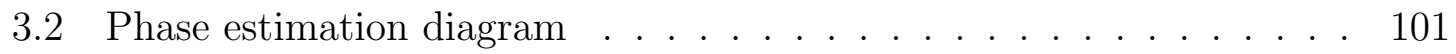

3.3 Selection rule . . . . . . . . . . . . . . . . . . . . . . . . . . . 103

3.4 The Mathematica program . . . . . . . . . . . . . . . 106

$3.5 \quad$ Probability of error Pe vs $p$ for optical phase discrimination between two phases . . . . . . . . . . . . . . . . 113

$3.6 \quad$ Probability of error (Pe) vs $p$ for optical phase discrimination for $M=2$ and for various $\mu \ldots \ldots \ldots \ldots 114$

$3.7 \quad$ Optimum p vs $N_{a v g}, M=2 \ldots \ldots \ldots \ldots$. . . . . . . . . . 115

3.8 Pe vs $N=N_{\text {avg }}, M=2$, for various experimental schemes . . . . . 118

$3.9 \quad$ Probability of error (Pe) vs phase shifts, $M=3$. . . . . . . . . . . . 120

3.10 Probability of error (Pe) vs phase shifts, $M=3, \mu=1,2$. . . . . . . 121

3.11 Minimum Probability of error (Pe) and the Mutual Information (MI) for optimal phases and various input states . . . . . . . . . . . . . . . 122 
3.12 Minimum Probability of error (Pe) vs $\mathrm{N}$ for $M=3$, and for all forms of input states . . . . . . . . . . . . . . . . . . . . . . . . . . . . . 123

3.13 The selection rule chart for discriminating between $M=4$ optical phase shifts, and for $|3,0\rangle_{z}$ input spin . . . . . . . . . . . . . 125

3.14 Optical phase discrimination for $\mathrm{M}=4,6$. . . . . . . . . . . . . . . . 126

3.15 Optical reading scheme . . . . . . . . . . . . . . . . . . . . . . . . . 128

3.16 The mutual information for optical reading, $n=1,2$. . . . . . . . . . . 129

3.17 Photon information efficiency $C\left(N_{s}\right) / N_{s}$ versus $N_{s}$, for various interferometry schemes. . . . . . . . . . . . . . . . . . . . 131

3.18 Photon information efficiency vs the encoding efficiency . . . . . . . 132

3.19 Optimum $\mathrm{p}$ for the MZI interferometer fed with two different effective spins. . . . . . . . . . . . . . . . . . . . . 133

3.20 The capacity of optical reading using Fock states with 3-ary PSK modulated phase encoding. . . . . . . . . . . . . . . . . . . . . . . . . . 134

$4.1 \quad$ Principle of high-PIE optical reading scheme . . . . . . . . . . . . . . 138

4.2 Polarization implementation of 4-port interferometer with only two optical paths. . . . . . . . . . . . . . . . . . . . . . . . . . . . . . . . 140

4.3 Raw TES data over $13 \mathrm{~ms}$. . . . . . . . . . . . . . . . . . . . . . . . 143

4.4 Effect of imperfect detection on the value of $g^{(2)}(0)$. . . . . . . . . 145

4.5 Interference fringes, coherent state input . . . . . . . . . . . . . . . . 148

4.6 Interference fringes, single photon input. . . . . . . . . . . . . . . . . 149

4.7 PIE vs interference visibility $\ldots \ldots \ldots$. . . . . . . . . . . . . . 154 


\section{Chapter 1}

\section{Introduction to quantum optics}

The nature of light has been established by quantum mechanics to be both wave and particle, and contradictory points of view- localized particle like nature and delocalized field nature- can coexist. This duality addresses the inability of the classical concepts, particle or wave, to fully describe the behavior of the quantum light.

Many aspects of light can be described by classical optics, which considers light as an electro magnetic wave. But classical optics is not complete and cannot describe fascinating experimental observations, such as blackbody radiation and photoelectric effect.

To explain these experimental observations, Albert Einstein gradually developed the concept of photons as discrete units of light with a certain frequency $\nu$, and each with energy $E=h \nu$. A beam of light contains a large numbers of photons, but cannot be described as a stream of photons traveling at the speed of light.

Quantum optics describes photons as an elementary excitation of the quantized electromagnetic field. Each field mode has energy states like a harmonic oscillator 
and the state of excitation of a mode is said to be the number of photons in a mode, so photons are distributed through the mode and are not localized to any specific position like particles.

Here, we introduce quantum states of the quantized electromagnetic field which are appropriate to the description of optical fields. We first describe number or Fock states, these states contains a well-defined number of photons. We then talk about optical fields which involve a superposition of number states. Such coherent states of the light are the closest possible quantum representation of a classical field.

\subsection{Quantum states of light}

The Hamiltonian of a quantized electromagnetic field, with frequency $\omega_{k}$ and traveling in free space, is

$$
H=\sum_{k} \hbar \omega_{k}\left(a_{k}^{\dagger} a_{k}+\frac{1}{2}\right)
$$

where $a_{k}$ and $a_{k}^{\dagger}$ are mutually adjoint operators which follow the bosonic commutation relations:

$$
\left[a_{k}, a_{k^{\prime}}\right]=\left[a_{k}^{\dagger}, a_{k^{\prime}}^{\dagger}\right]=0 \quad\left[a_{k}, a_{k^{\prime}}^{\dagger}\right]=\delta_{k k^{\prime}}
$$

These operators are annihilation and creation operators, where $a_{k}$ annihilates and $a_{k}^{\dagger}$ 
creates a photon with a wave vector $k$, where:

$$
\begin{aligned}
a_{k}\left|n_{k}\right\rangle & =\sqrt{n_{k}}\left|n_{k}-1\right\rangle \\
a_{k}^{\dagger}\left|n_{k}\right\rangle & =\sqrt{n_{k}+1}\left|n_{k}+1\right\rangle
\end{aligned}
$$

The Hamiltonian represents the sum of the number of photons in each mode $k$ multiplied by the energy of the photon $\hbar \omega_{k}$ in that mode. The eigenvalues of the Hamiltonian are $\hbar \omega_{k}\left(n_{k}+1 / 2\right)$, where $n_{k}$ is an integer and represents the number of photons in each mode $k$. The photon number operator is then

$$
N_{k}=a_{k}^{\dagger} a_{k}
$$

\section{Fock states}

Fock states or number states are quantum states of light with constant photon numbers. These are eigenstates of the number operator $N_{k}$,

$$
N_{k}\left|n_{k}\right\rangle=a_{k}^{\dagger} a_{k}\left|n_{k}\right\rangle=n_{k}\left|n_{k}\right\rangle
$$

which can be generated from the vacuum state by successive application of the creation operator

$$
\left|n_{k}\right\rangle=\frac{\left(a_{k}^{\dagger}\right)^{n_{k}}}{\left(n_{k} !\right)^{1 / 2}}|0\rangle
$$

Number states are orthogonal $\left\langle n_{k} \mid n_{l}\right\rangle=\delta_{k l}$, and complete

$$
\sum_{n_{k}=0}^{\infty}\left|n_{k}\right\rangle\left\langle n_{l}\right|=\mathbb{1}
$$


They form a complete basis for a Hilbert space.

At this point we only consider single mode quantum states with a well-defined frequency $\omega_{k}$, and drop the $k$ from equations. Fock states are purely quantum mechanical and have no classical counterpart and are the essence of the quantum nature of the light. However, it is extremely difficult to create higher photon number Fock states.

\section{Coherent states}

Coherent states of light have practical significance in quantum optics as a highly stabilized laser operating above threshold generate coherent states. They are eigenstates of the creation operator:

$$
a|\alpha\rangle=\alpha|\alpha\rangle
$$

and can be generated by applying the displacement operator,

$$
D(\alpha)=e^{\alpha a^{\dagger}-\alpha^{*} a}
$$

to the vacuum state, where $\alpha$ is a complex number. This yields [1]:

$$
|\alpha\rangle=D(\alpha)|0\rangle=e^{-|\alpha| / 2} \sum_{n=0}^{\infty} \frac{\alpha^{n}}{(n !)^{1 / 2}}|n\rangle
$$

Therefore, the probability of obtaining a given photon number in a coherent state is given by a Poisson distribution,

$$
P(n)=|\langle n \mid \alpha\rangle|^{2}=\frac{|\alpha|^{2 n}}{n !} e^{-|\alpha|^{2}}
$$


with the average photon number,

$$
\langle N\rangle=|\alpha|^{2}
$$

The uncertainty in the measurement outcome of an observable $O$, also known as the noise of the operator $O$, is equivalent to the standard deviation defined as:

$$
\Delta O=\left(\left\langle O^{2}\right\rangle-\langle O\rangle^{2}\right)^{1 / 2}
$$

Therefore, the uncertainty in the photon number for a coherent state can be calculated as:

$$
\Delta N=\sqrt{\left\langle\left(a^{\dagger} a\right)^{2}\right\rangle-\left\langle\left(a^{\dagger} a\right)\right\rangle^{2}}=\sqrt{|\alpha|^{4}+|\alpha|^{2}-|\alpha|^{4}}=|\alpha|
$$

which is proportional to the average photon number, Eq. (1.13),

$$
\Delta N=|\alpha|=\sqrt{\langle N\rangle}
$$

and is referred to as the shot noise limit.

The annihilation and creation operators are not Hermitian and therefore are not observables. It is, however, possible to construct a pair of Hermitian operators from creation and annihilation operators

$$
\begin{aligned}
& Q=a+a^{\dagger} \\
& P=i\left(a^{\dagger}-a\right)
\end{aligned}
$$

Where $Q$ is the amplitude quadrature operator, and $P$ is the phase quadrature op- 
erator, they respectively correspond to the position and momentum of a harmonic oscillator. While they satisfy the canonical commutation relation,

$$
[Q, P]=2 i
$$

The corresponding Heisenberg inequality is

$$
\Delta Q \Delta P \geq 1
$$

The noises of the $Q$ and $P$ quadratures for a coherent state $|\alpha\rangle$ are

$$
\begin{gathered}
\Delta Q=\left(\left\langle\alpha\left|a^{2}+a^{\dagger} a+a a^{\dagger}+a^{\dagger^{2}}\right| \alpha\right\rangle-\alpha^{2}-\alpha^{* 2}-2|\alpha|^{2}\right)^{1 / 2}=1 \\
\Delta P=\left(\left\langle\alpha\left|-a^{2}+a^{\dagger} a+a a^{\dagger}-a^{\dagger^{2}}\right| \alpha\right\rangle+\alpha^{2}+\alpha^{* 2}-2|\alpha|^{2}\right)^{1 / 2}=1
\end{gathered}
$$

Therefore coherent state $|\alpha\rangle$ is the minimum uncertainty state for the $Q$ and $P$ quadratures, and satisfies $\Delta Q \Delta P=1$. They are the quantum states closest to a classical field.

\section{Squeezed states}

We can define a general class of minimum uncertainty states. These states have minimum uncertainty $\Delta Q \Delta P=1$, but they may have less noise in one quadrature say $\Delta Q<1$ and thus more noise in other quadrature $\Delta P>1$. This more general class of minimum uncertainty states are called squeezed states and can be generated by applying the squeezing operator

$$
S(\epsilon)=e^{\left(\epsilon^{*} a^{2}-\epsilon a^{\dagger^{2}}\right) / 2}
$$


where $\epsilon=r e^{2 i \phi}$. One can calculate the effect of squeezed operators on quadratures as [1],

$$
S^{\dagger}(\epsilon)\left(Q_{2}+i P_{2}\right) S^{\dagger}(\epsilon)=Q_{2} e^{-r}+i P_{2} e^{r}
$$

and quadrature noises are,

$$
\Delta Q_{2}=e^{-r} \quad \Delta P_{2}=e^{r}
$$

where $Q_{2}$ and $P_{2}$ are the rotated complex amplitudes, $\left(Q_{2}+i P_{2}\right)=(Q+i P) e^{-i \phi}$, and $\phi$ is the squeezing angle. Therefore, the squeezing operator attenuates one component of the rotated complex amplitude $Q_{2}$ and amplifies the other one $P_{2}$. The degree of attenuation and amplification is determined by the squeezing factor $r=|\epsilon|$.

A squeezed state is represented by an error ellipse in phase space, whose axis are $Q$ and $P$, Fig. 1.1. Increasing the squeezing factor stretches the ellipse. It should be compared to a coherent state, which is represented by an error circle in phase space.

The squeezed state $|\alpha, \epsilon\rangle$ is obtained by first squeezing the vacuum and then displacing it.

$$
|\alpha, \epsilon\rangle=D(\alpha) S(\epsilon)|0\rangle
$$

The average photon number for the squeezed state is:

$$
\langle N\rangle=|\alpha|^{2}+\sinh ^{2} r
$$

The average photon number for the squeezed vacuum, $\alpha=0$ is not zero and increases 


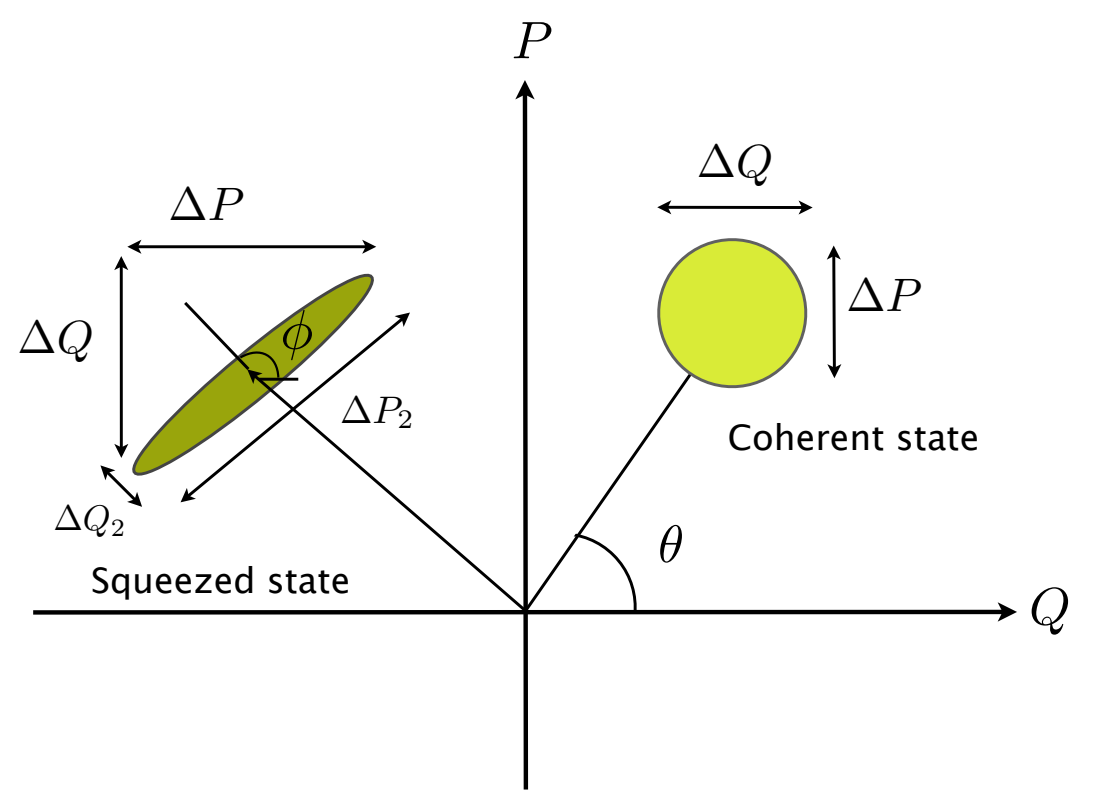

Figure 1.1: The noise for the quadrature $Q$ and phase $P$, for coherent and squeezed states. $\phi$ is the squeezing angle, and $\Delta Q_{2}$ and $\Delta P_{2}$ are rotated quadratures.

with the squeezing parameter,

$$
\langle N\rangle=\sinh ^{2} r
$$

The photon number uncertainty is then,

$$
\Delta N=\sqrt{\left\langle N^{2}\right\rangle-\langle N\rangle^{2}}=\left(|\alpha|^{2} e^{-2 r}+2 \sinh ^{2} r \cosh ^{2} r\right)^{1 / 2}
$$

which should be compared to the shot noise limit Eq. 1.16.

\subsection{Classical and Nonclassical States of Light}

So far we have described three different states of quantized light, the Fock state, the coherent state and the squeezed state. Coherent states are closest to classical light, but Fock and squeezed states have nonclassical nature and cannot be described by 
classical optics. These nonclassical light states have nonclassical noise properties, called quantum noise, and will be discussed later in section 1.3 .

Fluctuations and coherence properties of nonclassical light cannot be described in classical terms. The second order of coherence plays a crucial role in distincting between a classical and nonclassical states of light.

In the following we calculate the second-order correlation function for zero time delay, $g^{(2)}(0)$, and divide different states of light into classical and nonclassical states based on their second order correlation function.

\subsubsection{Quantum degree of second-order coherence $g^{(2)}(0)$ for classical and nonclassical states of light}

In optics, correlation functions are used to characterize statistical properties of the electromagnetic field [1]. The degree of coherence is the normalized correlation of electric fields. Properties of the light beam that are relevant to optical interference can be expressed in terms of the concept of the optical coherence. Light at two points in space or time that is capable of being superimposed to produce interference is said to be coherent and the potential magnitude of the interference effects is governed by the first-order coherence of the light beam employed.

The degree of first-order coherence is defined as the normalized electric field correlation function:

$$
g^{(1)}\left(r_{1}, t_{1}, r_{2}, t_{2}\right)=\frac{\left\langle E^{-}\left(r_{1}, t_{1}\right) E^{+}\left(r_{2}, t_{2}\right)\right\rangle}{\left(\left\langle E^{-}\left(r_{1}, t_{1}\right) E^{+}\left(r_{1}, t_{1}\right)\right\rangle\left\langle E^{-}\left(r_{2}, t_{2}\right) E^{+}\left(r_{2}, t_{2}\right)\right\rangle\right)^{1 / 2}},
$$


where

$$
\begin{aligned}
& E^{+}(r, t)=i \sum_{k}\left(\hbar w_{k} / 2 \epsilon_{0} V\right)^{1 / 2} \varepsilon_{k} a_{k} \exp \left(-i w_{k} t+i \vec{k} \cdot \vec{r}\right) \\
& E^{-}(r, t)=-i \sum_{k}\left(\hbar w_{k} / 2 \epsilon_{0} V\right)^{1 / 2} \varepsilon_{k} a_{k}^{\dagger} \exp \left(i w_{k} t-i \vec{k} \cdot \vec{r}\right)
\end{aligned}
$$

Angle brackets denote statistical averages.

Differences between classical and quantum predictions for first-order interference experiments tend to be difficult to detect. In fact, both the classical and quantum degrees of first-order have numerical value in the same range,

$$
0 \leq\left|g^{(1)}(\tau)\right| \leq 1
$$

More striking differences occur in measurements that depend upon the degree of second-order coherence, which is used to find the statistical character of intensity fluctuations. All the existing experiments that distinguish classical from quantum properties belong to this category. The degree of second-order coherence is defined in terms of the correlation of light intensities at two space time points $\left(r_{1}, t_{1}\right)$ and $\left(r_{2}, t_{2}\right):$

$$
g^{(2)}\left(r_{1}, t_{1}, r_{2}, t_{2} ; r_{2}, t_{2}, r_{1}, t_{1}\right)=\frac{\left\langle E^{-}\left(r_{1}, t_{1}\right) E^{-}\left(r_{2}, t_{2}\right) E^{+}\left(r_{2}, t_{2}\right) E^{+}\left(r_{1}, t_{1}\right)\right\rangle}{\left\langle E^{-}\left(r_{1}, t_{1}\right) E^{+}\left(r_{1}, t_{1}\right)\right\rangle\left\langle E^{-}\left(r_{2}, t_{2}\right) E^{+}\left(r_{2}, t_{2}\right)\right\rangle}
$$

All of the factors in Eq. 11.30, Eq. (1.34) are expectation values of products of Hermitian operators with their conjugates and must be positive

$$
0 \leq g^{(2)}(0) \leq \infty
$$


However, If the electric fields are considered classical, we can reorder them to express $g^{(2)}(0)$ in terms of intensities, $I\left(r_{1}, t_{1}\right)=E^{+}\left(r_{1}, t_{1}\right) E^{-}\left(r_{1}, t_{1}\right)$.

For the classical light

$$
\left\langle E^{-}\left(r_{1}, t_{1}\right) E^{-}\left(r_{1}, t_{1}\right) E^{+}\left(r_{1}, t_{1}\right) E^{+}\left(r_{1}, t_{1}\right)\right\rangle=\left\langle I^{2}\right\rangle
$$

then

$$
g^{(2)}(0)=\frac{\left\langle I^{2}\right\rangle}{\langle I\rangle^{2}}
$$

And by means of Cauchy's inequality, one can show that

$$
\left(\frac{\sum_{i}^{N} I_{i}}{N}\right)^{2} \leq \frac{\sum_{i}^{N} I_{i}{ }^{2}}{N} \longrightarrow\langle I\rangle^{2} \leq\left\langle I^{2}\right\rangle
$$

Therefore, if the light is classical then $g^{2}(0)$ must satisfy

$$
g^{(2)}(0) \geq 1
$$

And there is a nonclassical range of values for which

$$
0 \leq g^{(2)}(0) \leq 1
$$

This leads to significant differences between quantum and classical second-order coherence functions. Here, we calculate $g^{(2)}(0)$ for the coherent and thermal sources and compare it with $g^{(2)}(0)$ of Fock state.

A considerable simplification in the computation of the degree of second order coherence occurs when the light beam exists only in a single mode of the optical 
system. Most factors in Eq. 1.34 cancel and $g^{(2)}(0)$ simplifies to,

$$
g^{(2)}(0)=\frac{<a^{\dagger} a^{\dagger} a a>}{<a^{\dagger} a>^{2}}=\frac{<a^{\dagger} a a^{\dagger} a>-<a^{\dagger} a>}{<a^{\dagger} a>^{2}}
$$

Defining $N=a^{\dagger} a$,

$$
g^{(2)}(0)=\frac{<N^{2}>-<N>}{<N>^{2}}
$$

Hanbury Brown and Twiss [2], [3], proposed and implemented a method to observe second order coherence using an intensity interferometer. We determined the photon number statistics and the average photon numbers using a photon-number-resolving PNR detector, and directly estimating $g^{(2)}(0)$ without running the Hanbury Brown and Twiss experiment.

To determine the photon number averages, we looked at detector data over duration $\Delta t$, then divided it into smaller time bins of size $\delta t$, which is in the order of the detector resolution or cool down time, then calculated the average photon number in each time bin. The total number of bins is

$$
N_{\text {bins }}=\frac{\Delta t}{\delta t}
$$

If we measure $m_{i}$ photons per $\delta t$, then,

$$
\begin{aligned}
& <N>=\frac{1}{N_{\text {bins }}} \sum_{i} m_{i} \\
& <N^{2}>=\frac{1}{N_{\text {bins }}} \sum_{i} m_{i}^{2}
\end{aligned}
$$


and

$$
g^{(2)}(0)=N_{\text {bins }} \frac{\sum_{i} m_{i}^{2}-\sum_{i} m_{i}}{\left(\sum_{i} m_{i}\right)^{2}}
$$

If we have $n_{1}$ one-photon peaks and $n_{2}$ two-photon peaks and no higher photon numbers during $\Delta t$, then we have $n_{0}=N_{\text {bins }}-n_{1}-n_{2}$ and

$$
\sum_{i} m_{i}=n_{1}+2 n_{2} \quad \sum_{i} m_{i}^{2}=n_{1}+4 n_{2}
$$

So,

$$
g^{(2)}(0)=N_{b i n s} \frac{n_{1}+4 n_{2}-\left(n_{1}+2 n_{2}\right)}{\left(n_{1}+2 n_{2}\right)^{2}}=N_{\text {bins }} \frac{2 n_{2}}{\left(n_{1}+2 n_{2}\right)^{2}}
$$

Therefore, one can easily calculate $g^{(2)}(0)$, knowing the photon number statistics on one and two photons. Note, here we consider very attenuated intensity beams and the probability of detecting more than two photons is very low and is taken to be zero.We rewrite $g^{(2)}(0)$ in terms of probabilities $p_{j}=n_{j} / N_{\text {bins }}$ to get

$$
g^{(2)}(0)=\frac{2 p_{2}}{\left(p_{1}+2 p_{2}\right)^{2}}
$$

As mentioned, this applies for very attenuated light. For a coherent state, it means $\alpha \rightarrow 0$. Plugging the photon number probabilities of a coherent state Eq. 1.12), $p_{n}=e^{-\alpha^{2}} \frac{\alpha^{2 n}}{n !}$, into Eq. 1.41 yields $g^{(2)}(0)=1$ for coherent sources. This proof is more general and is true for all values of $\alpha$ as,

$$
g^{(2)}(0)=\frac{\left\langle\alpha\left|a^{\dagger} a^{\dagger} a a\right| \alpha\right\rangle}{\left\langle\alpha\left|a^{\dagger} a\right| \alpha\right\rangle^{2}}=\frac{\alpha^{4}}{\alpha^{4}}=1
$$


Similarly for Fock states $\mid n>, g^{(2)}(0)$ simplifies to:

$$
g^{(2)}(0)=\frac{\left\langle n\left|a^{\dagger} a^{\dagger} a a\right| n\right\rangle}{\left\langle n\left|a^{\dagger} a\right| n\right\rangle^{2}}=\frac{n(n-1)}{n^{2}}=\frac{n-1}{n}
$$

Therefore $g^{(2)}(0)$ is less than one for Fock states, and reach its lowest value of zero for a single photon source. It is also expected from Eq. (1.48) when $n_{2}=0$.

It proves that Fock states have second order coherence values out of the range predicted by classical optics, Eq. 1.39 . We measured the $g^{(2)}(0)$ of a single photon in our lab and reported values less than one, as will be discussed in more detail, in section 4.3.1.

\subsection{Quantum optics and quantum interferometry}

One of the important applications of nonclassical light is precision measurement and interferometry, as nonclassical states of light can be used for ultra precise optical phase estimation.

The precision in optical measurements with coherent states of light that rely on interferometry are limited by the vacuum noise of beam splitters Eq. (1.16), but using quantum properties of light one can beat this "shot noise" level of beam splitters and attain more precise measurements over what is classically possible. In the case of optical interference measurements, this is referred to as Heisenberg limited interferometry (HLI).

To measure an optical phase one can use an interference experiment. The MachZehnder Interferometer (MZI) is an example of an interferometer. In the following, 
we study MZI and basic optical elements such as beam splitters and phase shifters in quantum optics, then will focus on optical interferometry with nonclassical light.

\subsubsection{Mach-Zehnder Interferometer}

The MZI is a two-mode light interferometer, Fig. 1.2, it consists of two beam splitters (BS) to separate and recombine fields. A path difference between arms of the MZI results in a phase shift $\phi$ between arms. The distribution of output beam intensities of the MZI depends on the optical phase difference between arms of the interferometer.

In order to control the optical phase shift one of the reflecting mirrors is mounted to a piezoelectric transducer (PZT), so that $\phi$ is controlled by applying a variable voltage to the PZT.

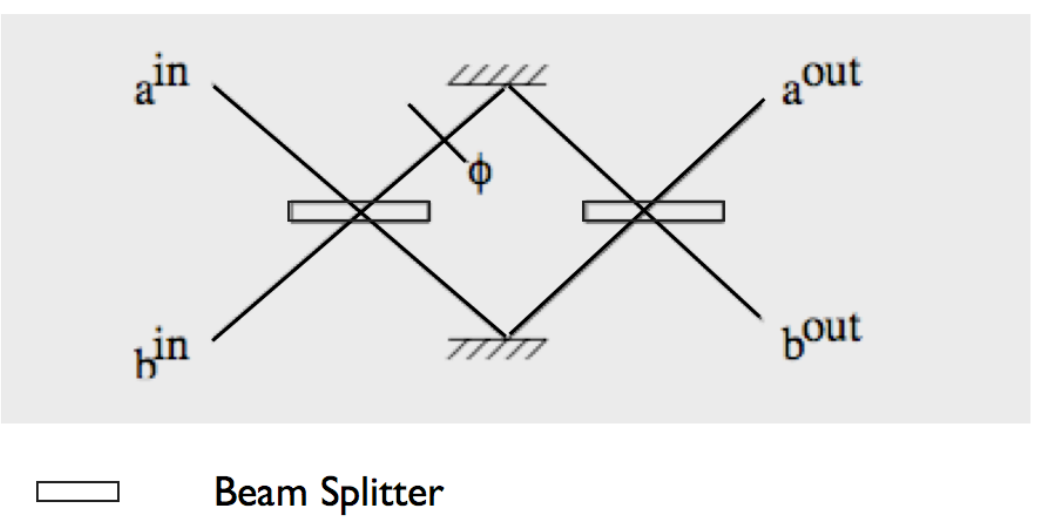

Figure 1.2: Schematic of the MZI. 
To study the evolution of the quantum state in the MZI, we first study the effect of its components, the beam splitter and phase shifter on a quantum state.

\section{Phase Shift Operator}

The evolution of quantum state is easier to compute in the Heisenberg picture, where operators are propagated and the state is static.

The Heisenberg equation of motion is,

$$
i \hbar \dot{a}(t)=[a(t), H(t)]
$$

Using the Baker-Haussdorff lemma, one can solve the Heisenberg equation as,

$$
a(t)=U^{\dagger}(t) a U(t)
$$

where $U=e^{\frac{-i}{\hbar} H t}$ is the unitary evolution operator.

Since a phase shift difference is equivalent to a path difference between two modes, then one can deduce the unity phase shift operator from the free Hamiltonian Eq. 11.1 as,

$$
U(\phi)=e^{-i \phi a^{\dagger} a}
$$

where $\phi=\omega t$ is an optical phase shift. The action of the phase shift operator is then,

$$
\begin{gathered}
U^{\dagger}(\phi) a U(\phi)=a e^{-i \phi} \\
a_{\text {out }}=e^{-i \phi} a
\end{gathered}
$$

The single mode phase shift operator can generalize to a relative phase shift operator 
between modes $a$ and $b$, and can be presented in a matrix form as,

$$
\left(\begin{array}{l}
a_{\text {out }} \\
b_{\text {out }}
\end{array}\right)=\left(\begin{array}{cc}
e^{i \phi / 2} & 0 \\
0 & e^{-i \phi / 2}
\end{array}\right)\left(\begin{array}{l}
a_{\text {in }} \\
b_{\text {in }}
\end{array}\right)
$$

where $a_{i n}, b_{i n}$ are input and $a_{\text {out }}, b_{\text {out }}$ are output quantum modes.

\section{Beam Splitter}

A beam splitter is a partially reflecting mirror, Fig. 1.3 , and its hamiltonian can be written as:

$$
H_{B S}=\hbar \kappa\left(a^{\dagger} b+a b^{\dagger}\right)
$$

The evolution operator is

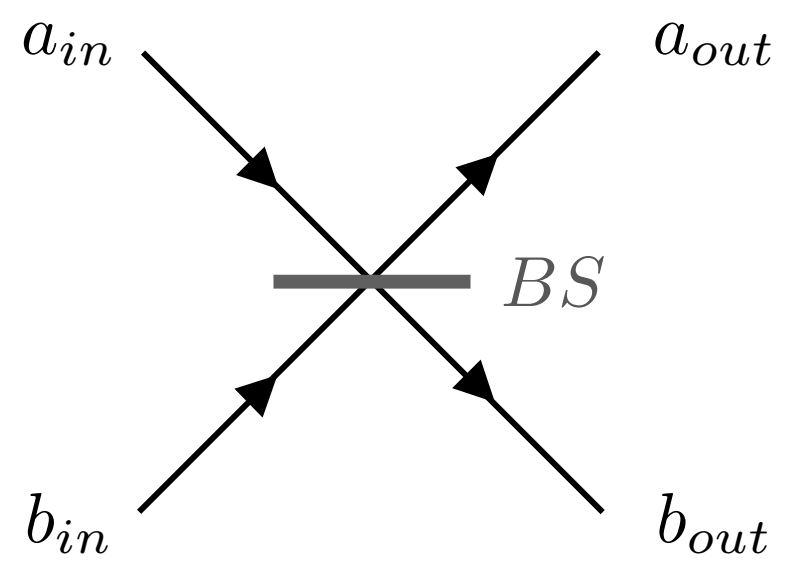

Figure 1.3: Schematic of the BS.

$$
U_{B S}=e^{-i \kappa t\left(a^{\dagger} b+a b^{\dagger}\right)}
$$

and its action in Heisenberg picture can be calculated as, 


$$
\left(\begin{array}{c}
a_{\text {out }} \\
b_{\text {out }}
\end{array}\right)=\left(\begin{array}{l}
a(t) \\
b(t)
\end{array}\right)=\left(\begin{array}{cc}
\cos \kappa t & i \sin \kappa t \\
i \sin \kappa t & \cos \kappa t
\end{array}\right)\left(\begin{array}{c}
a_{\text {in }} \\
b_{\text {in }}
\end{array}\right)
$$

Defining optical Fresnel coefficients of the dielectric layer on the beam splitter surface, as $\rho=\cos (k t)$ and $\tau=\sin (k t), \rho^{2}+\tau^{2}=1$, simplifies the matrix equation to,

$$
\left(\begin{array}{c}
a_{\text {out }} \\
b_{\text {out }}
\end{array}\right)=\left(\begin{array}{cc}
\rho & i \tau \\
i \tau & \rho
\end{array}\right)\left(\begin{array}{l}
a_{i n} \\
b_{\text {in }}
\end{array}\right)
$$

where

$$
U_{B S}=\left(\begin{array}{cc}
\cos \kappa t & i \sin \kappa t \\
i \sin \kappa t & \cos \kappa t
\end{array}\right)=\left(\begin{array}{cc}
\rho & i \tau \\
i \tau & \rho
\end{array}\right)
$$

Thus, quantum modes $a$ and $b$ evolve in a beam splitter as,

$$
\begin{gathered}
a_{\text {out }}=U_{B S}^{\dagger} a U_{B S}=\rho a-\tau b \\
b_{\text {out }}=U_{B S}^{\dagger} b U_{B S}=\rho a+\tau b
\end{gathered}
$$

\section{MZI}

Now we can analyze the MZI by calculating its representing matrix. It contains three parts, the first 50:50 beam splitter a phase shifter and the second 50:50 beam splitter, Fig. 1.2. The representing matrix can then be written as, 


$$
\frac{1}{\sqrt{2}}\left(\begin{array}{cc}
1 & i \\
i & 1
\end{array}\right)\left(\begin{array}{cc}
e^{i \frac{\phi}{2}} & 0 \\
0 & e^{-i \frac{\phi}{2}}
\end{array}\right) \frac{1}{\sqrt{2}}\left(\begin{array}{cc}
1 & i \\
i & 1
\end{array}\right)=i\left(\begin{array}{cc}
\sin \frac{\phi}{2} & \cos \frac{\phi}{2} \\
\cos \frac{\phi}{2} & -\sin \frac{\phi}{2}
\end{array}\right)
$$

Where the first and last matrices represent 50:50 beam splitters and the middle one represents the effect of $\phi$ relative phase shift.

At $\phi=\pi$ the resulting matrix is identical to a that of a perfectly reflecting mirror, and at $\phi=\pi / 2$ the MZI acts as a 50:50 beam splitter.

\subsubsection{Heisenberg limited quantum interferometry}

Quantum mechanics imposes a fundamental limit on the precise measurement of an optical phase shift. This fundamental limit is extracted from the time-energy Heisenberg inequality,

$$
\Delta E \Delta t \geq \hbar
$$

For a photon field of frequency $\omega$, we have $E=N \hbar \omega$ and $\phi=\omega t$, the time-energy inequality can be rewritten as the number-phase inequality,

$$
\Delta E \Delta t=\left(\frac{\Delta \phi}{\omega}\right)(\Delta N \hbar \omega) \geq \hbar \Longleftrightarrow \Delta N \Delta \phi \geq 1
$$

Therefore the number-phase Heisenberg uncertainty between the optical phase $\phi$ and the photon number $N$ bounds the phase precision to the limit of $\Delta \phi=\frac{1}{\Delta N}$. $\Delta N$ fluctuations are bounded by the mean photon number $\langle N\rangle$, as a result of the energy constraint [4]. The resulting precision limit for phase measurements is called 
the Heisenberg limit.

$$
\Delta \phi=\frac{1}{\langle N\rangle}
$$

The Heisenberg inequality Eq. 1.63) for modes a and b inside the MZI can be generalized to

$$
\Delta\left(\phi_{a}-\phi_{b}\right) \geq \frac{1}{\Delta\left(N_{a}-N_{b}\right)}
$$

However, conventional interferometers with light fed only into one input are not optimal and can not reach the Heisenberg limit, because vacuum fluctuations enter the unused port of the interferometer and amplify fluctuations. The phase measurement sensitivity of this system cannot exceed what is sometimes called the standard quantum limit, or more appropriately shot-noise limit (SNL) of the beam splitter,

$$
\Delta \phi=\frac{1}{\sqrt{\langle N\rangle}}
$$

where $\langle N\rangle$ is the average number of photons injected to the interferometer. Therefore, vacuum fluctuations degrade the interferometer performance by the factor of $\frac{1}{\sqrt{\langle N\rangle}}$. We now study the MZI fed with coherent and Fock states and give a rigorous derivation of such limits.

\section{Coherent state input}

The beam splitter output with a coherent state $|\alpha\rangle_{a}$ in one input mode and a vacuum $|0\rangle_{b}$ in other one can be calculated using Eq. (1.10), Eq. (1.61), 


$$
\begin{aligned}
U_{B S}|\alpha\rangle_{a}|0\rangle_{b} & =U_{B S} D(\alpha)|0\rangle_{a}|0\rangle_{b} \\
& =e^{-|\alpha|^{2} / 2} U_{B S} e^{\alpha a^{\dagger}}|0\rangle_{a}|0\rangle_{b} \\
& =e^{-|\alpha|^{2} / 2} \sum_{n=0}^{\infty} \frac{\alpha^{n}}{n !}\left(U_{B S} a^{\dagger} U_{B S}^{\dagger}\right)^{n} U_{B S}|0\rangle_{a}|0\rangle_{b} \\
& =e^{-|\alpha|^{2} / 2} \sum_{n=0}^{\infty} \frac{\alpha^{n}}{n !}\left(\rho a^{\dagger}+\tau b^{\dagger}\right)^{n}|0\rangle_{a}|0\rangle_{b} \\
& =e^{-|\alpha|^{2} / 2} e^{\rho \alpha a^{\dagger}} e^{\tau \alpha b^{\dagger}}|0\rangle_{a}|0\rangle_{b} \\
\longrightarrow \quad & U_{B S}|\alpha\rangle_{a}|0\rangle_{b}=|\rho \alpha\rangle_{a}|\tau \alpha\rangle_{b}
\end{aligned}
$$

knowing $U_{B S}|0\rangle|0\rangle=|0\rangle|0\rangle$. Thus, a beam splitter splits the coherent state $|\alpha\rangle$ into two coherent states $|\rho \alpha\rangle$ and $|\tau \alpha\rangle$. The fluctuations of the photon number difference of modes $a$ and $b$ after the first beam splitter can be calculated as:

$$
\begin{aligned}
\Delta\left(N_{a}-N_{b}\right)^{2} & =\Delta\left(N_{a}\right)^{2}+\Delta\left(N_{b}\right)^{2} \\
& =|\tau \alpha|^{2}+|\rho \alpha|^{2}=|\alpha|^{2} \\
\Longleftrightarrow \quad & \Delta\left(N_{a}-N_{b}\right)=|\alpha| \\
& =\sqrt{\left\langle N_{a}-N_{b}\right\rangle}
\end{aligned}
$$

So, the phase precision is shot noise limited Eq. 1.66) for a MZI fed with a coherent state.

We now consider nonclassical Fock-states of light as the input of the interferometer, in order to achieve the Heisenberg limited interferometry. 


\section{Fock-state input}

Fock states are good potential candidates for phase interferometry. However, injecting Fock states to only one input of the BS results in interference of the light with vacuum field and the binomial probability law. The beam splitter evolves quantum modes prepared in $|n\rangle_{a}|0\rangle_{b}$ as:

$$
\left|\psi_{\text {out }}\right\rangle_{a, b}=U_{B S}|n\rangle_{a}|0\rangle_{b}=U_{B S} \frac{\left(a^{\dagger}\right)^{n}}{\sqrt{n !}}|0\rangle_{a}|0\rangle_{b}=\frac{\left(U_{B S} a^{\dagger} U_{B S}^{\dagger}\right)^{n}}{\sqrt{n !}}|0\rangle_{a}|0\rangle_{b}=\frac{\left(\rho a^{\dagger}+\tau b\right)^{n}}{\sqrt{n !}}|0\rangle_{a}|0\rangle_{b}
$$

Therefore, $\left|\psi_{\text {out }}\right\rangle_{a, b}$ can be written as two Fock states in modes $a$ and $b$ with a binomial photon counting probability distribution:

$$
\left|\psi_{\text {out }}\right\rangle_{a, b}=\sum_{m=0}^{n}\left(\begin{array}{c}
m \\
n
\end{array}\right)^{1 / 2} \rho^{m} \tau^{n-m}|m\rangle|n-m\rangle
$$

Based on the well-known properties of the binomial distribution, we get $\left\langle N_{a}-\right.$ $\left.N_{b}\right\rangle \propto n / 2$ and $\Delta\left(N_{a}-N_{b}\right) \propto \sqrt{n}$. Therefore, the phase sensitivity is shot noise limited $\Delta \phi=\frac{1}{\sqrt{\langle N\rangle}}, n=\langle N\rangle$.

The essential point here $[5]$ is that the interferometer phase noise for a Fock state is the shot noise of the beam splitter, due to the vacuum interference, and has nothing to do with the input state noise.

Therefore, using a single-mode Fock-state does not enable us to reach to the 
Heisenberg limit. In fact, many theoretical and experimental efforts have been made to improve the optical phase measurement sensitivity and to reach the Heisenberg limit for phase precision [6], [7], [8].

In this thesis we will mostly focus on proposals based on preparing light on indistinguishable Fock states and simultaneously injecting them into both arms of the interferometer. Indistinguishable Fock states are twin-photon states with equal photon numbers and are prepared with the exact same frequencies and wavevectors,

$$
|\psi\rangle=\left|\hat{k}_{a}, \omega, \hat{\epsilon} ; n\right\rangle \otimes\left|\hat{k}_{b}, \omega, \hat{\epsilon} ; n\right\rangle
$$

\section{Twin Fock-state input, Hong-Ou-Mandel Interference}

We first consider a simpler case of a twin-Fock state, with $n=1$, this yields

$$
\begin{aligned}
\left|\psi_{\text {out }}\right\rangle_{a, b} & =U_{B S}|1\rangle_{a}|1\rangle_{b} \\
& =U_{B S} a^{\dagger} b^{\dagger}|0\rangle_{a}|0\rangle_{b} \\
& =\left(U_{B S} a^{\dagger} U_{B S}^{\dagger}\right)\left(U_{B S} b^{\dagger} U_{B S}^{\dagger}\right)|0\rangle_{a}|0\rangle_{b} \\
& =\left(\rho a^{\dagger}+\tau b^{\dagger}\right)\left(\tau a^{\dagger}-\rho b^{\dagger}\right)|0\rangle_{a}|0\rangle_{b} \\
& =\left[\left(\rho \tau\left(a^{\dagger^{2}}-b^{\dagger^{2}}\right)+\left(\tau^{2}-\rho^{2}\right) a^{\dagger} b^{\dagger}\right)\right]|0\rangle_{a}|0\rangle_{b} \\
& =\sqrt{2} \rho \tau\left(|2\rangle_{a}|0\rangle_{b}-|0\rangle_{a}|2\rangle_{b}\right)+\left(\tau^{2}-\rho^{2}\right)|1\rangle_{a}|1\rangle_{b}
\end{aligned}
$$

For the 50:50 BS, $\rho=\tau=\frac{1}{\sqrt{2}}$, so the last term vanishes and the output state becomes

$$
\left|\psi_{\text {out }}\right\rangle_{a, b}=\sqrt{2} \rho \tau\left(|2\rangle_{a}|0\rangle_{b}-|0\rangle_{a}|2\rangle_{b}\right)
$$


The probability of getting $|1\rangle_{a}|1\rangle_{b}$ is zero, due to the quantum interference which restricts photons to emerge together at the same port of the BS. This effect is called Hong-Ou-Mandel (HOM) interference and is at the heart of the quantum optics.

Photons are not randomly distributed after the beam splitter and will be bunched at one or the other output ports. Thus the noise in the photon number is maximized, $\langle N\rangle=2$, and correspondingly the phase noise is minimized, $\Delta \phi=\frac{1}{2}$, which should be compared to the shot noise limit of $\Delta \phi=\frac{1}{\sqrt{2}}$

In the generalized HOM effect, the BS is fed with twin-Fock states with higher photon numbers. It is easier to study the generalized HOM in the Schwinger representation as we will study in detail in chapter 3 .

It is known that, implementing twin-Fock states facilitates achieving the Heisenberg limit for phase measurement [9]. We consider these states for error free and high resolution optical phase discrimination, as will be discussed in chapter 3 . 


\section{Chapter 2}

\section{A cavity-enhanced, narrow-band, and single-mode Fock-state source}

\section{$2.1 \quad$ Introduction}

Single and multiphoton sources prepared in Fock states have been a subject of great interest.These states are the essence of the quantum nature of light and have been at the heart of fundamental tests of the quantum theory, such as Bell inequalities [10], [11] [12] [13], [14.

They are also required in the development of quantum information technology and have many applications in quantum cryptography [15], [16], 17] [18], quantum metrology [6], [7],quantum communication [19], 20] and linear optical quantum computing (LOQC) 21]. Moreover, Fock states have a nonpositive (hence non-Gaussian) Wigner function, which is crucial to continuous-variable quantum information, namely exponential speedup in quantum computing, [22], entanglement distillation [23], quantum error correction [24] and fault tolerant quantum computing [25], [26].

The generation of Fock states has been most commonly achieved by using spon- 
taneous parametric down-conversion (SPDC) in nonlinear crystals [27], [28]. During the SPDC process a pump photon is annihilated and lower energy signal and idler photons are emitted in:

$$
|\psi\rangle=\frac{1}{\cosh r} \sum_{n=0}^{\infty} \tanh ^{n} r|n\rangle_{\text {signal }}|n\rangle_{\text {idler }}
$$

Here, $\mathrm{r}$ is proportional to the nonlinear interaction and the pump field amplitude.

Detection of signal photons is used to herald idler photons as in Fig. 2.1.

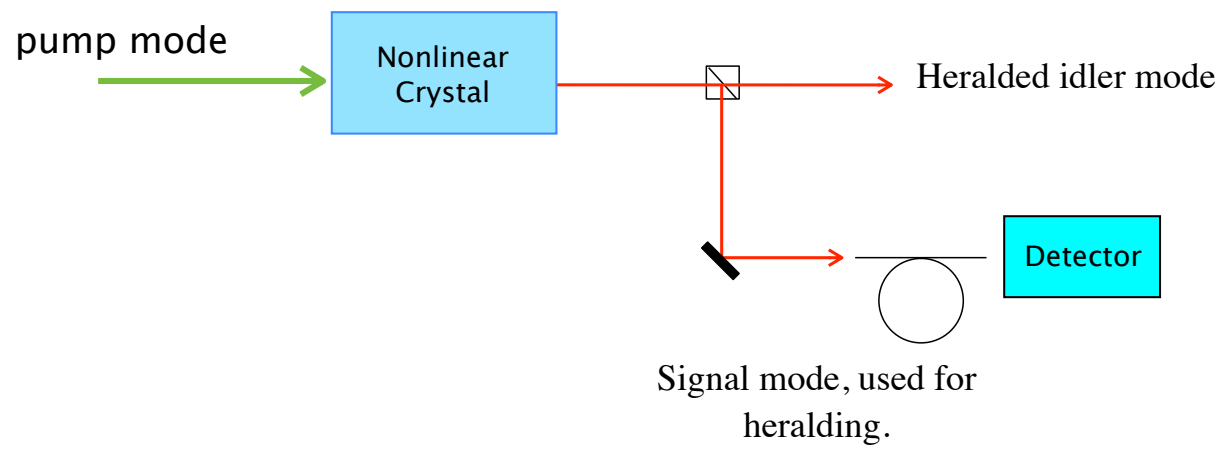

Figure 2.1: pump mode spontaneously down converts to lower-energy cross-polarized signal and idler modes These modes are always emitted with equal photon numbers and measuring $\mathrm{n}$ photons on the signal mode, $|n\rangle_{\text {signal }}$, would herald the existence of $\mathrm{n}$ photons on the idler mode, in $|n\rangle_{\text {idler }}$.

For low pump photon flux, small $r$, the multi-photon SPDC emission is negligible and photons are mostly emitted in pairs, so that a photon click on the signal detector conditionally prepares a single-photon state on the idler, as initially demonstrated by Lvovsky et al [29].

A more recently developed type of heralded single and multi-photon generation is based on spontaneous four-wave mixing in optical fibers 30 . In that scheme photon 
pairs can be generated directly in the guided mode of the optical fiber and results in all-fiber photon sources with low optical losses [31].

Single photons can also be generated on demand from single quantum emitters, such as in quantum dots [32], single atoms and ions [33], 34] and in Nitrogen-vacancy (NV) centers in diamond 35 .

In this chapter, I focus on single-photon generation using SPDC sources. The fidelity and the success rate of Fock state generation is limited by the multimode nature of SPDC photons. Currently, many experimental efforts are in progress to improve various aspects of single-photon sources. A big effort is particularly required towards improving the single-photon collection and detection efficiencies which is essential, for example, for loophole-free tests of Bell's inequality [10]. Many tests of Bells inequality have been made since 1970s, but in these experiments significant fraction of generated photons is lost during the measurement process and caused a detection loophole, the detection loophole points out that even maximally entangled particles when measured with low-quantum-efficiency detectors can be explained by a local realistic theory. Increasing the generation and detection efficiencies of multimode SPDC photons has been a difficult technical challenge for decades and only recently higher heralding efficiencies, up to $83 \%$ have been achieved and so loophole-free tests of Bell's inequality have been conducted [14], [36], [37]. .

Furthermore, single photons need to be generated in well-defined spatial and temporal modes to be appropriate for applications in quantum information processing. 
Many quantum information protocols, such as quantum repeaters [19] and deviceindependent quantum key distribution [17] are based on single-photon sources producing in pure and indistinguishable states, with ideally zero second-order autocorrelation $g^{(2)}(0)$ which means negligible emission of photons in higher numbers. Single-photon sources also need to be narrowband to be compatible with the solid state quantum memories and MHZ bandwidth of atomic absorption lines.

Highly efficient photon-number-resolving (PNR) detectors are the essential requirement to achieve high heralding ratios for generating single-photons with low $g^{(2)}(0)$, it also allowed us to herald on photons with higher photon numbers.

In this chapter, I present the implementation of cavity-enhanced SPDC for highfidelity photon pair generation in an intrinsically stable OPO, whose well-defined cavity modes were used to herald photons in narrowband $(\sim 10 \mathrm{MHz})$ and $T E M_{00}$ quantum states, with up to $80 \%$ efficiency in preliminary results.

The heralding and measurements were performed by photon-number-resolving, high-quantum-efficiency, transition-edge-sensors (TES) built at NIST by Sae Woo Nam's group [38].

This chapter is organized as follows. First, I introduce the TES and discuss the data analysis technique we employed to study photon-number-resolved data. Then in section 2, I explain our efforts towards efficient generation and heralding of singlephotons using single-pass spontaneous parametric down conversion. In section 3 , I discuss implementation of an optical cavity and how it improved the quality of the 
single-photon source. I also study the optical cavity behavior in different operation regimes, and observe the onset of stimulated emission in the optical cavity operating well below the oscillation threshold.

\subsection{Photon-number-resolving transition-edge-sensors}

A TES is a PNR detector that exploits the strongly temperature-dependent resistance of the superconducting phase transition, see Fig. 2.2. It efficiently (>90\%) absorbs the thermal energy of the photon and converts it to electronic signal which is proportional to the energy of the absorbed photon [38. The TES operates near T=100 $\mathrm{mK}$, which is at the onset of superconductivity in tungsten, the TES active device material. The TES temperature is carefully tuned to sit at the bottom of the superconducting transition edge, where a small temperature increase due photon absorption will result in a big change in resistivity, Fig. 2.2, which will be further picked up and amplified by SQUID (Superconducting Quantum Interferometric Device) electronics.

The electronic signal generated by the TES from the monochromatic continuouswave $(\mathrm{CW})$ light at $1064 \mathrm{~nm}$ light is shown in Fig. 2.3.a. Photons arrive at the detector at random times and in different numbers, which gives rise to different peak heights. We zoomed in on photon peaks in Fig. 2.3. b, which shows that each peak consists in a sharp rising edge, of the order of few hundred nanoseconds, followed by a cooling decay of few $\mu s$, during which the detector is not "dead" and can still detect new photons. We have decreased the signal level to one peak per $\mu s$ to avoid photon 


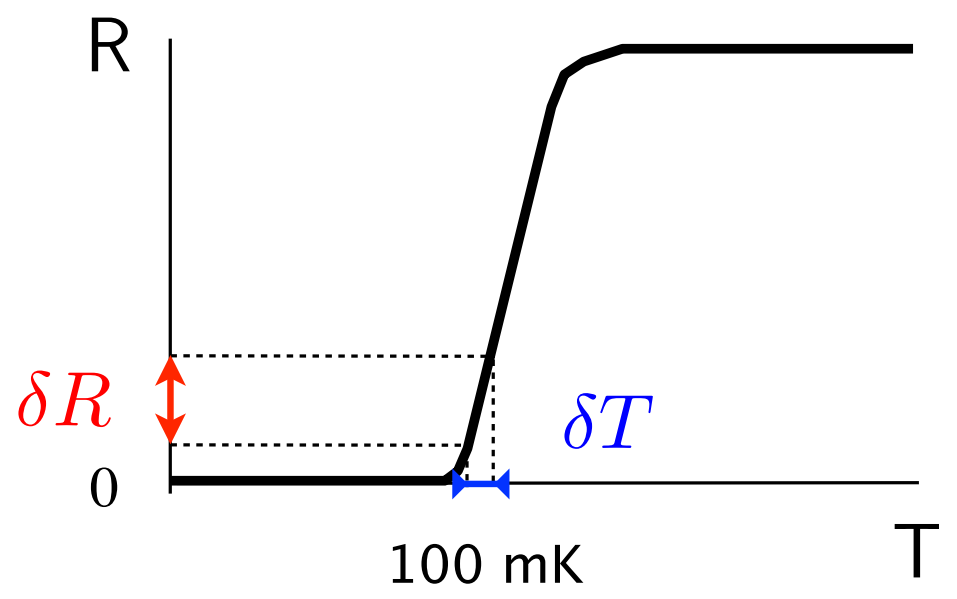

Figure 2.2: Resistivity vs temperature for the tungsten film. TES operates at the superconducting transition edge temperature, near $100 \mathrm{mK}$. The superconducting transition edge connects the superconducting regime on the left to the normal regime on the right.

pileups, where a second photon arrives while the first photon is still on the cooling decay part of the signal. Photon peaks have different peak heights which indicates different photon numbers.

There is residual uncertainty in the peak heights due to the readout noise of the detector electronics Fig. 2.3.a. For accurate photon counting statistics a photon arrival time should be identified and its height must be quantized.Each detection event is identified by finding the rising edges in the signal and is characterized as a photon event if it rises above a predefined threshold, which is set manually during the calibration process and is designed to be above the average noise level. The first few points of each photon event are recorded and summed up as a characteristic of each 
peak that contains information about the peak heigh. We sum up only the first few points of each photon peak as photon births at the rising edge and the decaying part of the peak is irrelevant to the photon characteristics, here we summed up 3 sample points out of 10 total points associated to a photon peak and called it peak sum (PS).

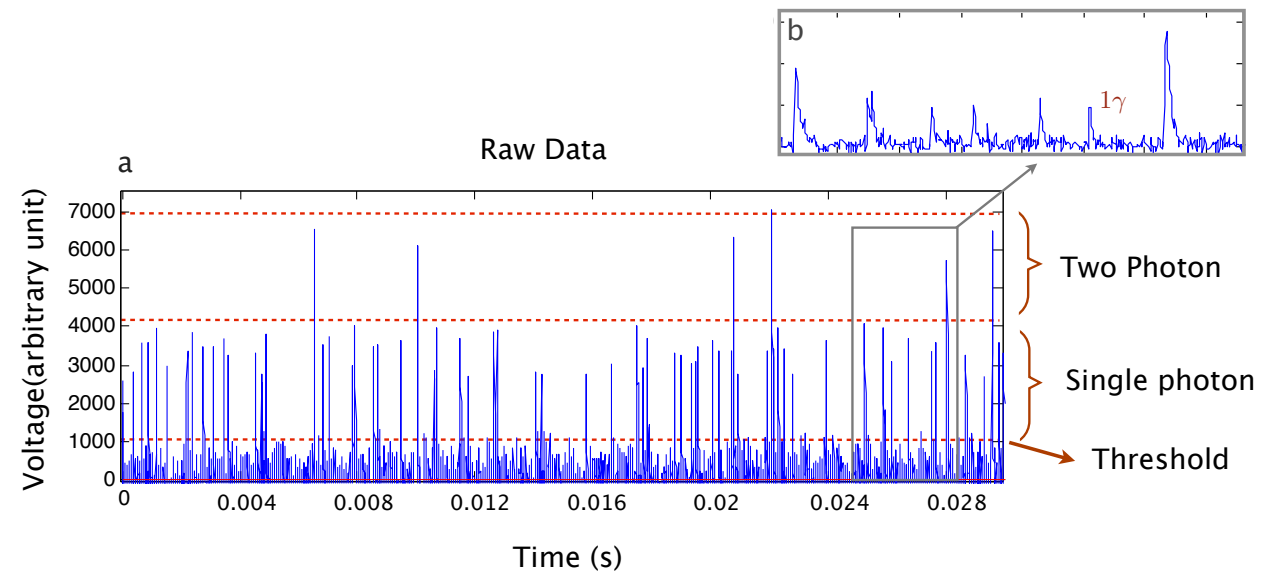

Figure 2.3: TES data. a) Raw TES data appears on the TES in the CW regime, it consists of single and two photon peaks. b) TES raw data zoomed in to show the sharp rising edge which takes hundreds of ns, and the slow falling edge of $4 \mu \mathrm{s}$.

We then build a histogram of these peak sums as presented in Fig. 2.4. The histogram has a continuous spread of numbers instead of well separated delta functions for 1,2 and 3 photons, as was expected from the small uncertainties on peak heights. However, three Gaussian-like peaks in the histogram are very well resolved and each of them corresponds to a photon number.

One can assign a boundary between peaks, and based on these boundaries, one can define quantization thresholds as in the Fig. 2.4. Quantized photon heights are presented in Fig. 2.5. 


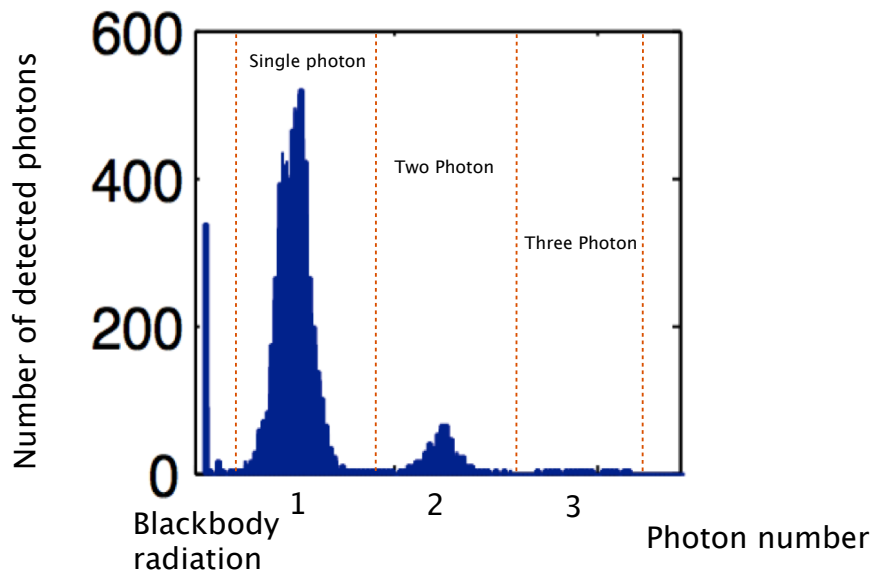

Figure 2.4: A histogram of peak sums used to decide the quantization thresholds.

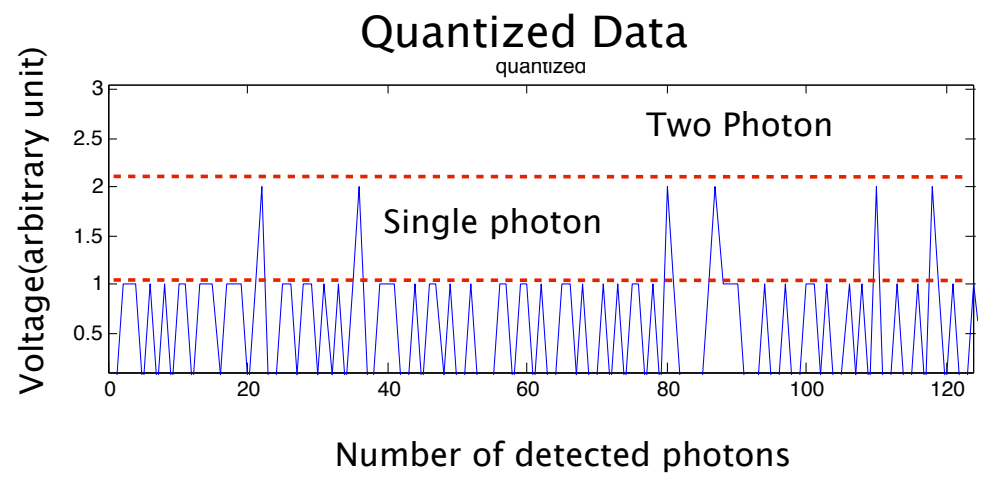

Figure 2.5: Quantized TES data. 
Implementing PNR detectors allowed us to study the photon number statistics of classical and quantum states, it also enabled us to directly calculate the quantum degree of second-order coherence, $g^{2}(0)$, which is a good measure of the quality of the heralded single photon source. The new electronic board designed at NIST enabled us to simultaneously operate 5 TES detectors.

\subsection{Heralded single photon source}

In this section, I focus on single-photon generation based on the spontaneous parametric down conversion (SPDC) in $\chi^{(2)}$ crystals. I explain our efforts towards increasing the heralding ratio and fidelity of the single-photon source before implementing the optical cavity.

We used both periodically poled $\mathrm{KTiOPO}_{4}(\mathrm{PPKTP})$ and hydrothermally grown KTP (HGKTP) crystals to spontaneously down convert 532nm pump photons into a pair of $1064 \mathrm{~nm}$, infrared (IR) photons in:

$$
|\psi\rangle=\frac{1}{\cosh r} \sum_{n=0}^{\infty} \tanh ^{n} r|n\rangle_{\text {signal }}|n\rangle_{\text {idler }}
$$

Signal and idler fields are therefore always generated in pairs, hence with correlated photon numbers.

However, the SPDC emission is multimode in nature.In the low pump power regime, where $r$ is small, photons are mostly emitted in photon pairs and the total emitted state is described by 


$$
\left|\psi_{S P D C}\right\rangle_{n}=\int d \omega_{s, i} d \vec{k}_{s} d \vec{k}_{i} \psi\left(\vec{k}_{s}, \omega_{s}, \vec{k}_{i}, \omega_{i}\right) a_{\omega_{s}, \vec{k}_{s}}^{\dagger} a_{\omega_{i}, \vec{k}_{i}}^{\dagger}|v a c\rangle
$$

where $\omega_{s}, \omega_{i}$ and $\overrightarrow{k_{s}}, \overrightarrow{k_{i}}$ are respectively the frequency and the wave vector of the signal and idler photons. $\omega_{s, i}$ and $k_{s, i}$ can carry different values, however they are constrained to obey the energy conservation:

$$
\omega_{i}+\omega_{s}=\omega_{p}
$$

and the phase matching condition:

$$
\overrightarrow{k_{i}}+\overrightarrow{k_{s}}=\overrightarrow{k_{p}}
$$

\subsubsection{Experimental setup}

We implemented a type-two SPDC process, in which photons are generated in orthogonal polarizations and can be spatially separated using a polarizing beam splitter (PBS). We then sent one polarization directly to the PNR detector. Measuring a single photon (n photons) on this detector should herald the presence of a single-photon (n photons) in the heralded arm. The experimental setup is shown in the Fig. 2.6.

The nonlinear crystal is pumped with an Innolight Diabolo laser. This laser delivers $100 \mathrm{~mW}$ of infrared light at $1064 \mathrm{~nm}$ and $600 \mathrm{~mW}$ of green light at 532 nm. These light beams were both sent to the crystal, the green light was used as the pump for the SPDC process and the infrared (IR) one, as a seed beam for alignment purposes. Note that part of the seed beam is also frequency-doubled inside 


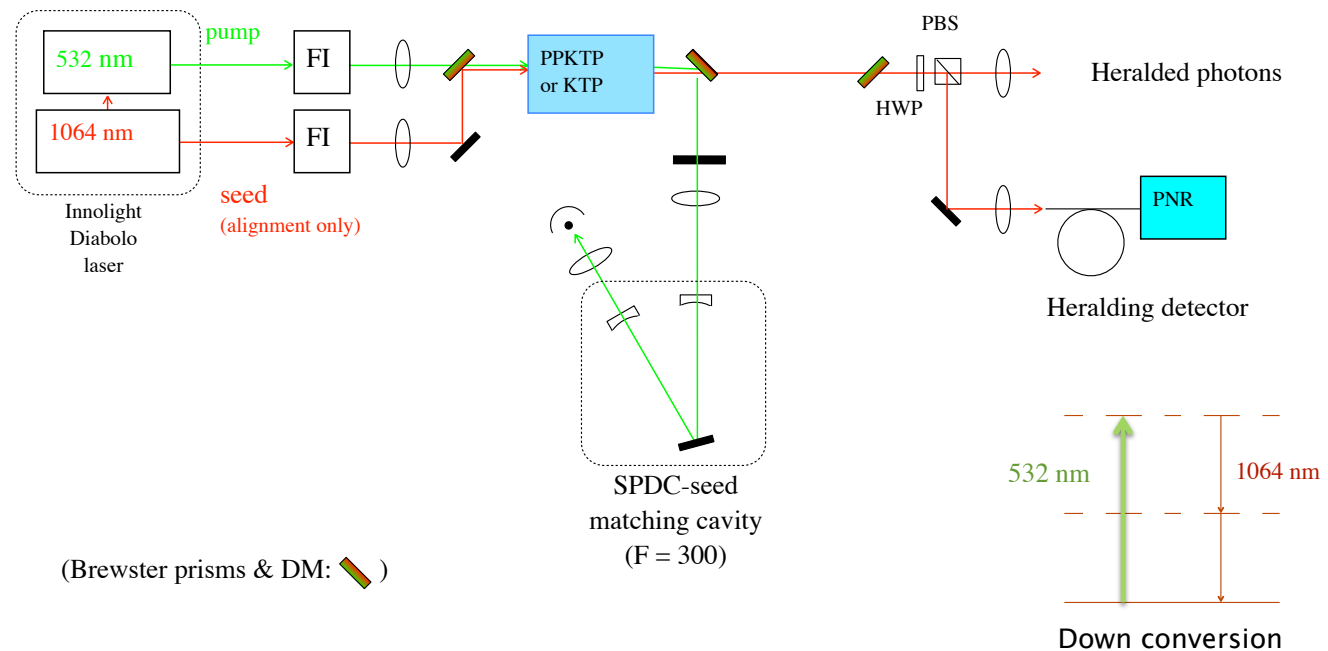

Figure 2.6: Experimental setup: A $532 \mathrm{~nm}$ pump beam is sent to a $\chi^{(2)}$ nonlinear crystal and spontaneously down converts to orthogonally polarized signal and idler fields. Which are separated by a PBS and sent to the heralding and heralded detectors.An extra Fabry-Perot cavity is implemented for alignment of, $1064 \mathrm{~nm}$, seed beam to the low-intensity SPDC modes.

the Diabolo laser to generate the green pump beam, so the frequency of the seed is exactly half of the pump frequency.

In order to overlap the seed and SPDC beams spatially, we mode matched the pump beam as well as the second harmonic of the seed, generated in the SPDC crystal, to another Fabry-Perot cavity. The cavity finesse $(F)$ was 300 and its length $30 \mathrm{~cm}$. Hence, careful alignment of these two beams guarantees the spatial overlap of the seed beam and the SPDC modes over 30 meters. We then aligned the seed to optical elements and fiber coupled TES. We achieved more than $91 \pm 3 \%$ coupling efficiency on coupling the seed beams to the $1550 \mathrm{~nm}$, SMF 28 single mode fibers.

Finally, we blocked the seed and sent SPDC modes to optical elements and fiber 
coupled detectors and consistently achieved maximal signal and coincidence rates on the heralding and heralded detectors, as scanning the TES fiber position in X and Y directions dropped the signal level on TES, Fig. 2.7. It shows the success in our alignment method.

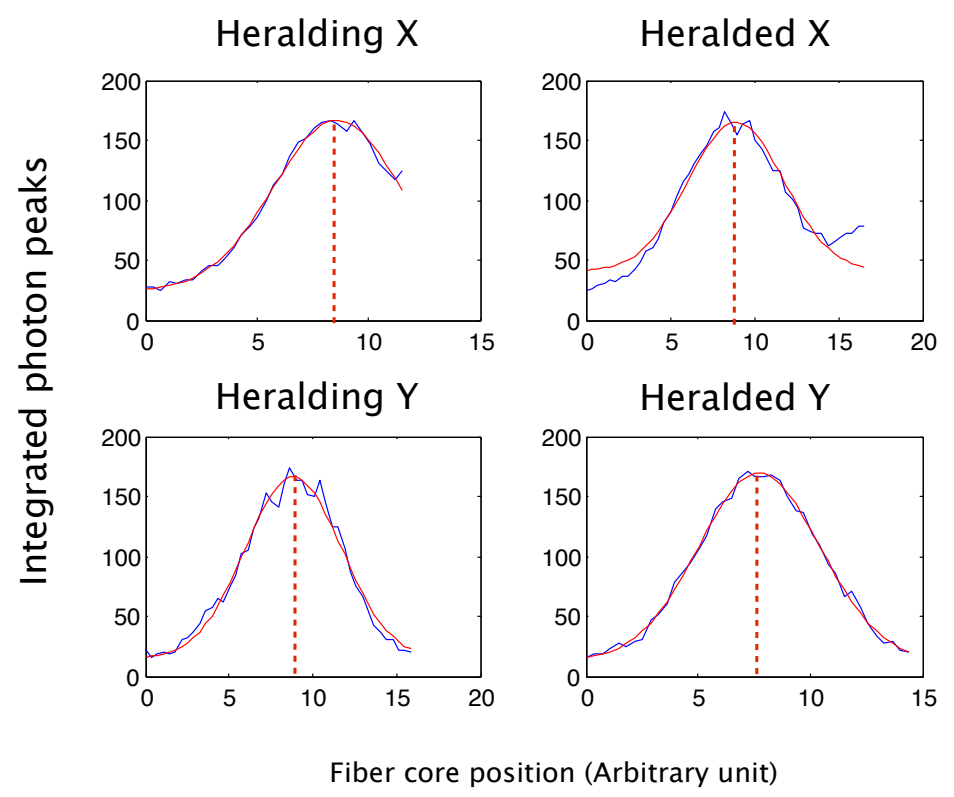

Figure 2.7: Integrated photon counts, over $13 \mathrm{~ms}$, on the heralding and heralded channels vs fiber position. The integrated peak were always at the maximum, red dashed lines, after aligning SPDC with seed.

Extensive studies were conducted on the pump optimum focusing condition on the nonlinear crystal to optimize it for various SPDC properties [39] such as, the joint spectral density; the pair collection probability; the heralding ratio (pair/single photon collection ratio), etc. Some properties can be jointly optimized while others require a trade-off. We decided to design the pump and SPDC focusing conditions on the crystal to increase the heralding ratio without sacrificing for the SPDC pair 
generation. Defining the focusing parameter as,

$$
\xi_{j}=\frac{L}{k_{j} w_{j}^{2}}
$$

then we chose our focusing condition to obey,

$$
\xi_{\text {signal }}=\xi_{\text {idler }}=\xi_{\text {pump }}=\frac{L}{k_{p} w_{p, s, i}^{2}}=0.23
$$

Pump has $w_{p}=60 \mu \mathrm{m}$ waist on the $L=1 \mathrm{~cm}$ long PPKTP crystal. We then carefully designed the optics elements behind the fiber couplers to optimize them for efficient collection of SPDC modes satisfying $w_{S P D C}=\sqrt{2} w_{\text {pump }}$, Eq. 2.7. .

Despite all careful alignments and designs, we were not able to achieve near unity correlation between signal and idler photons, as was expected from theory and nearunity detection efficiency of TES detectors. The correlation efficiency (number of coincidences/ average number of photons on each detector ) was $C=25 \% \pm 5 \%$. We placed a TES detector in each of the heralding and heralded channels to determine coincidence events and average photon numbers.

We then replaced the nonlinear crystal with an ultralow loss, hydrothermally grown KTP (HGKTP) crystal. We believed that HGKTP crystals are better candidates for single-photon generation due to their high damage resistance and low loss. However, these crystals are birefringently phase matched (BPM) [40], which causes walkoff between the orthogonally polarized signal and idler beams in the crystal. This makes alignment quite challenging. So we decided to employ two identical HGKTP crystals and place them 180 degree rotated with respect to each other in order to 
compensate for the walkoff. By careful crystal polarimetry, we oriented each crystal's X-principal axes separately and with respect to each other.

\subsubsection{Crystal polarimetry}

Born and Wolf [41] have analyzed birefringently phase matched crystals and described polarimetry methods for determining several characteristics of a crystal, such as the position of the principal axes and the refractive indices. We briefly explain their method then describe how we used it to study HGKTP crystals.

Birefringence is a property of anisotropic materials where different polarizations of light "see" different refractive indices. In addition, the poynting vector may also "walk off" the wave vector. It causes orthogonal polarizations to travel as illustrated in Fig. 2.8 .

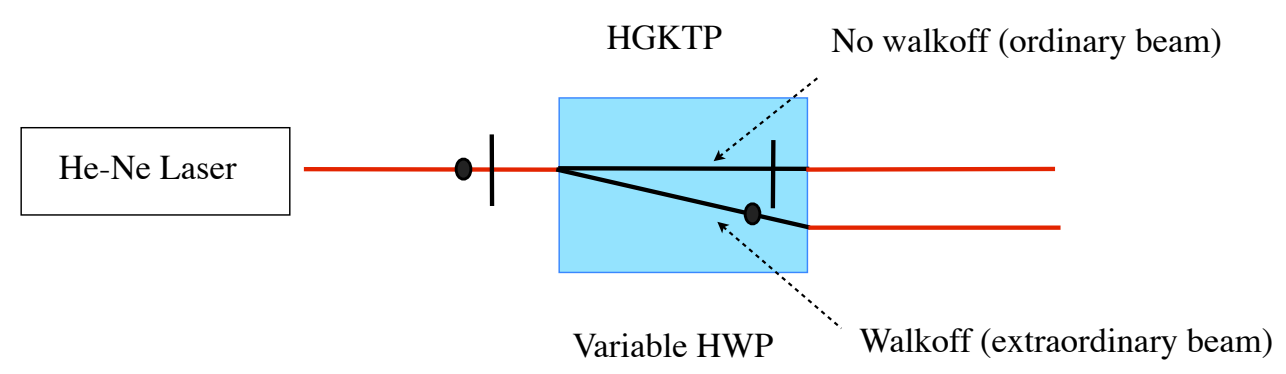

Figure 2.8: Propagation off a principal axis in a birefringent crystal can walk off the extraordinary beam [41]

The difference between two refractive indices $\Delta n$ for two orthogonally polarized beams depends on the direction of the incident beam respect to the crystal's principal 
axis, in our case the $\mathrm{x}$ axis of KTP. $\Delta n$ can be calculated as [41]:

$$
\Delta n=\left(n_{e}-n_{o}\right) \sin ^{2} \theta
$$

Where, $n_{o}$ and $n_{e}$ are the ordinary and extraordinary refractive indices and $\theta$ denotes the angle which the traveling wave normal makes with the $\mathrm{x}$ axis. The phase difference between traveling orthogonally polarized beams depends on the distance $\rho$ traveled by beams in the crystal and can be calculated as:

$$
\delta=\frac{2 \pi \rho}{\lambda}\left(n_{e}-n_{o}\right) \sin ^{2} \theta
$$

Hence the surfaces of constant phase difference are:

$$
\rho \sin ^{2} \theta=C
$$

The intersection of these constant phase planes with the plane of the crystal surface corresponds to curves with constant phase difference on the crystal surface also called "isochromates", these curves approximates to hyperbolae for the special crystal cut where principal axes make a relatively large angle with the crystal faces which is a case for the HGKTP crystals.

To observe "isochromates" curves, we need to tightly focus the beam to generate beams traveling with large angles $\theta$ in the crystal, then one also needs to place two polarizers both at $45 \mathrm{deg}$ before and after the crystal. The first polarizer generates two orthogonally polarized beams with equal amplitudes, the birefringent crystal then acts as a variable wave-plate and adds a relative phase shift $\delta$ between polarizations and 
generates an elliptically polarized beam. Despite the phase shift, the total amplitude of the beam is constant for all beams in all different directions. The second polarizer, parallel or perpendicular to the first one, analyzes the elliptical polarization created in the crystal, which also depends on the propagation direction.

So the second polarizer translates the constant phase shift curves to the constant amplitude curves and generate interference fringes Fig. 2.9. The beam passing through the principal axis of the crystal $\theta=0$, does not experience any phase shift between polarizations $\delta=0$ and so pass through the second polarizer and results in a bright (for parallel polarizers) or dark (for orthogonal polarizers) zone in the middle of the fringes.

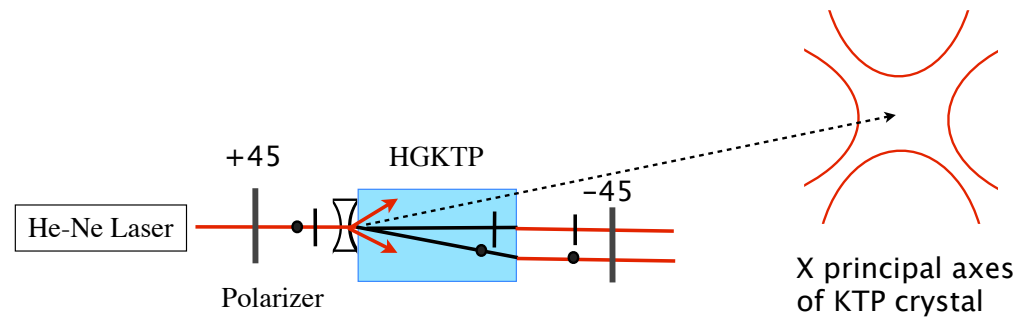

Figure 2.9: Tightly focused cross polarized beam generates an interference fringe," isochromatic" curve, upon placing two perpendicular HWPs before and after the HGKTP.

We used a HeNe laser to study HGKTP crystals. The He-Ne laser operates at 633 $\mathrm{nm}$ wavelength, which is far from the phase matching bandwidth of the KTP crystals 
in which the downconversion process is not efficient. Also, the crystal's anti reflection (AR) coating is not designed to work at $633 \mathrm{~nm}$, which will allow us to observe useful etalon fringes.

We generated crystal fringes and observed parabolic "isochromatic" curves, Fig. 2.10.a. In addition to crystal fringes, we can also see circular Fabry-Perot, or etalon, fringes located on the right of fringe Fig. 2.10,a . These etalon fringes are featured in Fig.2.10,b. Thus the bright spot in the middle of the crystal fringes Fig. 2.10.a locates the direction of the x-principal axis while the dark spot in the middle of the Fabry-Perot fringes points out the direction of the normal incidence to the crystal. Fig. 2.10.a clearly demonstrates that centers of these two fringes does not overlap. It means that the HGKTP crystal's surface is cut with an angle with respect to the x-principal axis

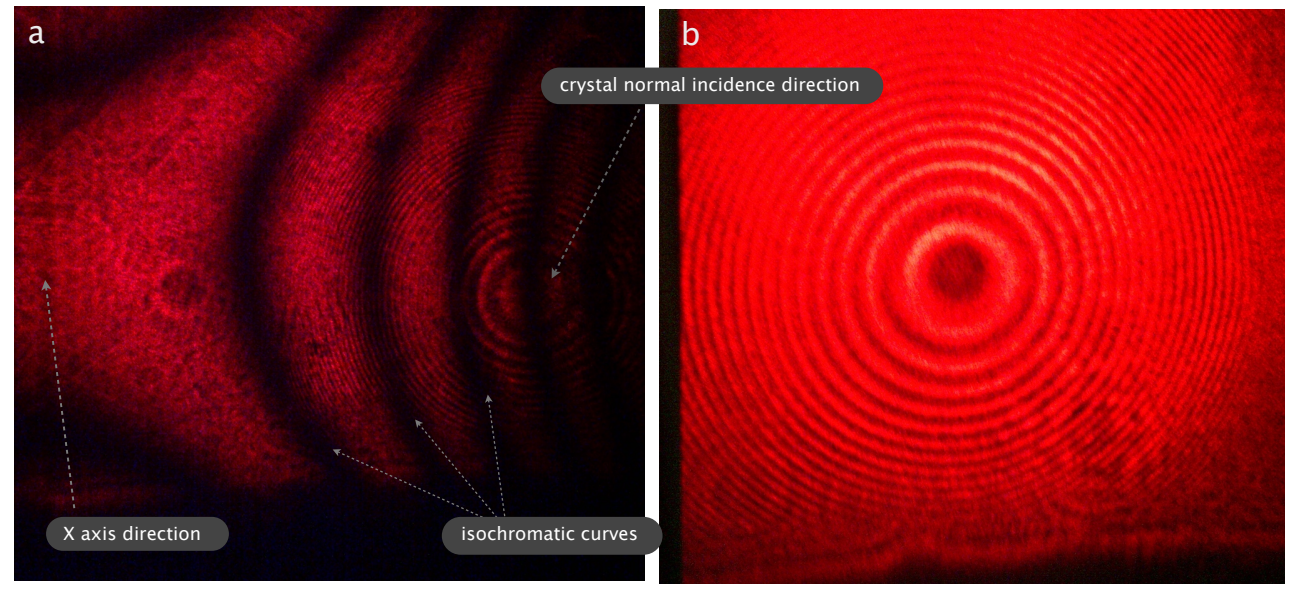

Figure 2.10: Crystal polarimetry fringes a) isochromatic curves and etalon fringes. b) etalon fringes

In fact, BPM in HGKTP crystals mandates that the $\mathrm{x}$ axis be at $22^{\circ}$ normal 
incidence, an angle is carefully designed to ensure the orthogonally polarized signal and idler beams poses appropriate refractive indices to fulfill the phase matching condition Eq. 2.5) for the frequency degenerate signal and idler photons with $\omega_{s}=$ $\omega_{i}=\frac{\omega_{p}}{2}$.

$$
n_{s}+n_{i}=2 n_{p}
$$

The angle between the crystal surface normal and the $\mathrm{x}$ axes in HGKTP crystals Fig. 2.10. a shows that beam is not traveling parallel to the $\mathrm{x}$ axis $\theta \neq 0$ and so the refractive indices for ordinary and extraordinary beam are different $\left(n_{o} \neq n_{e}\right)$, which results in walk off between orthogonally polarized signal and idler beams Fig. 2.8 .

For this reason, we employed another identical HGKTP crystal and oriented it $180^{\circ}$ respect to the first one, to compensate for the phase shift and the walk off. The second crystal completely takes care of the first crystal effects and so the ordinary and extraordinary beams leave the crystal without a relative displacement Fig. 2.11.

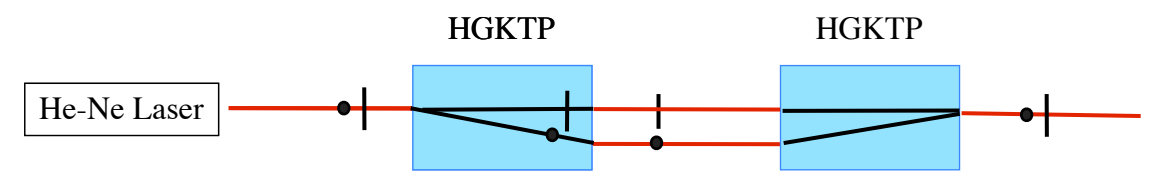

Figure 2.11: Identical HGKTP crystal placed at $180^{\circ}$ with respect to the initial HGKTP crystal and compensated for the walkoff and phase shift effects.

Crystal and Fabry-Perot fringes for two identical HGKTP crystals placed $180^{\circ}$ with respect to each other are shown in Fig. 2.12. The resulting interference fringes overlap which clearly shows that the effective $\mathrm{x}$ axis of the combined crystals is parallel 
to the normal incidence Fig. 2.12.

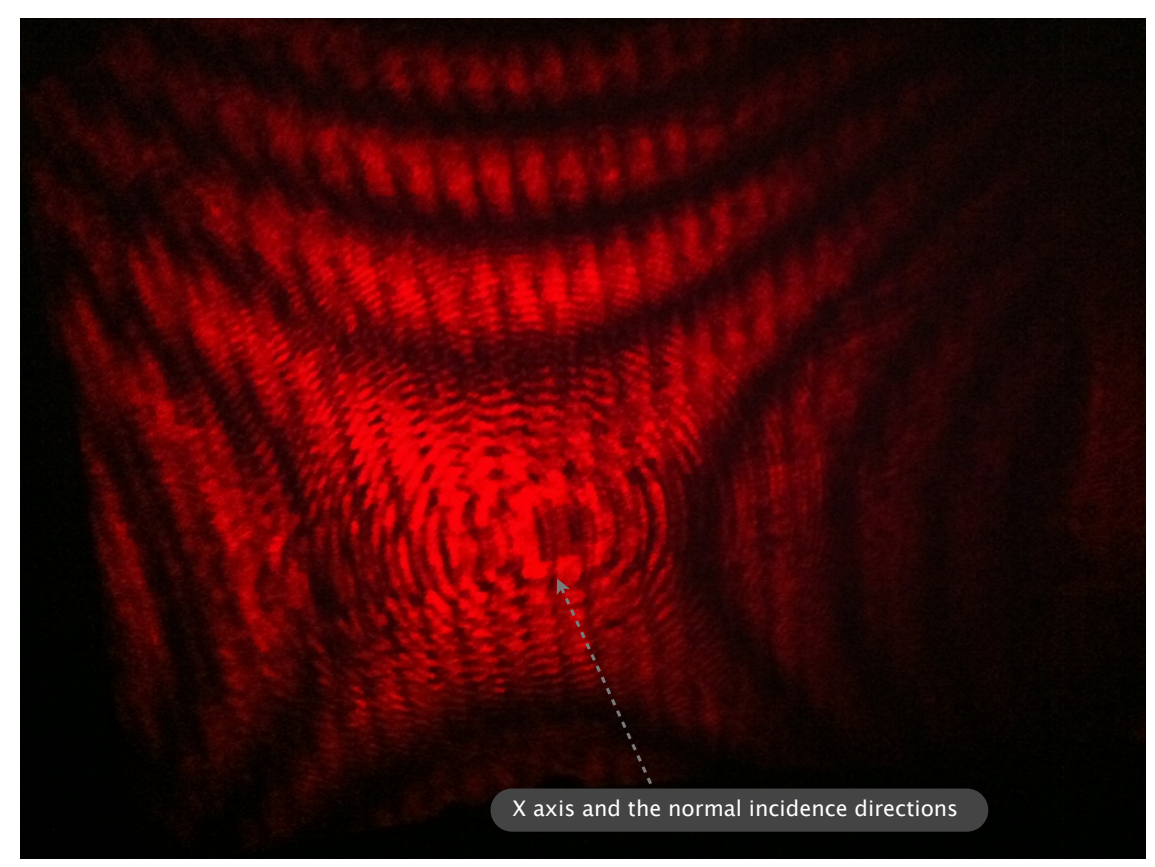

Figure 2.12: Isochromatic curves and etalon fringes, after compensating for the walk off.

Therefore, using the He-Ne laser and the crystal polarimetry allowed us to successfully compensate for the walk off and guarantees the spatial overlap between signal and idler after the crystals.

However, the maximum achievable correlation efficiency using compensated HGKTP crystals is $C=15 \pm 5 \%$ which is even lower than the correlation in the PPKTP crystals with $C=25 \pm 5 \%$.

We later realized that is probably be due to the fact that SPDC photons are generated randomly at different positions in the crystals, and this method can only compensate the walk off for photons generated at the very beginning of the first 
crystal and will only partially compensate for the rest.

So far, we have tried both of the PPKTP and HGKP crystals to spontaneously generate correlated signal and idler photons, however despite all careful alignment and crystal polarimetry we have not been able to observe near-unity photon number correlation.

At this point, we concluded that the reason for low photon number correlation is the multimode nature of SPDC modes. In fact, the phasematching conditions, Eq. (2.5) ,allows the generation of photons with various frequencies and in different directions Fig. 2.13 .

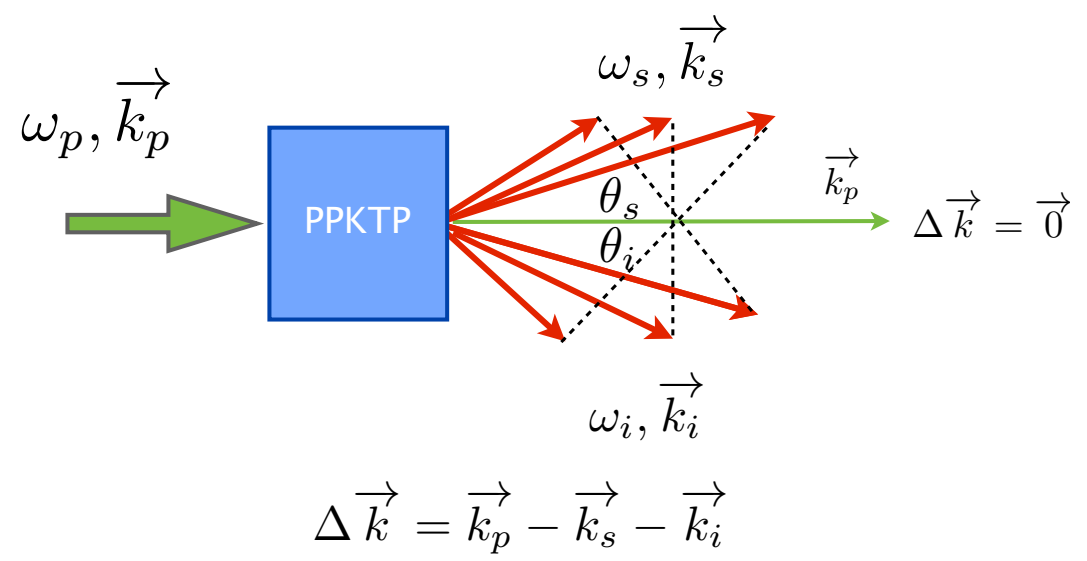

Figure 2.13: SPDC process, phase matching condition $\Delta k=0$ allows the creation of signal and idler photons in different directions.

Therefore, even at $\Delta \vec{k}=\overrightarrow{0}$, signal and idler modes can be emitted in various directions or equivalently in higher order TEM modes, so both the signal and idler modes are not simultaneously compatible with the single mode of an optical fiber and efficient collection of the multimode SPDC pairs on the fiber coupled TES detectors 
is nearly impossible, as TES detectors are coupled to the $1550 \mathrm{~nm}$ single-mode fiber.

What's even worse, the phase matching bandwidth also allows for a vector phase mismatch $\Delta \vec{k} \neq \overrightarrow{0}$, in this case the situation is more critical and SPDC can create completely decorrelated photons as in Fig. 2.14. In this case one mode, say the idler, can efficiently couple to the fiber but other one, the signal, is completely off and will be missed on the detector.

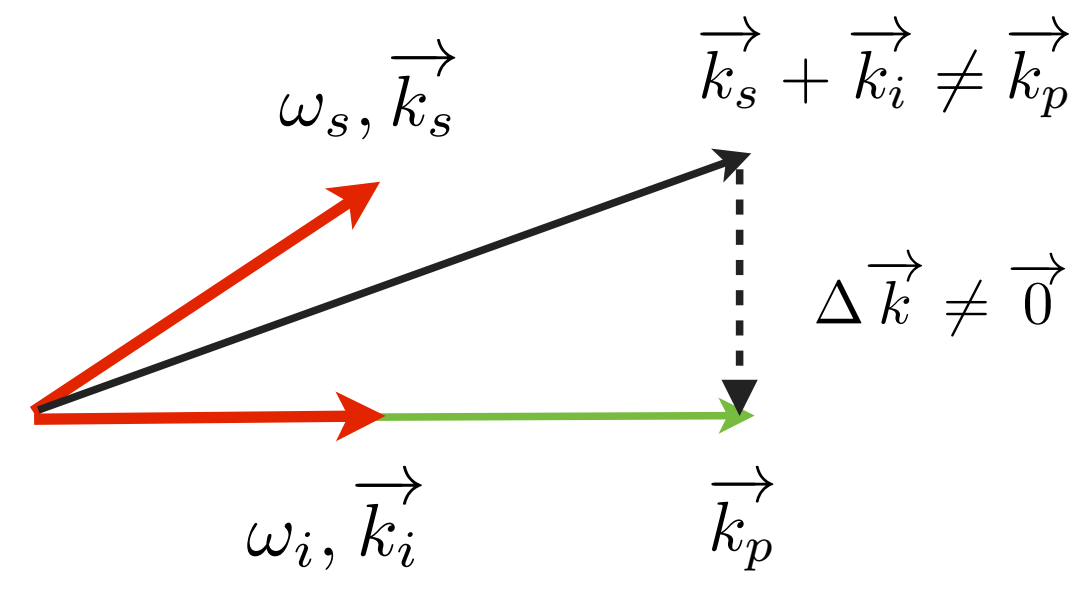

Figure 2.14: Phase mismatched SPDC emission.

Thus, we decided to clean up the multimode and broadband SPDC modes, by use of a 1 GHZ-bandwidth filtering cavity, with as large a free spectral range as possible, in addition to a interference filter, to filter all SPDC modes and only pass the spectrally and spatially well defined signal and idlers.

\subsubsection{Filtering Cavity}

We inserted a $0.5 \mathrm{~mm}$ long, 300 finesse filtering cavity in the SPDC path. The cavity was carefully designed to have relatively small bandwidth of 1 GHZ along 
with large free spectral range (FSR) of $300 \mathrm{GHZ}$ to efficiently filter the broadband SPDC spectrum with $\sim 1 \mathrm{THz}$ bandwidth. The filtering cavity is a Fabry-Prot cavity, consisting of two opposing spherical mirrors with $\mathrm{f}=5 \mathrm{~cm}$, with small mirror separation distance of $\mathrm{L}=0.5 \mathrm{~mm}$. Signal and idler photons are generated anywhere within the

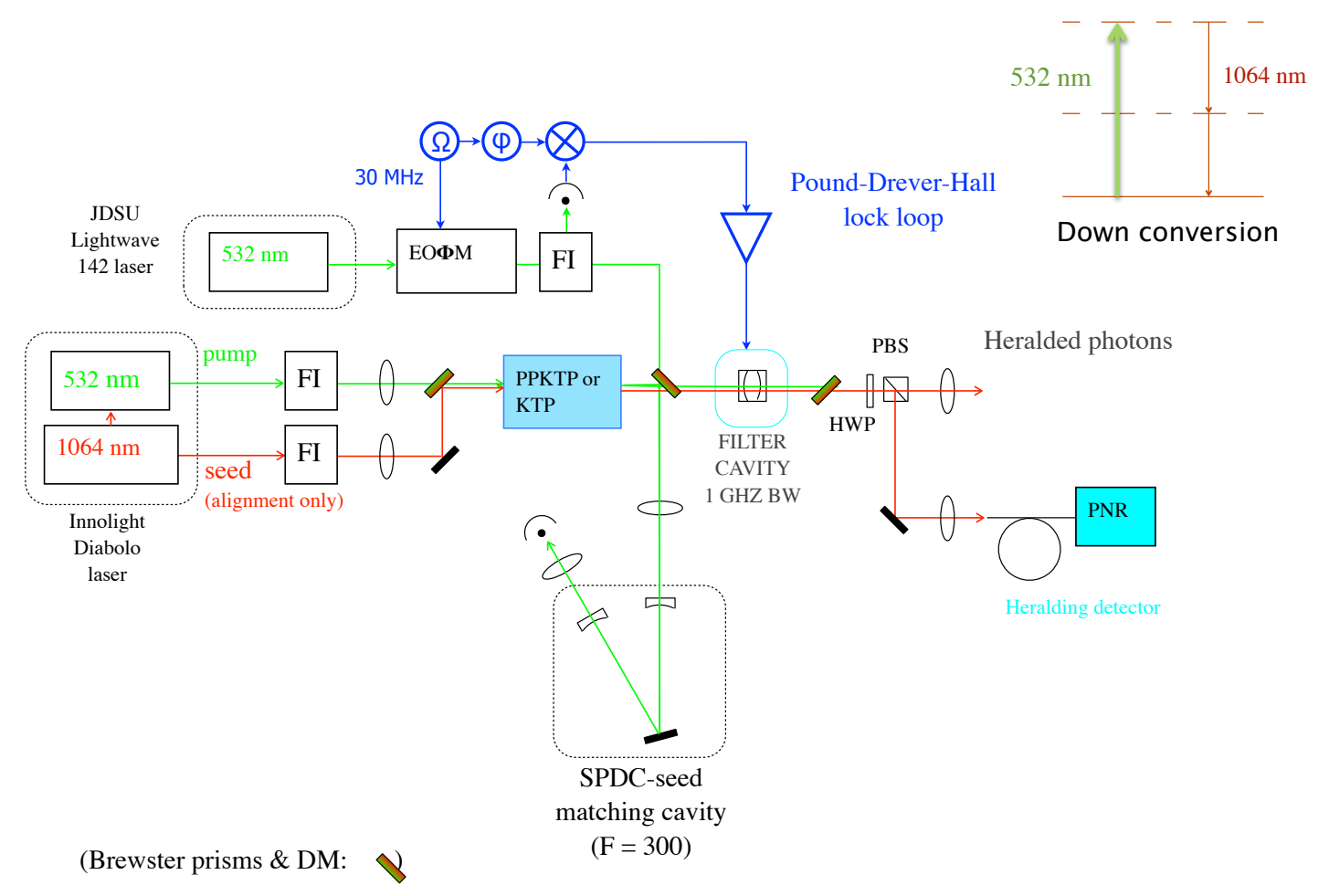

Figure 2.15: The filtering cavity is placed on the signal and idler paths to filter out a spatially and spectrally well-defined mode. Another $532 \mathrm{~nm}$ laser is employed to produce locking signal for locking the filtering cavity.

SPDC frequency spectrum, see Fig. 2.16, the only constraint being that they should satisfy $\omega_{\text {signal }}+\omega_{\text {idler }}=\omega_{\text {pump }}$ imposed by the energy conservation Eq. (2.4). Thus, 
signal and idler frequencies can be written as:

$$
\begin{aligned}
\omega_{\text {signal }} & =\frac{\omega_{p}}{2} \pm \Delta \omega \\
\omega_{\text {idler }} & =\frac{\omega_{p}}{2} \mp \Delta \omega
\end{aligned}
$$

Selecting a small fraction of the spectrum by inserting the filtering cavity resonant at frequencies far from the the SPDC central frequency where $\left(\omega_{s}=\omega_{i}=\frac{\omega_{p}}{2}\right)$, results in passing only one of the signal or idler photons through the cavity but filtering the other and so should cause zero photon number correlation (Fig. 2.16.a). On the other hand, at near degenerate frequencies, both signal and idler photons have frequencies within the filtering cavity BW and both pass through the cavity.
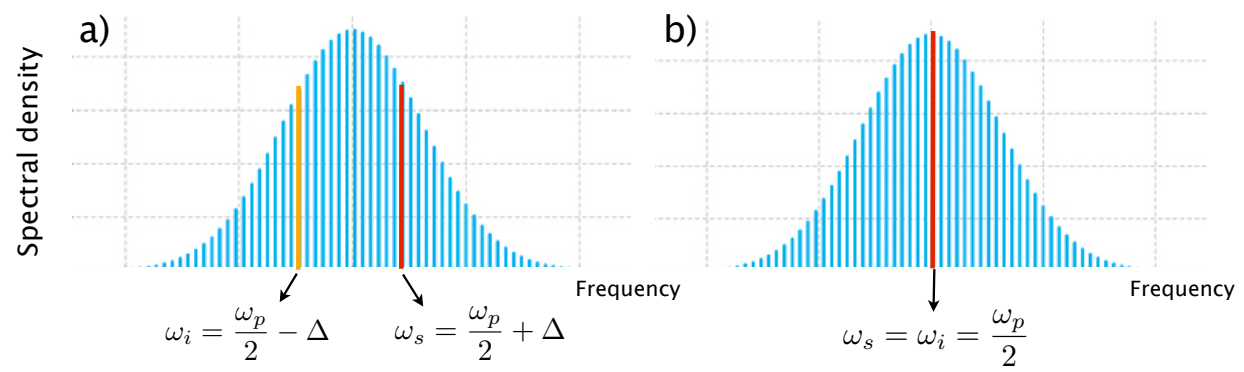

Figure 2.16: SPDC frequency spectrum for signal and idler a) the filtering cavity is locked far from the SPDC central frequency, so the signal passes (red) but idler is blocked (yellow) by the filter. b) the filtering cavity is locked exactly at half of the pump frequency and both the signal and idler are passing through the cavity

Accordingly, careful locking of the filtering cavity on the central SPDC frequency $\frac{\omega_{p}}{2}$ is really essential to achieve maximum photon-number correlation. We used another green laser to generate locking signal for locking the filtering cavity precisely at the desire length with the Pound-Drever-Hall technique. The seed beam is imple- 
mented as the frequency reference as it has exactly half of the pump frequency and the locking laser frequency is properly tuned to enable same resonance condition with the seed on the cavity. Fig. 2.17 shows the resonant modes of locking and seed beams both on the filtering cavity, their resonances are on top of each other which means they both happen exactly at the same length of the cavity and so locking the filtering cavity on the green laser resonance guarantees passing the seed beam and so the central frequency of the SPDC .

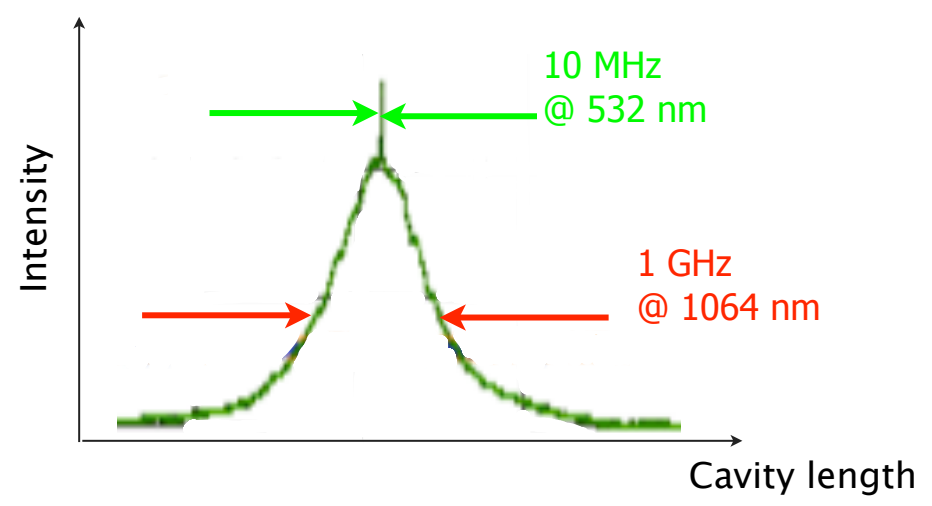

Figure 2.17: Cavity spectrum for seed at $1064 \mathrm{~nm}$ and locking laser at 532nm

The locking mode bandwidth (BW) is 100 times narrower than the seed mode $\mathrm{BW}$, therefore locking the cavity even on the edges of the locking mode spectrum will results in filtering cavity locked exactly on the maximum of the seed resonance, and therefore on degenerate part of SPDC modes. In Fig. 2.18 we show the dependence of the cavity detuning on the signal and idler correlation, we see the correlation will drop from maximum to zero by detuning and locking the filtering cavity far from the 
degenerate mode.

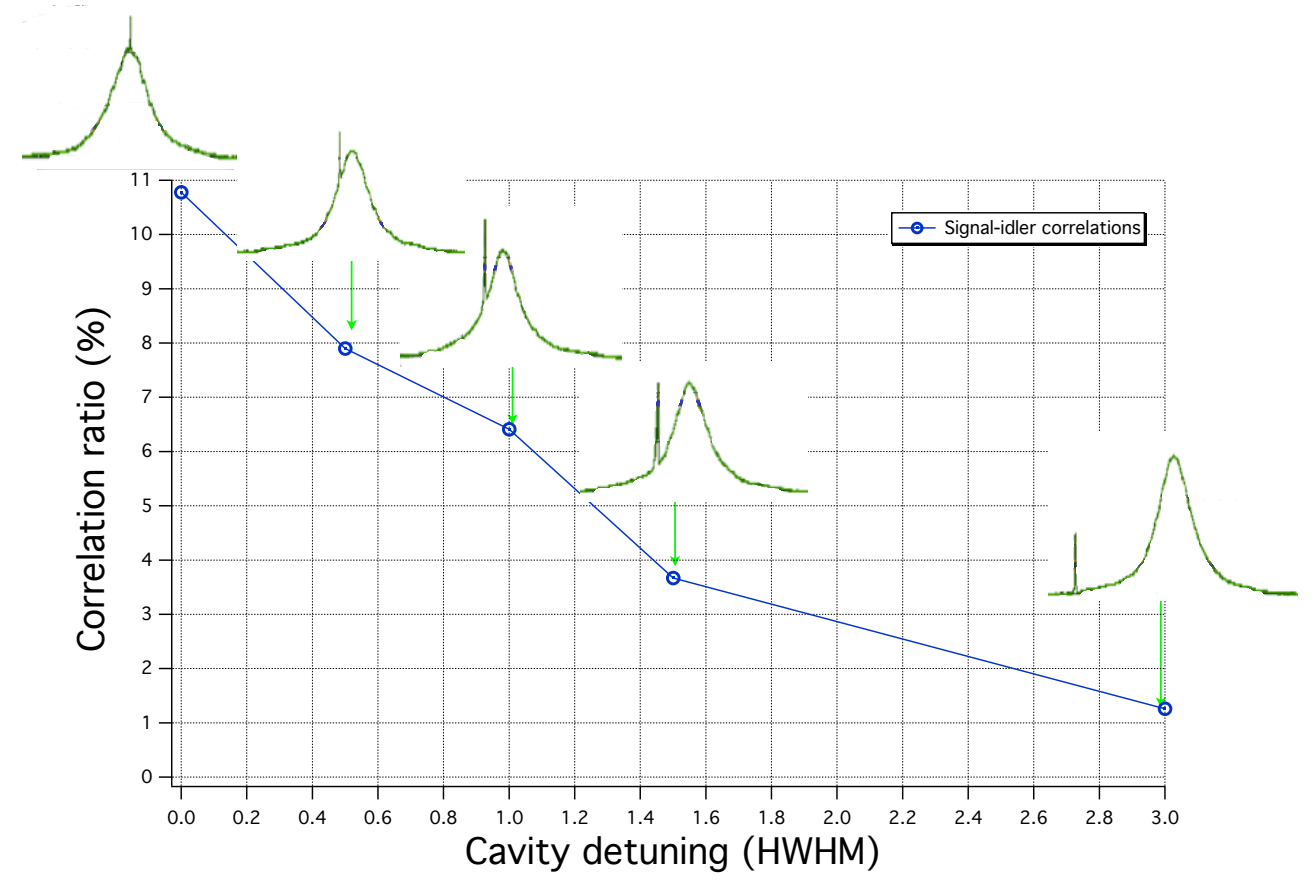

Figure 2.18: Correlation depending on the cavity detuning, it drops from maximum value to zero by detuning the cavity from degeneracy.

So we could lock the filtering cavity exactly on the degenerate frequency and achieved the optimum correlation respect to the cavity detuning from resonance.

However, the optimum correlation for HGKTP crystal after adding the cavity is still at the same level as the correlation before inserting the filtering cavity $c=10 \pm 5 \%$. In fact, inserting the filtering cavity adds extra loss which is in the order of the improvement in correlation, the loss could be due to the imperfect mode matching of the signal and idler to the cavity, or losses on mirrors, in fact the cavity throughput for the seed is only $80 \%$. There are also other sources of losses which will be discussed in detail later in the next section. 


\subsection{Cavity enhanced and high-heralding-ratio single- photon source}

We tried inserting a narrow-band $1 \mathrm{GHz}$ passive filtering cavity on the SPDC beam path in an effort to clean for the spatially and temporally well defined SPDC modes and also to improve the photon number correlation between signal and idler modes.

In fact, implementing a passive filtering cavity is the most common method executed so far for decreasing the SPDC spectrum bandwidth. However, it has serious drawbacks. Firstly, the spontaneous nature of the process makes the conversion probability directly proportional the SPDC bandwidth and simply filtering the spectrum for smaller bandwidth and longer correlation time will dramatically reduce the brightness of the signal. More importantly, the filtering cavity adds extra loss to the process due to the Lorenrtzian cavity decay which will be explained in detail later in section 2.4.1. The Lorentzian loss is independent of losses originated from imperfect mode matching of the signal and idler to the cavity.

Further, the filtering cavity cannot perfectly filter spatially well defined cavity modes as the cavity is injected with multimode and broadband SPDC modes, in which the resonance of higher order modes with off degenerate frequencies is still possible, so higher order SPDC modes can penetrate through the filtering cavity.

More importantly, the completely nonresonant modes, although very weakly transmitted by the filter cavity, are still in such large number that they can still overwhelm the "clean" doubly resonant modes. Therefore, implementing a filtering cavity after 
the down converting crystal cannot efficiently filter spatially and temporally well defined SPDC modes as was indirectly proved by observing low correlation efficiency after adding the filtering cavity.

Therefore, we decided to place the down-converting crystal in an optical cavity, thereby making an optical parametric oscillator (OPO), only well below threshold that resonates both the signal and idler modes as was first proposed and implemented by Ou's group [42]. Resonance property of cavity modes reduced the bandwidth of SPDC to that of the cavity, without adding extra loss and sacrificing source brightness.

In the free space SPDC process, pump photons downconvert into a superposition of very many different free-space field modes Eq. (2.3). However, when the down converting crystal is placed inside an optical cavity the pump photons are more likely to annihilate into spatially well-defined resonant cavity modes, which can then be efficiently collected into single-mode optical fibers for detection and communication purposes.

In this section, I initially study the input-output theory to clarify the effect of the filtering cavity and the cavity enhancement on SPDC modes, then I describe the experimental observation of cavity enhanced SPDC modes by placing PPKTP crystals inside an intrinsically stable optical cavity. Finally, I discuss about the effect of cavity enhancement for improving the heralding efficiency in single-photon generation. 


\subsubsection{Input-output formulation for optical cavities}

The input-output theory of optical cavities studies the interaction of a single cavity mode with external fields, it is a Heisenberg equation of motion for linearized quantum fields coupled with appropriate cavity boundary conditions and describes the evolution of the input field, cavity field, and output field [43].

An optical cavity is commonly described by a Hamiltonian of the form:

$$
H_{t}=H_{\text {Sys }}+H_{B a t h}+H_{I n t}
$$

Where $H_{\text {Sys }}$ is the Hamiltonian for the intracavity field mode $a, H_{\text {Bath }}$ is the free Hamiltonian for the external field modes $a_{\text {in }}, a_{\text {out }}, b_{\text {in }}, b_{\text {out }}$ and $H_{\text {int }}$ is the interaction Hamiltonian that describes the interaction between the bath and cavity fields.

For the two-sided cavity with two partially transparent mirrors with associated loss transmission coefficients $\gamma_{1}$ and $\gamma_{2}$, a single cavity mode interacts with external fields $a_{\text {in }}, a_{\text {out }}$ and $b_{\text {in }}, b_{\text {out }}$ Fig. 2.19.

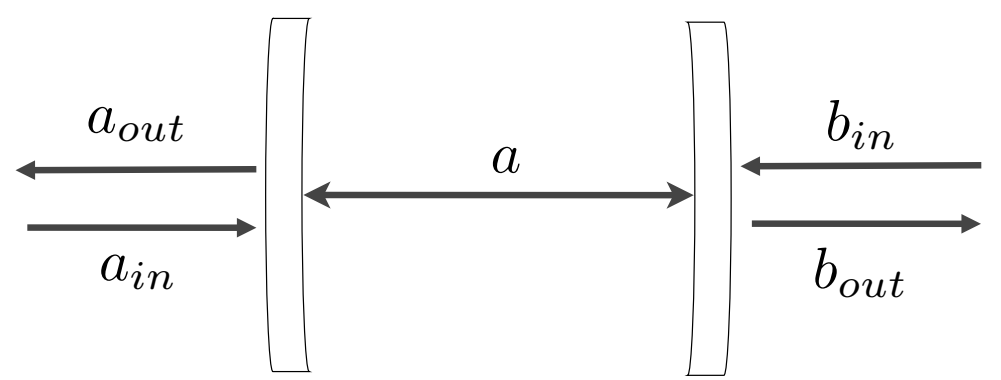

Figure 2.19: A schematic representation of the cavity field and the input and output fields for a double-sided cavity

The quantum Langevin equation for the internal field is then given by [44, 


$$
\frac{d a(t)}{d t}=-\frac{i}{\hbar}\left[a(t), H_{\text {Sys }}\right]-\frac{1}{2}\left(\gamma_{1}+\gamma_{2}\right) a(t)+\sqrt{\gamma_{1}} a_{i n}(t)+\sqrt{\gamma_{2}} b_{i n}(t)
$$

When the optical cavity is empty, for example in the passive filtering cavity case, the $H_{\text {Sys }}$ is the free Hamiltonian for the intra cavity mode $a$ :

$$
H_{S y s}=\hbar \omega_{0} a^{\dagger} a
$$

where $\omega_{0}$ is the cavity resonance frequency. For the active OPO filter a downconverting crystal is placed inside the cavity and cavity is operating well below the oscillation threshold. The pump mode of the OPO can be treated classically, then the system hamiltonian $H_{S Y S}$ is:

$$
H_{\text {Sys }}=\hbar \omega_{0} a^{\dagger} a+\frac{i \hbar}{2}\left(\epsilon a^{\dagger 2}-\epsilon^{*} a^{2}\right)
$$

where $\epsilon=\epsilon_{p} \chi$ and $\epsilon_{p}$ is the amplitude of the pump, and $\chi$ is proportional to the nonlinear susceptibility of the nonlinear medium. Therefore, Eq. (2.15) can be written as:

$$
\frac{d a(t)}{d t}=-i \omega_{0} a(t)+\epsilon a^{\dagger}-\frac{1}{2}\left(\gamma_{1}+\gamma_{2}\right) a(t)+\sqrt{\gamma_{1}} a_{i n}(t)+\sqrt{\gamma_{2}} b_{i n}(t)
$$

This is the equation of motion for the active filter but merges to the equation for the passive filtering cavity in the limit of zero nonlinear interaction $\epsilon \rightarrow 0$. 
Defining the Fourier transform of intracavity field $a$ as:

$$
a(t)=\frac{1}{\sqrt{2 \pi}} \int_{-\infty}^{\infty} e^{-i \omega(t)} a(\omega) d \omega
$$

then equations of motion, Eq. 2.18) can be written in frequency space as:

$$
-i a(\omega)=-i \omega_{0} a(\omega)+\epsilon a^{\dagger}(-\omega)-\frac{1}{2}\left(\gamma_{1}+\gamma_{2}\right) a(\omega)+\sqrt{\gamma_{1}} a_{i n}(\omega)+\sqrt{\gamma_{2}} b_{i n}(\omega)
$$

The relationship between input and output modes may be found using boundary conditions at each mirror:

$$
\begin{aligned}
& a_{\text {out }}(t)+a_{\text {in }}(t)=\sqrt{\gamma_{1}} a(t) \\
& b_{\text {out }}(t)+b_{\text {in }}(t)=\sqrt{\gamma_{2}} a(t)
\end{aligned}
$$

We use boundary conditions to eliminate the intra cavity mode $a$ in Eq. 2.20) and obtain output cavity modes $a_{\text {out }}$ and $b_{\text {out }}$ in terms of input modes $a_{\text {in }}$ and $b_{\text {in }}$, we also transform to rotating frame with $a \rightarrow e^{i \omega_{0}} a$, the equations of motion then become:

$$
\begin{aligned}
a_{\text {out }}\left(\omega_{0}+\omega\right) & =G_{1}(\omega) a_{i n}\left(\omega_{0}+\omega\right)+g_{1}(\omega) a_{i n}^{\dagger}\left(\omega_{0}-\omega\right) \\
& +G_{2}(\omega) b_{i n}\left(\omega_{0}+\omega\right)+g_{2}(\omega) b_{i n}^{\dagger}\left(\omega_{0}-\omega\right)
\end{aligned}
$$

where:

$$
\begin{array}{ll}
G_{1}(\omega)=\frac{\gamma_{1}-\gamma_{2}+2 i \omega}{\gamma_{1}+\gamma_{2}-2 i \omega}, & g_{1}(\omega)=\frac{4 \epsilon \gamma_{1}}{\left(\gamma_{1}+\gamma_{2}-2 i \omega\right)^{2}} \\
G_{2}(\omega)=\frac{2 \sqrt{\gamma_{1} \gamma_{2}}}{\gamma_{1}+\gamma_{2}-2 i \omega}, & g_{2}(\omega)=\frac{4 \epsilon \sqrt{\gamma_{1} \gamma_{2}}}{\left(\gamma_{1}+\gamma_{2}-2 i \omega\right)^{2}}
\end{array}
$$


In the following, we will study the effect of passive and active OPO filtering cavities on SPDC modes.

\section{Effect of passive filtering cavity on SPDC modes}

We first examine the effect of passive filtering cavity on the SPDC modes, so $\epsilon \rightarrow 0$, we also consider a symmetric cavity with equal loss rate at each mirror, $\gamma_{1}=\gamma_{2}=\gamma$, thus equations of motion simplify to:

$$
\begin{aligned}
a_{\text {out }}\left(\omega+\omega_{0}\right) & =\frac{i \omega a_{\text {in }}\left(\omega+\omega_{0}\right)+\gamma b_{\text {in }}\left(\omega+\omega_{0}\right)}{\gamma-i \omega} \\
b_{\text {out }}\left(\omega+\omega_{0}\right) & =\frac{i \omega b_{\text {in }}\left(\omega+\omega_{0}\right)+\gamma a_{\text {in }}\left(\omega+\omega_{0}\right)}{\gamma-i \omega}
\end{aligned}
$$

Thus the filtering cavity acts like a frequency dependent two-ported beam splitter. At resonance frequency $\omega=0$

$$
b_{\text {out }}\left(\omega_{0}\right)=a_{\text {in }}\left(\omega_{0}\right)
$$

It is a bandpass filter and passes all the light at the resonance frequency. But at frequencies far from resonance $\omega>>\gamma$ :

$$
\begin{gathered}
a_{\text {out }}\left(\omega_{0}\right)=-a_{\text {in }}\left(\omega_{0}\right) \\
b_{\text {out }}\left(\omega_{0}\right)=-b_{\text {in }}\left(\omega_{0}\right)
\end{gathered}
$$

So all light is reflected with the well known $\pi$ phase shift at these frequencies.

Now, we consider a filtering cavity with an SPDC photon as input to the filter 
from left, whose wave function can be written as:

$$
|\psi\rangle_{i n}=\int d \omega f(\omega) a_{i n}^{\dagger}\left(\omega+\omega_{0}\right)|0\rangle_{a}|0\rangle_{b}
$$

where $f(\omega)$ is the SPDC frequency spectrum, using the inverted form of Eq. (2.24) as:

$$
a_{i n}^{\dagger}\left(\omega+\omega_{0}\right)=\frac{\gamma b_{\text {out }}^{\dagger}\left(\omega+\omega_{0}\right)+i \omega a_{\text {out }}^{\dagger}\left(\omega+\omega_{0}\right)}{\gamma+i \omega}
$$

We can evolve the state through the cavity [45] giving:

$$
|\psi\rangle_{\text {out }}=U|\psi\rangle_{\text {in }}=\int d \omega f(\omega) \frac{\gamma b_{\text {out }}^{\dagger}\left(\omega+\omega_{0}\right)+i \omega a_{\text {out }}^{\dagger}\left(\omega+\omega_{0}\right)}{\gamma+i \omega}|0\rangle_{a}|0\rangle_{b}
$$

And so,

$$
|\psi\rangle_{\text {out }}=\int f(\omega) d \omega \frac{\gamma}{\gamma+i \omega}|0\rangle_{a}|1\rangle_{b}+\frac{i \omega}{\gamma+i \omega}|1\rangle_{a}|0\rangle_{b}
$$

Therefore, the single photon is in a superposition of being reflected $|1\rangle_{a}|0\rangle_{b}$ or transmitted $|0\rangle_{a}|1\rangle_{b}$ by the cavity, with frequency dependent probabilities. If we consider the reflected field to be lost then the transmitted field is in a mixed state of the vacuum and a single photon state with the Lorentzian frequency distribution:

$$
\frac{\gamma^{2}}{\gamma^{2}+\omega^{2}}
$$

Now, we consider both the signal and idler photons into cavity, each of them can be treated independently with respective frequencies $\omega_{s}=\omega$ and $\omega_{i}=-\omega$, so:

$$
|\psi\rangle_{i n}=\int f(\omega) d \omega a_{i n_{s}}^{\dagger}\left(\omega_{0}+\omega\right) a_{i n_{i}}^{\dagger}\left(\omega_{0}-\omega\right)|0\rangle_{a_{s}}|0\rangle_{b_{s}}|0\rangle_{a_{i}}|0\rangle_{b_{i}}
$$


and

$$
\begin{gathered}
|\psi\rangle_{\text {out }}=\int f(\omega) d \omega a_{\text {out }_{s}}^{\dagger}\left(\omega_{0}+\omega\right) a_{\text {out }_{i}}^{\dagger}\left(\omega_{0}-\omega\right)|0\rangle_{a_{s}}|0\rangle_{b_{s}}|0\rangle_{a_{i}}|0\rangle_{b_{i}} \\
=\int f(\omega) d \omega\left(\frac{\gamma b_{\text {out }}\left(\omega_{0}+\omega\right)+i \omega a_{\text {outs }}^{\dagger}\left(\omega_{0}+\omega\right)}{\gamma+i \omega}\right)\left(\frac{\gamma b_{\text {out } i}^{\dagger}\left(\omega_{0}-\omega\right)-i \omega a_{\text {out } i}^{\dagger}\left(\omega_{0}-\omega\right)}{\gamma-i \omega}\right)|0\rangle_{a_{s}}|0\rangle_{b_{s}}|0\rangle_{a_{i}}|0\rangle_{b_{i}}
\end{gathered}
$$

Thus,

$$
\begin{aligned}
|\psi\rangle_{\text {out }}=\int f(\omega) d \omega & \left(\frac{\gamma^{2}}{\gamma^{2}+\omega^{2}}|0\rangle_{a_{s}}|1\rangle_{b_{s}}|0\rangle_{a_{i}}|1\rangle_{b_{i}}\right. \\
& -\frac{i \omega \gamma}{\gamma^{2}+\omega^{2}}|0\rangle_{a_{s}}|1\rangle_{b_{s}}|1\rangle_{a_{i}}|0\rangle_{b_{i}} \\
& +\frac{i \omega \gamma}{\gamma^{2}+\omega^{2}}|1\rangle_{a_{s}}|0\rangle_{b_{s}}|0\rangle_{a_{i}}|1\rangle_{b_{i}} \\
& \left.+\frac{\omega^{2}}{\gamma^{2}+\omega^{2}}|1\rangle_{a_{s}}|0\rangle_{b_{s}}|1\rangle_{a_{i}}|0\rangle_{b_{i}}\right)
\end{aligned}
$$

Therefore, signal and idlers are in a superposition of four output states upon hitting the cavity. They both pass through the cavity with a probability of $\left(\frac{\gamma^{2}}{\gamma^{2}+\omega^{2}}\right)^{2}$, this probability is higher for photons with near degenerate frequencies, there is also a probability of $\left(\frac{\omega^{2}}{\gamma^{2}+\omega^{2}}\right)^{2}$ where both the signal and idler reflect from the cavity, which is high for photons far from the resonance frequency. These two cases are of interest for us as the reason for implementing the cavity is to extract photons with near resonance frequencies and block off resonant ones. As clear from Eq. 2.34, there is also a possibility of $2\left(\frac{\gamma \omega}{\gamma^{2}+\omega^{2}}\right)^{2}$ where one of the signal or idler passes while the other one rejects by the cavity, this case is not desirable since it cause a loss in the photon number correlation. This loss is a Lorentzian loss and is due to the Lorentzian 
decay linewidth of the cavity. The maximum photon number correlation efficiency is therefore bounded by the Lorentzian loss.

We look at the spectrum of the beam on the signal or idler detector placed after filtering cavity on the $b_{\text {out } s, i}$ mode:

$$
S(\omega)=\left\langle 1,\left.0\right|_{a, b} b_{\text {out }}^{\dagger} b_{\text {out }} \mid 1,0\right\rangle_{a, b}=\frac{\gamma^{2}}{\gamma^{2}+4 \omega^{2}}\left\langle 1,\left.0\right|_{a, b} a_{\text {in }}^{\dagger} a_{\text {in }} \mid 1,0\right\rangle_{a, b}=\frac{\gamma^{2}}{\gamma^{2}+4 \omega^{2}}
$$

Therefore the full width at half maximum (FWHM) of the spectrum, $\Delta \omega$ for each of the signal or idler fields is simply $\Delta \omega=0.64 \gamma$. And the total photon number on each detector is proportional to:

$$
\int_{-\infty}^{\infty} f(\omega) d \omega S(\omega)
$$

The spectrum of the coincident detection $S(\omega)_{C}$ is:

$$
S(\omega)_{\text {coincidence }}=\left\langle b_{\text {outs }}^{\dagger} b_{\text {outs }} b_{\text {out } i}^{\dagger} b_{\text {out } i}\right\rangle=\frac{\gamma^{4}}{\left(\gamma^{2}+4 \omega^{2}\right)^{2}}
$$

vs the spectrum of singlet measurements on one of the detectors, for example click on the signal when idler photon is reflected:

$$
S(\omega)_{\text {singlet }}=\left\langle b_{\text {outs }}^{\dagger} b_{\text {out } s} a_{\text {out } i}^{\dagger} a_{\text {out } i}\right\rangle=\left(\frac{\gamma \omega}{\gamma^{2}+4 \omega^{2}}\right)^{2}
$$

Therefore, correlation efficiency $e$, the number of coincidences on the signal and idler 
detectors divided by the average number of photons on each detector, can be calculated as follow:

$$
e=\frac{\int_{-\infty}^{\infty} d \omega f(\omega) S_{\text {coincidence }}}{\int_{-\infty}^{\infty} d \omega f(\omega) S_{\text {singles }}+\int_{-\infty}^{\infty} d \omega f(\omega) S_{\text {coincidence }}}
$$

For the SPDC photon with a frequency spectrum of $f(\omega)=1$, for $-\frac{B}{2}<\omega<\frac{B}{2}$ and $f(\omega)=0$ for $|\omega|>\frac{B}{2}$, then:

$$
e=\frac{\int_{-\frac{B}{2}}^{\frac{B}{2}} d \omega \frac{\gamma^{4}}{\left(\gamma^{2}+4 \omega^{2}\right)^{2}}}{\int_{-\frac{B}{2}}^{\frac{B}{2}} d \omega\left(\frac{\gamma \omega}{\gamma^{2}+4 \omega^{2}}\right)^{2}+\int_{-\frac{B}{2}}^{\frac{B}{2}} d \omega \frac{\gamma^{4}}{\left(\gamma^{2}+4 \omega^{2}\right)^{2}}}
$$

For $B>>\gamma$,

$$
e \longrightarrow \frac{1}{2}
$$

Therefore due to the Lorentzian decay on the filtering cavity, the photon number correlation efficiency cannot get beyond 50\%.

Therefore, we have shown that the filtering cavity can perfectly reduce the SPDC bandwidth to the cavity BW, $\Delta \omega=\gamma$, but note that Fabry-Perot spectrum repeats itself every FSR, so one needs to carefully design the filtering cavity in a way that its FSR be greater than the SPDC generation bandwidth to make sure only one resonance frequency exists in the SPDC spectrum. 


\section{Time domain analysis of filtering cavity}

Equivalently in time domain, SPDC photons are short pulses with a coherence time

of $\delta t=\frac{1}{\triangle \omega_{S P D C}}=\frac{1}{B}$. Photons enter the cavity, and leave after several roundtrips, some of them will pass right away but others will stay longer and then leave after few roundtrips, the decay rate is exponential with the time constant proportional to the cavity storage time $\Delta t=\frac{1}{\Delta \omega_{\text {cavity }}}$.

So the output of the cavity will be a train of $\delta t$ pulses in a $\Delta t$ envelope, when each of $\delta t$ pulses are separated by the cavity round trip time $t_{r}=\frac{1}{F S R}$, Fig. 2.20 .

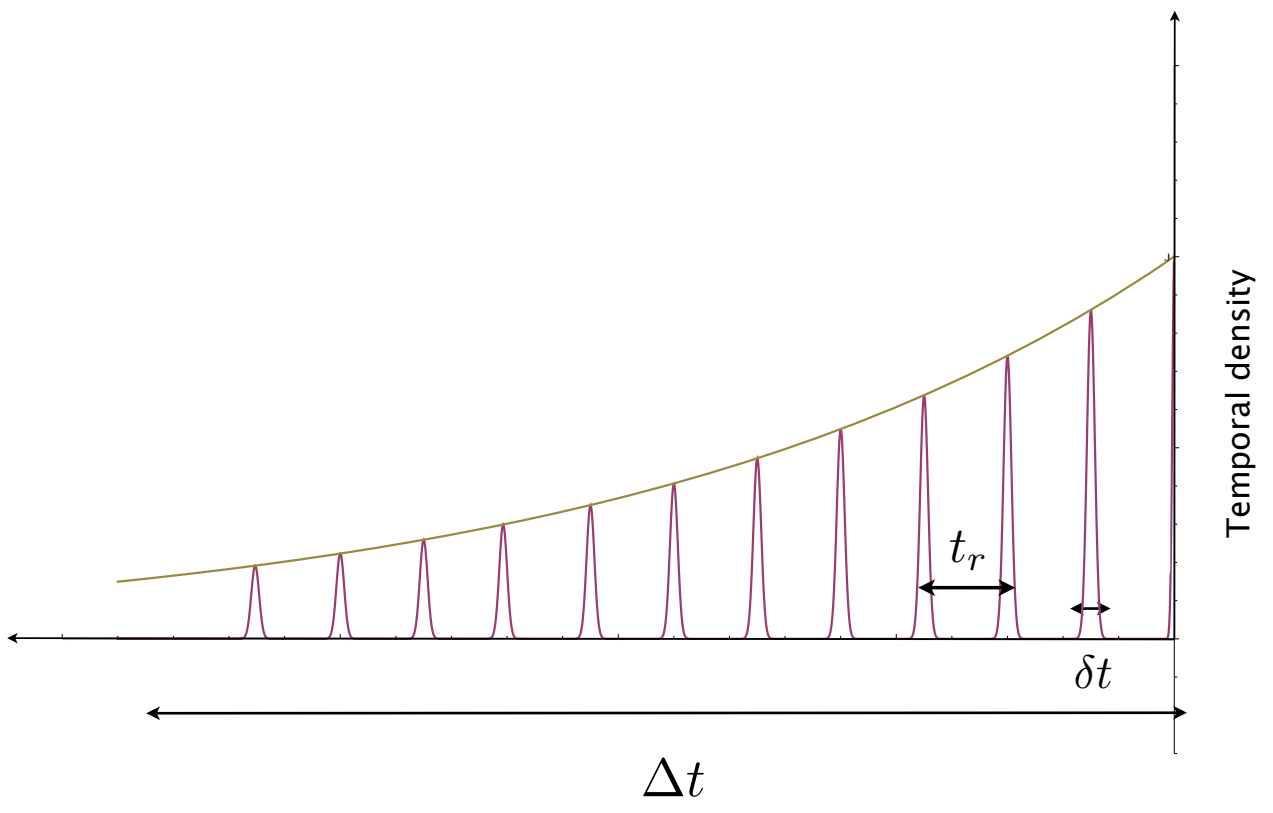

Figure 2.20: Intensity of the resonant SPDC field transmitted by the cavity, versus time

However, if we force the filtering cavity FSR to satisfy $F S R>B / 2$ then it means $\delta t>\frac{t_{r}}{2}$ and so the train of discerete pulses will merge to the continuous long pulse 
of $\Delta t$. So one can increase the temporal bandwidth or equivalently decrease the frequency BW of the SPDC modes by placing a passive filtering cavity with large FSR. Increasing the temporal mode of the SPDC source is of great interest for us, since TES detectors are slow with long temporal mode, so one need to adjust the temporal mode of the source to match it with detectors temporal mode.

\section{Effect of active OPO filter on SPDC modes}

Now, we consider the effect of active filtering cavity on SPDC modes, this time the down converting crystal is placed inside an one-sided cavity with loss rate $\gamma_{1}=\gamma$ and $\gamma_{2}=0$ and $\epsilon \neq 0$ in Eq. (2.23).

Collet and Gardiner [43] showed that placing a down converting crystal inside a single-ended cavity produces a maximally entangled photon pair and optimizes the photon number correlation. In fact, SPDC photons generated in a two-sided cavity can penetrate either mirror, so photon number correlation degrades when measured at only one output port of the cavity say $b_{\text {out }}$. In contrast, placing a parametric amplifier inside a single-ended cavity forces all photons to appear at the single output of the cavity and produces maximally correlated photon pair.

Therefore, Eq. (2.23) simplifies to:

$$
a_{\text {out }}\left(\omega_{0}+\omega\right)=G_{1}^{\prime}(\omega) a_{\text {in }}\left(\omega_{0}+\omega\right)+g_{1}^{\prime}(\omega) a_{\text {in }}^{\dagger}\left(\omega_{0}-\omega\right)
$$


where

$$
G_{1}^{\prime}(\omega)=\frac{\gamma+2 i \omega}{\gamma-2 i \omega}, \quad g_{1}^{\prime}(\omega)=\frac{4 \epsilon \gamma}{(\gamma-2 i \omega)^{2}}
$$

The field spectrum of resonant SPDC modes on the cavity output is:

$$
\left\langle a^{\dagger}\left(\omega_{0}+\omega\right) a\left(\omega_{0}+\omega^{\prime}\right)=S^{\prime}(\omega) \delta\left(\omega-\omega^{\prime}\right)\right.
$$

where:

$$
S^{\prime}(\omega)=\left|g_{1}(\omega)\right|^{2}=\frac{16|\epsilon|^{2} \gamma^{2}}{\left(\gamma^{2}+4 \omega^{2}\right)^{2}}
$$

Therefore, similar to the passive filtering case the full width at half maximum (FWHM) of the spectrum from the down-converted field is simply $\Delta \omega=0.64 \gamma$, cavity reduces the SPDC bandwidth to the BW defined by the cavity. Cavity resonant fields are also amplified by a factor of $\sim \frac{1}{\gamma^{2}}$, which will cause enhancement effect in downconversion due to cavity resonance.

The overall signal level is proportional to:

$$
R_{\text {resonance }} \sim \int_{-\infty}^{\infty} d \omega S^{\prime}(\omega)=\int_{-\infty}^{\infty} d \omega \frac{16|\epsilon|^{2} \gamma^{2}}{\left(\gamma^{2}+4 \omega^{2}\right)^{2}}=\frac{4\left|\epsilon^{2}\right| \pi}{\gamma}=8|r|^{2} F^{2} \Delta \omega
$$

Where $r=\epsilon t_{r}$ is a single pass gain parameter, with $t_{r}=\frac{1}{F S R}$ as cavity round trip time, and $F$ the cavity Finesse $F=\frac{F S R}{\Delta \omega}=\frac{1}{\Delta \omega t_{r}}$.

To calculate the cavity enhancement factor, as was first calculated by Ou's group [42], we need to compare the signal level for the cavity enhanced doubly resonant 
modes where both of the signal and idlers are resonating in the cavity, with the signal level for non resonant SPDC modes. In fact not all the signal and idler modes of the multimode SPDC are resonant to the cavity. Many of them are not mode matched to the cavity or have frequencies far from resonance and will leak out of the cavity after few bounces and without any enhancement.

The signal rate for free space SPDC generation:

$$
S^{\prime \prime}(\omega)=g^{\prime}(\omega)=r f(\omega)
$$

where $f(\omega)$ is a gain spectrum of single-pass spontaneous down conversion determined by phase matching condition, so the overall single pass generation is:

$$
R_{\text {single-pass }} \sim \int_{-\infty}^{\infty} d \omega S^{\prime \prime}(\omega)=\int_{-\infty}^{\infty} d \omega|r|^{2} f(\omega)^{2} \Delta \omega \sim \Delta \omega_{S P D C}|r|^{2}
$$

and so the average enhancement factor $E F$ per mode is:

$$
E F=\frac{R_{\text {resonance }} / \Delta \omega_{\text {cavity }}}{R_{\text {Single-pass }} / \Delta \omega_{S P D C}} \sim F^{2}
$$

Therefore the average enhancement effect per mode is proportional to $F^{2}$, or roughly is equivalent to the square of the number of bounces of light before it leaves the cavity. Note that the $E F$ is the cavity enhancement factor per mode and indicates the ratio of SPDC photons emitted in the cavity mode compare to photons emitted in random non resonant field modes in a frequency interval near cavity resonance frequency. This cavity enhancement is of great interest because it forces most of the 
SPDC photons to be emitted to the spatially and spectrally well-defined cavity mode.

However, observing this enhancement effect is not trivial in practice, as we do not have ideal single frequency photo-detectors to only select SPDC modes with resonant frequency. In fact, all practical photo-detectors have relatively large detection BW, so they will detect all modes generated in a whole SPDC spectrum, so the measured

$\mathrm{EF}$ is degraded by $\frac{\Delta \omega_{\text {cavity }}}{\Delta \omega_{S P D C}}$. To increase the observed EF and in order to clearly see the enhancement effect we need to place another filtering cavity after active cavity to decrease the $\Delta \omega_{S P D C}$. In other words enhanced doubly resonant modes of the cavity are buried in the ocean of non resonant modes, so one need to reject many of the non resonant modes to increase the ratio of resonant to non resonant modes and improve the enhancement effect.

\subsubsection{Experimental observation of the cavity enhancement and the onset of stimulated emission in an OPO oper- ating well below the oscillation threshold}

In this section, I focus on our efforts towards generating cavity-enhanced spontaneous parametric down conversion. We built an intrinsically stable optical parametric oscillator (OPO) and showed that well-defined cavity modes enable us to herald single photons in narrowband and single-mode quantum states, with up to $80 \%$ efficiency in preliminary results, which is the highest heralding ratio we have achieved so far after trying many different approaches.

The OPO is a Fabry-Perot cavity consisting of two opposing spherical mirrors, Fig. 2.21. In our experiment we designed an $\mathrm{OPO}$ with $5 \mathrm{~cm}$ focal length mirrors and 


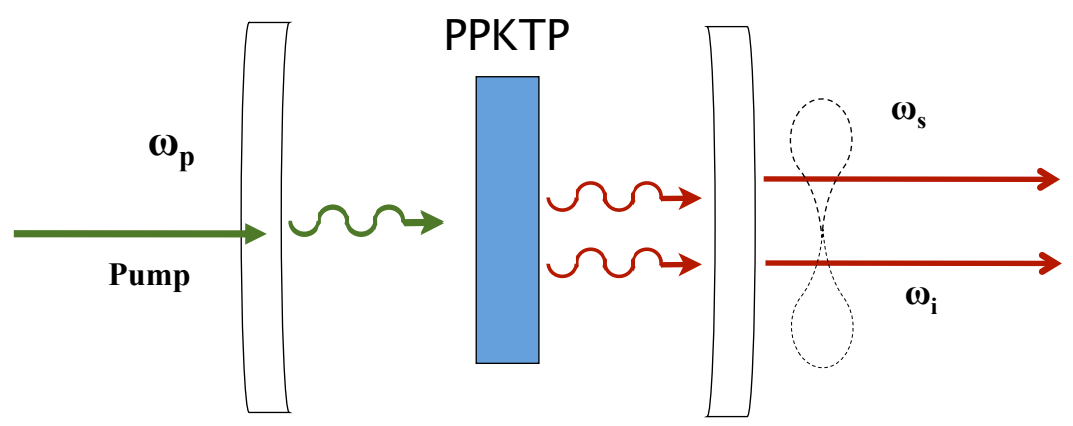

Figure 2.21: Schematic of the Optical Parametric Oscillator (OPO), pumped with pump photons with $\omega_{p}$ and generated signal and idler photons with $\omega_{s}$ and $\omega_{i}$

$\mathrm{L}=10 \mathrm{~cm}$ mirror separation distance. A PPKTP crystal was placed inside the cavity and was pumped with $532 \mathrm{~nm}$ laser, with pump photons down converting into photonnumber-correlated signal and idler modes in the infrared (IR) wavelength range ( 1064 $\mathrm{nm})$. The OPO is a single-ended cavity, in which one of the cavity mirrors is highly reflective but the other one has $2 \%$ transmissivity for IR. The input cavity mirror is highly transmissive at $532 \mathrm{~nm}$, and the output cavity mirror is almost totally reflective at $532 \mathrm{~nm}$ so pump mode does not resonate inside the cavity and leaves the cavity after a double pass. The cavity has a finesse of $F=314$, with a Free Spectral Range (FSR) of $1.5 \mathrm{GHz}$ and a bandwidth close to $10 \mathrm{MHz}$.

Optical parametric oscillators are well known sources of nonclassical light and have been already used to generate single-mode [46 and two-mode squeezed states 47]. It is very well studied that resonating both the signal and idler fields simultaneously in a doubly resonant OPO operating near and below the oscillation threshold, produces a significant enhancement in the nonlinear interaction, as the cavity also confines signals 
and idlers to well defined spatial modes and dramatically narrows their bandwidth [48], [49], [50].

When the OPO is operating near the oscillation threshold, the cavity enhancement is very high and the ratio of doubly resonant photons, where both the signal and idler modes are simultaneously resonant in the cavity, to non resonant ones, where non of them are resonant, is huge and so one can completely ignore down conversion into non resonant modes. In this regime enhancement is mostly due to the process of the stimulated parametric down conversion.

There exist three different regimes of operation for an OPO, above the oscillation threshold, where OPO "lases" in bright and well-defined signal and idler modes, below the oscillation threshold where stimulated emission process is still dominant. And well below the oscillation threshold in which stimulated emission is negligible and all photons are generated through the SPDC process.

The generation rate of the doubly resonant stimulated emission is proportional to $G^{2}$, where $G$ is the pump power level normalized to the pump threshold power [50], [51]. When the OPO is operating above the oscillation threshold, $G>1$, the signal and idler generation rate is higher than the cavity loss rate, therefore OPO lases and generates bright signal and idler modes. But when OPO operates below the oscillation threshold $G<1$, stimulated emission process still dominates the spontaneous process, but not cavity losses, the OPO does not lase and does not produce a bright output. The stimulated emission rate decreases with the square of the pump power, so in 
OPOs operating well below the oscillation threshold, $G \simeq 1 \%$ the stimulated emission rate is remarkably degraded and most photons are generated through the process of spontaneous down conversion.

\section{OPO operating above oscillation threshold}

It is well known that, in a doubly resonant type-II OPO, the double-resonance condition for the signal and idler inside a common cavity containing a birefringent nonlinear crystal leads to a densely clustered mode structure [48, 49]

The cluster mode structure of our doubly resonant OPO, operating above the oscillation threshold is shown in Fig. 2.22. In this oscilloscope trace, we measured the pump beam reflected from cavity and the cluster structure is on the pump depletion, which is due to conversion of the pump power into signal and idler modes. The trace shows the rejected pump from OPO vs the cavity length detuning generated by a piezoelectric transducer (PZT) mounted on one of the mirrors.

At double resonance both the signal and idler modes should satisfy:

$$
L+n_{s, i} l=p_{s, i} \frac{\lambda_{s, i}}{2}=p_{s, i} \frac{c}{2 \nu_{s, i}}
$$

where $n_{s, i}$ is the refraction indices of the signal and idler modes, $\mathrm{L}$ is the cavity length outside the crystal, $l$ the crystal length, $p_{s, i}$ their respective mode numbers (positive integers) and $\nu_{s, i}$ is the signal and idler frequencies, which should also satisfy the conservation of energy $\nu_{s}+\nu_{i}=\nu_{p}$. 


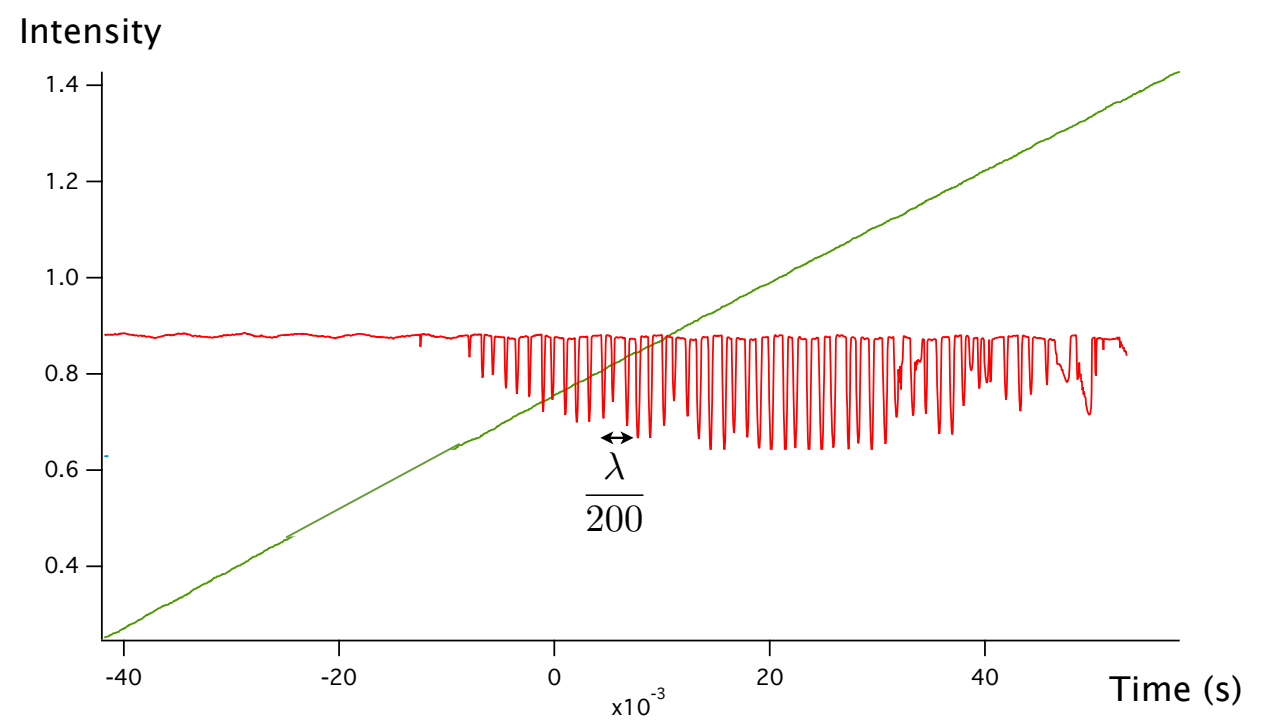

Figure 2.22: Red Pump depletion peaks for doubly resonant modes well above the OPO threshold. Green, ramp signal applied to a piezzo mirror of the OPO.

The minimum cavity length displacements corresponding to mode hops are calculated by Sheng Feng et al in [48] as:

$$
\delta L_{\text {min }} \simeq\left[\left(\delta p_{s}+\delta p_{i}\right)+\frac{\delta n}{2(\bar{n}+L / l)}\left(\delta p_{s}-\delta p_{i}\right)\right] \frac{\lambda_{p}}{2}
$$

where $\lambda$ is the pump wavelength and $\bar{n}=\frac{\left(n_{s}+n_{i}\right)}{2}$. Mode clusters are labelled by $\left(\delta p_{s}+\delta p_{i}\right)$ and are separated by half a pump wavelength. Inside a given cluster, the modes are labelled by $\left(\delta p_{s}+\delta p_{i}\right)$ and separated by $\left(\delta p_{s}=-\delta p_{i}= \pm 1\right)$, then:

$$
\delta L_{\text {min }} \simeq \frac{\delta n \lambda_{p}}{2(\bar{n}+L / l)}<<\lambda_{p}
$$

In our experiment, $\delta L_{\min }$ is in the order of $\lambda / 200$ so mode hops happen at every $\simeq 2.2 \mathrm{~nm}$. This should be compared to the usual $\lambda / 2$ mode hop of a singly resonant OPO, e.g, laser cavity. Doubly resonant OPOs are extremely delicate to stabilize. 
We then locked the cavity on double resonance using Pound-Drever-Hall locking technique using the depleted pump signal as the cavity-length error signal, few percent pump depletion is enough for locking. Bright signal and idler lasing modes are generated in a well-defined cavity modes and are used for alignment and mode matching of cavity modes into fibers and other optical elements.

\section{OPO below the oscillation threshold and observation of onset of stimulated emission}

Now, we briefly study the behavior of OPO operating below the oscillation threshold. We first brought the OPO above threshold and then dropped the pump power to the level of $P_{\text {pump }}=0.1 P_{t h}$, where $P_{t h}=250 \mathrm{~mW}$. We observed the cluster structure on the TES raw data, as were slowly tuning the cavity length, Fig. 2.23 .
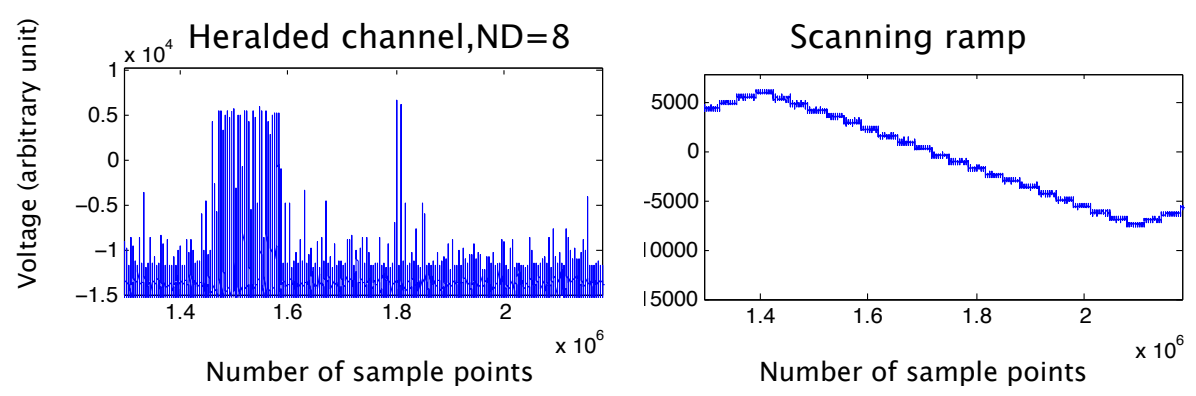

Figure 2.23: ]

Doubly resonant peaks on the OPO operating below the oscillation threshold, $P_{\text {pump }}=0.1 P_{t h}$ on left, and piezo ramp on the right.

In order to confirm what we observed below threshold was indeed the OPO cluster spectrum, we compared cluster mode structures below and above the oscillation threshold: we tuned the cavity with the same ramp in both cases and then recorded 
each clusters separately, Fig. 2.24. For the above threshold case, we measured doubly resonant modes as pump power depletion on a regular power meter and displayed the signal on the oscilloscope, but when OPO is operating below the oscillation threshold, the emission rate of doubly resonant modes are low, on the order of a nw, so they are not easily detectable by regular power meters. Thus we decided to measure them on TES with a $\mathrm{ND}=8$ optical density filter on the heralded channel to decrease the signal level by a factor of $10^{-8}$ so as to be appropriate for detection on TES. For a reason which will be explained later in this section, we placed a filtering cavity on the heralding channel and looked for doubly resonant structure on the TES detector. Figure 2.24 shows the cluster structure on the heralded and heralding channels along with the piezo scanning ramp.
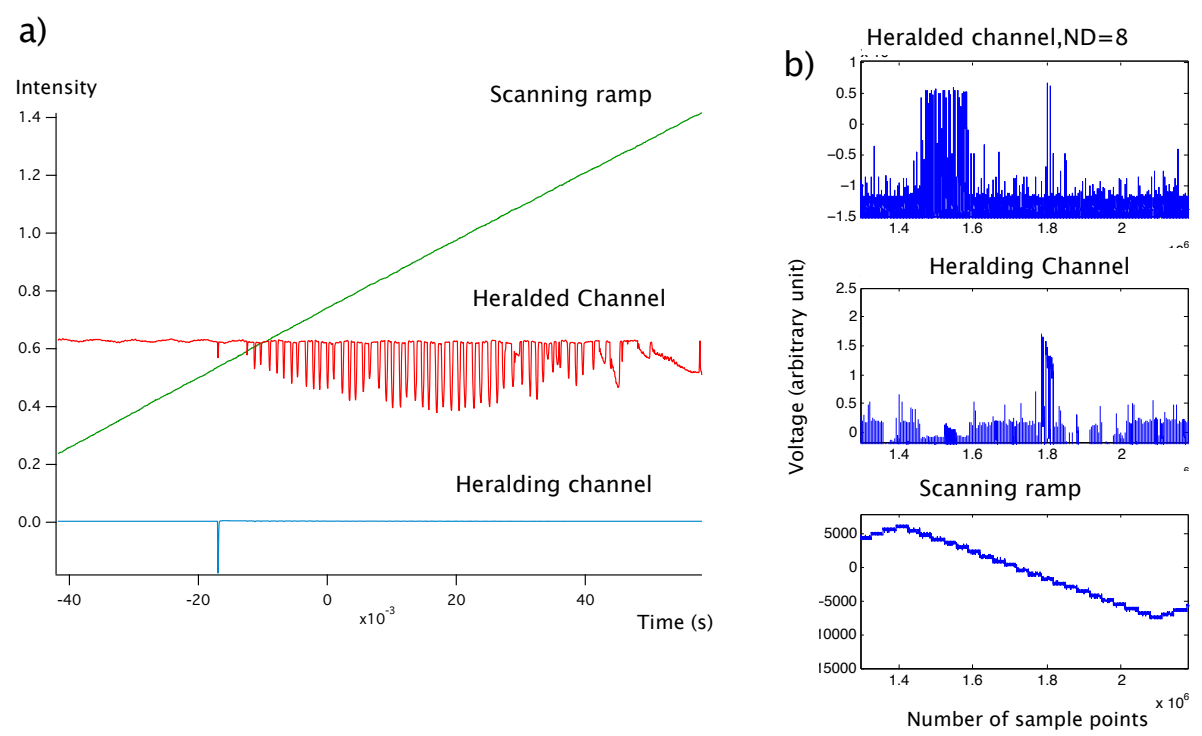

Figure 2.24: Doubly resonant modes a) well above the OPO oscillation threshold, on the oscilloscope, b) below the OPO oscillation threshold, raw TES data 
We then zoomed into the TES raw data and clearly observed the cluster mode structure on the OPO below threshold Fig. 2.25, these clusters are similar to doubly resonant cluster mode structure of OPO operating above threshold.
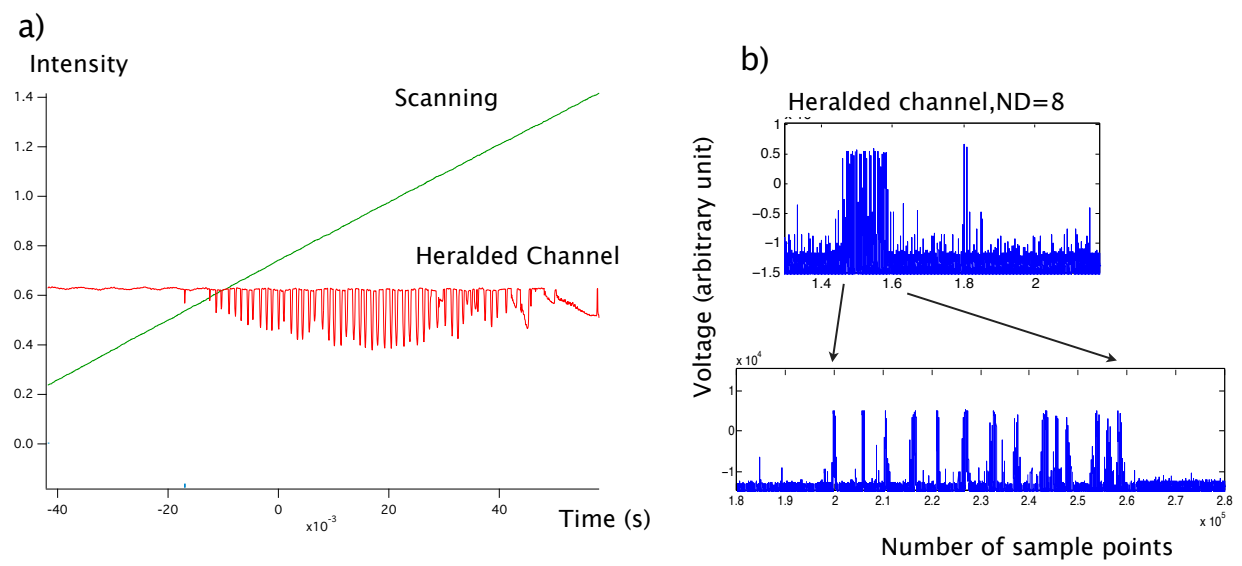

Figure 2.25: Doubly resonant cluster structure on the heralding channel, zoomed in

The piezo ramp speed is the same in both cases, so we can compare two cluster structures by evaluating and comparing their characteristic times.

We define the whole piezo scanning ramp time as $T$, cluster length $T_{C}$, distance between doubly resonant peaks $\Delta T_{c}$ and width of each doubly resonant peak as $\delta t_{c}$ Fig. 2.26.

The ramp time $T$ is equal in both cases, which means the total number of sample points recorded on TES in one full piezo ramp corresponds to the total ramp time $T$, so if we assume 712210 data samples are recorded in $25 \mathrm{~s}$, then relative time between each sample is $35 \mu \mathrm{s}$. Other characteristic times $T_{c}, \Delta T_{c}, \delta T_{c}$ can equivalently be calculated assuming each sample point takes $35 \mu$ s in average, we calculated time 

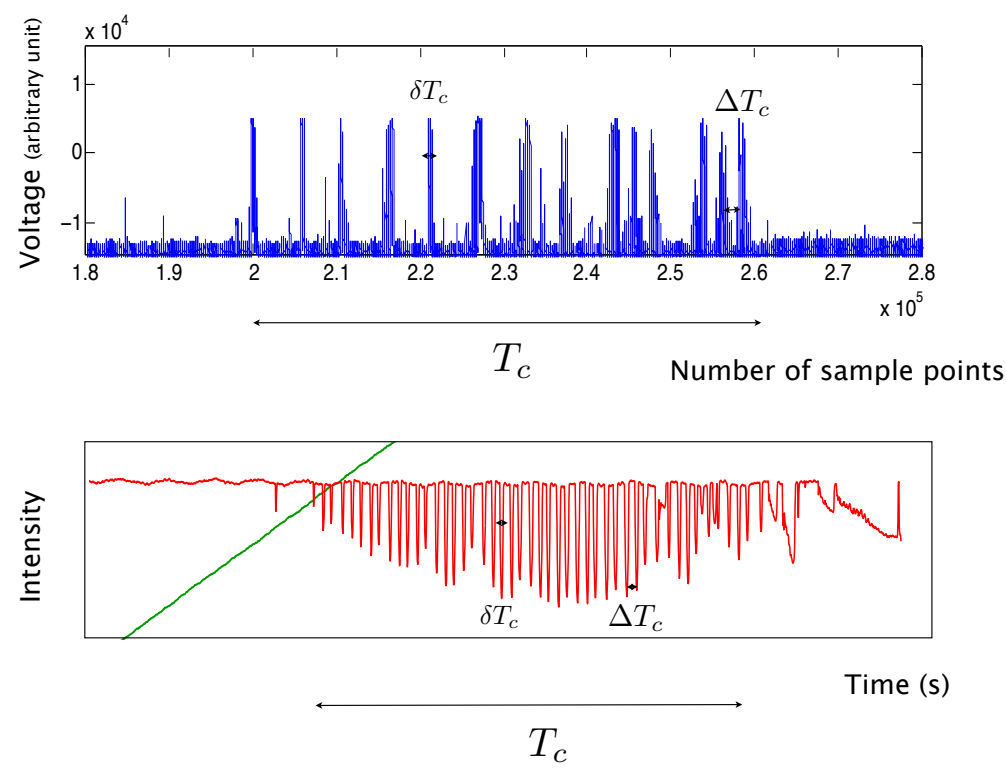

Figure 2.26: Cluster mode structure and its time constants

constants for the above and below threshold and listed them in the following chart, Fig. 2.27. As is clear from the chart, the respective time constants in each case are very close to each other which indicates, as expected, that the doubly resonant mode cluster structure is identical for the OPO below and above the oscillation threshold.

Note that we calculated the average time for each sample point as $35 \mu s$, however as was discussed earlier in 2.2, each sample point actually takes only $400 \mathrm{~ns}$, in fact $35 \mu s$ average time includes the big dead time in the data processing as the Python program acquires data in $13 \mathrm{~ms}$ and then process them during the rest of a second.

As mentioned earlier the stimulated emission rate is proportional to $G^{2}$, the square of the pump power normalized to the pump threshold power, thus decreasing the pump power decreases the stimulated emission rate. Stimulated emission process will 


\begin{tabular}{|c|c|c|c|}
\hline $\begin{array}{c}\text { Time } \\
\text { Distance }\end{array}$ & $\begin{array}{c}\text { timing on } \\
\text { Oscilloscope } \\
(\mathrm{S})\end{array}$ & $\begin{array}{c}\text { Number of } \\
\text { sample points } \\
\text { on TES data }\end{array}$ & $\begin{array}{c}\text { Equivalent } \\
\text { time on TES } \\
(\mathrm{S})\end{array}$ \\
\hline$T$ & 25 & 712210 & 25 \\
\hline$T_{c}$ & 5 & 131620 & 4.62 \\
\hline$\Delta T_{c}$ & 0.1 & 4454 & 0.15 \\
\hline$\delta T_{c}$ & 0.05 & 815 & 0.053 \\
\hline
\end{tabular}

Figure 2.27: Time constants for doubly resonant cluster mode structures below and above the oscillation threshold

eventually disappear and all photons will be emitted through the SPDC process.

Intuitively speaking, for the stimulated emission to happen, one needs enough pump rate to make sure the next pump photon arrives while the signal and idler pair from the previous pump photon is still in the cavity, then the second pump can stimulatedly emit another pair of signal and idler modes in the same spatial mode as the original one, therefore decreasing the pump power reduces this probability. At some point the pump rate will be so low that signal and idler pairs leave the cavity before pump can emit another photon.

We briefly studied the dependence of the stimulated emission rate to the pump power, Fig. 2.28 shows the stimulated emission of doubly resonant peaks for different pump powers ranging from $30 \mathrm{mw}$ down to $8 \mathrm{mw}$.

It is clear from these graphs that as we decrease the pump power the probability of 


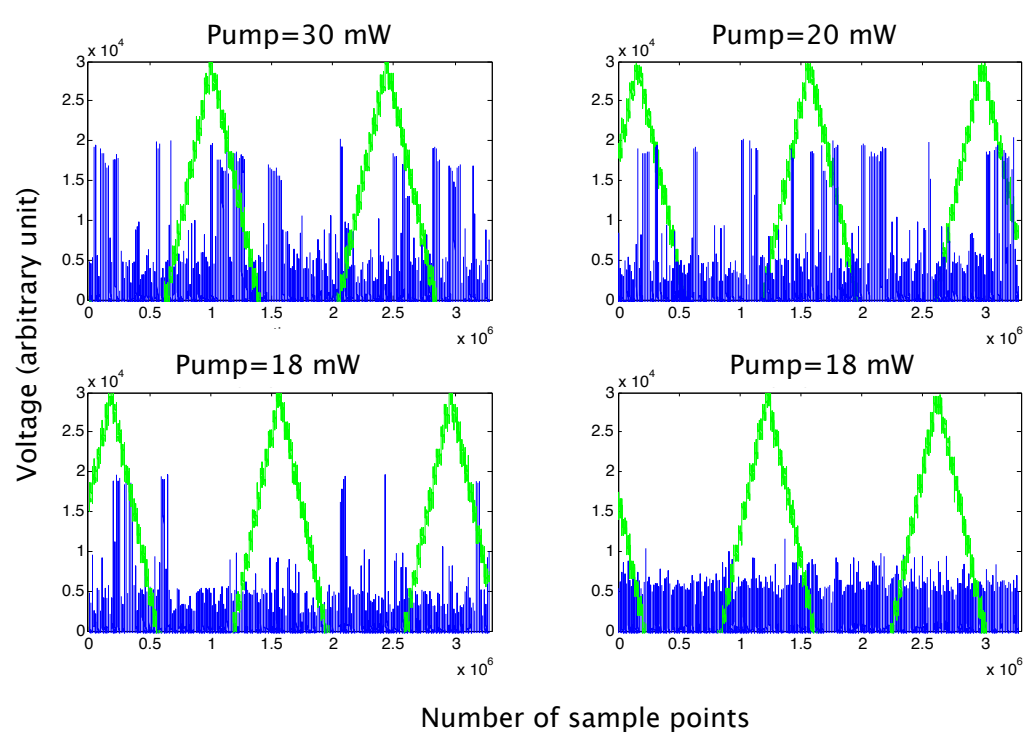

Figure 2.28: Blue, doubly resonant stimulated emission peaks for OPO operating at various pump powers ranging from $30-8 \mathrm{~mW}$. Green, ramp signal applied to a piezzo mirror of the OPO.

stimulated emission decreases, and at $\mathrm{P}=8 \mathrm{~mW}$ stimulated emission peaks completely disappear. We also observed the onset of stimulated emission happening at $\mathrm{P}=18$ $\mathrm{mW}$.

Therefore, we studied OPO operating below the oscillation threshold and observed stimulated doubly resonant cluster structure even at pump powers $P<0.1 P_{t h}$. Emitted signal and idler modes are of great interest as these modes are generated in well-defined cavity modes. However, for experiment in single or multi-photon regimes using TES detectors, one needs to drop the pump power to levels even lower than $0.1 P_{t h}$, for the signal and idler emission rate to be appropriate for measurement on low saturation threshold TES detectors. TES detectors are very slow and their rising 
peak plus cooling time takes $3-4 \mu s$, so one needs to design the photon emission rate to be in the order of few $\mathrm{fW}$, which is equivalent to one photon per $3 \mu \mathrm{s}$. In this regime the OPO is operating well below the oscillation threshold $P<0.01 P_{t h}$, thus we cannot count on cavity enhancement due to stimulated emission and need to find other types of cavity enhancement effects. In the following we studies the cavity enhancement effect due to confining the vacuum modes on OPOs operating well below the oscillation threshold.

\section{OPO operating well-below the oscillation threshold}

As was studied earlier in section 2.4.1 for OPOs operating well below the oscillation threshold some cavity enhancement effect due to the confinement of the vacuum modes exists and results in the enhanced emission of doubly resonant signal and idler modes.

We tried to observe this cavity enhancement effect on TES detectors. We initially brought the OPO above threshold and then dropped the pump power to levels well below the oscillation threshold, then carefully tuned the cavity length to fulfill the doubly resonant condition.But we could not see any enhancement on the signal level as cavity tunes. Fig. 2.29.

In fact the OPO operating well below the oscillation threshold enhances the emission of narrow band doubly resonant signal and idler modes by a factor of $F^{2}$, but it also enhances the generation of narrowband singly resonant modes, where only the signal or idler modes are resonant to the cavity. OPO also emits broadband non res- 


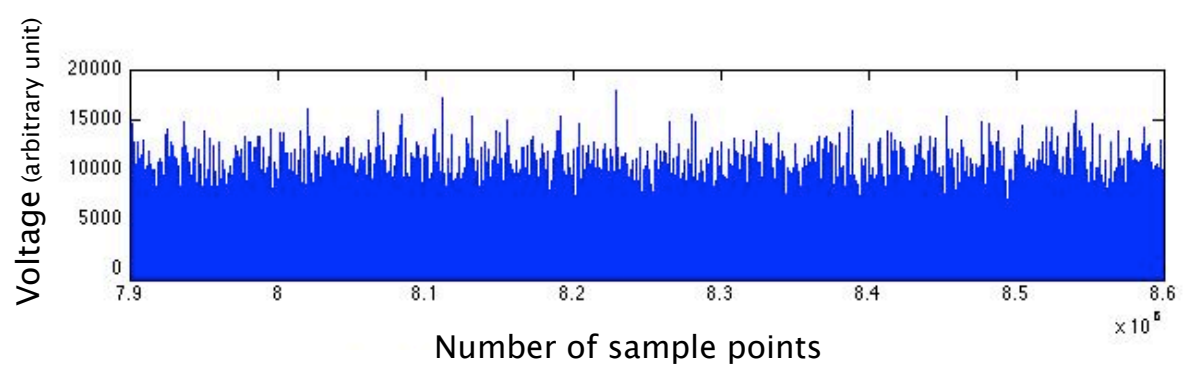

Figure 2.29: Constant signal rate vs cavity length tuning, no sign of doubly resonant effect.

onant modes where none of the signal or idler modes are resonant to the cavity and both will leave the cavity right after generation. This emission mode is, obviously, not enhanced by the cavity.

The condition for the signal or idler modes to resonate in the cavity Eq. (2.52) satisfies for all cavity lengths with corresponding $\nu_{s, i}$ frequencies. These resonant frequencies repeat every free spectral range $\Delta \nu_{F S R}=1.5 \mathrm{GHz}$, so singly resonant signal and idler modes are emitted in frequency combs as shown in Fig. 2.30, Therefore, at any arbitrary length of the cavity, there exist about $10^{3}$ singly resonant modes each with $10 \mathrm{MHz}$ BW. If one divides the whole SPDC frequency spectrum to intervals of $10 \mathrm{MHz}$ BWs and assumes one mode exists in each frequency interval then there exists $10^{3}$ singly resonant modes in a $1 \mathrm{THz}$ SPDC spectrum

Note that combs for orthogonally polarized signal and idler modes do not overlap as each of these modes have different FSR, $n_{s} \neq n_{i}$.

Signal and idler modes are also emitted with non resonant frequencies, these modes don't resonate in the cavity, but are still dominant in the SPDC process as there is 


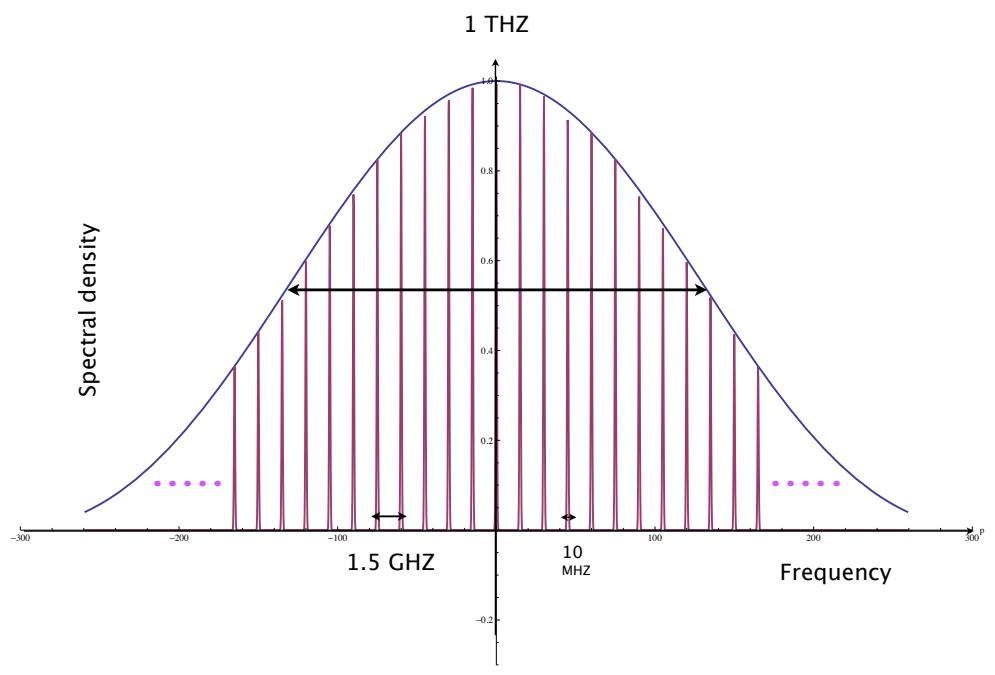

Figure 2.30: Singly resonant frequency comb (pink) for the signal in the SPDC broad frequency spectrum (blue). This graph is not to scale, see text.

overwhelming number of them generated in the huge SPDC bandwidth. For example, assuming one mode in every $10 \mathrm{MHz}$ frequency interval results in the emission of about $10^{5}$ non resonant modes.

For doubly resonant modes, the resonant condition Eq. (2.52) must be satisfied for both the signal and idler modes in a common cavity length $L$, therefore the doubly resonant condition will happen only at special cavity lengths and at each length there only exist one doubly resonant mode Fig. 2.30, note that combs have different FSRs and cannot completely overlap at all frequencies.

Thus even if this one doubly resonant mode enhances by a factor of $F^{2}=10^{5}$, it is still buried in the ocean of singly and non resonant modes and there is no surprise if one cannot directly observe cavity enhancement due to doubly resonant modes by simply tuning the cavity. 


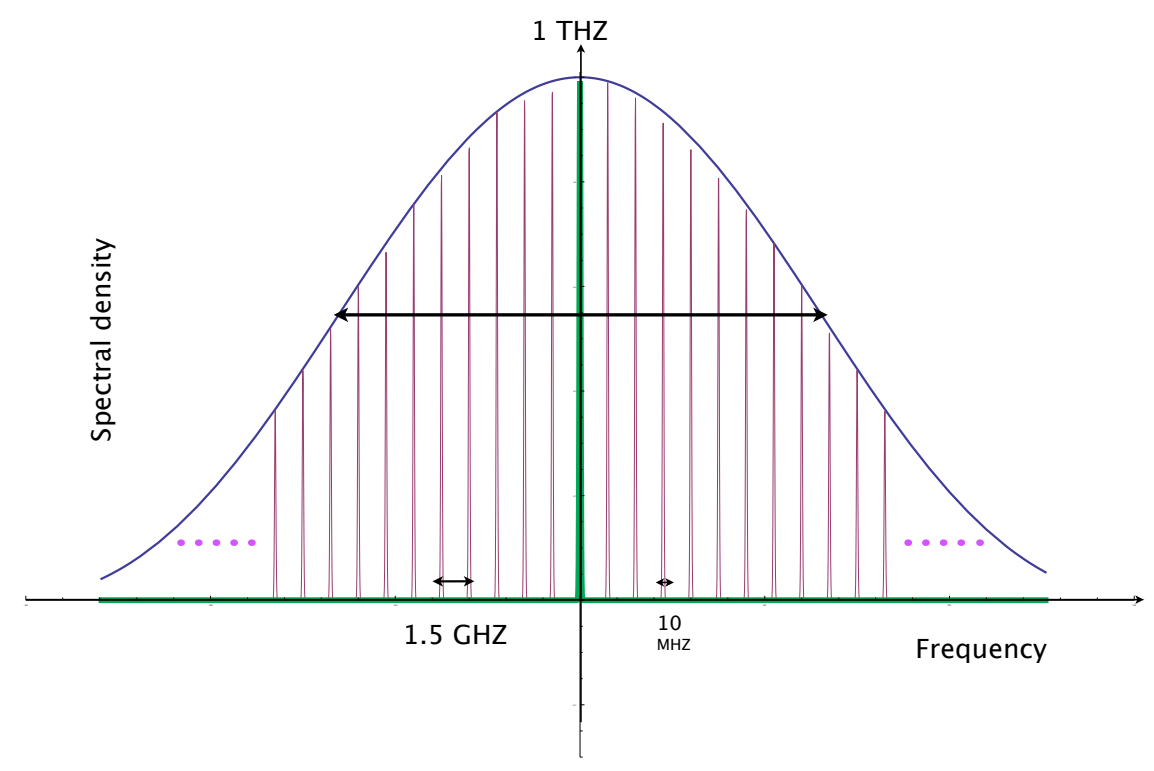

Figure 2.31: ]

Doubly resonant frequency highlighted (green) at the middle of singly resonant frequency comb ( light pink).

\section{Adding a filtering cavity on the heralding channel}

We then decided to place a filtering cavity on the heralding channel to filter out most of the singly and non resonant SPDC modes while passing doubly resonant part of the spectrum. We used the same filtering cavity as was employed earlier for filtering the SPDC modes generated outside an $\mathrm{OPO}$, this cavity has $1 \mathrm{GHz}$ BW which can perfectly transmit doubly resonant modes but filter out modes in the rest of the spectrum Fig. 2.32

This time we placed the filtering cavity only on the heralding channel, Fig. 2.33 . At this point we decided to focus on increasing the heralding ratio and since for the perfect heralding ratio one need to detect a photon on the heralded channel only after 


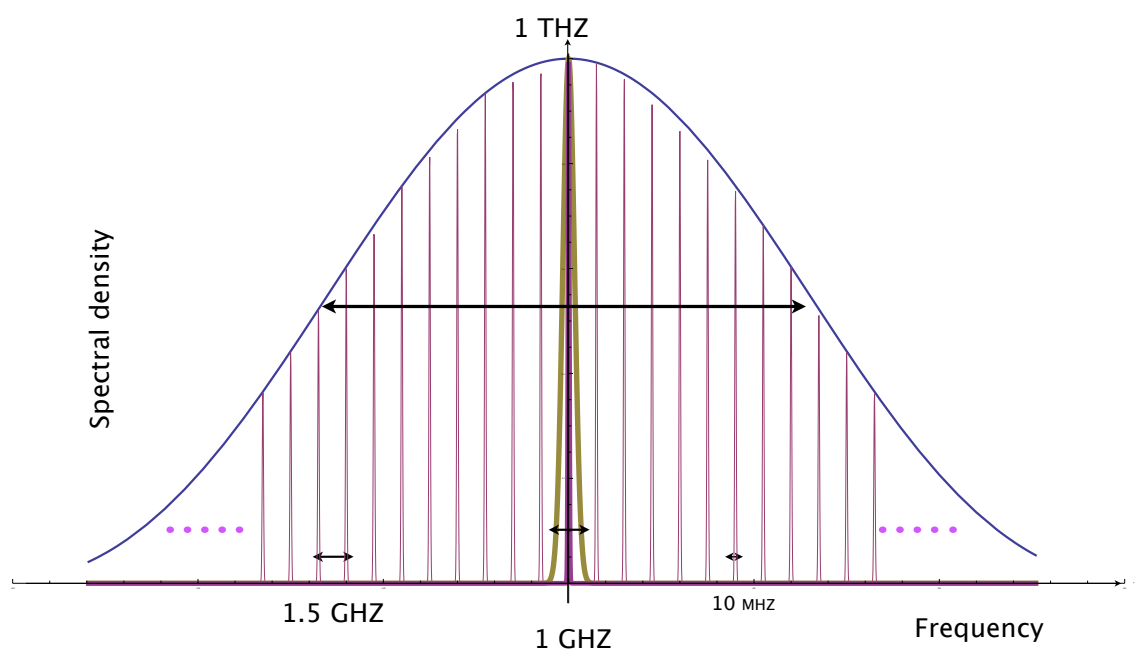

Figure 2.32: ]

Doubly resonant frequency highlighted (dark pink) at the middle of singly resonant frequencies ( light pink), filtering cavity frequency spectrum (yellow) and the SPDC spectrum (blue).

detecting a photon in the heralding detector then the loss on the heralding channel is not important. So we placed the filtering cavity in the heralding channel to avoid the effect of loss due to imperfect mode matching of the filtering cavity.

We then tuned the cavity length slowly by hand while the filtering cavity was locked at the degenerate frequency. Note that the doubly resonant condition does not always satisfied at degenerate frequencies, where $\omega_{s}=\omega_{i}=\frac{\omega_{p}}{2}$.

However, refractive indices $n_{s}$ and $n_{i}$ are temperature dependent and one can tune the crystal temperature to find a specific temperatures where signal and idler modes can be degenerate and both fulfill Eq. (2.52). We likewise tuned the crystal temperature, initially for the OPO above threshold, and noticed that at $T_{\text {crystal }}=$ 


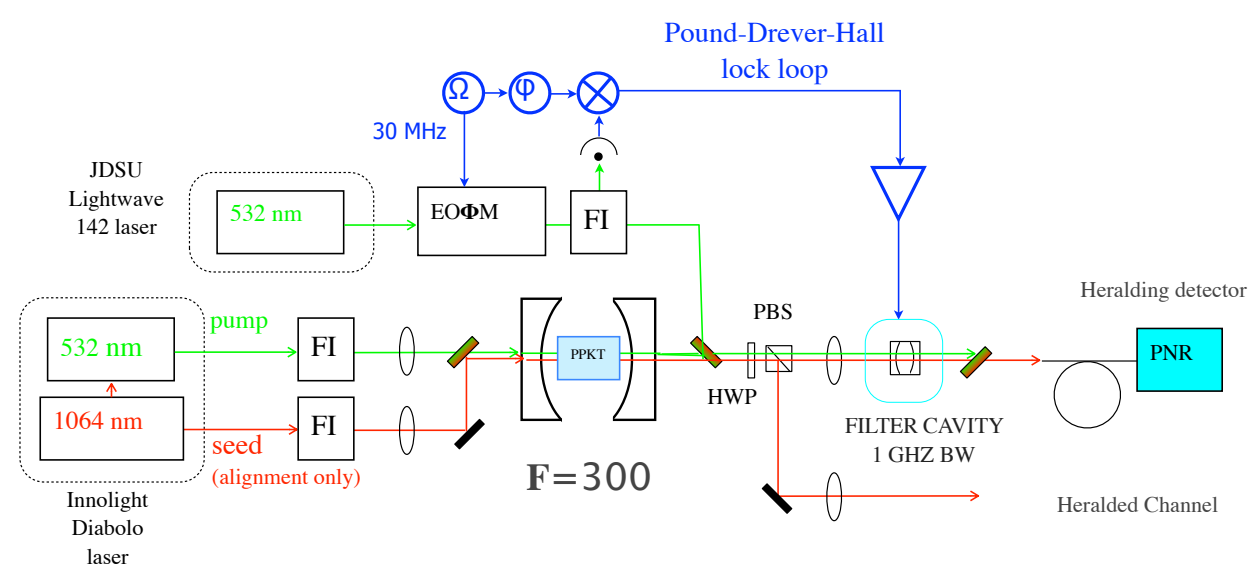

Figure 2.33: Experimental setup, crystal is placed inside a Fabry-Perot cavity and filtering cavity is displaced and is on the heralding channel

$27.29^{\circ}$ one of the doubly resonant modes of the cluster is passing through the cavity.

After finding the right cavity length and the crystal temperatures for degenerate doubly resonant condition based on OPO above threshold, we dropped the power and further fine tuned cavity length and $T$ to compensate for the crystal temperature decrease due to the pump power reduction.

We then observed doubly resonant cavity enhancement effect on the heralding channel Fig. 2.34. Although the heralded channel still has constant output, we also multiplied two channels to have a quick measure of coincidences. The coincidence trace also follow the heralding channel and has increases near doubly resonant frequencies.

So we successfully observed the cavity enhancement effect on doubly resonant modes. As was discussed earlier in this chapter, doubly resonant modes are of great in- 


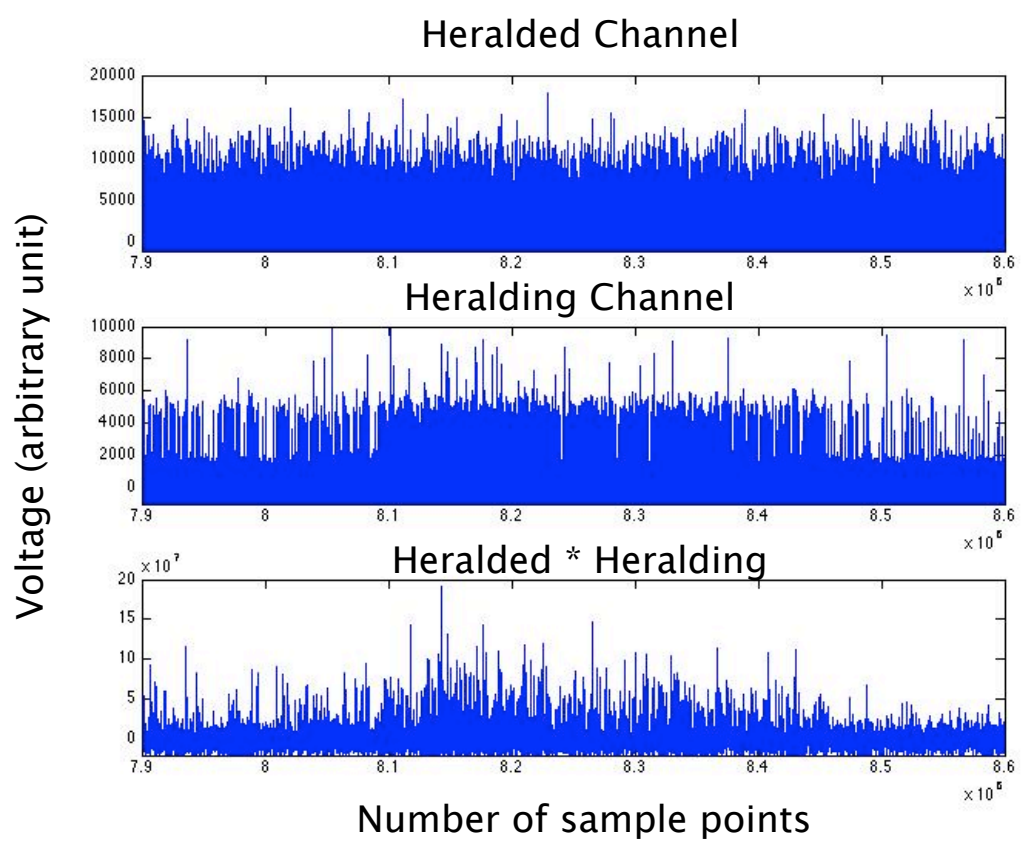

Figure 2.34: TES raw data on the heralding and heralded channels and also their multiplication, cavity enhancement effect is clear.

terest as both the signal and idler modes are simultaneously resonant and enhanced in the cavity.The cavity enhancement effect forces SPDC emission into the well-defined spectral and spatial cavity modes. We expected that concentrating on doubly resonant modes will allow us to reach to higher heralding ratios which is mostly due to efficient collection of well-defined cavity modes into the single mode of the TES fibers.

We then carefully zoomed in the doubly resonant part of the TES raw data and tried to calculate the heralding ratio, Fig. 2.35. In this graph the heralding ratio is as high as $73 \%$, there are 15 photon in the heralding channel and 11 of them coincide with a photon in the heralded channel.

This heralding ratio is calculated in a very small sample of the doubly resonant 


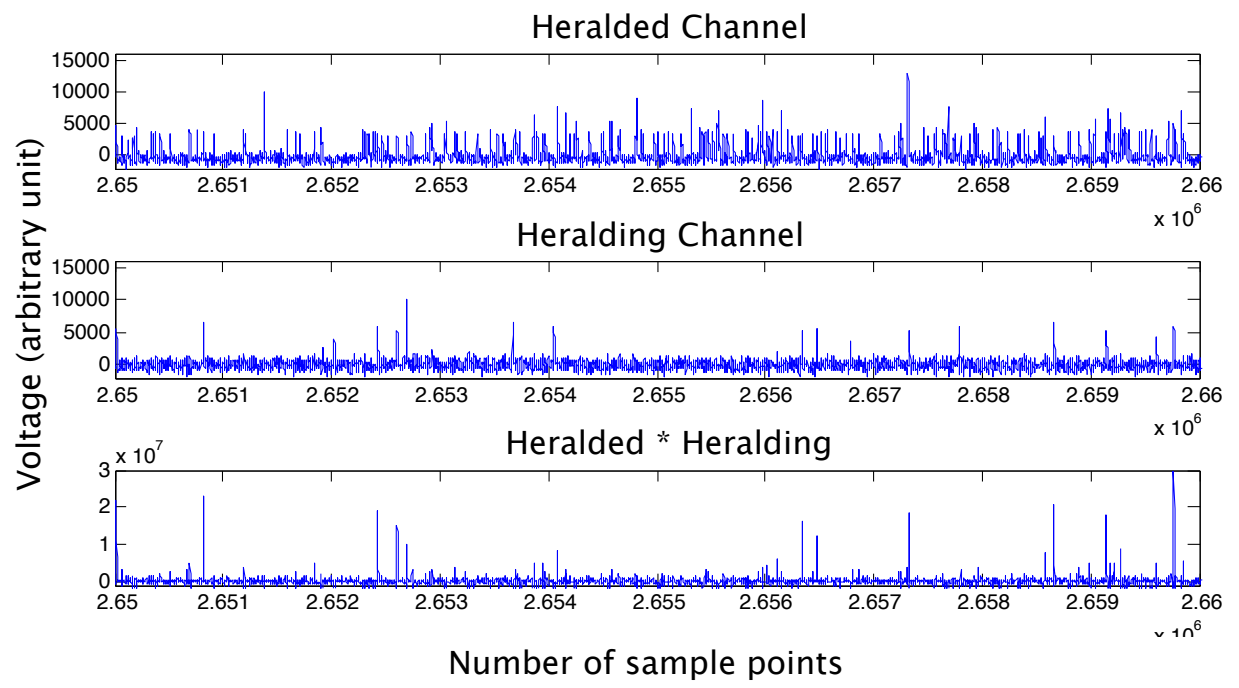

Figure 2.35: TES raw data on the heralding and heralded channels, zoomed in.

TES data. We then tried to go through the whole data and calculated the heralding ratio for larger samples. One should note that in this experiment we cannot lock the OPO in the well below oscillation threshold regime, so we tried to manually tune the piezo voltage by hand to stabilize the cavity length on the doubly resonance with few $\mathrm{nm}$ accuracy, as the minimum distance between doubly resonant modes in a cluster is about $2.2 \mathrm{~nm}$.

Despite careful tuning of the cavity length, we could not stay at doubly resonant modes for long time, therefore we divided the whole data points into shorter segments to make sure there is pure doubly resonant mode in each time interval. We then calculated the number of photons on the heralding and heralded channels and also the heralding ratio for each time interval Fig. 2.36.

This graph clearly shows two distinct regimes, on the left where the doubly reso- 


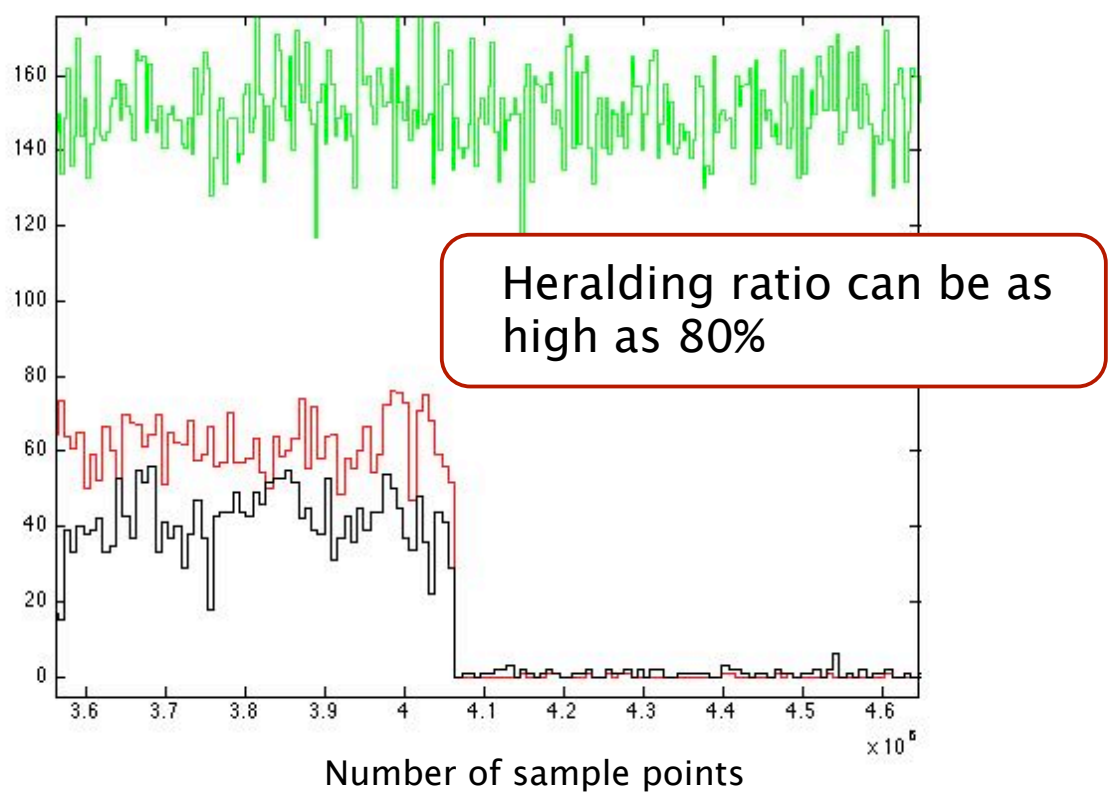

$$
\begin{aligned}
& \text { - Heralding ratio in } 3 \mathrm{~ms} \text { time interval } \\
& \text { - Number of heralding photons in } 3 \mathrm{~ms} \\
& \text { — Number of heralded photons in } 1.5 \mathrm{~ms}
\end{aligned}
$$

Figure 2.36: Number of heralding and heralded photons along with the heralding ratio on precise time intervals 
nant condition is fulfilled, then number of heralding photons and also the heralding ratio increases, and on the right where number of heralded photons decreases by a factor of 10. In both cases, the number of photons on the heralded channel is constant which totally supports our earlier discussions about the existence of singly and non resonant modes and the importance of appropriate filtering.

To calculate the heralding ratio we went through the TES raw data and looked for the coincident photon in the heralded channel upon detecting a photon in the heralding channel, we then calculated the ratio of coincident photon events to the heralded photon events as the heralding ratio.

The heralding ratio is not constant in all time interval but as soon as one hit doubly resonant modes, heralding ratio rises to higher than $50 \%$ and can go as high as $80 \%$. Most of these fluctuations is probably due to the difficulty of holding the OPO cavity on a doubly resonant mode .... by hand.

We also looked at the correlation function between the heralding and heralded channels Fig. 2.37, Figure 2.37.b shows correlation function calculated on doubly resonant modes, the correlation decays after 10 sample points which is equivalent to a photon peak length $(3 \mu s)$ and includes the photon rising and decaying times. So when two traces are delayed by a photon time then the correlation function approaches zero, which indicates that correlation is absolutely due to photon coincidences. Figure 2.37.c shows the correlation function calculated for the non resonant modes, it clearly shows that there does not exist any specific correlation between traces on non 
resonant part of the spectrum.

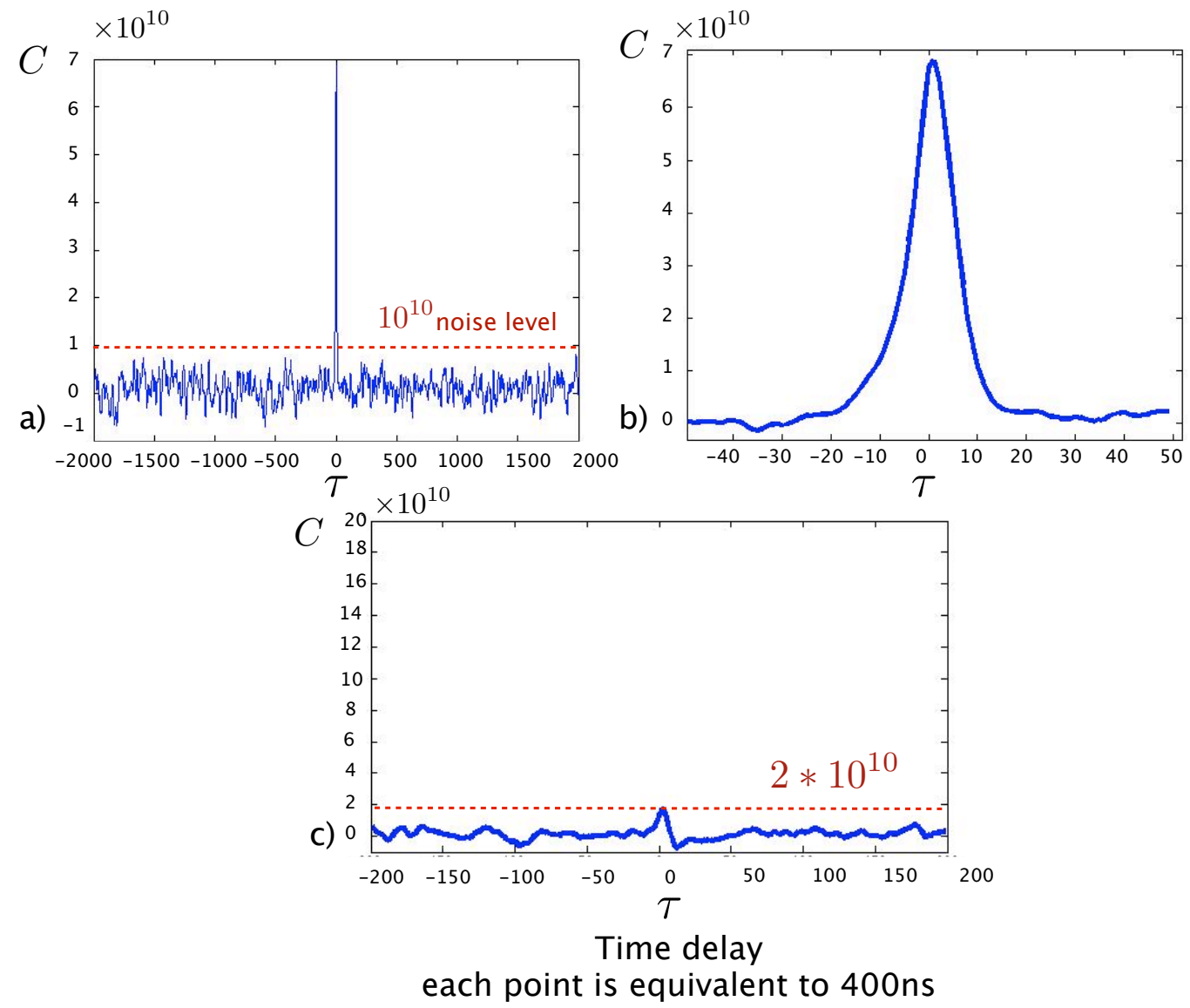

Figure 2.37: Correlation function: correlation $C(\tau)$ for a) doubly resonant modes, b) doubly resonant modes, zoomed in, c) singly and non resonant modes, the correlation for singly resonant modes is only $1 / 4$ of the doubly resonant peak but consistent with the noise level.

Achieving $80 \%$ heralding ratio is a record in our lab, as previously the heralding ratio could never go higher than $54 \%$.

It is worth mentioning that, other research groups have already reported singlephoton generation with high heralding ratios near $80 \%$, however each of these groups 
have different approaches and so advantageous and disadvantageous. Ramelow, et al [52] demonstrated the heralding of single-photons with $83 \%$ efficiency, they increased the heralding ratio by placing a multimode fiber on the heralded channel to collect whole SPDC emission including the multimode photons, multimode fiber on heralded channel increased the heralding ratio but it also results in heralding on multimode and broadband single photons, which are not necessary desirable for applications in quantum communication. Pereira, et al [53] and Smith et al [54] have also reported high heralding efficiencies up to $80 \%$, the heralding ratio is symmetric and singlemode in the first case, however both cases have corrected for detection inefficiencies and have not reported direct measurement of heralding ratio.

Here we only looked at single-photon heralding ratio. In fact, our slow data acquisition and processing speed results in low data sample rate and consequently not enough statistics on higher photon numbers, so we cannot calculate the heralding ratio for heralding on Fock states with arbitrary large photon numbers. However, we believe that increasing the speed of data acquisition will enable us to efficiently herald on Fock states with higher photon numbers.

\subsection{Conclusion}

In this chapter, we explained our efforts towards efficient generation of spatially and temporally well-defined Fock-states. We first showed that using SPDC generation on PPKTP or HGKTP crystals followed by a narrowband filtering cavity did not help us to efficiently herald for Fock states in narrow-band and single-mode quantum 
modes. We explained about the multimode nature of SPDC photons and how it prevents them from efficient collection into TES fibers, we also discussed about the huge loss in photon number correlation initiated from the Lorentzian lineshape of filtering cavity.

Eventually, we placed the nonlinear crystal inside a Fabry-Perot cavity, this time the cavity enhancement effect boosts the generation of SPDC modes in a well-defined cavity mode and allowed us to herald for narrow-band and single-mode Fock states. We calculated up to $80 \%$ heralding efficiencies for single-photon generation and we believed that this result can be generalized to higher photon number states using faster data acquision and data analysis systems. 


\section{Chapter 3}

\section{Fock state interferometry in the context of the information theory}

\subsection{Introduction}

Quantum mechanics imposes a fundamental limit on the estimation of an optical phase shift. The number-phase Heisenberg uncertainty between arms of the MZI interferometer, Eq. 1.65 will bound the phase precision to $\Delta \phi_{-}=\langle N\rangle^{-1}, \phi_{-}$being the optical phase difference between arms and $\langle N\rangle$ the average number of photons sent to the interferometer, as was discussed in section 1.3 .2 .

However, interferometers fed with classical light can never reach this fundamental limit of precision for phase estimation. In conventional interferometry, all photons are sent to one of the input arms, see section 1.3.2, and vacuum mode enters through the other arm, interference of light with vacuum mode results in a binomial probability law and yields the beamsplitter shot-noise limit of $\Delta \phi=\langle N\rangle^{-1 / 2}$, Eq. 1.66, therefore the interferometer's performance is degraded by the factor of $\langle N\rangle^{-1 / 2}$ with respect to the Heisenberg limit. 
In 1981, Caves showed that feeding the interferometer with a squeezed vacuum field can improve the interferometer precision to beat the shot noise limit [55]. Later, Yurke in 1986 [56] and also Holland and Burnett in 1993 [57] showed that how a Heisenberg-limited interferometer $\Delta \phi=\langle N\rangle^{-1}$ can be obtained using quantum light in correlated number states. Many theoretical and experimental studies were conducted and proved that a nonclassical light probe improves the precision in estimating the optical phase by the factor of $N^{-1 / 2},[6],[7],[8]$.

Nonclassical states of light are of great interest for ultraprecise optical phase estimation. Implementing quantum correlations has improved the field of interferometric sensing by predicting enhancement in the precision of optical phase measurements. Such enhancement has improved gravitational-wave interferometers [58], [59].

In this chapter, we study Heisenberg-Limited Interferometry (HLI) in context of the information theory. We show that the Heisenberg-limited phase estimation can improve resolution of optical phase discrimination, and have applications in the recently developed concept of the quantum reading of classical digital memory.

We propose a Fock State Interferometry (FSI) scheme inspired by the Holland and Burnett Interferometer for the problem of discriminating a finite number $M \geq 2$ of optical phase shifts. Our suggested interferometry scheme, which is a Mach-Zehnder Interferometer (MZI) fed with nonclassical light prepared in Fock states, Fig. 3.1. realizes a physical model for error free discrimination between $\mathrm{M}=2,3$ optical phases. This model can also discriminate between tinier phase shifts beyond what is feasible 
by classical protocols.

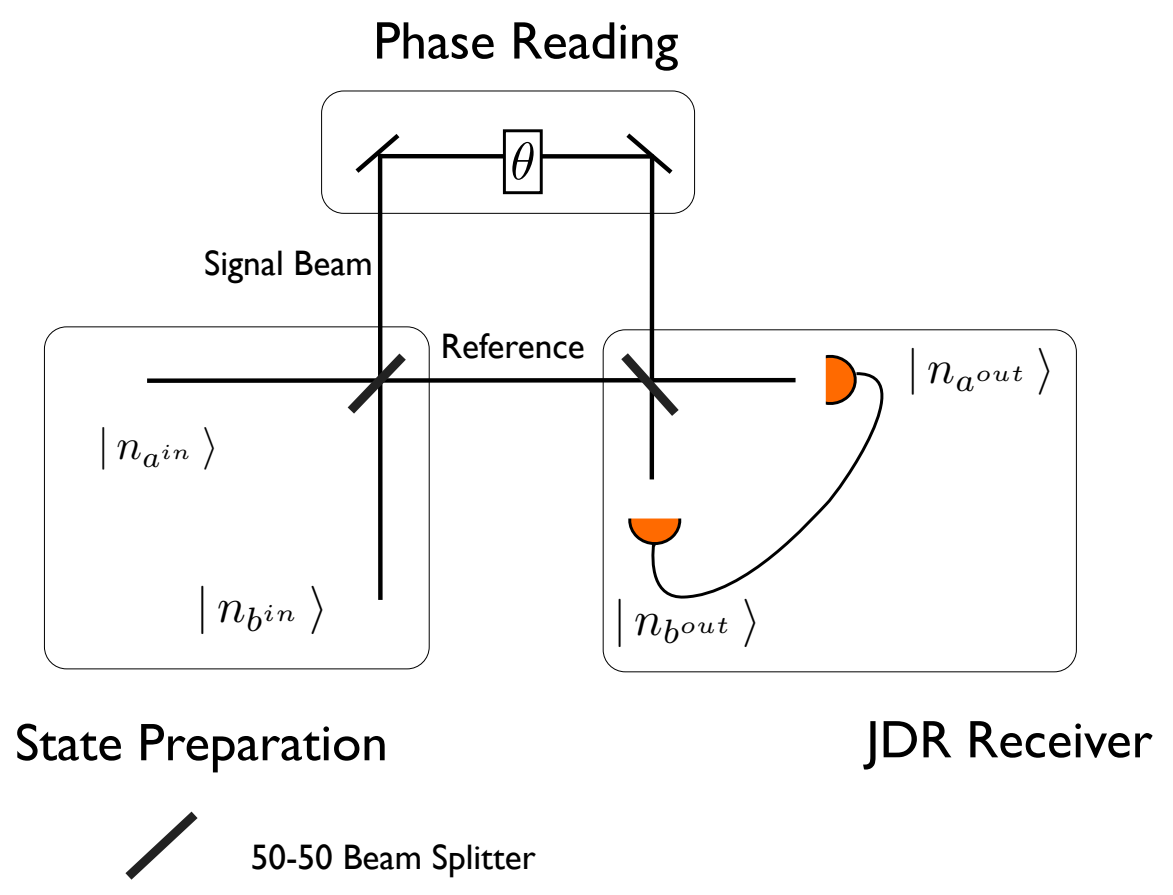

Figure 3.1: Phase estimation setup in FSI. To accurately estimate the phase applied on the phase reading unit, we first prepare the Fock state $\left|n_{a}\right\rangle\left|n_{b}\right\rangle$ then interfere it at the first 50-50 beam splitter of the MZI. One of the BS outputs, called signal will be sent to the phase reading unit and experience a phase shift and the other one will be used as the reference. Finally both the signal and reference beams will interfere on another 50-50 beam splitter and eventually they will be measured by Photon Number Resolving detectors (PNRs). Their photon number sum and differences will be used to estimate the phase $\theta$.

In optical communication terms, the ability to discriminate between M optical phase shifts can be beneficial for M-ary Phase Shift Keying (MPSK). MPSK is a digital modulation scheme that conveys M messages by modulating the optical phase of a probe signal. We show that using FSI we can accurately discriminate between $\mathrm{M},(\mathrm{M}=2,3)$ optical phase shifts. Moreover, we will show that FSI allows conveying the information encoded in smaller phase shifts. 
This Chapter is organized as follows, we first study the problem of discriminating the finite number $M \geq 2$ of optical phase shifts for M-ary BPSK and show that our model can discriminate between two or three phases with zero probability of error using only few photons, outperforming phase discrimination using coherent states with heterodyne and homodyne receivers.

In the second part, we study the quantum optical reading of classical digital memory and its applications in the technology of digital memories such as optical disks. We show that nonclassical light, along with a joint detection receiver, can outperform optical reading techniques in conventional optical disks and drivers. Optical reading in standard CD or DVD drives uses a laser-light probe and a direct-detection receiver and cannot read any more than 0.5 bit of information per transmitted photon. In contrast, our experimentally feasible model for optical reading can read 1 bit of information per transmitted photon. We will also show that our model is able to discriminate between smaller phases and is sensitive to smaller phase shifts in the memory encodings.

We also study denser optical encoding by implementing three distinct phases in each pixel and show that it is possible to read $\log _{2}(3) \sim 1.6$ bits of information per pixel using as few as two photons.

\subsection{Holland-Burnett Interferometer}

In 1993 Murray Holland and Keith Burnett [57] proposed a Heisenberg-limited quantum interferometer in which indistinguishable twin Fock states are simultaneously fed 
into input ports of a 50:50 beam splitter of a Mach-Zehnder interferometer (Fig. 3.1). The behavior of Holland and Burnett interferometer (HBI) in the low photon-number limit is predicted to be significantly different from that of a conventional MZI with shot noise limited phase sensitivity, Eq. (1.66).

Injecting indistinguishable twin-Fock states prepared in Eq. (3.1) to both input ports of the MZI interferometer enables one to estimate an optical phase shift difference $\Delta \phi_{-}$with sub-shot-noise precision.

$$
|\psi\rangle=\left|\hat{k}_{a}, \omega, \hat{\epsilon} ; n\right\rangle \otimes\left|\hat{k}_{b}, \omega, \hat{\epsilon} ; n\right\rangle
$$

$\hat{k}$ is the unit wave vector, $\omega$ the frequency, $\hat{\epsilon}$ the unit polarization vector, and $n$ the photon number.

The physics behind this interferometer and the beam splitter is similar to the Houng-Ou-Mandel (HOM) interference of the twin Fock states which was explained earlier in section 1.3.2. It employs photons prepared in states with maximum phase difference fluctuations $\left(\Delta \phi_{-}\right)$before the beam splitter and therefore minimum $\left(\Delta \phi_{-}\right)$ after the BS and on the phase reading block of Fig. 3.1. Study of the twin Fock states with higher photon numbers on the BS requires more complicated calculations compare to the simple HOM and is better to be treated in the Schwinger representation, which allows the application of angular momentum algebra and rotation matrices [9]. 


\subsubsection{The Schwinger Representation}

The Schwinger representation [60] introduces a mathematical description of passive lossless four-port optical devices based on rotations in an abstract 3D space. The application of the Schwinger representation to the analysis of optical interferometers was first demonstrated by Yurke et al [56].

Any linear passive lossless optical device with two input and two output ports can be described by a $2 \times 2$ unitary matrix of the special unitary group $\mathrm{SU}(2)$

$$
U=\left(\begin{array}{cc}
\cos \frac{\beta}{2} e^{i(\alpha+\gamma) / 2} & \sin \frac{\beta}{2} e^{i(\alpha-\gamma) / 2} \\
-\sin \frac{\beta}{2} e^{-i(\alpha+\gamma) / 2} & \cos \frac{\beta}{2} e^{-i(\alpha+\gamma) / 2}
\end{array}\right)
$$

Where $\alpha, \beta$ and $\gamma$ are Euler angles [61].

$\mathrm{U}$ operates on the two dimensional vector $(a, b)^{T}$, whose components $a$ and $b$ are the annihilation operators for the two input fields at each port of the system.

The homomorphism from $\mathrm{SU}(2)$ to the rotation group in three dimensions, $\mathrm{SO}(3)$, allows us to visualize the action of two-mode optical devices, such as beam splitters and phase shifters, as rotations in 3D space. The general rotation in Eq. (3.2) is mathematically equivalent to the rotation of the the following tridimensional vector $\vec{J}$ in 3D space:

$$
J=\left(\begin{array}{c}
J_{x} \\
J_{y} \\
J_{z}
\end{array}\right)=\frac{1}{2}\left(\begin{array}{c}
a^{\dagger} b+b^{\dagger} a \\
-i\left(a^{\dagger} b-b^{\dagger} a\right) \\
a^{\dagger} a-b^{\dagger} b
\end{array}\right)
$$

$J_{x}, J_{y}$ and $J_{z}$ follow the canonical commutation relations for quantum angular mo- 
mentum operators Eq. 3.4 , so $\vec{J}$ can be deemed a quantum angular momentum operator.

$$
\left[J_{i}, J_{j}\right]=i \hbar \epsilon_{i j k} J_{k}
$$

where $i, j, k \in\{x, y, z\}$, and $\epsilon$ is a Levi-Civita symbol.

The magnitude of the angular momentum $J^{2}$, can be calculated as:

$$
J^{2}=J_{x}^{2}+J_{y}^{2}+J_{z}^{2}=\frac{a^{\dagger} a+b^{\dagger} b}{2}\left(\frac{a^{\dagger} a+b^{\dagger} b}{2}+1\right)=\frac{N}{2}\left(\frac{N}{2}+1\right)
$$

where

$$
N=N_{a}+N_{b}=a^{\dagger} a+b^{\dagger} b
$$

is the total photon number operator.

Fock states $\left|n_{a}\right\rangle\left|n_{b}\right\rangle$ are the eigenstates of photon number operators $N_{a}$ and $N_{b}$ with eigenvalues $n_{a}$ and $n_{b}$. They are also eigenstates of $J$ and $J_{z}$, with respective eigenvalues $j(j+1)$ and $\mu$ :

$$
|j, \mu\rangle=\left|n_{a}, n_{b}\right\rangle
$$

with

$$
\begin{aligned}
& j=\frac{n_{a}+n_{b}}{2}=\frac{n}{2} \\
& \mu=\frac{n_{a}-n_{b}}{2}
\end{aligned}
$$

Therefore, Fock states can be treated as effective spin eigenstates with total spin $j$ proportional to their total photon number $n$ and $\mu$ proportional to their photon number difference, and any unitary operation can be viewed as rotation of this spin 
in the tridimensional space.

As an example, input state of the interferometer with $2 n$ photons in mode $a$ and vacuum in mode $b$ is identical to:

$$
|2 n\rangle_{a}|0\rangle_{b}=|j, j\rangle_{z} \quad, \quad j=n
$$

And the twin Fock state input which is required for Holland-Burnett interferometry is,

$$
|n\rangle_{a}|n\rangle_{b}=|j, 0\rangle_{z} \quad, \quad j=n
$$

\section{Mach-Zehnder Interferometer in the Schwinger Representation}

Any unitary operation on the quantum fields $a$ and $b$ can be viewed as the $\mathrm{SO}(3)$ rotation of the corresponding spin $\vec{J}$, Eq. 3.3 . And any arbitrary rotation of the spin $\vec{J}$ can be carried out via three successive rotations, called the Euler rotations as:

$$
\begin{gathered}
J^{\text {out }}=e^{i \alpha J_{z}} e^{i \beta J_{y}} e^{i \gamma J_{z}} J^{i n} e^{-i \gamma J_{z}} e^{-i \beta J_{y}} e^{-i \alpha J_{z}} \\
|\psi\rangle_{\text {out }}=e^{i \alpha J_{z}} e^{i \beta J_{y}} e^{i \gamma J_{z}}|\psi\rangle_{\text {in }}
\end{gathered}
$$

respectively in the Heisenberg and Schrodinger pictures.

In the Schwinger representation this arbitrary tridimensional rotation of the effective spin $\vec{J}^{\text {in }}$ is equivalent to the Euler angle parametrization of the $\mathrm{SU}(2)$ rotation of the two modes $a$ and $b$ basis, Eq. (3.2] 60] .

The representing $\mathrm{SO}(3)$ matrix for the Euler rotation is : 


$$
\left(\begin{array}{c}
J_{x}^{\text {out }} \\
J_{y}^{\text {out }} \\
J_{z}^{\text {out }}
\end{array}\right)=\left(\begin{array}{ccc}
c_{\alpha} c_{\beta} c_{\gamma}-s_{\alpha} s_{\gamma} & -c_{\gamma} s_{\alpha}-c_{\alpha} c_{\beta} s_{\gamma} & c_{\alpha} s_{\beta} \\
c_{\alpha} s_{\gamma}+c_{\beta} c_{\gamma} s_{\alpha} & c_{\alpha} c_{\gamma}-c_{\beta} s_{\alpha} s_{\gamma} & s_{\alpha} s_{\beta} \\
-c_{\gamma} s_{\beta} & s_{\beta} s_{\gamma} & c_{\beta}
\end{array}\right)\left(\begin{array}{c}
J_{x}^{\text {in }} \\
J_{y}^{\text {in }} \\
J_{z}^{\text {in }}
\end{array}\right)
$$

where $c_{(\alpha / \beta, \gamma)}=\cos (\alpha / \beta, \gamma)$ and $s_{(\alpha / \beta, \gamma)}=\sin (\alpha / \beta, \gamma)$

In the following, we study the effect of beam splitters and phase shifters in the Schwinger representation as building blocks of the Mach-Zehnder interferometer.

\section{Beam Splitter in the Schwinger Representation}

As described earlier in section 1.3.1, the representing $S U(2)$ matrix for the beam splitter with Fresnel intensity coefficients $\rho=\cos \phi / 2$ and $\tau=\sin \phi / 2$ is:

$$
U_{B S}=\left(\begin{array}{cc}
\cos \frac{\phi}{2} & i \sin \frac{\phi}{2} \\
i \sin \frac{\phi}{2} & \cos \frac{\phi}{2}
\end{array}\right)
$$

It is equivalent to the general $S U(2)$ matrix in Eq. (3.2) with the Euler angles $(\alpha, \beta, \gamma)=(\pi / 2, \phi, \pi / 2)$. Placing these Euler angles in Eq. (3.14) results in corresponding $S O(3)$ matrix for the effective spin $J^{\text {in }}$ rotation :

$$
\left(\begin{array}{c}
J_{x}^{\text {out }} \\
J_{y}^{\text {out }} \\
J_{z}^{\text {out }}
\end{array}\right)=\left(\begin{array}{ccc}
1 & 0 & 0 \\
0 & \cos \phi & \sin \phi \\
0 & -\sin \phi & \cos \phi
\end{array}\right)\left(\begin{array}{c}
J_{x}^{\text {in }} \\
J_{y}^{\text {in }} \\
J_{z}^{\text {in }}
\end{array}\right)
$$

Therefore, the effect of the beam splitter on modes a and $b$ is a rotation of effective spin $\vec{J}$ by $(-\phi)$ around the $x$ axis. The special case of the $50 / 50$ beam splitter with 
$\rho=\tau=\frac{1}{\sqrt{2}}, \phi=\pi / 2$ corresponds to a $(-\pi / 2)$ rotation around the $x$ axis :

$$
\begin{gathered}
J^{\text {out }}=e^{i \pi / 2} J^{i n} e^{-i \pi / 2} \\
|\psi\rangle_{\text {out }}=e^{i \pi / 2}|\psi\rangle_{\text {out }}
\end{gathered}
$$

\section{Phase Shifter in the Schwinger Representation}

The Phase shift $\theta$ between arms of the interferometer acts as: Eq. (1.57),

$$
U_{\theta}=\left(\begin{array}{cc}
e^{i \theta / 2} & 0 \\
0 & e^{-i \theta / 2}
\end{array}\right)
$$

which corresponds to the general $\mathrm{SU}(2)$ matrix $E q$. (3.2) with $(\alpha+\gamma=\theta, \beta=0)$.

The comparable $\mathrm{SO}(3)$ rotation matrix is,

$$
\left(\begin{array}{l}
J_{x}^{\text {out }} \\
J_{y}^{\text {out }} \\
J_{z}^{\text {out }}
\end{array}\right)=\left(\begin{array}{ccc}
\cos \theta & -\sin \theta & 0 \\
\sin \theta & \cos \theta & 0 \\
0 & 0 & 1
\end{array}\right)\left(\begin{array}{c}
J_{x}^{\text {in }} \\
J_{y}^{\text {in }} \\
J_{z}^{\text {in }}
\end{array}\right)
$$

which is a rotation of effective spin around $z$ by $\theta$.

$$
\begin{gathered}
J^{\text {out }}=e^{i \theta / 2} J^{\text {in }} e^{-i \theta / 2} \\
|\psi\rangle_{\text {out }}=e^{i \theta / 2}|\psi\rangle_{\text {out }}
\end{gathered}
$$

\section{Mach-Zehnder Interferometer}

The Mach-Zehnder Interferometer (MZI) is consisted of two 50/50 beam splitters, and a phase shifter as was studied earlier in section 1.3.1. So, the effect of MZI is 
equivalent to a $(-\pi / 2)$ rotation around $x$ axis, a $\theta$ rotation around $z$, and another $\pi / 2$ rotation around $x$.

$$
\begin{array}{r}
J^{\text {out }}=e^{-i(\pi / 2) J_{x}} e^{i \theta J_{z}} e^{i(\pi / 2) J_{x}} J^{i n} e^{i(\pi / 2) J_{x}} e^{-i \theta J_{z}} e^{-i(\pi / 2) J_{x}} \\
\left|\psi_{\text {out }}\right\rangle=e^{-i(\pi / 2) J_{x}} e^{i \theta J_{z}} e^{i(\pi / 2) J_{x}}\left|\psi_{\text {in }}\right\rangle=e^{i \theta J_{y}}\left|\psi_{\text {in }}\right\rangle
\end{array}
$$

Therefore the effect of MZI is equivalent to a single rotation of effective spin by $\theta$ around the $y$ axis.

The corresponding $\mathrm{SO}(3)$ matrix can be reconstructed by multiplying the $\mathrm{SO}(3)$ matrices for beam splitter, phase shifter and another beam splitter and will be equivalent to Eq. (3.14), with $(\alpha=\gamma=0, \beta=-\theta / 2)$,

$$
\left(\begin{array}{l}
J_{x}^{\text {out }} \\
J_{y}^{\text {out }} \\
J_{z}^{\text {out }}
\end{array}\right)=\left(\begin{array}{ccc}
\cos \theta & 0 & -\sin \theta \\
0 & 1 & 0 \\
\sin \theta & 0 & \cos \theta
\end{array}\right)\left(\begin{array}{c}
J_{x}^{\text {in }} \\
J_{y}^{\text {in }} \\
J_{z}^{\text {in }}
\end{array}\right)
$$

Here we are interested on the effect of MZI on Fock states, the eigenstates of effective spin $\vec{J},|j, \mu\rangle$. The probability function $P\left(\mu^{\prime}, \mu \mid \theta, j\right)$ for the input spin $|j, \mu\rangle$ to be measured after the interferometer as $\left|j, \mu^{\prime}\right\rangle$ for fixed $\theta$ and $J$ (the total photon number) can be described as a rotation matrix, which is a square matrix of dimension 
$2 j+1$ with general element,

$$
\begin{aligned}
P\left(\mu^{\prime}, \mu \mid \theta, j\right) & =\left|\left\langle j, \mu^{\prime} \mid \psi_{\text {out }}\right\rangle\right|^{2} \\
& =\left|\left\langle j, \mu^{\prime}\left|e^{i \theta J_{y}}\right| j \mu\right\rangle_{z}\right|^{2} \\
& =\left(d_{\mu^{\prime}, \mu}^{j}(\theta)\right)^{2}
\end{aligned}
$$

Rotation matrix elements, can be calculated as a function of Jacobi polynomials,

$$
d_{\mu^{\prime}, \mu}^{j}(\theta)=\left[\frac{(j+\mu) !(j-\mu) !}{\left(j+\mu^{\prime}\right) !\left(j-\mu^{\prime}\right) !}\right]^{1 / 2}\left(\sin \frac{\beta}{2}\right)^{\mu-\mu^{\prime}}\left(\cos \frac{\beta}{2}\right)^{\mu+\mu^{\prime}} \times P_{j-\mu}^{\left(\mu-\mu^{\prime}, \mu+\mu^{\prime}\right)}(\cos \beta)
$$

Mathematica program can calculate Jacobi polynomials and so rotation matrix elements for any arbitrary $j,\left(\mu, \mu^{\prime}\right) \leq j$.

\subsubsection{Measurement method}

The expectation value of the output intensity difference $\left\langle n_{a}-n_{b}\right\rangle_{\text {out }}$ of MZI with twin-Fock states as input modes, is independent of the phase shift $\theta$ between arms,

$$
\left\langle n_{a}-n_{b}\right\rangle_{\text {out }}=2\left\langle J_{z}\right\rangle_{\text {out }}=2\left\langle j, 0\left|\sin \theta J_{x}+\cos _{\theta} J_{z}\right| j, 0\right\rangle=0
$$

Therefore, average intensity difference contains no information about the phase and cannot be used to measure an optical phase shift. To overcome this difficulty, Holland and Burnett employed a Bayesian detection method [62] which consists in burst measurements of the output intensities, rather than average measurements. Later 
Kim et al [9] studied the HBI behavior and the Bayesian detection method in the Schwinger representation. The Bayesian detection method is based on the Baye's theorem: where one performs $p$ statistically independent measurements of $\mu$, at $j$ with predefined phase $\theta$, and can calculate the probability distribution of $\theta$ as,

$$
P\left(\theta, \mu_{1} \text { and } \ldots \ldots \ldots \ldots \text { and } \mu_{p}\right)=\prod_{i=1}^{p} P\left(\mu_{i} \mid \theta, j\right)=\prod_{i=1}^{p} d_{\mu, 0}^{j}(\theta)^{2}
$$

So far, we explained the Holland-Burnet interferometry scheme. At this point, we will consider similar setup, the MZI fed with twin Fock states, but with different measurement method, which relies on the single measurement outcome and is used for discriminating between predefined optical phases.

\subsection{MZI for phase discrimination}

We now consider the MZI for optical phase discrimination, under constraint on the total energy (photon number). We rely on the single measurement of $N_{a}-N_{b}$ or equivalently the $J_{z}$ measurement of the effective spin at the output ports of the interferometer, we then try to estimate the phase shift based on the single measurement outcome.

For simplicity, we first explain the concept with $M=2$ phases and then generalize it to higher values of $M$. We consider an unknown phase $\theta$ of MZI, which can take one of $M=2$ values, denoted $p_{1}$ and $p_{2}$ and define the estimated phase $\hat{\theta}$. The diagram in Fig. 3.2 shows all four possible scenarios that can happen during the phase estimation process. There is a possibility of success assigned with having $\theta=p_{1}, p_{2}$ 
and estimating $\hat{\theta}=p_{1}, p_{2}$.

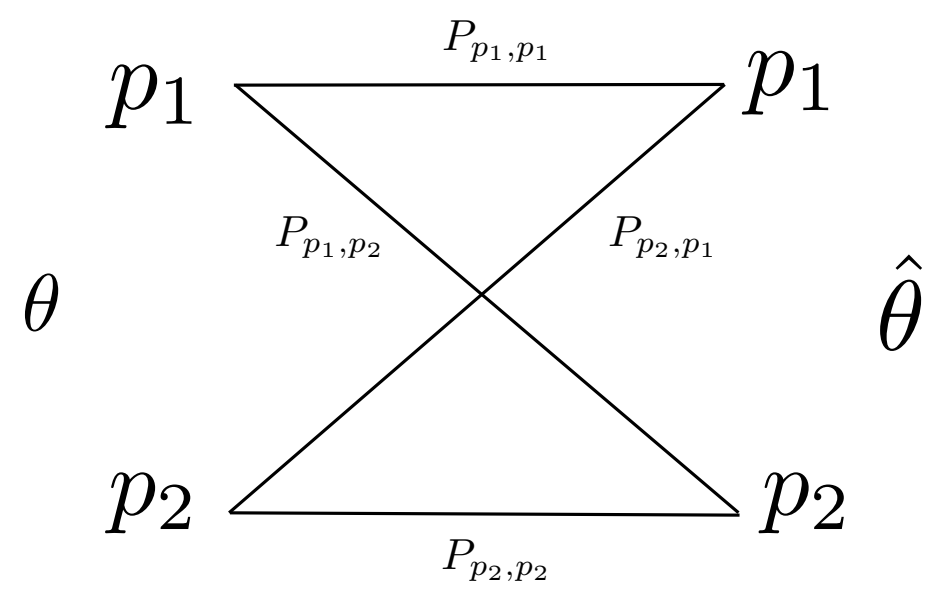

Figure 3.2: The four possible scenarios of the binary phase estimation process. The probabilities of correct estimation are $P_{p_{1}, p_{1}}$ and $P_{p_{2}, p_{2}}$, The probabilities of erroneous estimation are $P_{p_{1}, p_{2}}$ and $P_{p_{2}, p_{1}}$

A natural criterion to measure interferometer performance in the phase discrimination problem is the Probability of error $(\mathrm{Pe})$.

For example in the Fig. 3.2 the Probability of error is proportional to $P_{p_{1}, p_{2}}$ and $P_{p_{2}, p_{1}}$ vs the probability of success which depends on $P_{p_{1}, p_{1}}$ and $P_{p_{2}, p_{2}}$.

\section{Probability of Error, Pe}

The average error probability is defined as,

$$
P e=\sum_{i, j \neq i} p\left(\theta_{i}\right) P\left(\hat{\theta}_{j} \mid \theta_{i}\right)
$$

The way to extract the probabilities of error and success from probability distributions in Eq. (3.26), is to adopt specific selection rule. 


\section{Selection rule}

As mentioned earlier, for phase measurement we rely on the single $J_{z}$ measurement of the effective spin at the output ports and depending on the measurement outcome $\mu^{\prime}$ - one of the $2 j+1$ possible outcomes of the $J_{z}$ measurement- decide about the optical phase shift. The adopted selection rule is based on the maximum likelihood and suggest we examine both phases $p_{1}$ and $p_{2}$ for each measurement outcome and pick the one which is more likely to result in this specific outcome $\mu^{\prime}$ as the measured optical phase shift $\hat{\theta}$.

The Fig. 3.3 displays a probability chart to explain our selection rule. We have listed all possible measurement outcomes $\mu^{\prime}$ in the horizontal axis and phases in the vertical axis. Each element of this chart represents the probability of having phase $\theta=p_{1}\left(p_{2}\right)$ and measuring $\mu^{\prime}(-j, . ., 0, . ., j)$.

For each measurement outcome, we look at both phases $p_{1}$ and $p_{2}$ and the probability $P\left(\mu^{\prime}, \mu \mid p_{1(2)}, j\right)$ to rotate the initial state $|j, \mu\rangle$ to the measured state $\left|j, \mu^{\prime}\right\rangle$. We then pick the phase which has the greater probability (selection rule):

$$
\text { If } \begin{aligned}
P\left(\mu^{\prime}, \mu \mid p_{1}\right) \geq P\left(\mu^{\prime}, \mu \mid p_{2}\right) \longrightarrow \hat{\theta} & =p_{1} \\
\text { else } & \hat{\theta}=p_{2}
\end{aligned}
$$

Therefore, probabilities of success and error are directly proportional to elements of the rotation matrix Eq. (3.26). For example, in the chart in Fig. 3.3, the success 


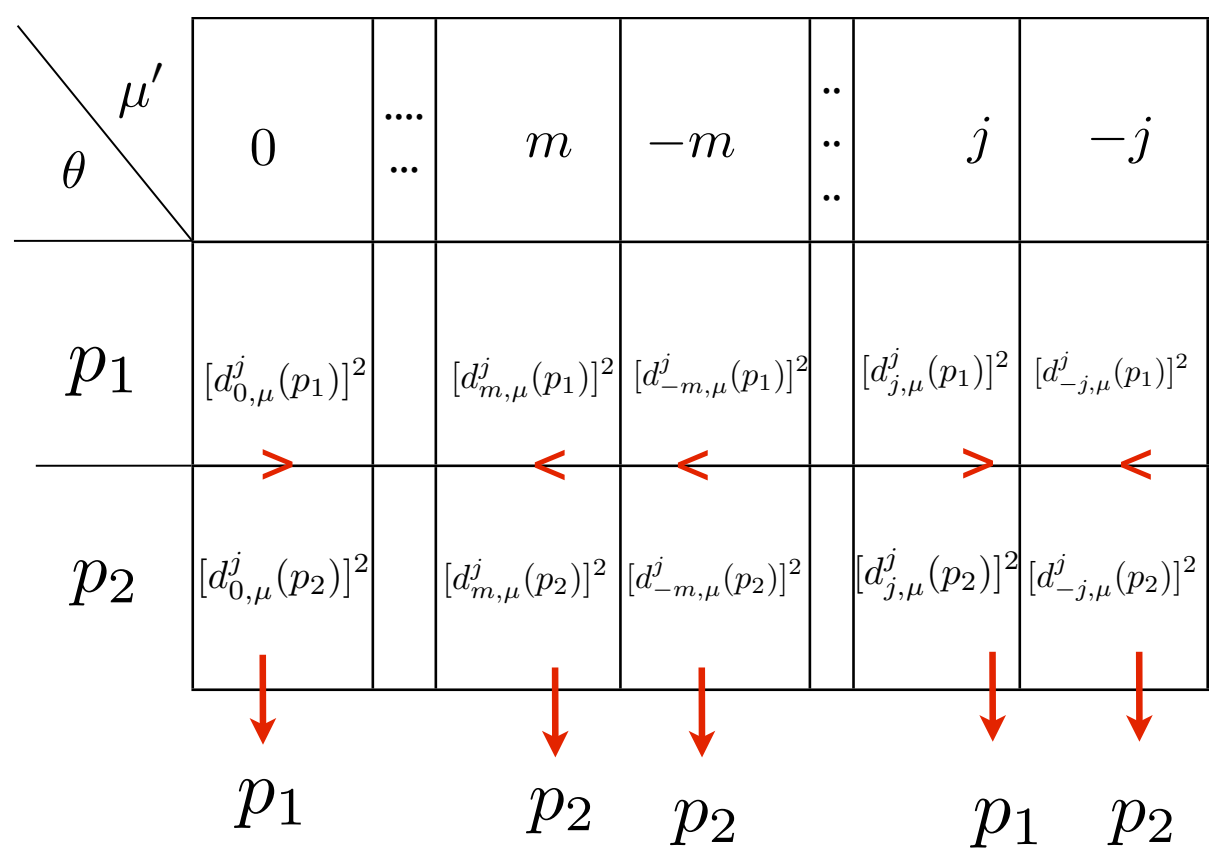

Figure 3.3: An example of a selection rule chart for specified probability distribution with effective input spin $|j, \mu\rangle$. The horizontal axis, $\mu^{\prime}$ indicates all possible $2 j+1$, $J_{z}$ measurement outcomes, and the vertical presents predefined phase shifts. Each element is the probability that the corresponding phase rotate the $|j, \mu\rangle$ to $\left|j, \mu^{\prime}\right\rangle$. For each $\mu^{\prime}$, we compare two probabilities $d_{\mu^{\prime}, \mu}^{j}(p)$ for $p_{1}$ and $p_{2}$ as indicated with red signs and depending on their values pick one of the phases as measurement result $\hat{\theta}$. Constructing this chart and examining all elements allows us to calculate all probabilities of error and success. 
probability of applying $p_{1}$ and measuring $p_{1}$ is equal to:

$$
P_{p_{1}, p_{1}}=\left[d_{0, \mu}^{j}\left(p_{1}\right)\right]^{2}+\left[d_{j, \mu}^{j}\left(p_{1}\right)\right]^{2}+\ldots
$$

But the failure probability of applying $p_{1}$ and measuring $p_{2}$ is:

$$
P_{p_{1}, p_{2}} \propto\left[d_{m, \mu}^{j}\left(p_{1}\right)\right]^{2}+\left[d_{-m, \mu}^{j}\left(p_{1}\right)+d_{-j, \mu}^{j}\left(p_{1}\right)\right]^{2}+\ldots \ldots
$$

Since we are relying only on one set of measurement outcomes, then the probability of error can be very high. Therefore, in this work we carefully examine all combinations of phases and pick the $p_{1} \mathrm{~s}$ and $p_{2} \mathrm{~S}$ which result in least probability of error and are most suitable for optical phase discrimination problem. It is clear from the selection rule chart that set of phases that have probability of one in one element and the zero in all other corresponding horizontal elements results in error-free phase discrimination. Any of these phases rotates the input eigenstate $|j, \mu\rangle$ to another eigenstate $|j, k\rangle$ with $d_{\mu, \mu^{\prime}}^{j}(p)=1(0)$ for $\mu^{\prime}=k\left(\mu^{\prime} \neq k\right)$. Zero phase rotation is equivalent to the identity operator and leaves the input eigenstate unchanged, therefore zero phase is always one of the optimal phases which can minimize the probability of error. Therefore, we fix $p_{1}=0$ and only vary $p_{2}=p$.

\section{Mathematica programs}

We wrote a Mathematica program to model the effect of MZI on any arbitrary spin eigenstate $|j, \mu\rangle$ with randomly large $j$. The program calculates all probability distributions in Fig. 3.2 for whole set of phases, $p_{1}$ and $p_{2},\left(0<p_{1}, p_{2}<2 \pi\right)$. The 
Mathematica program presented in Fig. 3.4 is an example of such program which models effective spin $\left|j, m_{1}\right\rangle=|2,1\rangle$. It is consist of three "For" loops, two of them go through all possible values of $p_{1}$ and $p_{2}$ between zero and $2 \pi$ and the third loop is on all $2 j+1$ values of $\mu^{\prime}$. For each fixed value of $p_{1}, p_{2}$ and $J_{z}$ measurement outcomes $\mu^{\prime}=m_{2}$, program compares two probabilities of $P\left(m_{2}, m_{1} \mid p_{1}\right)$ and $P\left(m_{2}, m_{1} \mid p_{2}\right)$ :

If $P\left(m_{2}, m_{1} \mid p_{1}\right) \geq P\left(m_{2}, m_{1} \mid p_{2}\right)$, then the estimated phase is $\hat{\theta}=p_{1}$, which means every time that we measure $m_{2}$ we call $\hat{\theta}=p_{1}$. It is correct guess with the probability of $P\left(m_{2}, m_{1} \mid p_{1}\right)$ and with $P\left(m_{2}, m_{1} \mid p_{2}\right)$ our guess is a failure and cause an error in phase discrimination. These probabilities respectively add up to the probabilities of success and failure as, $P_{p 1, p 1} \rightarrow P_{p 1, p 1}+P\left(m_{2}, m_{1} \mid p_{1}\right)$ and $P_{p 2, p 1} \rightarrow P_{p 2, p 1}+P\left(m_{2}, m_{1} \mid p_{2}\right)$. Eventually, this program loop through all possible $J_{z}$ measurement outcomes and calculates probabilities of $P_{p 1, p 1}, P_{p 1, p 2}, P_{p 2, p 1}, P_{p 2, p 2}$ and Pe, for all sets of phases. We then compare the action of phase discrimination setup for all phases $p_{1}, p_{2}$ and pick the ones which have best performance (least Pe).

Note that, this program does not only consider the effect of MZI on twin-Fock state which is preferred for HBI, but is very general and can calculate the behavior of MZI with arbitrary input state:

$$
|2 n-m\rangle_{a}|m\rangle_{b}=|j, m\rangle_{z} \quad n=j
$$




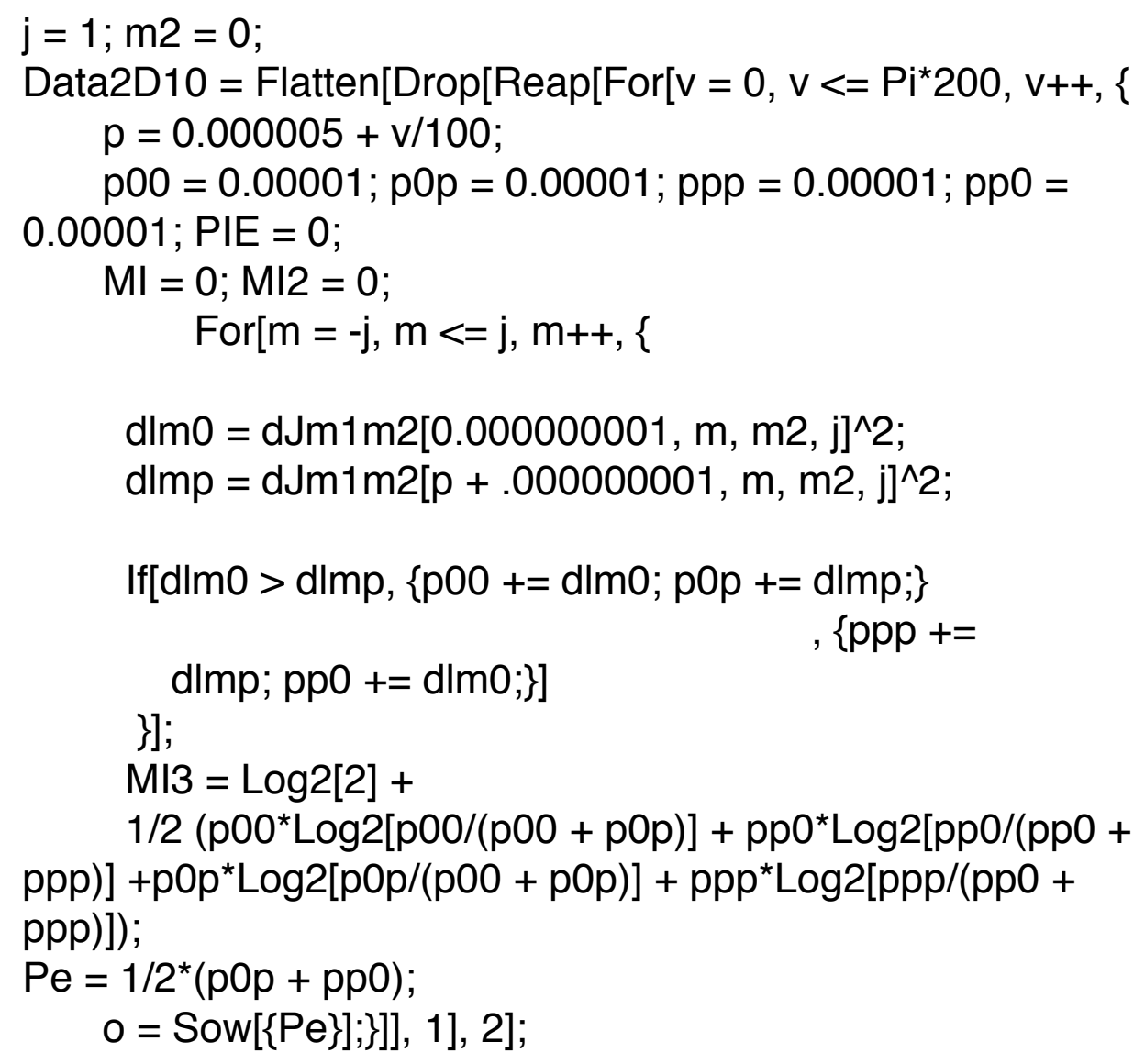

Figure 3.4: The Mathematica program to calculate the effect of MZI on effective spin $\left|j, m_{1}\right\rangle$ of input photons. It calculates probabilities of error and success for two phase discrimination on all possible phase combinations $P_{1}, P_{2}$. 


\subsection{Information theory}

In this section we briefly explain some basic concepts of the information theory. We will use these concepts later to study the behavior of FSI, then compare FSI with the more general case of a MZI with various types of input quantum states.

For any probability distribution, we recall the definition of Shannon entropy. Let X be a discrete random variable with the probability density function, $p(x), x \in X$. The Shanon entropy of the random variable $X$ is :

$$
H(X)=-\sum_{x \in X} p(x) \log _{2} p(x)
$$

The unit of entropy is bit. The entropy is a measure of the information required on average to describe the probability distribution $X$. For example the fair coin toss results in two outcome, each with equal probability $\frac{1}{2}$,

$$
\begin{gathered}
X=\left\{\frac{1}{2}, \frac{1}{2}\right\} \\
H(X)=-\frac{1}{2} \log _{2}\left(\frac{1}{2}\right)-\frac{1}{2} \log _{2}\left(\frac{1}{2}\right)=1
\end{gathered}
$$

So, it contains 1 bit of information, in other words, 1 bit of information is required to describe the $X$ distribution.

\section{Conditional entropy}

We now extend the concept of the entropy of a single random variable to a pair of random variables $X, Y$. The conditional entropy is the expected value of the entropies 
of the conditional distributions, averaged over the conditioning random variable:

$$
\begin{aligned}
H(Y \mid X) & =\sum_{x \in X} p(x) H(Y \mid X=x) \\
& =-\sum_{x \in X} p(x) \sum_{y \in Y} p(y \mid x) \log _{2} p(y \mid x)
\end{aligned}
$$

where $p(y \mid x)$ is the conditional probability of measuring $y$ given that $x$ occurred.

\section{Mutual Information}

We also introduce the mutual information (MI), which is a measure of the amount of information that one random variable $X$ contains about another random variable $Y$. It is equivalent to the reduction in the uncertainty of one random variable due to the knowledge of the other. For random variables $X$ and $Y$ with probability functions $p(x), x \in X$ and $p(y), y \in Y$ and the conditional entropies $H(X \mid Y)$ and $H(Y \mid X)$, the mutual information can be written as:

$$
I(X ; Y)=H(X)-H(X \mid Y)=H(Y)-H(Y \mid X)
$$

One way to study the general problem of encoding the information in $\mathrm{M}$ optical phases, M-ary phase shift keying (PSK), or the more specified problem of optical reading is to look at the mutual information between the applied optical phases, $\theta$ and the measured phases $\hat{\theta}$. Ideally, $\hat{\theta}$ should contain all the information about $\theta$ and the mutual information $I(\theta ; \hat{\theta})$ should be equal to the amount of information in random variable $\theta, H(\theta)$.

Lets assume information is encoded in $M$ optical phases with equal a priori prob- 
abilities so,

$$
\begin{gathered}
\theta=\left\{\theta_{1}, \ldots, \theta_{i}, \ldots, \theta_{M}\right\} \\
P(\theta)=\left\{\frac{1}{M}, \ldots, \frac{1}{M}, \ldots, \frac{1}{M}\right\} \\
H(\theta)=\log _{2} M
\end{gathered}
$$

Then, one tries to estimate the optical phases $\hat{\theta}$, a random variable with the probability mass function ( Fig. 3.2):

$$
\begin{aligned}
\hat{\theta} & =\left\{\hat{\theta_{1}}, \ldots, \hat{\theta_{j}}, \ldots, \hat{\theta_{M}}\right\} \\
P(\hat{\theta}) & =\left\{P\left(\hat{\theta_{1}}\right), \ldots, P\left(\hat{\theta_{j}}\right), \ldots, P\left(\hat{\theta_{M}}\right)\right\}
\end{aligned}
$$

Note that $\hat{\theta}$ is not necessary the same random variable as $\theta$, but their similarity and the overlap in their information contents, defined as $I(\theta ; \hat{\theta})$, is a good measure of success in the phase encoding problem.

$I(\theta ; \hat{\theta})$ can be calculated as:

$$
\begin{aligned}
I(\theta ; \hat{\theta}) & =H(\theta)-H(\theta \mid \hat{\theta}) \\
& =H(\theta)-\sum_{j} P\left(\hat{\theta}_{j}\right) \sum_{i} p\left(\theta_{i} \mid \hat{\theta}_{j}\right) \log _{2} p\left(\theta_{j} \mid \hat{\theta}_{i}\right)
\end{aligned}
$$

$p\left(\hat{\theta}_{j} \mid \theta_{i}\right)$ is the probability that one can directly extract from experiment so, we employ 
the Bayes theorem:

$$
\begin{gathered}
p\left(\theta_{i} \mid \hat{\theta}_{j}\right)=\frac{p\left(\hat{\theta}_{j} \mid \theta_{i}\right) p\left(\theta_{i}\right)}{p\left(\hat{\theta}_{j}\right)} \\
p\left(\hat{\theta}_{j}\right)=\sum_{i} p\left(\hat{\theta}_{j} \mid \theta_{i}\right) p\left(\theta_{i}\right)
\end{gathered}
$$

and substitute $p\left(\theta_{i} \mid \hat{\theta}_{j}\right)$ with $p\left(\hat{\theta}_{j} \mid \theta_{i}\right)$ in $I(\theta ; \hat{\theta})$ :

$$
\begin{aligned}
I(\theta ; \hat{\theta}) & =H(\theta)-\sum_{i, j} P\left(\theta_{i}\right) \frac{p\left(\hat{\theta}_{j} \mid \theta_{i}\right) p\left(\theta_{i}\right)}{p\left(\hat{\theta}_{i}\right)} \log _{2} \frac{p\left(\hat{\theta}_{j} \mid \theta_{i}\right) p\left(\theta_{i}\right)}{p\left(\hat{\theta}_{i}\right)} \\
& =\sum_{i} p\left(\theta_{i}\right) \log _{2} p\left(\theta_{i}\right)-\sum_{i, j} p\left(\theta_{i}\right) p\left(\hat{\theta}_{j} \mid \theta_{i}\right) \log _{2} \frac{p\left(\hat{\theta}_{j} \mid \theta_{i}\right)}{\sum_{k} p\left(\hat{\theta}_{j} \mid \theta_{k}\right)} \\
& =\log _{2} M-\sum_{i, j} \frac{p\left(\hat{\theta}_{j} \mid \theta_{i}\right)}{M} \log _{2} \frac{p\left(\hat{\theta}_{j} \mid \theta_{i}\right)}{\sum_{k} p\left(\hat{\theta}_{j} \mid \theta_{k}\right)}
\end{aligned}
$$

$I(\theta ; \hat{\theta})_{\text {max }}=\log _{2} M$, when $P\left(\hat{\theta}_{j} \mid \theta_{i}\right)_{i=j}=1$ and $P\left(\hat{\theta}_{j} \mid \theta_{i}\right)_{i \neq j}=0$.

$P\left(\hat{\theta}_{j} \mid \theta_{i}\right)_{i \neq j}$ is the probability of having phase $\theta_{i}$ but estimating the wrong phase $\hat{\theta}_{j}$ which results in error, similar to the cross probabilities in Fig. 3.2 .

\subsection{Optical Phase Discrimination}

In this section we study the problem of discriminating a finite number $M \geq 2$ of optical phase shifts. We employ the Heisenberg limited Twin Fock state interferometry (TFSI) inspired by the Holland and Burnett proposal, as phase discrimination set up (Fig. 3.1), and compare its performance with the more general case of a MachZehnder Interferometer (MZI) fed with photons in various input Fock states, Fock State Interferometer (FSI). 
The TFSI is very well studied in the Schwinger representation by Kim et al, 9], they have considered TFSI for phase estimation and have shown that it is capable of phase estimation with the Heisenberg-limited resolution. In this chapter we study TFSI in the Schwinger representation but focus on the phase discrimination problem, which may be viewed in communication terms in analogy with M-ary phase-shift keying (PSK) 63.

The criteria which is typically used to measure the performance of an optical discrimination scheme is the probability of error Pe, Eq. 3.30. We show that TFSI or in general MZI with various Fock states as input (FSI) provides a feasible experimental scheme for optical phase discrimination with zero probability of error for $\mathrm{M}=2$ and very low error probabilities for $\mathrm{M}=3$. We also show that each of these experimental schemes are optimal in discriminating between specific set of phases $p_{1}, p_{2}$. Interestingly TFSI is able to discriminate between optical phase shifts separated by $\pi / 2$ which is in contrast to what the classical interferometry can do, it can only discriminate between phases separated by $\pi$.

In this section we study phase discrimination in detail for $M=2,3$ and then will discuss about higher values of $M$ and why their consideration is not beneficial in this interferometric scheme. We first consider $M=2$, it is equivalent to encoding information using two optical phases as Binary Phase-Shift Keyed (BPSK) modulation which is used in the current technology of CD's and DVD's. 


\subsubsection{Binary phase discrimonation, $\mathrm{M}=2$}

For the complete study of the binary phase discrimination, we consider a more general interferometry scheme, the MZI with the Fock state input in the generic form of $|2 n-\mu\rangle_{a}|\mu\rangle_{b}=|j, \mu\rangle_{z}$, Eq. (3.35), and compare its performance with the TFSI, which is a MZI fed with indistinguishable twin-Fock states $|n\rangle_{a}|n\rangle_{b}$. In the Schwinger representation, twin Fock state is equivalent to the effective spin $|j, 0\rangle_{z},(j=n)$. The special case of the effective spin, with $\mu=j$ is equivalent to the shot noise limited (SNL) interferometer, in which all photons are injected into only one input port of the interferometer and vacuum enters the other port. SNL interferometers is studied in detail in section 1.3.2.

In order to study the behavior of interferometer on different input states, we calculate the interferometer performance Pe and the mutual Information MI, for all $p$ phases. We then determine optimal phases depending on the specific choice of input state,

Fig. 3.5 shows the performance of the interferometer as a function of phase $\mathrm{p}$ when total of two photons are injected to the interferometer, photons can either be prepared in twin-Fock state $|1\rangle_{a}|1\rangle_{b}$ and fed into both ports of interferometer or they can be prepared in $|2\rangle_{a}|0\rangle_{b}$ and fed into only one arm. In both cases we can find an optimal phase $p_{i}$ for which $P e=0$. However, each of these cases have different optimal phases, the single-ported interferometer is optimal for discriminating between phases $(0, \pi)$, similar to the classical interferometers. But the interferometer fed with 


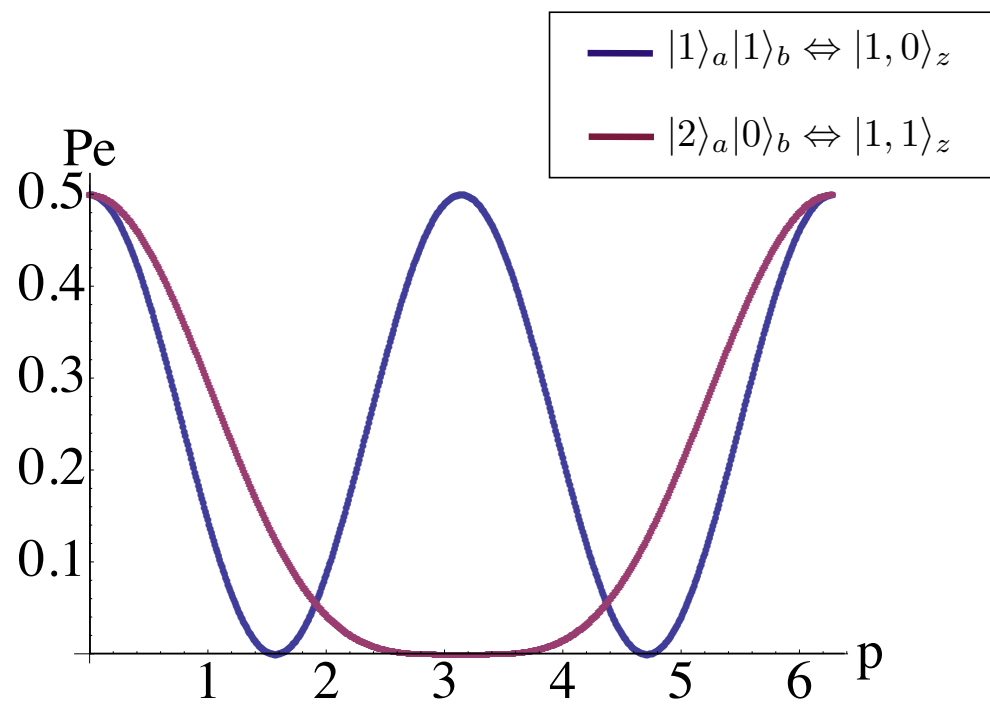

Figure 3.5: Probability of error Pe vs $p$ for optical phase discrimination between two phases $0, p$ and for two input states: pink) for effective spin $|1,1\rangle_{z}$ which is equivalent to interferometer fed with $|2\rangle_{a}|0\rangle_{b}$, two photons in one arm and vacuum enters the other arm. Blue) for effective spin $|1,0\rangle_{z}$ or equivalently interferometer fed with $|1\rangle_{a}|1\rangle_{b}$, twin-Fock photons into interferometer.

twin Fock state (TFSI) can discriminate between $\left(0, \frac{\pi}{2}\right)$. It suggest that TFSI is good for higher resolution optical phase discrimination and can have applications in higher resolution phase discrimination or denser optical encoding.

We also looked at the interferometer fed with higher photon numbers in average $\left(n_{\text {avg }}=2,3\right)$ in Fig. 3.6. It is clear from this diagram that our interferometry scheme is always adequate for error free 2-ary phase discrimination, however the optimum $\mathrm{p}$ value very much depends on the input state. The interferometer fed with twinFock state (TFSI) is optimum for discriminating between phases $(0, p=\pi / 2-\eta)$, in contrast, the single ported interferometer , $\mu=0$ is not ideal at this phase and its performance is optimum at phases $(0, \pi-\epsilon<p<\pi+\epsilon)$, both $\eta$ and $\epsilon$ are 

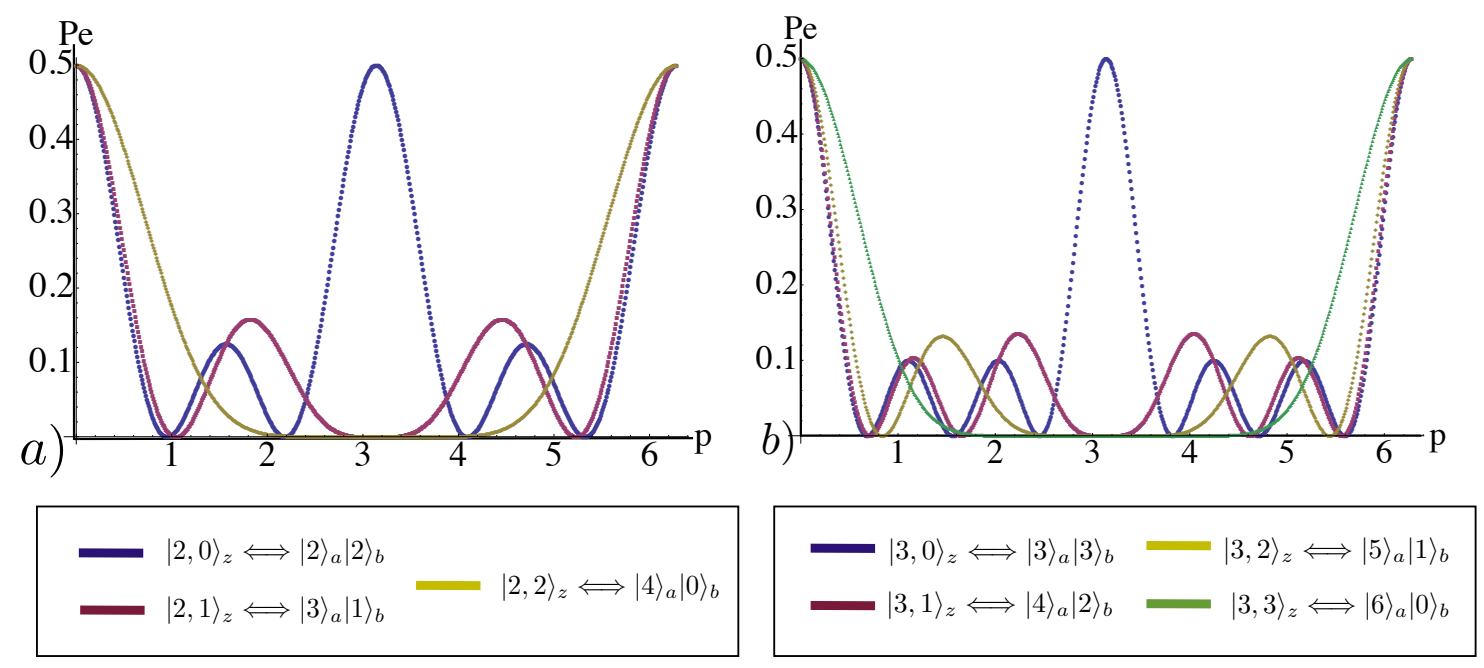

Figure 3.6: Probability of error (Pe) vs $p$ for optical phase discrimination between two phases $0, p$ and for various input states with constant average photon number a) $n_{\text {avg }}=2$. b) $n_{\text {avg }}=3$.

small numbers and their values increase by increasing the average photon number. Therefore, the single ported interferometer is not ideal for discriminating between phases separated by $\pi / 2$ or less. The act of the Interferometer fed with effective spin $|j, \mu\rangle,(0<\mu<j)$ is more interesting, it has two optimum $p$, one close to $\pi / 2$ and the other at $\pi$, this makes them an excellent candidate for 2-ary phase discrimination on phases separated both by $\frac{\pi}{2}$ and by $\pi$.

We have further studied the interferometer with higher average photon numbers then picked the optimum $\mathrm{p}$ for each $N_{\text {avg }}$ and plotted in Fig. 3.7. The optimum $\mathrm{p}$ always decreases by increasing the average photon number (higher spins), so sending more and more photons to the interferometer allows us to successfully discriminate between smaller and smaller phases.

In the following we compare the behavior of our interferometry scheme for 2-ary 


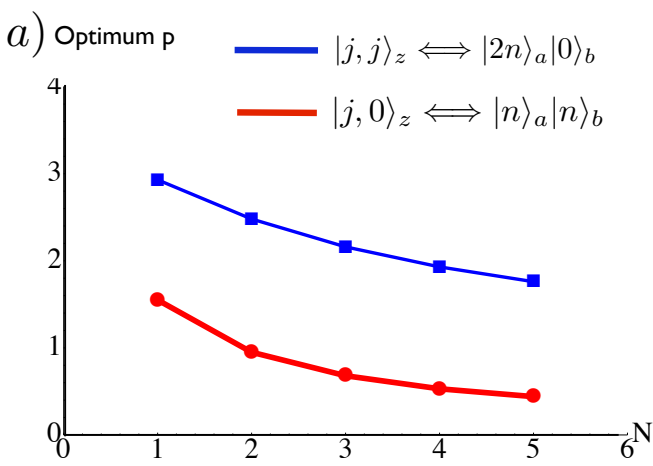

b)

\begin{tabular}{|c|c|c|c|c|}
\hline$n_{\text {avg }}$ & $\begin{array}{c}\mathrm{P} \\
\mathrm{HBI}\end{array}$ & $\begin{array}{c}\mathrm{P} \\
\text { Single ported }\end{array}$ & $\mathrm{MI}$ & $\mathrm{Pe}$ \\
\hline $\mathrm{I}$ & 1.56 & 2.93 & $\mathrm{I}$ & 0 \\
\hline 2 & 0.95 & 2.48 & $\mathrm{I}$ & 0 \\
\hline 3 & 0.68 & 2.16 & $\mathrm{I}$ & 0 \\
\hline 4 & 0.53 & 1.93 & $\mathrm{I}$ & 0 \\
\hline 5 & 0.44 & 1.76 & $\mathrm{I}$ & 0 \\
\hline
\end{tabular}

Figure 3.7: a) Optimum p vs $N_{\text {avg }}$, the value of optimum p decreases by increasing the average photon number. The optimum $\mathrm{p}$ is smaller for twin-Fock state input $|n\rangle_{a}|n\rangle_{b}, \forall n$. b) The optimum p (for both TFSI and single ported interferometers), Pe and the Mutual Information (MI), for each $N_{\text {avg }}$ listed in a chart.

phase discrimination with the classical interferometer fed with coherent states.

\section{2-ary phase discrimination using coherent states of light}

In binary phase-shift keyed encoding using coherent states, the phase modulated coherent states are given as $|\alpha\rangle$ and $|-\alpha\rangle$. In low photon number regime, these states have small amplitudes $\left(|\alpha|=\sqrt{N_{\text {avg }}}\right)$ and are largely overlapping (nonorthogonal), so the ability to successfully distinguish these states at the receiver is limited, the minimum error has completely analyzed and solved by Helstrom [64] and is known as the Helstrom bound. Researchers have proposed various detection schemes to approach this bound for coherent state discrimination. The simplest possible receiver which rely on Gaussian operations is the Homodyne receiver [65], the error probability for coherent state discriminating using Homodyne receiver is given by [66]: 


$$
\operatorname{Pe}(\alpha)=\frac{1}{2}\left(1-\operatorname{erf}\left(\frac{|\alpha|}{2}\right)\right)
$$

where $\operatorname{erf}$ is the error function defined as:

$$
\operatorname{erf}(x)=\frac{2}{\sqrt{\pi}} \int_{0}^{x} e^{-t^{2}} d t
$$

\section{Dolinar Receiver:}

In 1973 Dolinar proposed an adaptive measurement scheme that precisely achieves the Helstrom bound for discriminating between two pure coherent states. The Dolinar receiver is based on a combination of photon counting and real-time feedback control. The minimum probability of error using the Dolinar receiver is:

$$
P e_{\min }=\frac{1}{2}\left(1-\sqrt{1-e^{-4|\alpha|^{2}}}\right)
$$

This is the lowest possible error in distinguishing between two pure coherent states. Therefore, one cannot reach to the zero probability of error using low intensity coherent states. However, Nair et,.al, [67], showed that in low photon regime when $N_{\text {avg }} \geq \frac{M-1}{2}$, here $M=2$, there always exist an optimum quantum state which can perfectly distinguish between $\mathrm{M}$ optical phases. Their proposed quantum state is:

$$
|\psi\rangle_{s}=\frac{1}{M}\left(|0\rangle_{s}+\ldots \ldots \ldots+|M-1\rangle_{s}\right)
$$


Where, the sub $s$ indicates that, $\psi_{s}$ is a quantum state on the imaging probe (signal). Their proposed state is the so called Phase state, the eigenstate of Phase operator with minimum phase uncertainty, thus there is no surprise to act as the optimum state for phase discrimination. They also proposed that for $N_{a v g}<\frac{M-1}{2}$, a single-mode probe prepared in $|\psi\rangle_{s}=\sum_{\nu=0}^{M-1} \sqrt{p_{\nu}|\nu\rangle}, p_{\nu} \geq 0$ achieves the minimum error probability.

For $M=2$, the minimum error probability can be written as:

$$
P e=\frac{1}{2}-\sqrt{(N(1-N))}
$$

where $N=N_{a v g}$, the average photon number on the signal probe.

It is worth mentioning that, Nair, et al did not propose any experimental set up to generate the optimum state Eq. (3.52). On other hand, our proposed interferometer, provides a feasible experimental scheme for error free 2-ary phase discrimination, our scheme does not outperform the optimum state Eq. 3.52 proposed by them for energy efficient phase imaging but can discriminate phases with zero probability of error but by implementing few more photons. We also show that our measurement setup which is a MZI fed with Fock state input and with the specific joint detection receiver as was explained earlier in section 3.2.2, outperforms the performance of the interferometers with coherent state inputs and with Homodyne or Dolinar receivers Fig. 3.8 .

Classical interferometers can typically distinguish between $0, \pi$ phase shifts, so bits 


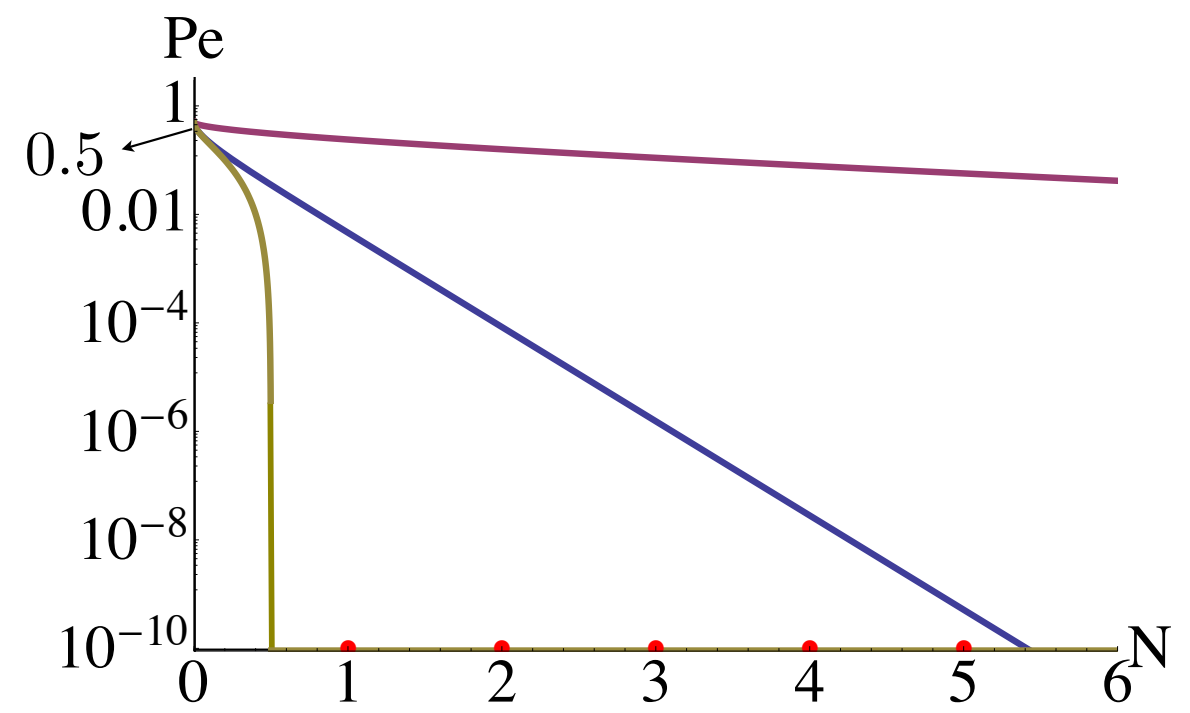

- Coherent State input with Homodyne Receiver

- Coherent State input with Dolinar Receiver $\}|\alpha|^{2}=N_{\text {avg }}$

- Optimum state

- $|2 N\rangle_{a}|0\rangle_{b}$ with Joined detection Receiver

Figure 3.8: Pe vs $N=N_{\text {avg }}$, for BPSK encoding using $(0, \pi)$ phase shifts for various experimental schemes, the coherent state probe with Homodyne and Dolinar receivers, the absolute optimum state for BPSK proposed by Nair, et.al [67], and the Fock state with Joint detection receiver. 
of information $0(1)$ is encoded as $0(\pi)$ phase shifts. But, our proposed interferometer can distinguish between various set of phases, say $\left(0, \frac{\pi}{2}\right)$, and each of these sets can be used for encoding 0(1) bits of information. For the sake of comparison, we now focus on our interferometer performance at $(0, \pi)$ phase shifts. we have already shown that MZI fed with $|2 n\rangle_{a}|0\rangle_{b}$ has optimum performance on these set of phase shifts, so in Fig. 3.8, we compare the behavior of coherent state interferometers with MZI interferometer fed with $|2 n\rangle_{a}|0\rangle_{b}$.

\subsubsection{3-ary phase discrimination, $\mathrm{M}=3$}

Now, we consider the problem of discriminating between three optical phase shifts employing our proposed interferometer. We apply one of the three phases $\left(0, p_{1}, p_{2}\right)$ with equal probability, then try to guess the phase shift based on the $J_{z}$ measurement outcome. Again, the probability of error $(\mathrm{Pe})$, is a natural criteria to measure the performance of the phase discrimination. For $M=3$ the Probability of error in Eq. (3.30) can be expressed as:

$$
P e=\frac{1}{3}\left(p_{\hat{\theta} \mid \theta}\left(0 \mid p_{1}\right)+p_{\hat{\theta} \mid \theta}\left(0 \mid p_{2}\right)+p_{\hat{\theta} \mid \theta}\left(p_{1} \mid 0\right)+p_{\hat{\theta} \mid \theta}\left(p_{1} \mid p_{2}\right)+p_{\hat{\theta} \mid \theta}\left(p_{2} \mid 0\right)+p_{\hat{\theta} \mid \theta}\left(p_{2} \mid p_{1}\right)\right)
$$

We loop through all phases $p_{1}$, and $p_{2}$, in the whole range of $0<p_{1}, p_{2}<2 \pi$ and calculate the probability of error for all possible combinations of $\left(0, p_{1}, p_{2}\right)$, Fig. 3.9 . The probability of error very much depends on the choice of phases. We go through 


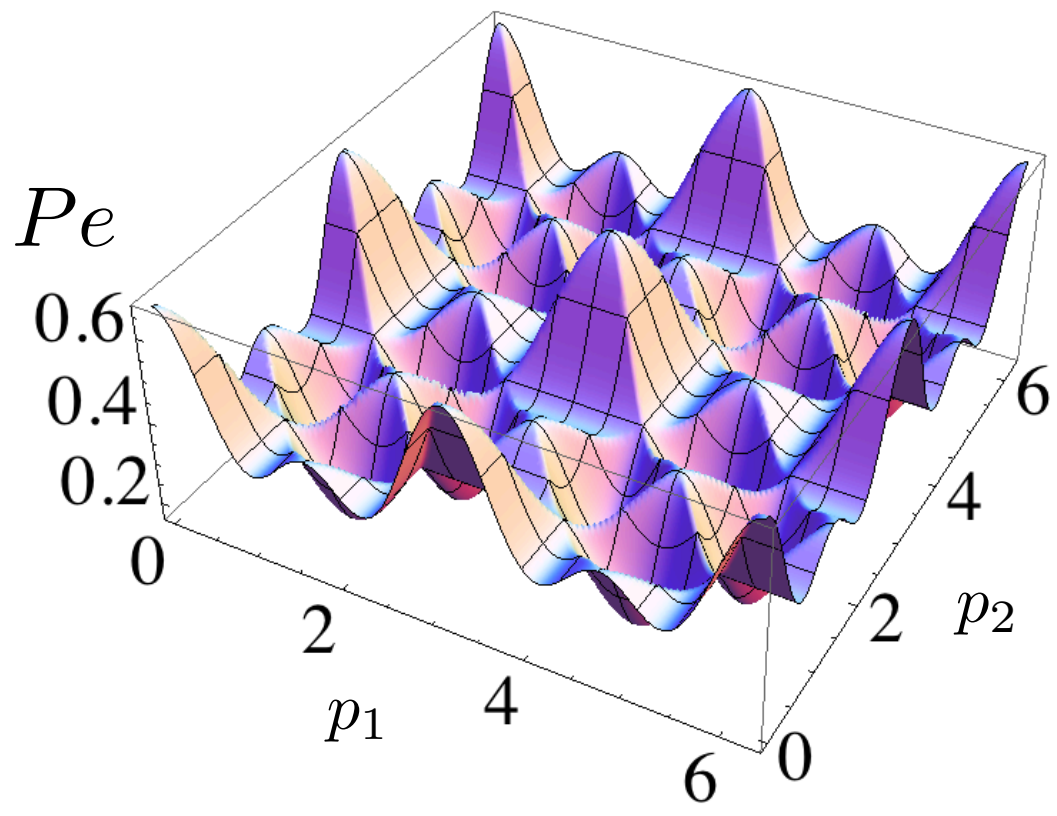

Figure 3.9: Probability of error (Pe) vs phase shifts $p_{1}$ and $p_{2}$, for optical phase discrimination between three phase shifts $\left(0, p_{1}, p_{2}\right)$. Interferometer is fed with photons prepared in $|2\rangle_{a}|2\rangle_{b} \Leftrightarrow|2,0\rangle_{z}$.

all Pe values and pick the optimum phases which gives the minimum probability of error. For example, for the interferometer fed with photons in $|2\rangle_{a}|2\rangle_{b}$ which is equivalent to the effective spin $|2,0\rangle_{z}$, the minimum probability of error is $P e=0.16$ and the optimum phases corresponding this $P e_{\min }$, are $\left(0, p_{1}, p_{2}\right)=\left(0, \frac{\pi}{4}, \frac{\pi}{2}\right)$.

As we have seen earlier in 2-ary phase discrimination problem, $M=2$, the interferometer performance very much depends on the form of the input state. In the following we compare the interferometer fed with photons in all types of the effective spins and then calculate their optimum phase values.

Fig. 3.10 compares the interferometer with four photons prepared in $|3\rangle_{a}|1\rangle_{b}$ and $|4\rangle_{a}|0\rangle_{b}$ states, these Fock states are equivalent to the effective spins $|2,1\rangle_{z}$ and 

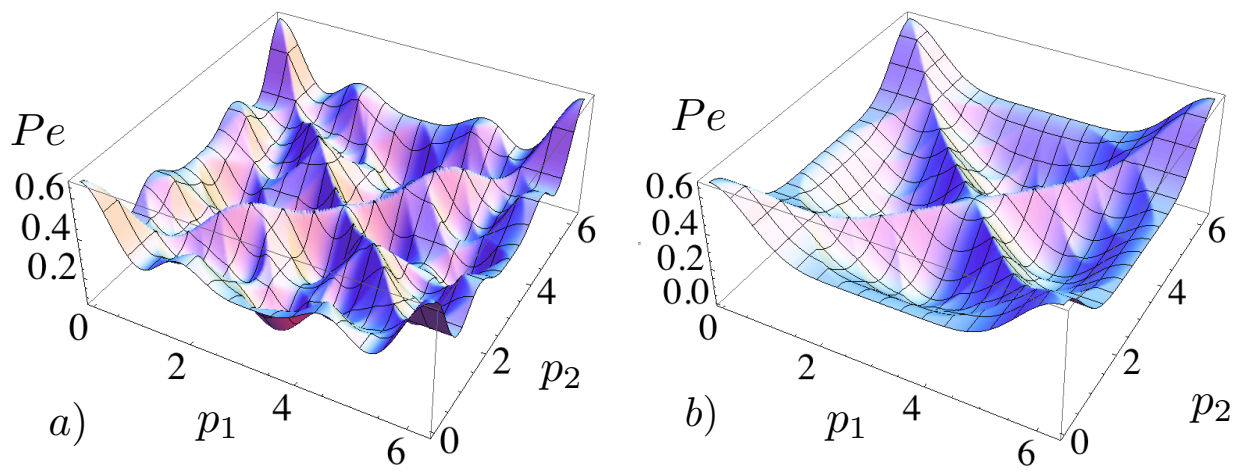

Figure 3.10: Probability of error (Pe) vs phase shifts $p_{1}$ and $p_{2}$, for optical phase discrimination between three phase shifts $\left(0, p_{1}, p_{2}\right)$. Interferometer is fed with photons prepared in a) $|3\rangle_{a}|1\rangle_{b} \Leftrightarrow|2,1\rangle_{z}$. b) $|4\rangle_{a}|0\rangle_{b} \Leftrightarrow|2,2\rangle_{z}$

$|2,2\rangle_{z}$ in the Schwinger representation. Similar to the $M=2$ case, interferometers fed with effective spins with lower $\mu$ values have more oscillation in their Pe diagram, with local minimas occurring at smaller phases $p_{1}$ and $p_{2}$, so they are more appropriate for discriminating between smaller phases. In other hand, the Pe performance for effective spins with higher $\mu$ values are more flat and have minimums at higher phases. As an example, in the case of $j=2$ (Fig. 3.9), the minimum error probability for effective spin $|2,2\rangle_{z}$ occurs at $\left(0, p_{1}, p_{2}\right)=\left(0, \frac{\pi}{2}, \pi\right)$ which should be compare to $\left(0, p_{1}, p_{2}\right)=\left(0, \frac{\pi}{4}, \frac{\pi}{2}\right)$ for effective spin $|2,0\rangle_{z}$.

We further study the behavior of interferometer with different input states and calculate their optimum phase values for higher photon average numbers as input to the interferometer and consequently on the signal probe. We list all optimum phases for different input states in the chart in Fig. 3.11

Unlike, the $M=2$ case, in the 3-ary phase discrimination, the Pe does not 


\begin{tabular}{|c|c|c|c|c|}
\hline Input State & $N_{a v g}$ & $\left(0, p_{1}, p_{2}\right)$ & $P e$ & $M I$ \\
\hline$|2\rangle_{a}|2\rangle_{b} \Leftrightarrow|2,0\rangle_{z}$ & 2 & $(0, \pi / 4, \pi / 2)$ & 0.160 & 0.93 \\
\hline 3$\left.\left.\rangle_{a} 1\right\rangle_{b} \Leftrightarrow 2,1\right\rangle_{z}$ & 2 & $(0, \pi / 2, \pi)$ & 0.160 & 0.97 \\
\hline 4$\left.\left.\rangle_{a} 0\right\rangle_{b} \Leftrightarrow 2,2\right\rangle_{z}$ & 2 & $(0, \pi / 2, \pi)$ & 0.040 & 1.35 \\
\hline 3$\left.\left.\rangle_{a} 3\right\rangle_{b} \Leftrightarrow 3,0\right\rangle_{z}$ & 3 & $(0,0.67, \pi / 2)$ & 0.140 & 1.13 \\
\hline 4$\left.\left.\rangle_{a} 2\right\rangle_{b} \Leftrightarrow 3,1\right\rangle_{z}$ & 3 & $(0, \pi / 2, \pi)$ & 0.008 & 1.50 \\
\hline 5$\left.\left.\rangle_{a} 1\right\rangle_{b} \Leftrightarrow 3,2\right\rangle_{z}$ & 3 & $(0,2.32,3.16)$ & 0.005 & 1.55 \\
\hline 6$\left.\left.\rangle_{a} 0\right\rangle_{b} \Leftrightarrow 3,3\right\rangle_{z}$ & 3 & $(0, \pi / 2, \pi)$ & 0.048 & 1.30 \\
\hline 4$\left.\left.\rangle_{a} 4\right\rangle_{b} \Leftrightarrow 4,0\right\rangle_{z}$ & 4 & $(0,0.55,1.2)$ & 0.120 & 1.12 \\
\hline 5$\left.\left.\rangle_{a} 3\right\rangle_{b} \Leftrightarrow 4,1\right\rangle_{z}$ & 4 & $(0,1.2, \pi)$ & 0.005 & 1.52 \\
\hline 6$\left.\left.\rangle_{a} 2\right\rangle_{b} \Leftrightarrow 4,2\right\rangle_{z}$ & 4 & $(0,0.6, \pi)$ & 0.002 & 1.55 \\
\hline 7$\left.\left.\rangle_{a} 1\right\rangle_{b} \Leftrightarrow 4,3\right\rangle_{z}$ & 4 & $(0,0.66, \pi)$ & 0.003 & 1.56 \\
\hline 8$\left.\left.\rangle_{a} 0\right\rangle_{b} \Leftrightarrow 4,4\right\rangle_{z}$ & 4 & $(0, \pi / 2, \pi)$ & 0.003 & 1.41 \\
\hline 5$\left.\left.\rangle_{a} 5\right\rangle_{b} \Leftrightarrow 5,0\right\rangle_{z}$ & 5 & $(0,0.42, \pi / 2)$ & 0.096 & 1.20 \\
\hline 6$\left.\left.\rangle_{a} 4\right\rangle_{b} \Leftrightarrow 5,1\right\rangle_{z}$ & 5 & $(0,1, \pi)$ & 0.005 & 1.54 \\
\hline 7$\left.\left.\rangle_{a} 3\right\rangle_{b} \Leftrightarrow 5,2\right\rangle_{z}$ & 5 & $(0,0.48, \pi)$ & 0.003 & 1.56 \\
\hline$|8\rangle_{a}|2\rangle_{b} \Leftrightarrow|5,3\rangle_{z}$ & 5 & $(0,0.45, \pi)$ & 0.024 & 1.40 \\
\hline$\left.\left.|9\rangle_{a} 1\right\rangle_{b} \Leftrightarrow 5,4\right\rangle_{z}$ & 5 & $(0,0.64, \pi)$ & 0.00004 & 1.58 \\
\hline$|10\rangle_{a}|0\rangle_{b} \Leftrightarrow|5,5\rangle_{z}$ & 5 & $(0, \pi / 2, \pi)$ & 0.00068 & 1.57 \\
\hline 6$\left.\left.\rangle_{a} 6\right\rangle_{b} \Leftrightarrow 6,0\right\rangle_{z}$ & 6 & $(0,1.32,2.8)$ & 0.088 & 1.20 \\
\hline 7 $\left.\left.\rangle_{a} 5\right\rangle_{b} \Leftrightarrow 6,1\right\rangle_{z}$ & 6 & $(0,1.32,3.16)$ & 0.004 & 1.54 \\
\hline 8$\left.\left.\rangle_{a} 4\right\rangle_{b} \Leftrightarrow 6,2\right\rangle_{z}$ & 6 & $(0,0.38, \pi)$ & 0.002 & 1.56 \\
\hline 9$\left.\left.\rangle_{a} 3\right\rangle_{b} \Leftrightarrow 6,3\right\rangle_{z}$ & 6 & $(0,0.42, \pi)$ & 0.00007 & 1.58 \\
\hline$\left.|10\rangle_{a}|2\rangle_{b} \Leftrightarrow 6,4\right\rangle_{z}$ & 6 & $(0,2.68, \pi)$ & 0.00023 & 1.58 \\
\hline 11$\left.\left.\rangle_{a} 1\right\rangle_{b} \Leftrightarrow 6,5\right\rangle_{z}$ & 6 & $(0,2.56, \pi)$ & 0.000066 & 1.58 \\
\hline$\left.|12\rangle_{a}|0\rangle_{b} \Leftrightarrow 6,6\right\rangle_{z}$ & 6 & $(0,1.56, \pi)$ & 0.0002 & 158 \\
\hline
\end{tabular}

Figure 3.11: Minimum Probability of error (Pe) and the Mutual Information (MI) for optimal phases $\left(0, p_{1}, p_{2}\right)$, for various input states. 
reach the exact zero, which means our interferometer is not a perfect machine for distinguishing between three quantum states. However, by increasing the average photon numbers $N_{\text {avg }}$ and carefully choosing the appropriate effective spin $|j, \mu\rangle$, one can achieve Pe with arbitrary small value. Once again, the above-mentioned chart demonstrates that, smaller $\mu$ values are always good candidates for discriminating between phases $\left(p_{1}, p_{2}<\pi\right)$. But, if discriminating between smaller phases is not of any interest then other types on input states with effective spin $|j, \mu\rangle(\mu>0)$ are better candidates and can perform phase discrimination with less error.

Looking at the chart, one can conclude that, among all effective spins with constant

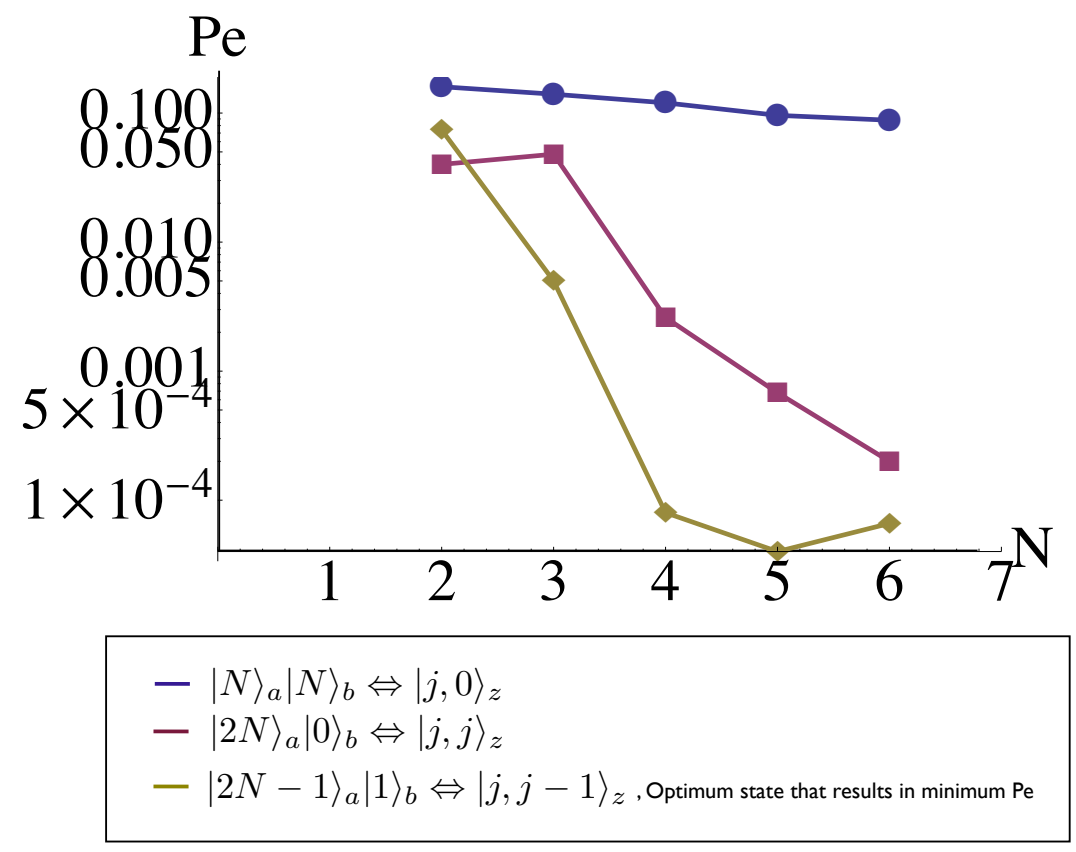

Figure 3.12: Minimum Probability of error (Pe) vs $\mathrm{N}$ for $\mathrm{M}=3$ and for all forms of input states. The effective spin $|j, j-1\rangle_{z}$ shows the best performance.

$j$, the spin $|j, j-1\rangle_{z}$ have the best performance. Fig. 3.12, demonstrate the Pe vs 
$N=N_{\text {avg }}$ for effective spins $|j, 0\rangle_{z},|j, j\rangle_{z}$ and $|j, j-1\rangle_{z}$.

We also consider the performance of the interferometer for discriminating between $M>3$ optical phases. In fact, the interferometer performance degrades with higher values of $\mathrm{M}$ and does not seem to be a good candidate for discriminating between more that three phases. To show that, we introduce a quicker method for calculating the Pe directly from the probability distributions $d_{m, m^{\prime}}^{j}(p)$, this method is more straightforward compare to the Mathematica programs explained earlier, but only works for effective spin $|j, 0\rangle$ and for discriminating between $M=j+1$ optical phases.

For the i-th row of any general selection rule chart, similar to the one presented in the Fig. 3.13 , elements can be written as $\left(\beta_{i 0}, \ldots . \beta_{i k}, \ldots ., \alpha_{i, k_{\alpha}}, \ldots \ldots ., \beta_{i j}\right)$, with $\alpha_{i, k_{\alpha}}>$ $\beta_{i k} \forall k$. we then define:

$$
\begin{aligned}
\beta_{i} & =\sum_{k} \beta_{i k} \\
D_{i} & =\alpha_{i}-\beta_{i}=d_{m, m_{k_{\alpha}}^{\prime}}^{j}\left(p_{i}\right)-\sum_{k \neq k_{\alpha}} d_{m, m_{k}^{\prime}}^{j}\left(p_{i}\right)
\end{aligned}
$$

The value of $D_{i}, \alpha_{i}$ and $\beta_{i}$ depends on the choice of phases $p_{i}$ on each row, so we write them as a function of $p_{i}$ as $D\left(p_{i}\right), \alpha\left(p_{i}\right)$ and $\beta\left(p_{i}\right)$. Since the probability of measuring all possible measurement outcomes is normalized to one, then $\alpha\left(p_{i}\right)+$ $\beta\left(p_{i}\right)=1 \forall i$. Phases with $\alpha\left(p_{i}\right)=1$ and $\beta\left(p_{i}\right)=0 \forall(i, k)$, results in the perfect phase discrimination. In most cases $\beta\left(p_{i}\right) \neq 0$ and achieving error free phase discrimination is impossible so we are interested to find phases $p_{i}$ that maximize $\alpha\left(p_{i}\right)$ and minimize the $\beta\left(p_{i}\right)$. In order to find optimum phases, we plot the function $D\left(p_{i}\right)=\alpha\left(p_{i}\right)-\beta\left(p_{i}\right)$ 


\begin{tabular}{|c|c|c|c|c|}
\hline$\theta$ & 0 & । & 2 & 3 \\
\hline$p_{1}$ & $\alpha_{1}$ & $\beta_{11}$ & $\beta_{12}$ & $\beta_{13}$ \\
\hline$p_{2}$ & $\beta_{20}$ & $\beta_{21}$ & $\alpha_{2}$ & $\beta_{23}$ \\
\hline$p_{3}$ & $\beta_{30}$ & $\alpha_{3}$ & $\beta_{32}$ & $\beta_{34}$ \\
\hline$p_{4}$ & $\beta_{40}$ & $\beta_{41}$ & $\beta_{42}$ & $\alpha_{4}$ \\
\hline \multicolumn{5}{|c}{$\downarrow$} \\
& $p_{1}$ & $\begin{array}{c}\downarrow \\
p_{3}\end{array}$ & $\begin{array}{c}\downarrow \\
p_{2}\end{array}$ & $p_{4}$ \\
\hline
\end{tabular}

Figure 3.13: The selection rule chart for discriminating between $M=4$ optical phase shifts with interferometer fed with $|3,0\rangle_{z}$ input spin. we have not listed negative values of $\mu^{\prime}$ in the chart, in fact, $d_{0, \mu^{\prime}}^{j}\left(p_{1}\right)=d_{0,-\mu^{\prime}}^{j}\left(p_{1}\right)$, so we do not need to consider $-\mu^{\prime}$ in separate columns and $\mu^{\prime}$ and $-\mu^{\prime}$ columns can be combined in one with $\beta_{i k}=$ $2 d_{0, \mu_{k}^{\prime}}^{j}\left(p_{1}\right)$ for $k \neq 0$.

for all phases and then pick phases that corresponds to the maximum $D\left(p_{i}\right)$. Note that, $k_{\alpha}$, the position of $\alpha_{i, k_{\alpha}}$ depends on the choice of the phase shift and is not required to be known in advance. We look at $D\left(p_{i}\right)$ for $0 \leq k_{\alpha} \leq j$ and then pick the optimum phases which corresponds to a local maximum. Each maximum corresponds to a minimum probability of error for $P e_{i}$. Therefore knowing the value of these maximums $D\left(p_{i}\right)$ for optimum phases $p_{i}$, one can calculate the probability of error as:

$$
P e=\frac{1}{M} \sum_{i} P e_{i}=\frac{1}{M} \sum_{i} \beta\left(p_{i}\right)=\frac{1}{M} \sum_{i} \frac{1-D\left(p_{i}\right)}{2}
$$

If maximum values of $D\left(p_{i}\right)$ drops below zero then the probability of error $P e \geq$ $1 / 2$, it is considered as big error in the phase estimation problem and means that 


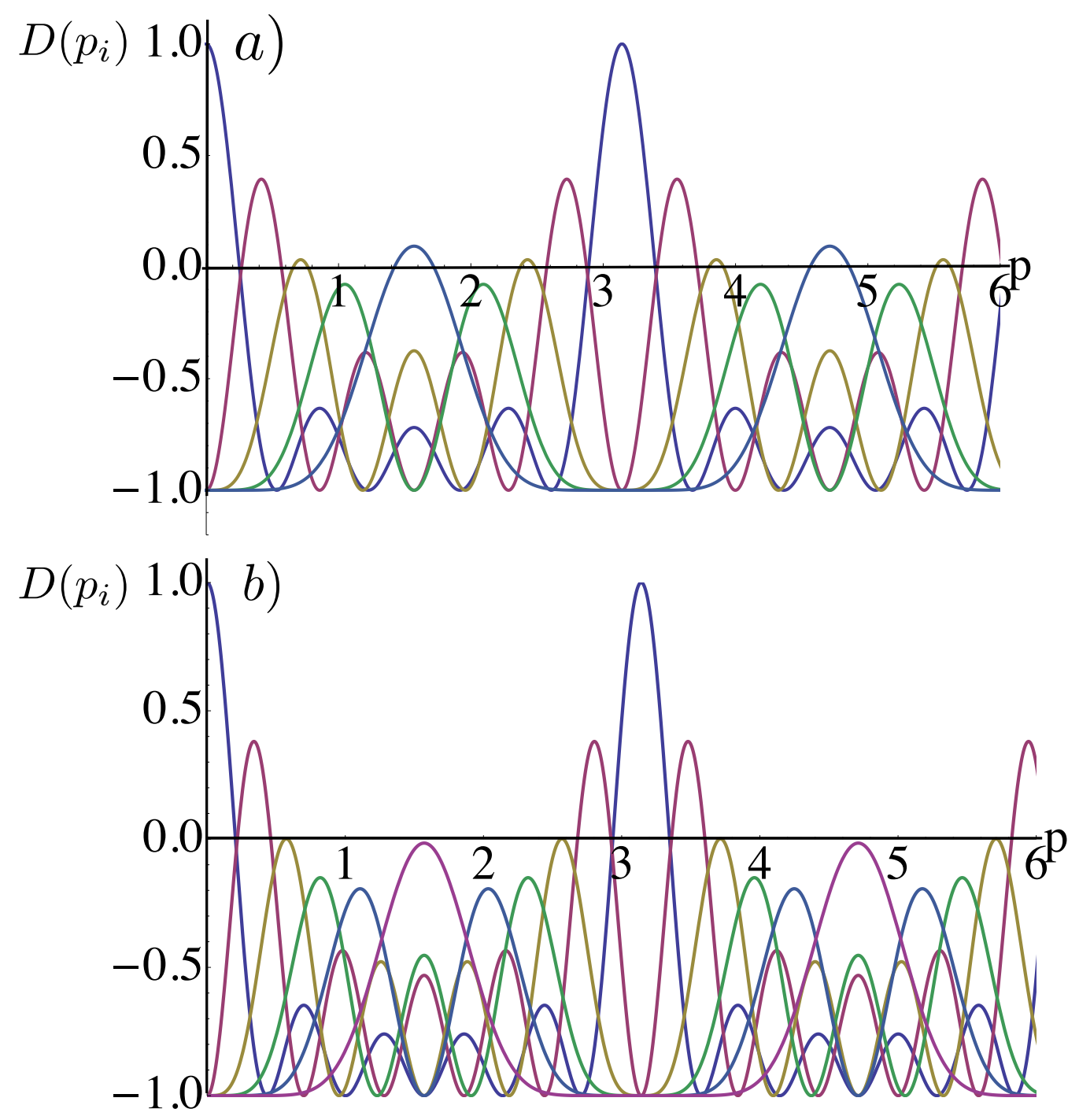

Figure 3.14: $\quad D\left(p_{i}\right)$ for all phases, the phases at which each maximum occurs is an optimum phase and the value of the maximum is inversely proportional to the probability of error, a) $M=4$ b) $M=6$ 
for these values the interferometer is not appropriate for this task. We have plotted all $D\left(p_{i}\right)$ s for $M=4,6$ in Fig. 3.14 , we can clearly see that the maximum values are dropping very quickly by increasing the $M$, so our proposed interferometer is not appropriate for discriminating between $M>3$ optical phases.

\subsection{Optical Reading}

One application of our study of error free optical phase discrimination is to the recently developed concept of quantum reading of a classical digital memory, which uses quantum optical probe state to read optically encoded digital memories such as in CDs and DVDs [68], 69]. Our proposed interferometry scheme can be employed to generate the quantum state probe to read optically encoded digital memories and the specified joint detection receive (JDR) can successfully record the encoded message.

Optical memories are highly reflective amplitude modulated memory surfaces in which digital information is encoded in pixels as tiny indentations known as "pits" and the areas between pits which are known as "lands". Digital information stored in the amplitude modulated memory surfaces can be read by shining a beam of light, in which the information encodes on the optical phase shift of the reflected light probe as a bi-phase modulation, also known as the binary phase shifted keyed (BPSK) modulation and can later be recorded with photo diodes as the intensity difference upon interference with the reference light.

The classical capacity of optical reading is the amount of bits of information that can be reliably encoded and read per pixel, it is equivalent to the maximum attainable 


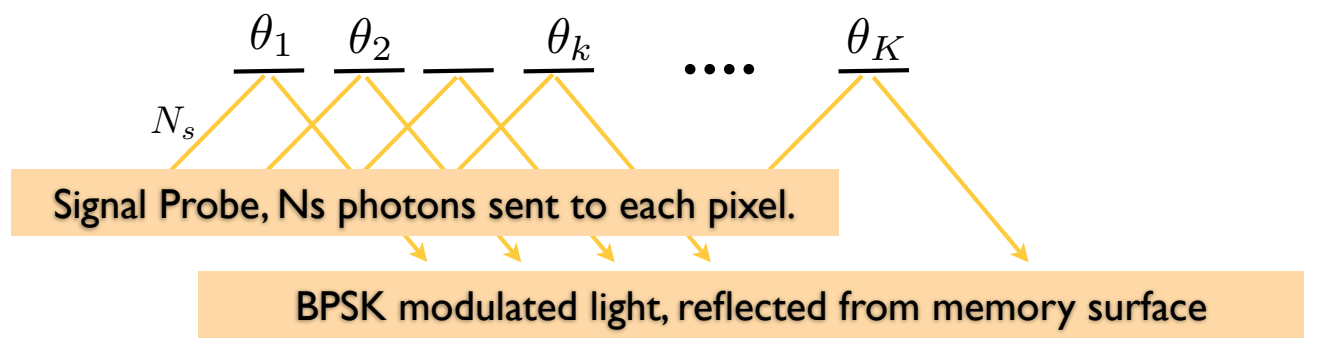

Figure 3.15: The setup for optical reading. $N_{s}$ photons are sent to each memory pixel of the amplitude modulated memory surface. The binary information is encoded on the reflected light as BPSK modulation.

mutual information (MI) between the applied phase shifts $\theta$ and the measured phase shifts $\hat{\theta}$ for each pixel:

$$
C\left(N_{s}\right)=\max I(\theta ; \hat{\theta})
$$

and the Photon Information Efficiency (PIE) is the number of bits read per signal photon:

$$
P I E=\frac{C\left(N_{s}\right)}{N_{s}}
$$

We calculate the capacity of optical reading assuming only one memory pixel, we adjust the Fock state interferometry scheme which is well studied for the problem of phase discrimination for the optical reading and then compare its performance with optical reading with coherent state and Homodyne or Dolinar receivers. We first calculate the mutual information $(\mathrm{MI}), I(\theta ; \hat{\theta})$ for all combination of $p_{1}, p_{2}$ phases and then look for optimum phase values which maximizes the mutual information, then we record these maximum values as the capacity of optical reading. 
Note that the average number of photons $N_{s}$ in the signal port is:

$$
\begin{gathered}
N_{s}=\left\langle n_{a}, n_{b}\left|U_{B S}^{\dagger} N_{a} U_{B S}^{\dagger}\right| n_{a}\right\rangle\left|n_{b}\right\rangle=\frac{1}{2}\left\langle n_{a}\left|\left\langle n_{b}\left|\left(a^{\dagger}+b^{\dagger}\right)(a+b)\right| n_{a}\right\rangle\right| n_{b}\right\rangle \\
=\frac{1}{2}\left\langle n_{a}\left|\left\langle n_{b}\left|\left(a^{\dagger} a+b^{\dagger} b\right)\right| n_{a}\right\rangle\right| n_{b}\right\rangle=\frac{1}{2}\left(n_{a}+n_{b}\right)=n=j
\end{gathered}
$$

We plotted the Mutual Information (MI) for TFSI fed with $n=1,2$ photons in Fig. 3.16. The maximum value of mutual Information (MI) reaches one for all types of effective input states and for all average photon numbers $N_{a v g}=n$. But optimum phases corresponding to the maximum MI is different for various types of effective spins.

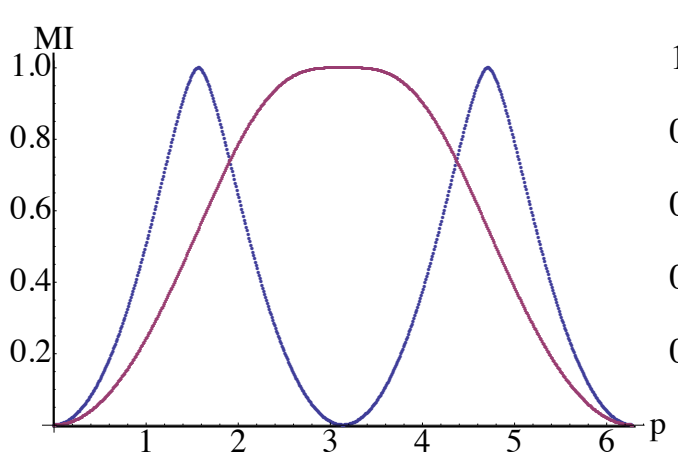

a)

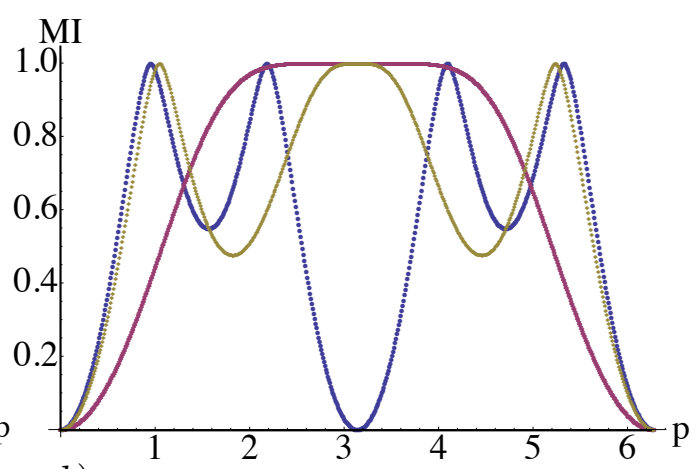

b)

$$
\begin{aligned}
& -|n\rangle_{a}|n\rangle_{b} \Leftrightarrow|j, 0\rangle_{z} \\
& -|2 n\rangle_{a}|0\rangle_{b} \Leftrightarrow|j, j\rangle_{z} \\
& -|n+m\rangle_{a}|n-m\rangle_{b} \Leftrightarrow|j, m\rangle_{z}, m=1
\end{aligned}
$$

Figure 3.16: The mutual information for optical reading. The binary information is encoded in optical phase shifts $(0, p)$. a) $n=1$ b) $n=2$

The classical capacity of a quantum channel is limited by the Holevo bound, which imposes an upper limit on the continued reliable rate of reading classical information 
from quantum channel [70], [71] . For the lossless phase only encoding the Holevo capacity can be written as [72]:

$$
C\left(N_{s}\right)=g\left(N_{s}\right)
$$

where:

$$
g(x)=(1+x) \log _{2}(1+x)-x \log _{2}(x)
$$

The Holevo capacity can be achieved using optimum probe state with optimum receiver design, However, there is no feasible experimental scheme to approach this limit. Common optical reading schemes implement coherent states of light along with Homodyne or heterodyne receivers. We also consider optical reading with coherent state and Dolinar receiver. For these binary channels the channel capacities can be written as:

$$
\begin{gathered}
C\left(N_{s}\right)=\max I(\theta ; \hat{\theta})=1-H(P e) \\
H(x)=-q \log _{2}(q)-(1-q) \log _{2}(1-q)
\end{gathered}
$$

Pe for Homodyne and Dolinar receivers are calculated in Eq. (3.49), Eq. (3.51). For the energy constraint issues, people are interested in experimental schemes for optical reading in which the bits of information read per photon is higher. So we compare the performance of various well known experimental schemes for optical reading based on their photon information efficiency (PIE) vs the capacity of optical reading (bits encoded per pixel) and the PIE vs $N_{s}$. 


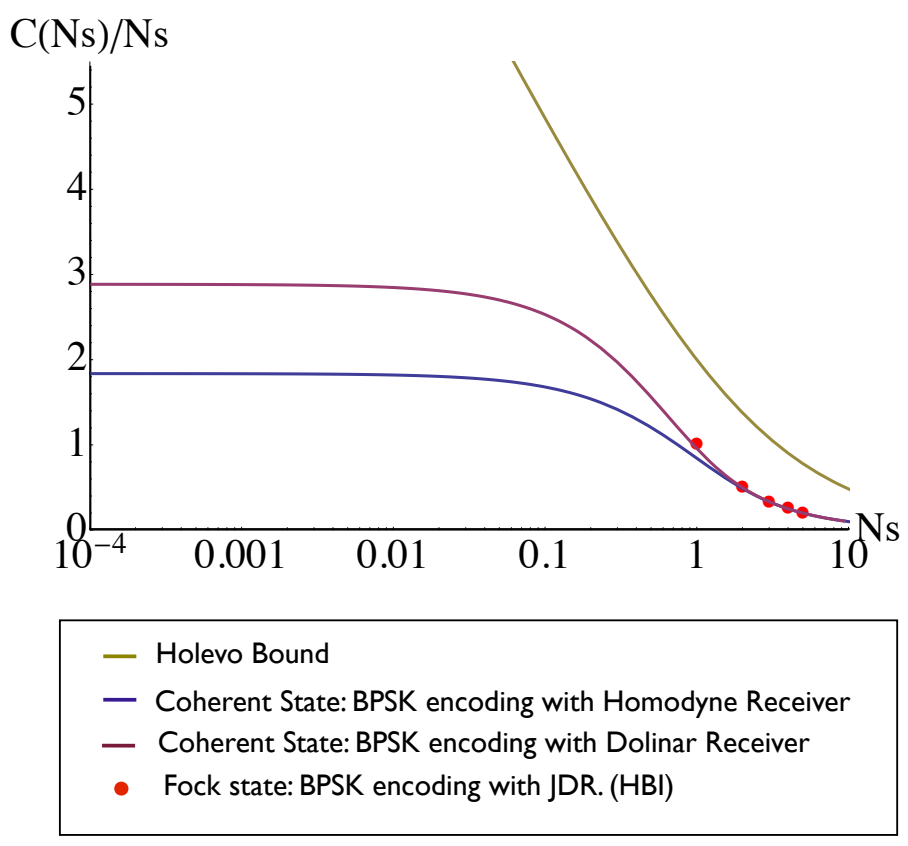

Figure 3.17: Photon information efficiency $C\left(N_{s}\right) / N_{s}$ versus $N_{s}$, for various interferometry schemes.

Fig. 3.17 and Fig. 3.18 compare the performance of optical reading implementing TFSI with various input photon numbers with the Holevo capacity and the capacity of binary phase encoding using coherent state probe with Homodyne and Dolinar receivers.

The performance of our interferometer is slightly better than the Dolinar receiver for $n=1$. We also include the gray area which corresponds to the best performance of conventional optical reading disks in the diagram which compares the PIE $=$ $c\left(N_{S}\right) / N_{s}$ vs the capacity of optical reading $C\left(N_{s}\right)$. Conventional optical reading techniques presented here use coherent state probe with on-off amplitude modulation and direct detection. Our scheme clearly outperform the conventional techniques and 
in most cases the coherent state inputs with Homodyne and Dolinar receivers.

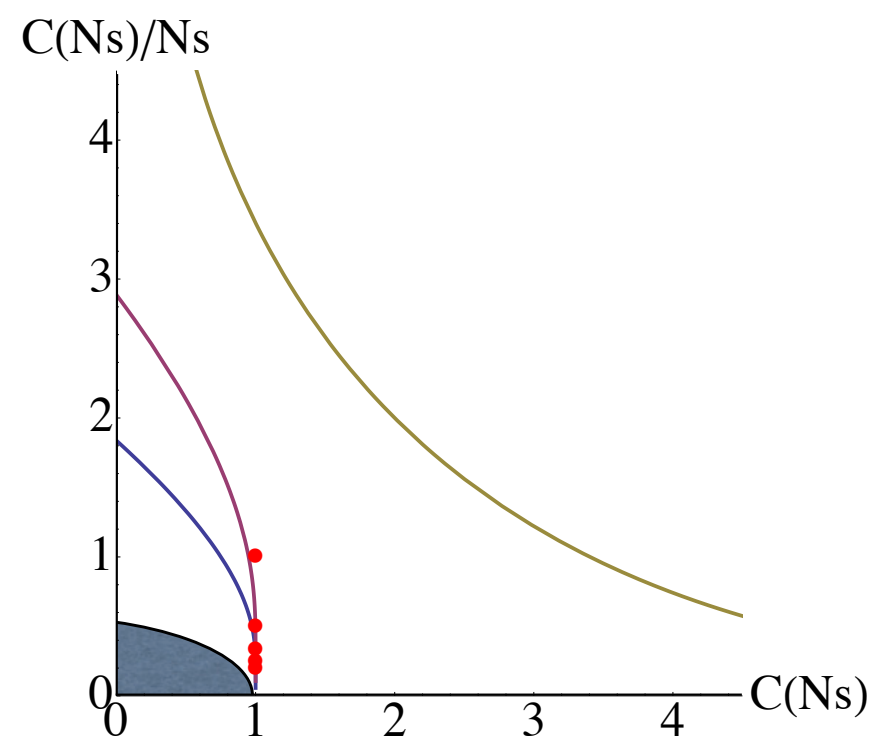

- Holevo Bound

- Coherent State: BPSK encoding with Homodyne Receiver

- Coherent State: BPSK encoding with Dolinar Receiver

- Fock state: BPSK encoding with JDR. (HBI)

$\square$ Coherent state: On off encoding with direct detection

Figure 3.18: Photon information efficiency (bits per photon) vs the encoding efficiency (bits encoded per pixel) for various input state and receiver strategies.

In classical optical reading, lands and pits need to at least have $\frac{\lambda}{4}$ height difference to be distinguishable with conventional light interferometry, $\lambda$ being the optical probe wavelength. In fact, $\frac{\lambda}{4}$ height difference results in a $\pi$ phase shift on the optical beam. Notably, our interferometry model is capable of distinguishing between smaller phase shifts,Fig. 3.19, and thus shorter height differences. In fact, TFSI enable us to read pits etched $\frac{\lambda}{8}$ deeper respect to lands, so our interferometry scheme realizes memory surfaces with shallower pits. 


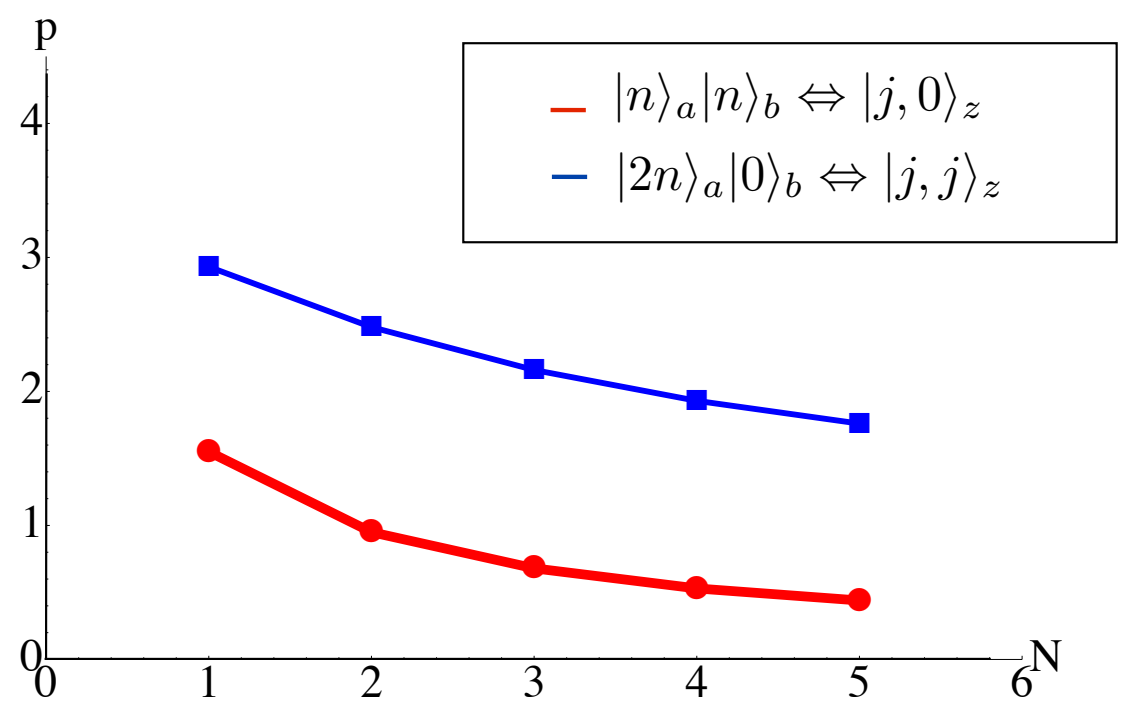

Figure 3.19: Optimum p for the MZI interferometer fed with two different effective spins, vs the average photon number $N=N_{s}$. Optimum phases are smaller for MZI fed with twin-Fock state and is higher for single ported MZI.

We also proposed an optical reading scheme with 3-ary phase shifted keyed (PSK) modulation, where information is encoded as three distinct phases shifts $\left(0, p_{1}, p_{2}\right)$, in each memory pixel. This can potentially results in a denser optical encoding.

Figure 3.20 illustrates the performance of MZI interferometer for 3-ary PSK phase encoding. The single ported MZI with $|j, j\rangle$ effective spin, can reach to higher optical capacities and can read $\log _{2}(3) \simeq 1.6$ bits of information per pixel with near zero probability of error using as few as two photons, Fig. 3.11. MZI with twin Fock states does not approach the maximum achievable capacity of optical reading but it allows us to encode information in smaller phases. 


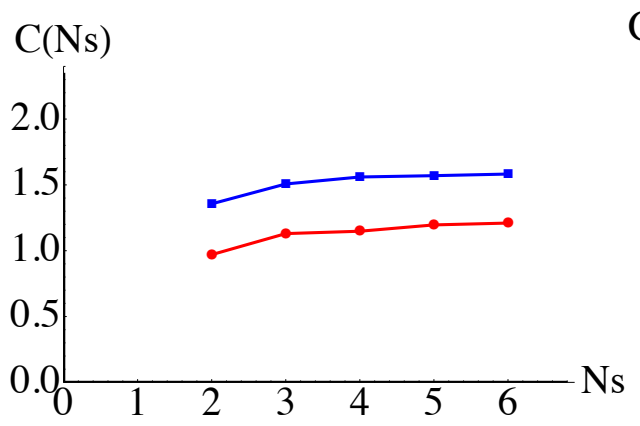

a)

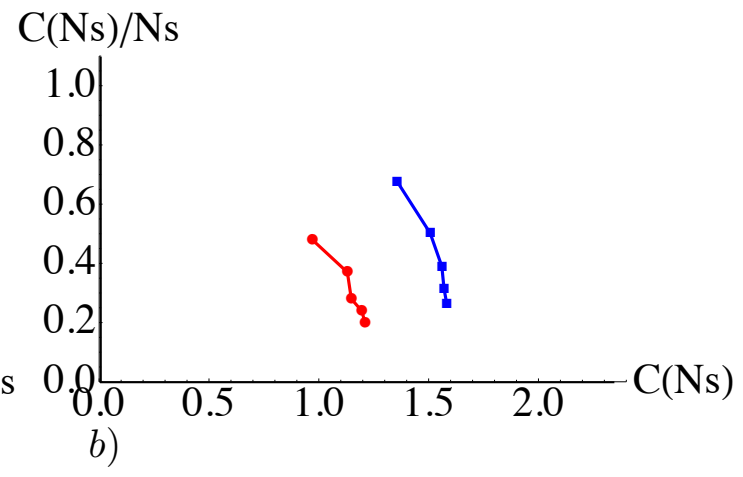

$-|n\rangle_{a}|n\rangle_{b} \Leftrightarrow|j, 0\rangle_{z}$

$-|2 n\rangle_{a}|0\rangle_{b} \Leftrightarrow|j, j\rangle_{z}$

Figure 3.20: The capacity of optical reading using Fock states with 3-ary PSK modulated phase encoding and joint detection receiver.

\subsection{Conclusion}

We have shown that employing the MZI interferometers fed with Fock states along with specific joint detection receiver provide an experimental scheme for M-ary phase discrimination on $M=2,3$. The probability of error for the $M=2$ case is always zero independent of the form of the input state, but for $M=3$ Pe very much depends on the form of the input state and is not exactly zero, We showed that using more and more photons help us to discriminate between smaller and smaller phase shifts and with less probability of error. We also showed that we can successfully discriminate between various phases by carefully designing the input Fock state and sending the specific effective spin. Our proposed interferometry scheme can also be implemented for reading digital classical memories using quantum light. The performance of our 
interferometer will outperform the performance of conventional optical reading techniques with coherent state inputs and direct detection, it also perform slightly better than coherent state input BPSK encoding with Homodyne and Dolinar receivers. 


\section{Chapter 4}

\section{Information-efficient and boundless reading of classical bits using single-photon source}

\subsection{Introduction}

Photons are ideal candidates for communicating information over long distances, they are also extensively implemented in optical reading of stored digital information, such as in CDs and DVDs. To achieve highly efficient communication and reading rates, researchers are interested to maximize the information content of the photon. There are many ways to increase the information capacity of a photon by encoding information on the various photon degrees of freedom, such as in the amplitude, phase, time, polarization and recently the orbital angular momentum of the photon [73].

In this chapter, we implemented an experimental scheme to exploit the phase of the photon to read boundless bits of digital information using a single photon. This scheme was first proposed by Saikat, et al in $[72$ and is an example of implementing single photons for obtaining the quantum advantages for various quantum information 
applications, here to increase the efficiency in reading out information stored in a classical digital memory. Another theoretical proposal for quantum optical reading implementing a Mach-Zehnder interferometer fed with Fock states was proposed and discussed earlier in section 3.6 .

In this experiment, we made use of the $M \times M$-port generalized Mach-Zehnder interferometer (MZI) and showed how the optical phase can be used to read $\log _{2}(M)$ bits of classical digital information using a single photon. It allowed us to attain high photon information efficiency (PIE), where the PIE is the number of bits read per signal photon.

This chapter is organized as follow, we explained about the principle of interferometer in the first section, then discussed about the quality of the single-photon source by calculating the degree of second-order coherence $g^{2}(0)$. Having highly efficient PNR detectors gave us the opportunity to directly measure the $g^{2}(0)$ as low as 0.03 .

Finally in section three, we looked at the interferometer performance and demonstrated the actual value of the PIE that we achieved considering all experimental imperfections.

\subsection{Principle of the experiment}

The $M \times M$-port generalized MZI is depicted in Fig. 4.1. We used the freedom on the optical phase shifts between arms of the interferometer to generate $M$ binary phase-shift keyed (BPSK) code word from the Hadamard code.

The single photon interfered in the $M \times M$-port beam splitter, experienced one of 


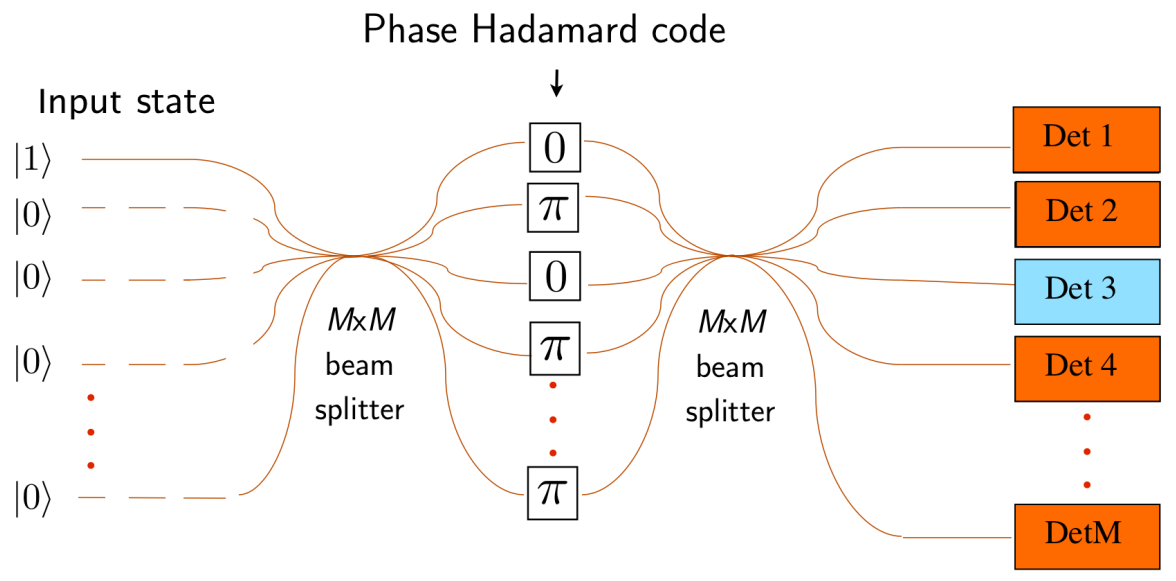

Figure 4.1: Principle of high-PIE optical reading scheme. An $M \times M$-port generalized MZI is fed with a single photon. A Hadamard phase shift array of $0 \pi 0 \pi \ldots . . \pi$ as M-long binary code word, is applied between interferometer arms and cause the photon to constructively interfere on the third output port of the interferometer and so appear on the third PNR detector.

the $M$ Hadamard phase coded memory, and then recombined with another $M \times M$ port beam splitter. The $M \times M$-port beam splitter is a symmetric generalized beam splitter with $M$ input and $M$ output ports and can be realized using linear network of standard $2 \times 2$ beam splitters. Zukowski, et al showed that such generalized beam splitter can be constructed using two ported beam splitters and can produce higher dimensional Einstein-Podolsky-Rosen (EPR) correlations [74.

The $M \times M$-port beam splitter (MBS) which is also called W state transmitter, evolve the single photon wave function and prepare it in the spatially entangled non classical $M$ mode W-state.

$$
|W\rangle=\frac{|10 \ldots 0\rangle+|01 \ldots 0\rangle+\ldots+|00 \ldots 1\rangle}{\sqrt{M}}
$$

The single photon prepared in the $\mathrm{W}$-state then experienced a Hadamard phase 
shifted code word between arms of the interferometer and depending on the Hadamard code applied, the single photon interfered constructively at one of the $M$ outputs of the interferometer and destructively on other $M-1$ output ports. Therefore by tracking the photon and knowing where it clicks one can distinguish between $M$ different Hadamard phase shifts and consequently read $\log _{2}(M)$ bits of classical information.

Here as a proof of principle, we experimentally realized the simpler case of the $4 \times 4$-port interferometer which is capable of encoding and transmitting 2 bits of information using a single photon $(\mathrm{PIE}=2)$. However, the scalability of the interferometer to the more general case of the $M \times M$-port interferometer is feasible using the current technology in $M \times M$-port integrated devices. Such devices allow the design of MBS on integrated silicon chips and with superior performance [75]. They will dramatically reduce the complexity of the set up required to generate arbitrary large quantum states, such as W-states.

\subsubsection{Interferometer}

The experimental realization of this $4 \times 4$-port W-state interferometer can be explained in 3 parts - the $\mathrm{W}$-state creation, the phase array and the green machine receiver. For accurate imaging of the phase array, the relative phases of all 4 modes must be stabilized when they are moving from one part of the interferometer. Rather than effect a 4-spatial-path stabilization system, we implemented a compact polarization encoded system which required only a single path stabilization. As in Fig Fig. 4.2, the input mode was sent in and split twice, first at PBS 1, then again at 


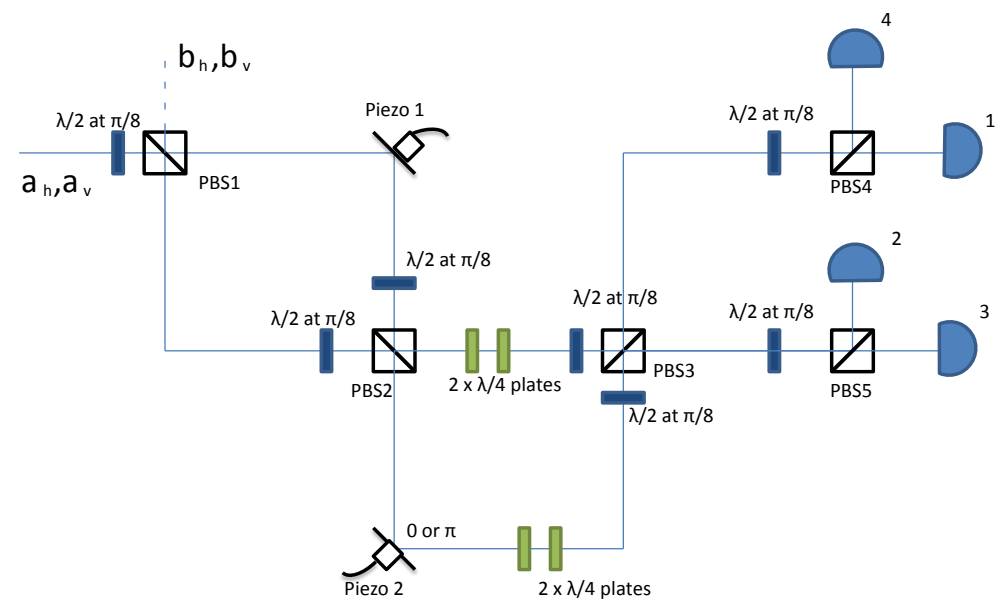

Figure 4.2: Polarization implementation of 4-port interferometer with only two optical paths.

PBS 2. Note that at PBS 2, 2 optical modes are mixed with a vacuum mode each to give 4 optical modes. There are two spatial modes in each PBS output and each has two orthogonal polarization modes. In the case of a single photon input, this creates a W-state. The polarization modes in the same spatial mode undergo the same path length and therefore the same phase shifts. Therefore only the two spatial modes need to be phase stabilized with respect to each other which is done using piezo 1 . The phase array is created using piezo 2 and 4 quarter wave plates. Piezo 2 shifts both modes of one optical path with respect to the other path, while the 2 quarter wave plates in each path are used to effect a relative phase shift of either 0 or $\pi$ between 
the 2 polarization modes in the same spatial path. Finally we interfere the modes at the other beam splitter array constructed of 4 beamsplitter, implemented compactly in our setup using 3 polarization beam splitters. Each mode interferes with every other mode and depending on the phase array, exit into the 4 detectors. If the phase array is a Hadamard code, all the input must exit entirely through one of the ports and the other three detectors will record zero. However this is the ideal situation and in practice, loss and other constraints distort the results.

\subsection{Single-photon source}

Any single-mode quantum state (pure or mixed), when used as the imaging probe in the proposed $M \times M$ interferometer, appears at one of the $M$ output ports depending upon the Hadamard codeword in the interferometer, so one can read same bits of information with higher photon numbers. The Photon Information Efficiency (PIE) then depends on the average number of photons $\bar{N}$ used to read the information.

Therefore in order to achieve to higher information efficiencies, more bits of information read per photon, a true single-photon source with $\bar{N}=1$ and $\Delta N=0$ is required.

An extensive effort was conducted in our lab to generate high fidelity single photon sources, as was discussed earlier in chapter 2, In order to examine the quality of our single-photon source we studied the photon number statistics of the heralded source and directly calculated the quantum degree of second-order coherence $g^{(2)}(0)$, with 
the method which was explained in detail in section 1.2.1.

\subsubsection{Quantum degree of second-order coherence $g^{(2)}(0)$ for heralded single-photon source}

The second-order autocorrelation function $g^{(2)}(0)$ is ideally zero for a true singlephoton source and is equivalent to negligible emission of photons in higher photon numbers and is highly desired in the current and many other quantum optic experiments. However, $g^{(2)}(0)$ is not exactly equal to zero for real experimental situations where losses in optical elements and detector imperfections play an important role, therefore $g^{(2)}(0) \neq 0$ and lower the $g^{(2)}(0)$ values corresponds to the better quality single-photon sources. For the sake of comparison, we also calculated the $g^{(2)}(0)$ for coherent and thermal states.

We analyzed the photon number statistics of the desired state on the TES, see Fig. 4.3, The data presented in this graph is over one trace, we analyzed many of these traces to have more accurate statistics.

Each trace is over $\Delta t=13 \mathrm{~ms}$ in $\delta t=3 \mu \mathrm{s}$ bursts (TES resolution or cool down time), we then analyzed up to 1000 such traces to have good statistics. The total number of bins then are: Eq. 1.43

$$
N_{\text {bins }}=\frac{\Delta t}{\delta t} \times 1000=\frac{13 m s}{3 \mu s} \times 1000=4.33 \times 10^{6}
$$

We first calculated the $g^{(2)}(0)$ for the attenuated seed beam as a coherent source of light. Counting average numbers of $n_{1}$ and $n_{2}$ and so $p_{1}$ and $p_{2}$ and plugging them 


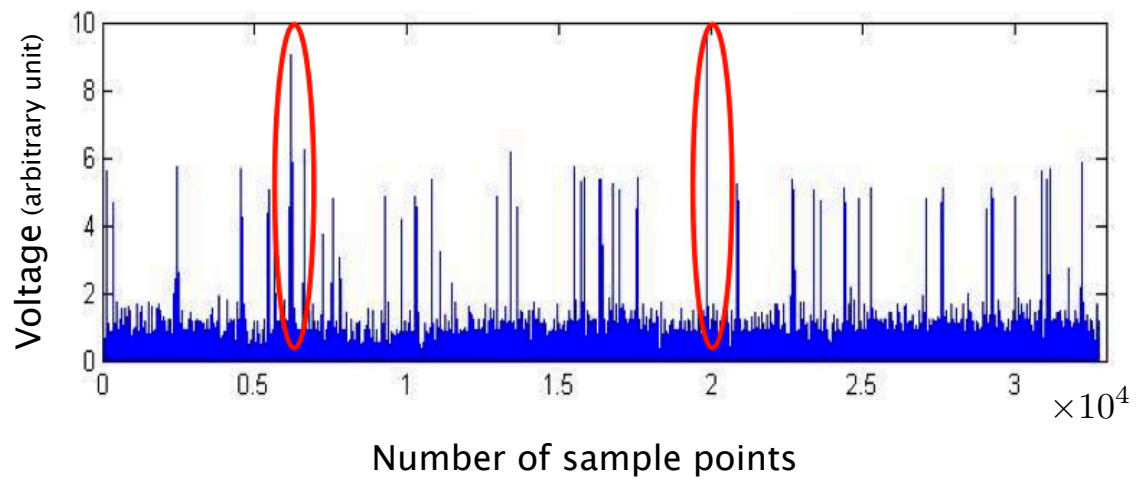

Figure 4.3: Raw TES data over 13ms (one trace). Single photon and two photon peak's height are respectively about $5 \mathrm{~V}$ and $10 \mathrm{~V}$. Red circles shows two photon peaks which will results in $g^{(2)}(0) \neq 0$, Eq. (1.48).

into Eq. 1.49), allowed us to calculate $g^{(2)}(0)=1 \pm 0.001$, as was expected for a coherent state source Eq. 1.50 . We did not have enough statistics, so $g^{(2)}(0)$ is not exactly one but is very close to unity.

We then considered the $g^{(2)}(0)$ of a thermal source. Tracing over one of the SPDC modes, say the signal, prepared the idler mode in a thermal state. The $g^{(2)}(0)$ of a thermal source should be equal to 2 . Here, we evaluated the photon number statistics of the idler mode on TES, and calculated $g^{(2)}(0)=2.2 \pm 0.2$.

Finally, we consider the heralded single-photon state. We calculated the $g^{(2)}(0)$ few times and every time on different data sets and with a particular pump power, number of traces and measurement window as listed in the table 4.1. The value of $g^{(2)}(0)$ depends on different parameters and ranges from $0.03-0.181$.

For a perfectly correlated sources, measuring a single photon on the heralding channel prepares a single photons on the heralded channel, with no zero or two pho- 


\begin{tabular}{|c|c|c|c|c|c|c|c|}
\hline \hline$I_{\text {pump }}$ & heralding & samples & \#traces & $n_{1}$ & $n_{2}$ & $N_{\text {bin }}$ & $g^{2}(0)$ \\
\hline $175 \mu \mathrm{W}$ & Ch 1 & 6 & 1064 & 6676 & 44 & 30456 & 0.059 \\
\hline $175 \mu \mathrm{W}$ & Ch 2 & 6 & 1064 & 6676 & 39 & 339136 & 0.058 \\
\hline $175 \mu \mathrm{W}$ & Ch 1 & 5 & 1064 & 6535 & 35 & 29359 & 0.047 \\
\hline $175 \mu \mathrm{W}$ & Ch 2 & 5 & 1064 & 6535 & 21 & 31115 & 0.030 \\
\hline $230 \mu \mathrm{W}$ & Ch 1 & 6 & 859 & 9647 & 115 & 42298 & 0.099 \\
\hline $230 \mu \mathrm{W}$ & Ch 2 & 6 & 859 & 9647 & 111 & 47519 & 0.108 \\
\hline $400 \mu \mathrm{W}$ & Ch 1 & 6 & 114 & 1287 & 16 & 10140 & 0.189 \\
\hline $400 \mu \mathrm{W}$ & Ch 2 & 6 & 114 & 1287 & 18 & 7856 & 0.160 \\
\hline
\end{tabular}

Table 4.1: The $g^{(2)}(0)$ value calculated for heralded single photon state. The value in each row is calculated for a particular pump power, number of trace and measurement window.

tons contribution and results in $g^{(2)}(0)=0$. However, for imperfect experimental situations with lossy detection, $g^{(2)}(0)>0$. Figure 4.4 clearly shows the effect of imperfections on heralding on zero or two photons, it shows that many times there is a photon in the heralding channel but it's partner is missing on the heralded channel (black circles), or during detection process on the heralding channel a $|2\rangle_{\text {signal }}|2\rangle_{\text {idler }}$ state can lose a photon and become a $|1\rangle_{\text {signal }}|2\rangle_{\text {idler }}$, so heralding on single photon results in two photon on the heralded one (red circles) Fig. 4.4, the latter situation rapidly increase $g^{(2)}(0)$ from zero.

Increasing the pump power increased the squeezing parameter $r$ and then the probability of emitting photons in $|2\rangle_{\text {signal }}|2\rangle_{\text {idler }}$ state, so expanded the $g^{2}(0)$ value for the heralded single-photon state as presented in table 4.1 .

The other important parameter for the $g^{(2)}(0)$ value was the measurement window. TES detectors are not fast and have a response time of about $3 \mu s$, so at higher pump photon rates there was greater probability of pileups, pileups happened when the 


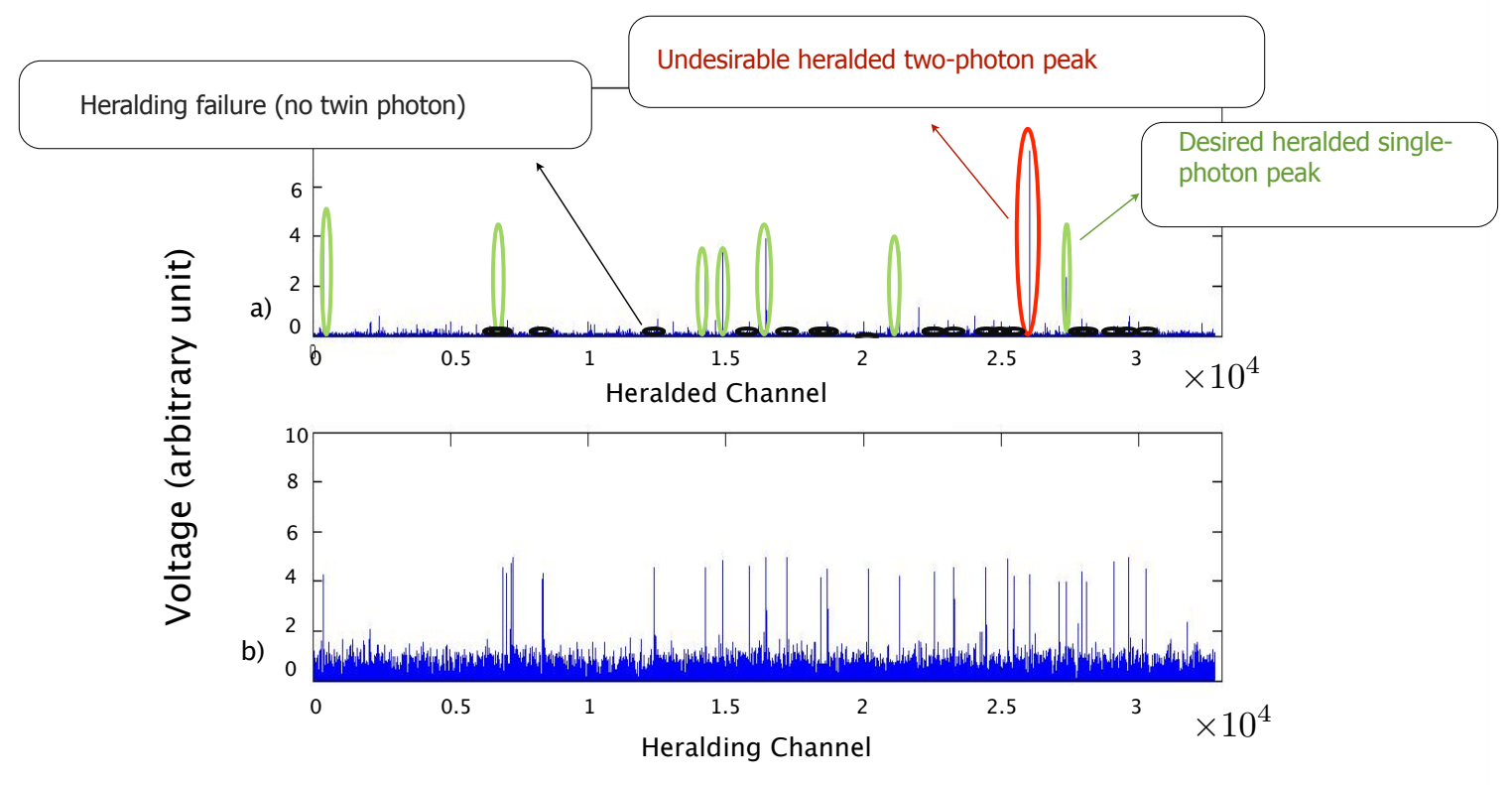

Number of sample points

Figure 4.4: TES data over 13 ms. a) heralded channel b) heralding channel, generated by disregarding higher photon peaks and keeping single photons. Red, green and black ellipses shows the contribution of two- photons $n_{2}$, single photons $n_{1}$ and no photons $n_{0}$ on the heralded channel after heralding on single photons on the heralding channel. total bin numbers on the heralded channel is $N_{b i n}=n_{0}+n_{1}+n_{2}$. 
second photon arrived while the TES is cooling down after the first photon, in this case second photon peak appeared on top of the decaying part of the first photon peak. As we discussed earlier in section 2.2, photon births at the rising edge and this second photon is independent and should not be combined with the first photon and be considered as a two photon peak, however increasing the number of sample points for photon sum then caused the pile up events to look like two photons and increased $n_{2}$ and the $g^{(2)}(0)$ as is clear from table 4.1 .

All $g^{(2)}(0)$ values for the heralded single photon source is less than 1, as presented

in table 4.1. $g^{(2)}(0)<1$ shows the quantum nature of our source. One can drop the pump power and properly design the detection window to achieve to $g^{(2)}(0)=0.03$.

\subsection{Interferometer Performance}

We studied the interferometer performance at a single-photon level, we first investigated the interferometer fed with bright and attenuated coherent state inputs to characterize the interferometer performance. Then, considered the interferometer with a single-photon input and calculated the PIE in reading classical information stored in Hadamard codings using a single photon, including all experimental imperfections that decrease the PIE from the predicted theoretical values.

\subsubsection{Interferometer with coherent state input}

To characterize the behavior of the interferometer at photon level, we fed it with attenuated laser beam and measured the output modes on the TES, we then compared 
its performance with the bright input interferometer. The intensity of the attenuated laser beam is in the fw level, which is equivalent to one photon per few $\mu s$.

The interferometer response is state independent and so all light constructively interfere at one output port and destructively on other ports at a constant Hadamard code, for both the single-photon and coherent state inputs. We sinusoidally scanned one of the interferometer mirrors to switch the interferometer phase between two Hadamard phase coded arrays, it caused photons to oscillate between det 1 and det 2 and resulted in an interference fringe on each of the dets 1,2 with respective $\pi$ phase shift, while det 3 and det 4 are always dark. Therefore, photons appeared only in one detector and the other detector is dark at each Hadamard phase. Fig. 4.5 compares fringes for the attenuated and bright coherent states respectively on the TES and regular power meters, in both cases the fringe visibility is in the order of $82 \pm 2 \%$. Each point on Fig. 4.5.b is integrated photon counts (number of photons arrived to detectors in $13 \mathrm{~ms}$ ) and is plotted versus the interferometer phase

\subsubsection{Interferometry with heralded single photon source}

After characterizing the TES with coherent state input, we sent a single-photon source to the interferometer. Heralded single-photon source is produced through the process of SPDC, as was explained in detail in section 2.3. Signal mode was directly sent to the TES detector and was used for the heralding and the the idler mode was sent to the interferometer. We then scanned the interferometer phase the same way as for the coherent state input and tried to reproduce similar fringes as Fig. 4.5. 


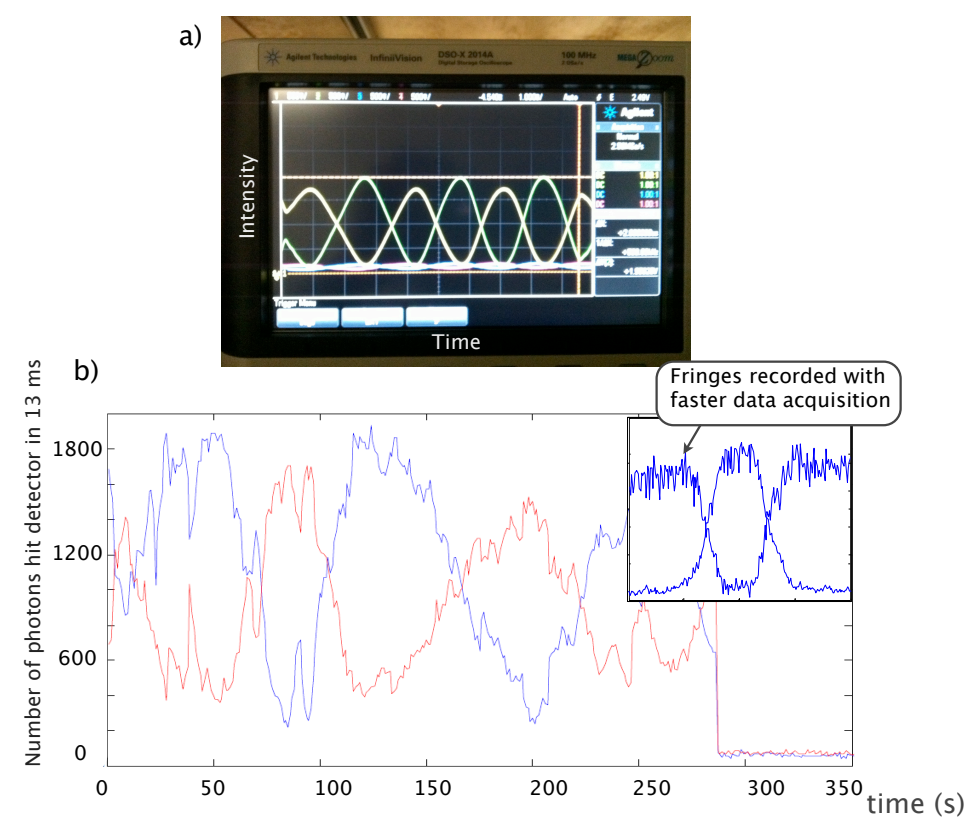

Figure 4.5: Interference fringes: a) bright coherent state input, recorded on the oscilloscope. b) attenuated seed beam where data is recorded on TES.

The interference fringe for a single-photon input is presented in Fig. 4.6, even though fringes are clear and intensity oscillation on two bright channels are appropriately out of phase, as expected, their interference visibility is not very high and is only $25 \%$ for one detector and $33 \%$ for the other one, which is much lower than the fringe visibility for coherent state input.

The cause for this low visibility is believed to be the extremely multimode (spatial and temporal) nature of SPDC emission.

Note that TES detector resolution was $1 \mu$ s and therefore the average flux of the source was less than 1 photon per 1 detection window. Therefore this was highly quantum interference wherein each photon went through all 4 paths of the interfer- 


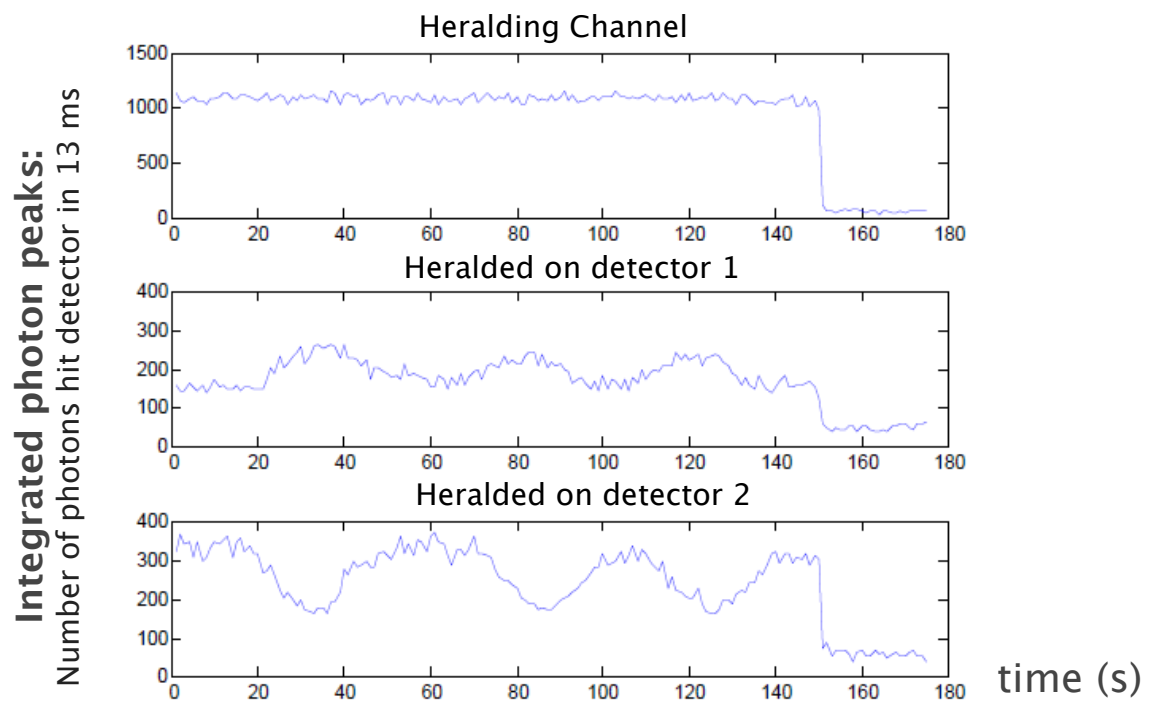

Figure 4.6: Interference fringes with single-photon input: integrated photon counts versus interferometer phase.

ometer and interfered only with itself.

An important remark must be brought up here: the interference fringes will be observed irrespective of the input state of the light, constructive interference being a classical wave phenomenon. It is only by evaluating the PIE that we will be able to ascertain how efficient a use we make of photon resources.

The PIE calculation depends crucially on the distinction between the number of sent, $n_{s}$, and received, $n_{r}$, photons. If we look at only the heralded photons received at the interferometer output we can expect a PIE of 2. However, if we account for the total photons sent in, including the ones which are lost in the interferometer or during the heralding process and gave us no information, then we have to account for these photons by multiplying the PIE by the percentage of heralding efficiency, i.e 
the number of photons we see on the heralded channel that are correlated with the heralding channel.

However, even for the $100 \%$ efficient heralded single-photon source, $n_{s}=n_{r}$, the fidelity and bandwidth of the source play an important role. In fact, in real experimental situation, photon will not interfere in the interferometer with $100 \%$ visibility which results in the possibility of photon appearing at wrong channel which corresponds to reading wrong information and so drops the PIE from maximum.

Therefore, in the perfect heralding experiment or even if one only wants to consider the received photon contribution for calculating the PIE, the information efficiency still very much depends on the so called interference fringes and cannot reach to the maximum value in imperfect interference of light in the interferometer.

In the following, we calculate the PIE value and its dependence to the fringe visibility.

\subsubsection{Photon Information Efficiency}

We have already studied the quantum optical reading of classical digital memory in section 3.6. The current experiment is another example of optical reading using quantum light to increase the rate of information read per photon. In this scheme as was first proposed by Saikat et al [72], the interferometer uses binary Hadamard code words to encode binary phases on $M$ pixels, each Hadamard code word consists M binary pixels, Fig. 4.1. So the channel capacity per pixel, Eq. (3.58), is: 


$$
C\left(N_{s}\right)=\frac{\max I(X ; Y)}{M}
$$

Where $I(X ; Y)=H(Y)-H(Y \mid X)$, Eq. (3.39), is the mutual information between the input X (which takes one of $M$ values - each value corresponding to a Hadamard codeword), and the output Y (which takes values for $M$ different click positions).

For an arbitrary quantum state with $\rho=\sum_{n} p_{n}|n\rangle\langle n|$, then the average photon number $\bar{N}=\sum_{n} n p_{n}$, the quantum state will pass through the MBS and then appear in $\mathrm{M}$ different paths, where each path contains $\frac{\bar{N}}{M}$ photons in average, so number of photons in each signal probe is $N_{s}=\frac{\bar{N}}{M}$. The PIE is then equal to the channel capacity per pixel per photon and can be calculated as:

$$
P I E=\frac{C\left(N_{s}\right)}{N_{s}}=\frac{\max I(X ; Y) / M}{\bar{N} / M}=\frac{\max I(X ; Y)}{\bar{N}}
$$

Therefore, assuming $p(x)=\frac{1}{M}$ for all $x$ and using Eq. 3.48, one can calculate PIE as:

$$
P I E=\frac{\log _{2} M-\frac{1}{M} \sum_{i, j} P\left(Y_{j} \mid X_{i}\right) \log _{2} \frac{P\left(Y_{j} \mid X_{i}\right)}{\sum_{k} P\left(Y_{j} \mid X_{k}\right)}}{\bar{N}}
$$

$$
P I E=\frac{\log _{2} M}{N} \text {, when } P\left(Y_{j} \mid X_{i}\right)_{i=j}=1 \text { and } P\left(Y_{j} \mid X_{i}\right)_{i \neq j}=0 \text {. }
$$

$P\left(Y_{j} \mid X_{i}\right)_{i=j}$, is the probability of success where a Hadamard code $H_{i}$ is applied and photon shows up at the bright channel $C h_{i}$, while $P\left(Y_{j} \mid X_{i}\right)_{i \neq j}$ is the probability of error, where a Hadamard code $H_{i}$ is applied but a photon appears at the dark channel 
say $C h_{j}$.

To calculate PIE for the $4 \times 4$ interferometer, $M=4$, we did look at the transition matrix $P(Y \mid X)$ for $\mathrm{X}\left(\right.$ Hadamard codes $\left.H_{i}\right)$ as rows and $\mathrm{Y}$ (click positions, $c h_{j}$ ) as columns :

$H_{1}\left(\begin{array}{cccc}0.38 & 0.21 & 0.21 & 0.21 \\ H_{2} & H_{4} \\ 0.22 & 0.35 & 0.22 & 0.22 \\ 0.2 & 0.2 & 0.4 & 0.2 \\ 0.21 & 0.21 & 0.21 & 0.37\end{array}\right)$

Therefore, substituting these probabilities in Eq. (4.5) results in:

$$
P I E=\frac{2-1.95}{\bar{N}}=\frac{0.05}{\bar{N}}
$$

This value for PIE is low and is due to very low interference visibility of SPDC modes.

We calculated the dependence of the PIE on the interference visibility. Assuming that all dark fringes have equal intensity and all four channels are similar, then the transition matrix can be written as: 


\begin{tabular}{|c|c|c|c|c|}
\hline & Ch 1 & Ch2 & Ch3 & Ch4 \\
\hline$H_{1}$ & $b$ & $d$ & $d$ & $d$ \\
\hline$H_{2}$ & $d$ & $b$ & $d$ & $d$ \\
\hline$H_{3}$ & $d$ & $d$ & $b$ & $d$ \\
\hline$H$ & $d$ & $d$ & $d$ & $b$ \\
\hline
\end{tabular}

Where $\mathrm{b}$ is the probability of photon ending up in bright channel versus $d$, the probability of reaching to dark channel for a constant Hadamard code. $3 d+b=1$. Interference fringe visibility can be written as

$$
v=\frac{b-d}{b+d}
$$

In Fig. 4.7, we plotted the dependence of PIE vs fringe visibility $v$. Low interference fringe visibilities will result in low PIE and in order to achieve the theoretical limits of PIE $=2$, the interference fringe visibility of close to $100 \%$ is required.

\subsection{Conclusion}

We studied an $M \times M$-port interferometer which is able to read $\log _{2}(M)$ classical bits of information using a single photon. We have successfully implemented a 4-port interferometer, which can distinguish between 4 Hadamard encoded phase arrays, and is an important step toward realization of the generalized $M \times M$-port interferometer.

We explained about the importance of the true single-photon source in this experiment, for attaining higher values of PIE, and showed that we can generate a heralded 


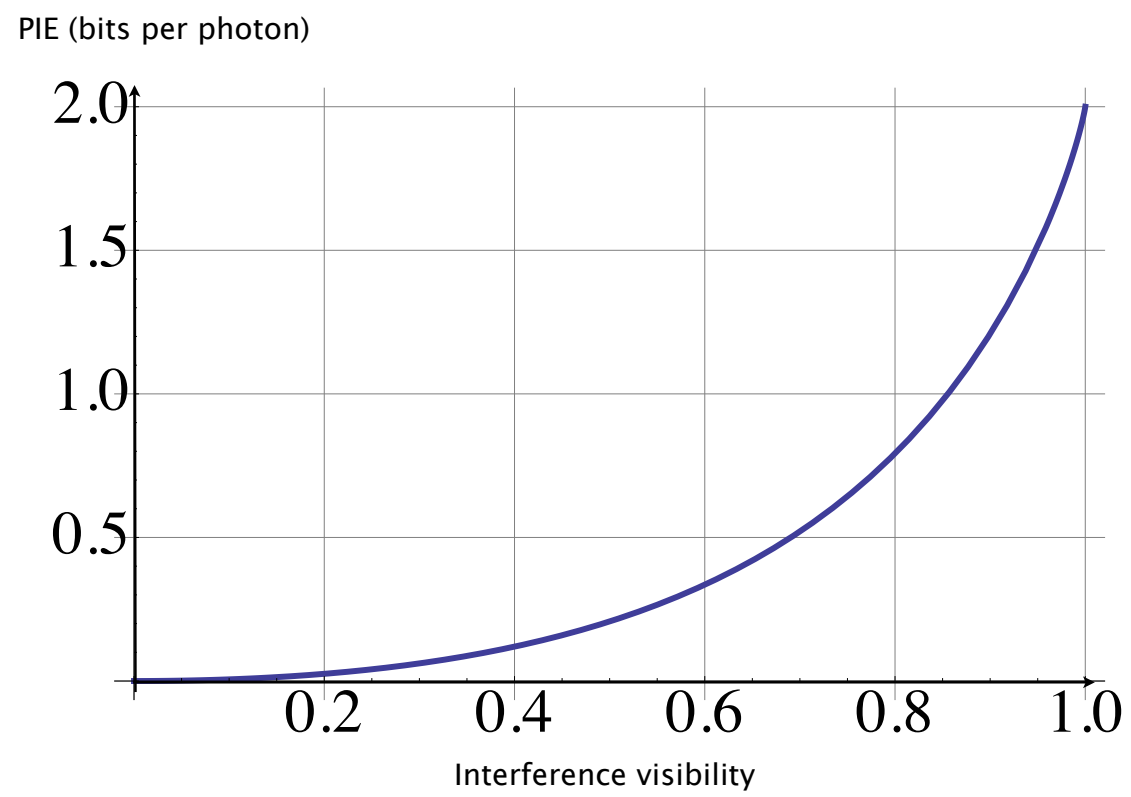

Figure 4.7: PIE vs interference visibility, lower interference visibilities will result in low PIE.

single-photon source with $g^{(2)}(0)$ values as low as 0.03 .

We then calculated the PIE $=0.05 \pm 0.01 \mathrm{bpp}$, and believed the difference between this low value for PIE and the theoretical predictions, $\mathrm{PIE}=2$, is due to the multimode nature of SPDC modes and this value can be improved by enhancing the quality of the heralded single photons. It inspired us to work harder to further improve the quality of our single-photon source by generating spatially and temporary well defined singlephoton sources as was discussed in section 2.4 . 


\section{Chapter 5}

\section{Conclusions}

This thesis presents significant progress toward experimental realization of spatially and temporally well-defined Fock-state source for applications in quantum information. The assumptions behind many quantum information protocols are based on Fock-state sources produced in single-mode and narrowband quantum states.

But the fidelity and the success rate of heralded Fock states, generated through the SPDC process, are limited by the multimode and broadband nature of SPDC emission. Therefore, we put extensive effort into generating single-mode and narrowband multi-photon source. We showed that using SPDC on PPKTP and HGKTP crystals followed by a narrowband Lorentzian filtering cavity cannot efficiently reduce the spectral and spatial bandwidth of the heralded single-photon source, it also significantly drops the heralding ratio. Therefore, we placed the nonlinear crystal inside an intrinsically stable Fabry-Perot cavity, the cavity enhancement effect boosts emission of SPDC photons into a well-defined cavity mode and generates high fidelity

photon pair. These well-defined photon pairs enabled us to herald single photons in 
narrowband $(\sim 10 \mathrm{MHz})$ and TEM00 quantum states, with up to $80 \%$ efficiency in preliminary results.

We believe that the experimental observation of cavity enhancement effect in increasing the heralding ratio and quality of single-photon source, paired with our efforts in improving data acquisition and analysis techniques will allow for the implementation of high quality Fock-state sources.

The heralding and measurements were performed by photon-number-resolving, high-quantum-efficiency, transition-edge-sensors (TES) built at NIST by Sae Woo Nam's group. These detectors are the essential requirement for efficient heralding on single-photons with low degree of second order coherence $g(2)(0)=0.03$. They are also required for heralding on Fock states with higher photon numbers.

We also suggested theoretical proposals in implementing Fock states for obtaining the quantum advantages in quantum information processing, here for error free M-ary optical phase discrimination and higher rate quantum optical reading of information stored in classical digital memory.

Our proposed experimental scheme is an MZI interferometer fed with twin Fock states, and is followed by a joint detection receiver. We showed that the interferometer can outperform optical reading techniques with coherent state inputs and direct detection receivers, as well as with Homodyne and Dolinar receivers.

As another example of implementing Fock states for advancing optical reading rates, we conducted an experimental effort to realize photon efficient quantum optical 
reading of classical digital memory. We built a $4 \times 4$ interferometer and injected a single photon into the interferometer, we showed that this experimental scheme is capable of reading 2 bits of information using a single photon, and is an important step toward realization of the generalized $M \times M$-port interferometer for reading $\log _{2} M$ bits of classical information, $\mathrm{M}$ can be arbitrary large and this scheme enables us to read boundless bits of information using a single photon. We then discussed the importance of the true single-photon source for obtaining higher photon information efficiencies.

We hope that our experimental efforts towards realization of high heralding ratio and high fidelity cavity-enhanced Fock-state generation can be paired with our theoretical proposals and improves quantum information protocols. 


\section{Appendix A}

\section{Large-scale multipartite entanglement in the optical frequency comb of a depleted-pump optical parametric oscillator}

In this appendix, I include my theoretical studies on the generation of multipartite entanglement on a massive scale in the spectrum, or optical frequency comb, of a single optical parametric oscillator (OPO) emitting well above threshold.

This work has been published in the journal of Quantum Information and Computation (QIC). 
Quantum Information and Computation, Vol. 12, No. 11\&12 (2012) 0953-0969

(C) Rinton Press

\title{
LARGE-SCALE MULTIPARTITE ENTANGLEMENT IN THE QUANTUM OPTICAL FREQUENCY COMB OF A DEPLETED-PUMP OPTICAL PARAMETRIC OSCILLATOR
}

\author{
REIHANEH SHAHROKHSHAHI and OLIVIER PFISTER \\ Department of Physics, University of Virginia \\ 382 McCormick Rd., Charlottesville, VA 22903, USA
}

Received October 29, 2011

Revised July 23, 2012

\begin{abstract}
We show theoretically that multipartite entanglement is generated on a massive scale in the spectrum, or optical frequency comb, of a single optical parametric oscillator (OPO) emitting well above threshold. In this system, the quantum dynamics of the strongly depleted pump field are responsible for the onset of the entanglement by correlating the two-mode squeezed, bipartite-entangled pairs of OPO signal fields. (Such pairs are independent of one another in the undepleted, classical pump approximation.) We verify the multipartite nature of the entanglement by evaluating the van Loock-Furusawa criterion for a particular set of entanglement witnesses deduced from physical considerations.

Keywords: Quantum information, quantum entanglement, continuous-variable entanglement

Communicated by: I Cirac \& E Polzik
\end{abstract}

\section{Introduction}

The generation of massively entangled states is of great importance for quantum information. For quantum communication, good examples are multiparty quantum teleportation [1] and quantum secret sharing [2]. For measurement-based quantum computing, cluster states [3, 4] are known to enable one-way quantum computing $[5,6]$. Constructing large-scale quantum registers and processors is therefore one of the prime objectives of experimental quantum information, along with the suppression or alleviation of decoherence.

In most cases, the approach to scaling up the size of quantum registers or processors is a "bottom-up" one, in which individual Qbits (following Mermin's more harmonious spelling [7]) are put together to form, say, an entangled quantum register [8]. Now, there are, indeed, extremely few examples of "top-down" approaches to multipartite entanglement, in which a single physical system enables intrinsic generation of multipartite entanglement over a large scale. To the best of our knowledge, there are but two such systems. The first one is the individually trapped atoms in an optical lattice initially loaded with a Bose-Einstein condensate subsequently undergoing a Mott insulator transition [9]. The second one is the ensemble of entangled quantum modes of light, a.k.a. "Qmodes," defined by the resonant frequencies - or quantum optical frequency comb (QOFC) - of an optical parametric oscillator (OPO), in which the QOFC is entangled by the OPO's nonlinear crystal [10, 11]. Recently, 
the simultaneous generation of 15 identical quadripartite "square" cluster states was realized experimentally over 60 Qmodes of a single OPO [12].

The QOFC entanglement experiments mentioned above necessitate an exquisitely sophisticated OPO [12], operated below threshold [13], and in which two or three different nonlinear interactions must be phasematched [14, 15].

In this paper, we present the theoretical discovery of massive multipartite entanglement generation in a much simpler system and in a completely different regime. The system is but a standard OPO, in which only one nonlinear interaction is phasematched. In addition, the OPO must be operated well above threshold. It is somewhat surprising that such a simple, well-known system might lend itself to the generation of such an exotic quantum state as a massively multipartite one. In particular, we emphasize that the entangling interaction is only pairwise. It is the fact that all entangled pairs are derived from the same, strongly depleted pump field that generates the multipartite entanglement by way of a bona fide 3field Hamiltonian. This is therefore a fundamentally different situation from that of the below-threshold OPO in which pairwise interactions are chained and all their pump fields are undepleted, yielding quadratic nonlinear interactions [10] in lieu of cubic ones.

This paper is organized as follows. In Section 2, we introduce the system Hamiltonian, distinguishing between the depleted and undepleted pump cases. In Section 3, we solve the equations of motion for the system by employing a linearization procedure. We are certainly aware that more sophisticated treatments exist $[16,17,18,19,13]$ and may indeed be interesting to use in order to explore this system further. In particular, it is worth mentioning the new physics of noncritical squeezing generation - that is, squeezing independent of the system parameters such as pump amplitude - in the transverse spatial modes of an OPO, which was recently predicted via the phenomena of spontaneous symmetry breaking $[20,21]$ and pump clamping [22], the latter having already been observed in the laboratory [23]. Also, in this novel regime, the well-known, laser-like (and usually slow) phase diffusion process of an OPO [24, 25] becomes entwined with the squeezed variables and affects detection [21], which isn't usually the case in a critically squeezing two-mode OPO [24, 25]. As the present paper doesn't pertain to noncritical squeezing, we have set aside this issue of phase diffusion for further studies, under the hypothesis that its effect may be similar to that in the usual critical squeezing situation. We therefore focus here on the nontrivial new results obtained from the simple approach adopted here. In Section 4, we use the multipartite inseparability criterion derived by van Loock and Furusawa [26] to establish the existence of multipartite entanglement in the optical frequency comb of a single OPO. We then conclude.

\section{The quantum optical frequency comb of a single OPO above threshold}

\subsection{Hamiltonian of the system}

We consider the simplest possible case of an OPO with a single, nondegenerate nonlinear interaction. In this case the interaction-picture Hamiltonian is

$$
H_{\text {int }}=2 i \hbar \chi \beta \sum_{i=1}^{n}\left(a_{i}^{\dagger} a_{-i}^{\dagger}-a_{i} a_{-i}\right)
$$

where $\beta$ is the classical (real) and constant (undepleted) pump field (in practice a stable, narrow-linewidth, continuous-wave laser) and $a_{ \pm i}$ are the photon annihilation operators of 
entangled Qmodes $\pm i$, of frequencies

$$
\omega_{ \pm i}=\frac{\omega_{p}}{2} \pm\left(i+\frac{1}{2}\right) \Delta
$$

where $\omega_{p}=\omega_{i}+\omega_{-i}$ is the pump frequency (see Fig. 1) and $\Delta$ is the free spectral range of

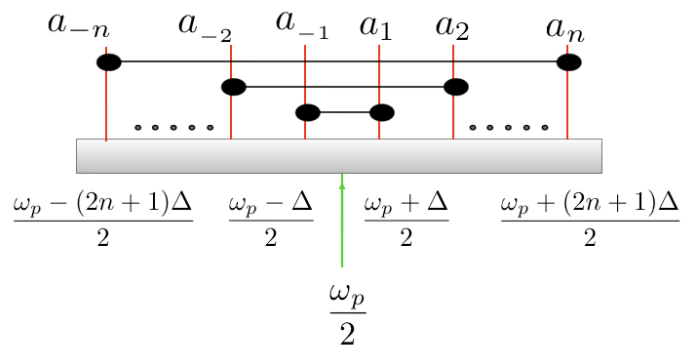

Fig. 1. Optical frequency comb defined by the resonant modes of the OPO cavity, spaced by free spectral range $\Delta$. The green arrow symbolizes the pump field, placed at half its frequency for clarity.

the OPO cavity.

Below the OPO emission threshold, such a system is known to emit two-mode squeezed fields which demonstrate the Einstein-Podolsky-Rosen (EPR) paradox [27, 28]. Above the OPO threshold, the undepleted classical pump approximation can still be taken to hold and the generation of EPR states has also been shown to be possible, theoretically [29] and experimentally $[30,31,32,33]^{a}$

However, the undepleted classical pump approximation breaks down if the external pump power is increased significantly above threshold. In that case, the pump must be treated as a quantum field in the three-wave mixing interaction

$$
H_{i n t}=2 i \hbar \chi \sum_{i=1}^{n}\left(p a_{i}^{\dagger} a_{-i}^{\dagger}-p^{\dagger} a_{i} a_{-i}\right)
$$

where $p$ is the annihilation operator of the pump field. Recently, it was predicted [34] and experimentally demonstrated [35] that the pump field participates in three-way entanglement in this case. Another interesting theoretical analysis showed that the signal fields from two OPOs pumped by the same field could become entangled [36]. Here, we extend this analysis to the QOFC of a single OPO, in which a vast number of different Qmode pairs are already known to be entangled by their parametric downconversion from the pump field [37, 12]. In the undepleted pump approximation, all EPR pumps are independent. However, when one considers the OPO well above threshold, there is but a single quantum pump field, whose strong (ideally total) depletion entail strong correlations between the EPR fields, since a pump photon downconverting into one Qmode pair will necessarily not be downconverted

\footnotetext{
${ }^{a}$ Note that all previous works featured the entanglement of a single Qmode pair at a time, which is not the situation described by Eq. (1). Indeed, Eq. (1) predicts many independent EPR pairs. Experimentally, this requires that the OPO cavity be resonant for all Qmode EPR pairs, which can be realized either by compensating birefringence in a type-II OPO or by using a type-I OPO. Dispersion is neglected in this discussion as its effects can be neglected for the first tens to hundreds of modes.
} 
into any other pair. This paper posits that this situation should yield multipartite, rather than bipartite, quantum correlations and our goal is to ascertain whether they result in multipartite entanglement, which they do.

\section{The quantum optical frequency comb of a single OPO above threshold}

As mentioned before, we consider the simplest possible case of an OPO cavity with a single pump mode and a single nondegenerate interaction in its nonlinear crystal. Such a crystal implements the Hamiltonian of Eq. (3). We assume a single two-mirror standing wave cavity, with one mirror of reflectivity $R_{ \pm i}^{\prime}=1$ for all modes and the other an output coupler of $R_{ \pm i}=1-T_{ \pm i}<1$. Taking into account the vacuum modes $A_{i}^{\text {in }}$ that enter the cavity through its output coupler, the input-output theory $[38,39]$ can be used to derive the equations of motion for the internal cavity modes

$$
\begin{aligned}
\dot{a}_{i} & =2 \chi p a_{-i}^{\dagger}-k_{i} a_{i}+\sqrt{2 k_{i}} A_{i}^{\mathrm{in}} \\
\dot{a}_{-i} & =2 \chi p a_{i}^{\dagger}-k_{-i} a_{-i}+\sqrt{2 k_{-i}} A_{-i}^{\mathrm{in}} \\
\dot{p} & =-2 \chi \sum_{i}^{n} a_{i} a_{-i}-k_{p} p+\sqrt{2 k_{p}} p_{i n} .
\end{aligned}
$$

Here $k_{ \pm i}=T_{ \pm i} / 2 \tau$ are the loss rates of the cavity mirror for mode $i, \tau$ being the cavity round trip time. In order to solve Eqs. (4-6) we first rewrite field operators as centered fluctuations about their expectation value

$$
\begin{aligned}
a_{i} & =\alpha_{i}+\delta a_{i} \\
A_{i}^{\text {in }} & =\delta A_{i}^{\text {in }} \\
p & =\varpi+\delta p \\
p_{i n} & =\varpi_{i n}+\delta p_{i n} .
\end{aligned}
$$

\subsection{Classical steady-state solutions}

The semiclassical, or mean value, equations follow directly from Eqs. (4-6):

$$
\begin{aligned}
\dot{\alpha}_{i} & =2 \chi \varpi \alpha_{-i}^{*}-k_{i} \alpha_{i} \\
\dot{\alpha}_{-i} & =2 \chi \varpi \alpha_{i}^{*}-k_{-i} \alpha_{-i} \\
\dot{\varpi} & =-2 \chi \sum_{i}^{n} \alpha_{i} \alpha_{-i}-k_{p} \varpi+\sqrt{2 k_{p}} \varpi_{i n} .
\end{aligned}
$$

Now, considering same cavity losses for all signal modes, $k_{i}=k_{-i}=k_{a}$, the stationary solutions of the two coupled equations Eqs. (11-12) for semi-classical mean values are

$$
\begin{aligned}
& 2 \chi \varpi \alpha_{-i}^{0 *}=k_{a} \alpha_{i}^{0} \\
& 2 \chi \varpi \alpha_{i}^{0 *}=k_{a} \alpha_{-i}^{0} .
\end{aligned}
$$

this results in $\left|\alpha_{i}^{0}\right|=\left|\alpha_{-i}^{0}\right|,\left|\varpi^{0}\right|=k_{a} / 2 \chi$ and $\phi_{i}^{0}+\phi_{-i}^{0}-\phi^{0}=0$ where $\phi_{ \pm i}^{0}$ and $\phi^{0}$ are the respective phases of $\alpha_{ \pm i}^{0}$ and $\varpi^{0}$. For simplicity we take $\varpi_{i n}$ real and positive therefore based 
on Eq. (13), $\phi^{0}=0$ and $\phi_{i}^{0}=-\phi_{-i}^{0}$. The stationary solution for the pump field's mean value can then be written as

$$
4 \chi^{2} \sum_{i}^{n}\left|\alpha_{i}^{0}\right|^{2}=k_{a} k_{p}(\sqrt{\sigma}-1)
$$

where

$$
\sigma=\left(\frac{2 \chi}{k_{a}} \sqrt{\frac{2}{k_{p}}} \varpi_{i n}\right)^{2} .
$$

Clearly, the right-hand side of Eq. (16) must be positive and $\sigma=1$ defines the threshold pump field

$$
\varpi_{i n}^{\mathrm{th}}=\frac{k_{a}}{2 \chi} \sqrt{\frac{k_{p}}{2}},
$$

Hence, $\sigma=\left(\varpi_{i n} / \varpi_{i n}^{\text {th }}\right)^{2}$ is also the pump to threshold power ratio. The classical signal amplitudes are weakly set by Eq. (16).

\subsection{Stability analysis}

The stability of the steady-state solution can be determined by a linearized analysis for small perturbations:

$$
\begin{aligned}
\alpha_{i} & =\alpha_{i}^{0}+\delta \alpha_{i} \\
\varpi_{i} & =\varpi_{i}^{0}+\delta \varpi_{i} .
\end{aligned}
$$

Substituting Eqs. (19-20) into Eqs. (11-13), we get

$$
\begin{aligned}
\delta \dot{\alpha}_{i} & =k_{a} \delta \alpha_{-i}^{*}+2 \chi \alpha_{i}^{0} \delta \varpi-k_{a} \delta \alpha_{i} \\
\delta \alpha_{-i}^{\cdot} & =k_{a} \delta \alpha_{i}^{*}+2 \chi \alpha_{-i}^{0} \delta \varpi-k_{a} \delta \alpha_{-i} \\
& \vdots \\
\delta \dot{\varpi} & =-2 \chi \sum_{i}^{n}\left(\alpha_{i}^{0} \delta \alpha_{-i}+\alpha_{-i}^{0} \delta \alpha_{i}\right)-k_{p} \delta \varpi
\end{aligned}
$$

where $i=1, \ldots, n, n$ being the number of signal and idler pairs considered inside the cavity. Defining $\delta A=\left(\begin{array}{llllllll}\ldots & \delta \alpha_{i} & \delta \alpha_{i}^{*} & \delta \alpha_{-i} & \delta \alpha_{-i}^{*} & \ldots & \delta \varpi & \delta \varpi^{*}\end{array}\right)^{T}$ We can rewrite Eqs. (21-23) in block matrix form:

$\frac{d}{d t}\left(\begin{array}{c}\vdots \\ \delta \alpha_{i} \\ \delta \alpha_{i}^{*} \\ \delta \alpha_{-i} \\ \delta \alpha_{-i}^{*} \\ \vdots \\ \delta \varpi \\ \delta \varpi^{*}\end{array}\right)=\left(\begin{array}{cccccccc} & \vdots & \vdots & \vdots & \vdots & & \vdots & \vdots \\ \ldots & -k_{a} & 0 & 0 & k_{a} & \ldots & 2 \chi \alpha_{i}^{0} & 0 \\ \ldots & 0 & -k_{a} & k_{a} & 0 & \ldots & 0 & 2 \chi \alpha_{i}^{0 *} \\ \ldots & 0 & k_{a} & -k_{a} & 0 & \ldots & 2 \chi \alpha_{-i}^{0} & 0 \\ \ldots & k_{a} & 0 & 0 & -k_{a} & \ldots & 0 & 2 \chi \alpha_{-i}^{0 *} \\ & \vdots & \vdots & \vdots & \vdots & & \vdots & \vdots \\ \ldots & -2 \chi \alpha_{-i}^{0} & 0 & -2 \chi \alpha_{i}^{0} & 0 & \ldots & -k_{p} & 0 \\ \ldots & 0 & -2 \chi \alpha_{-i}^{0 *} & 0 & -2 \chi \alpha_{i}^{0 *} & \ldots & 0 & -k_{p}\end{array}\right)\left(\begin{array}{c}\vdots \\ \delta \alpha_{i} \\ \delta \alpha_{i}^{*} \\ \delta \alpha_{-i} \\ \delta \alpha_{-i}^{*} \\ \vdots \\ \delta \varpi \\ \delta \varpi^{*}\end{array}\right)$. 
We derived the eigenvalues of the matrix in Eq. (24) for $n=1,2,3$. In all these cases, the eigenvalue sets have the following form:

$$
\{\lambda\}=\{\underbrace{0, \ldots, 0}_{2 n-1}, \underbrace{-2 k_{a}, \ldots,-2 k_{a}}_{2 n-1}, \lambda_{1}, \lambda_{2}, \lambda_{3}, \lambda_{4}\}
$$

where, posing the pump-signal loss ratio $\kappa=k_{p} / k_{a}$,

$$
\begin{aligned}
& \lambda_{1,2}=-\frac{1}{2} k_{a}(\kappa \pm \sqrt{\kappa[\kappa-8(\sqrt{\sigma}-1)]}) \\
& \lambda_{3,4}=-\frac{1}{2} k_{a}\left(\kappa+2 \pm \sqrt{(\kappa+2)^{2}-8 n \sqrt{\sigma}}\right) .
\end{aligned}
$$

Because of the particular symmetry of the problem - namely the block structure of the matrix in Eq. (24), we argue that it is reasonable to postulate that Eq. (25) is the general eigenvalue set, $\forall n$, even though a complete inductive proof is formally required. For certain initial conditions all $2(2 n+1)$ eigenvalues of the matrix in Eq. (25) can only be zero or negative, which ensures the stability of the stationary solution presented in Eq. (16). Equations (26-27) show that, as the number of times above threshold $\sigma$ increases, one can always find negative values for $\lambda_{1, \ldots, 4}$ by increasing the pump-signal loss ratio $\kappa$, thereby tending towards the doubly resonant OPO, which is always stable.

\subsection{Quantum fluctuations}

Now, we rewrite Eqs. (4-6) for the quantum fluctuations around these classical mean values. Notice $\alpha_{-i}^{*}=\alpha_{i}$,

$$
\begin{aligned}
\dot{\delta \dot{a}_{i}} & =2 \chi\left(\delta p \alpha_{i} e^{i \phi_{i}}+\varpi \delta a_{-i}^{\dagger}\right)-k_{a} \delta a_{i}+\sqrt{2 k_{a}} \delta A_{i}^{\mathrm{in}} \\
\delta \dot{a}_{-i} & =2 \chi\left(\delta p \alpha_{i} e^{-i \phi_{i}}+\varpi \delta a_{i}^{\dagger}\right)-k_{a} \delta a_{-i}+\sqrt{2 k_{a}} \delta A_{-i}^{\mathrm{in}} \\
\delta \dot{p} & =-2 \chi \sum_{i}^{n}\left(\alpha_{i} e^{i \phi_{i}} \delta a_{-i}+\alpha_{i} e^{-i \phi_{i}} \delta a_{i}\right)-k_{p} \delta p+\sqrt{2 k_{p}} \delta p_{i n} .
\end{aligned}
$$

We introduce the generalized field quadrature operators as $Q_{i}=\left(e^{i \phi_{i}} a_{i}^{\dagger}+e^{-i \phi_{i}} a_{i}\right)$ and $P_{i}=$ $i\left(e^{i \phi_{i}} a_{i}^{\dagger}-e^{-i \phi_{i}} a_{i}\right)$. Then solve these coupled equations we can use the symmetry of the equations in the exchange of the two signal modes and introduce the new variables [40]

$$
\begin{aligned}
Q_{i+} & =Q_{i}+Q_{-i} \\
Q_{i-} & =Q_{i}-Q_{-i} \\
P_{i+} & =P_{i}+P_{-i} \\
P_{i-} & =P_{i}-P_{-i} .
\end{aligned}
$$


The equations of motion for these quadratures are

$$
\begin{aligned}
\delta \dot{Q}_{i+} & =4 \chi \alpha_{i} \delta Q_{p}+\sqrt{2 k_{a}} \delta Q_{i+}^{\mathrm{in}} \\
\delta \dot{Q}_{i-} & =-2 k_{a} \delta Q_{i-}+\sqrt{2 k_{a}} \delta Q_{i-}^{\mathrm{in}} \\
\delta \dot{Q}_{p} & =-2 \chi \sum_{i}^{n} \alpha_{i} \delta Q_{i+}-k_{p} \delta Q_{p}+\sqrt{2 k_{p}} \delta Q_{p}^{\mathrm{in}} \\
\delta \dot{P}_{i+} & =4 \chi \alpha_{i} \delta P_{p}-2 k_{a} \delta P_{i+}+\sqrt{2 k_{a}} \delta P_{i+}^{\mathrm{in}} \\
\delta \dot{P}_{i-} & =\sqrt{2 k_{a}} \delta P_{i-}^{\text {in }} \\
\delta \dot{P}_{p} & =-2 \chi \sum_{i}^{n} \alpha_{i} \delta P_{i+}-k_{p} \delta P_{p}+\sqrt{2 k_{p}} \delta P_{p}^{\text {in }} .
\end{aligned}
$$

As seen from Eq. (36) and Eq. (39), the equations for the antisymmetric modes are decoupled from the pump and the solutions are, in the frequency domain [40],

$$
\begin{aligned}
& \delta \tilde{Q}_{-i}^{\text {out }}(\Omega)=-\frac{i \Omega}{2 k_{a}+i \Omega} \delta \tilde{Q}_{-i}^{\text {in }}(\Omega) \\
& \delta \tilde{P}_{-i}^{\text {out }}(\Omega)=\left(-1-\frac{2 i k_{a}}{\Omega}\right) \delta \tilde{P}_{-i}^{\text {in }}(\Omega) .
\end{aligned}
$$

The frequency-domain equations for the symmetric modes are [40]

$$
\begin{aligned}
& i \Omega \delta \tilde{Q}_{i+}(\Omega)=4 \chi \alpha_{i} \delta \tilde{Q}_{p}(\Omega)+\sqrt{2 k_{a}} \delta \tilde{Q}_{i+}^{\text {in }}(\Omega) \\
& i \Omega \delta \tilde{P}_{i+}(\Omega)=4 \chi \alpha_{i} \delta \tilde{P}_{p}(\Omega)-2 k_{a} \delta \tilde{P}_{i+}(\Omega)+\sqrt{2 k_{a}} \delta \tilde{P}_{i+}^{\text {in }}(\Omega) \\
& i \Omega \delta \tilde{Q}_{p}(\Omega)=-2 \chi \sum_{i}^{n} \alpha_{i} \delta \tilde{Q}_{i+}(\Omega)-k_{p} \delta \tilde{Q}_{p}(\Omega)+\sqrt{2 k_{p}} \delta \tilde{Q}_{p}^{\text {in }}(\Omega) \\
& i \Omega \delta \tilde{P}_{p}(\Omega)=-2 \chi \sum_{i}^{n} \alpha_{i} \delta \tilde{P}_{i+}(\Omega)-k_{p} \delta \tilde{P}_{p}(\Omega)+\sqrt{2 k_{p}} \delta \tilde{P}_{p}^{\text {in }}(\Omega) .
\end{aligned}
$$

These equations can be easily solved for pump and signal- idler pairs. The output quadratures are finally determined using input-output relations:

$$
\begin{aligned}
& \delta Q_{ \pm}^{\text {out }}=\sqrt{2 k_{a}} \delta Q_{ \pm}-\delta Q_{ \pm}^{\text {in }} \\
& \delta P_{ \pm}^{\text {out }}=\sqrt{2 k_{a}} \delta P_{ \pm}-\delta P_{ \pm}^{\text {in }} \\
& \delta Q_{p}^{\text {out }}=\sqrt{2 k_{p}} \delta Q_{p}-\delta Q_{p}^{\text {in }} \\
& \delta P_{p}^{\text {out }}=\sqrt{2 k_{p}} \delta P_{p}-\delta P_{p}^{\text {in }} .
\end{aligned}
$$


The solutions of Eqs. (43-46) are

$$
\begin{aligned}
& \delta \tilde{Q}_{+i}^{\text {out }}(\Omega)=-\left(1+\frac{2 k_{a}\left\{k_{p} \Omega+i\left[\Omega^{2}+8 \chi^{2}\left(\alpha_{i}^{2}-\sum_{j} \alpha_{j}^{2}\right)\right]\right\}}{\Omega\left(-i k_{p} \Omega+\Omega^{2}-8 \chi^{2} \sum_{j} \alpha_{j}^{2}\right)}\right) \delta \tilde{Q}_{+, i}^{\text {in }}(\Omega) \\
& -\frac{16 i \chi^{2} k_{a} \alpha_{i}}{\Omega\left(-i k_{p} \Omega+\Omega^{2}-8 \chi^{2} \sum_{j} \alpha_{j}^{2}\right)} \sum_{j \neq i} \alpha_{j} \delta \tilde{Q}_{+, j}^{\text {in }}(\Omega) \\
& -\frac{8 \chi \sqrt{k_{a} k_{p}} \alpha_{i}}{-i k_{p} \Omega+\Omega^{2}-8 \chi^{2} \sum_{j} \alpha_{j}^{2}} \delta \tilde{Q}_{p}^{\text {in }}(\Omega) \\
& \delta \tilde{P}_{+i}^{\text {out }}(\Omega)=\left(-1+\frac{2 k_{a}}{2 k_{a}+i \Omega}-\frac{16 \chi^{2} k_{a} \alpha_{i}^{2}}{\left(2 k_{a}+i \Omega\right)\left[\left(2 k_{a}+i \Omega\right)\left(k_{p}+i \Omega\right)+8 \chi^{2} \sum_{j} \alpha_{j}^{2}\right]}\right) \delta \tilde{P}_{+, i}^{\text {in }}(\Omega) \\
& -\frac{16 \chi^{2} k_{a} \alpha_{i}}{\left(2 k_{a}+i \Omega\right)\left[\left(2 k_{a}+i \Omega\right)\left(k_{p}+i \Omega\right)+8 \chi^{2} \sum_{j} \alpha_{j}^{2}\right]} \sum_{j \neq i} \alpha_{j} \delta \tilde{P}_{+, j}^{\text {in }}(\Omega) \\
& +\frac{8 \chi \sqrt{k_{a} k_{p}} \alpha_{i}}{\left(2 k_{a}+i \Omega\right)\left(k_{p}+i \Omega\right)+8 \chi^{2} \sum_{j} \alpha_{j}^{2}} \delta \tilde{P}_{p}^{\text {in }}(\Omega) \\
& \delta \tilde{Q}_{p}^{\text {out }}(\Omega)=\frac{k_{p} \Omega-i\left(\Omega^{2}-8 \chi^{2} \sum_{j} \alpha_{j}^{2}\right)}{k_{p} \Omega+i\left(\Omega^{2}-8 \chi^{2} \sum_{j} \alpha_{j}^{2}\right)} \delta \tilde{Q}_{p}^{\text {in }}(\Omega) \\
& +\frac{4 i \chi \sqrt{k_{a} k_{p}}}{k_{p} \Omega+i\left(\Omega^{2}-8 k^{2} \sum_{j} \alpha_{j}^{2}\right)} \sum_{j=1}^{n} \alpha_{j} \delta \tilde{Q}_{+, j}^{\mathrm{in}}(\Omega) \\
& \delta \tilde{P}_{p}^{\text {out }}(\Omega)=\frac{2 k_{a}\left(k_{p}-i \Omega\right)+i k_{p} \Omega+\Omega^{2}-8 \chi^{2} \sum_{j} \alpha_{j}^{2}}{2 k_{a}\left(k_{p}+i \Omega\right)+i k_{p} \Omega-\Omega^{2}+8 k^{2} \sum_{j} \alpha_{j}^{2}} \delta \tilde{P}_{p}^{\text {in }}(\Omega) \\
& -\frac{4 k \sqrt{k_{a}} \sqrt{k_{p}}}{2 k_{a}\left(k_{p}+i \Omega\right)+i k_{p} \Omega-\Omega^{2}+8 k^{2} \sum_{j} \alpha_{j}^{2}} \sum_{i=1}^{n} \alpha_{i} \delta \tilde{P}_{+, i}^{\text {in }}(\Omega) .
\end{aligned}
$$

Substituting the classical solutions Eq. (16) in Eqs. (48-51) and taking $\Omega=0$, these equations yield:

$$
\begin{aligned}
& \delta Q_{-i}^{\text {out }} \longrightarrow 0 \\
& \delta P_{-i}^{\text {out }} \longrightarrow \infty \\
& \delta Q_{+i}^{\text {out }} \longrightarrow \infty \\
& \delta P_{+i}^{\text {out }}=\frac{4 \chi \sqrt{k_{a} k_{p}} \alpha_{i}}{k_{a} k_{p} \sqrt{\sigma}} \delta P_{p}^{\text {in }}-\frac{4 \chi^{2} \alpha_{i}^{2}}{k_{a} k_{p} \sqrt{\sigma}} \delta P_{+, i}^{\text {in }}-\frac{4 \chi^{2} \alpha_{i}}{k_{a} k_{p} \sqrt{\sigma}} \sum_{j \neq i} \alpha_{j} \delta P_{+j}^{\text {in }}
\end{aligned}
$$

\subsubsection{Two-mode squeezing}

In order to quantitatively study squeezing behavior, we assumed equal classical mean values for all pairs. However, we can assume any ratio between the classical mean values, as long 
as they satisfy $\sum_{i}^{n}\left|\alpha_{i}\right|^{2}=\frac{k_{a} k_{p}(\sqrt{\sigma}-1)}{4 \chi^{2}}=$ const, Eq. (16). Therefore the variances of squeezed and antisqueezed quadratures are

$$
\begin{aligned}
V\left(Q_{-i}\right) & =\left\langle\left(\delta Q_{-i}\right)^{2}\right\rangle \longrightarrow 0 \\
V\left(P_{-i}\right) & =\left\langle\left(\delta P_{-i}\right)^{2}\right\rangle \longrightarrow \infty \\
V\left(Q_{+i}\right) & =\left\langle\left(\delta Q_{+i}\right)^{2}\right\rangle \longrightarrow \infty \\
V\left(P_{+i}\right) & =\left\langle\left(\delta P_{+i}\right)^{2}\right\rangle=\frac{2(\sigma-1)}{n \sigma},
\end{aligned}
$$

which yields the classic EPR result at threshold $(\sigma=1)$, the generation of $n$ independent entangled $(i,-i)$ pairs.

However, if the OPO is above threshold $(\sigma>1)$, the variance of the phase sum, Eq. (59) will increase from zero [40,29] and will eventually stop being squeezed. It states the wellknown fact that for the OPO operating well above the emission threshold, twin pairs are not independent EPR pairs due to the pump statistics, unless $k_{p} \ll k_{a}$ [29]. This is precisely the mechanism that we rely upon to create multipartite entanglement in this work. We give two preliminary examples before turning to the evaluation of precise multimode entanglement criteria.

\subsubsection{Multimode squeezing}

We give two examples of squeezed multimode operators which will be useful in the next section.

First off, specifically combining phase sum operators of two pairs $i$ and $j$ yields

$$
\alpha_{i}\left(P_{j}+P_{-j}\right)-\alpha_{j}\left(P_{i}+P_{-i}\right) \longrightarrow 0 .
$$

Even though phase sum operators for each pair become noisier with increasing input pump, this noise can be canceled appropriate linear combinations.

Another interesting example is that of operator $\sum_{i=1}^{n}\left(P_{i}+P_{-i}\right)-x P_{p}$. In Fig. 2 we plot

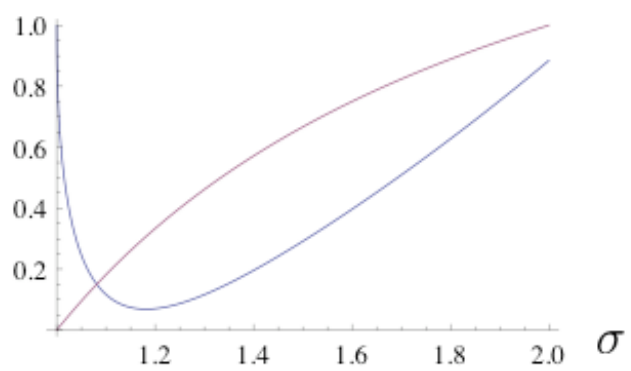

Fig. 2. Plot of $n V\left(P_{i}+P_{-i}\right)$, in red, and $V\left[\sum_{i=1}^{n}\left(P_{i}+P_{-i}\right)-x P_{p}\right]$, in blue, for $n=3$ and $x=\sigma$. When $\sigma$ increases, the variance of $P_{i}+P_{-i}$ increases from zero and approaches the shot noise level. However, the variance of $\sum_{i=1}^{n}\left(P_{i}+P_{-i}\right)-x P_{p}$, not squeezed at threshold, subsequently drops from the shot noise level (of value 1 in this graph) and shows squeezing. In this particular graph, the minimum of $V\left[\sum_{i=1}^{n}\left(P_{i}+P_{-i}\right)-x P_{p}\right]$ occurs at $\sigma=1.18$ but, in general, the value of $\sigma$ for which the blue curve reaches its minimum, as well as the value of the minimum itself, depends on the choice of $x$. 
its variance as a function of $\sigma$, for $x=\sigma$. This graph clearly shows that the assumption of the existence of a correlation between all modes and the pump is a sensible one. Having established this, we turn to directly testing the existence of multipartite entanglement in our system.

\section{Multipartite entanglement in the OPO well above threshold}

\subsection{The van Loock-Furusawa inseparability criterion}

The van Loock-Furusawa (vLF) multipartite entanglement criterion [26] is the multipartite generalization of the Duan [41]-Simon [42] criterion, itself the continuous-variable formulation of the Peres [43]-Horodecki [44] positive partial transpose criterion. A density operator is partially separable if and only if it can be written as the convex sum

$$
\hat{\rho}=\sum_{i} \eta_{i} \hat{\rho}_{i, k_{1} \ldots \ldots k_{m}} \otimes \hat{\rho}_{i, k_{m+1} \ldots, k_{n}}
$$

where the mode set $\left(k_{1}, \ldots, k_{m}\right)$ is separable from the mode set $\left(k_{m+1}, \ldots, k_{n}\right)$. If we define two "entanglement witnesses," quadrature operators with arbitrary real parameter sets $\left\{h_{i}\right\}_{i}$ and $\left\{g_{i}\right\}_{i}$

$$
\begin{array}{r}
u=h_{1} Q_{1}+h_{2} Q_{2}+\cdots+h_{n} Q_{n} \\
v=g_{1} P_{1}+g_{2} P_{2}+\cdots+g_{n} P_{n},
\end{array}
$$

then the separable density operator of Eq. (61) must verify the vLF inequality [26]

$$
V_{\rho}(u)+V_{\rho}(v) \geqslant 2\left(\left|h_{k_{1}} g_{k_{1}}+\cdots+h_{k_{m}} g_{k_{m}}\right|+\left|h_{k_{m+1}} g_{k_{m+1}}+\cdots+h_{k_{n}} g_{k_{n}}\right|\right)
$$

whose violation implies the existence of entanglement between mode set $\left(k_{r}, \ldots, k_{m}\right)$ and mode set $\left(k_{s}, \ldots, k_{n}\right)$. Operators $u$ and $v$ were coined variance-based entanglement witnesses for continuous-variable systems by Hyllus and Eisert [45], in reference to the original Qbit expectation-value-based entanglement witnesses [44, 46, 47].

\subsection{Multipartite entanglement in a single, depleted-pump OPO}

In order to demonstrate multipartite entanglement, we examine the conditions for violation of all possible vLF inequalities, corresponding to all possible respective mode partitions such as Eq. (61), and their associated experimental regimes.

\subsubsection{Pump-signals partition}

We first consider the separability of the sole pump mode from all signal modes. We define $u_{1}$ and $v_{1}$ as

$$
\begin{aligned}
& u_{1}=\sum_{i=1}^{n} \frac{\alpha_{i}}{\alpha_{1}}\left(Q_{i}+Q_{-i}\right)+\frac{2}{x} \sum_{i=1}^{n} \frac{\alpha_{i}}{\alpha_{1}} Q_{p} \\
& v_{1}=\sum_{i=1}^{n}\left(P_{i}+P_{-i}\right)-x P_{p}
\end{aligned}
$$


with the real parameter $x>0$. Based on Eq. (64), the separability of mode $p$ implies

$$
\begin{aligned}
S_{1}=V\left(u_{1}\right)+V\left(v_{1}\right) & \geqslant 2\left(\left|h_{-n} g_{-n}+\cdots+h_{-1} g_{-1}+h_{1} g_{1}+\cdots+h_{n} g_{n}\right|+\left|h_{p} g_{p}\right|\right) \\
& \geqslant 2\left(2\left|h_{1} g_{1}+\cdots+h_{n} g_{n}\right|+\left|h_{p} g_{p}\right|\right) \\
& \geqslant 2\left(\left|\frac{2}{\alpha_{1}} \sum_{i=1}^{n} \alpha_{i}\right|+\left|\frac{2}{x \alpha_{1}} \sum_{i=1}^{n} \alpha_{i}(-x)\right|\right) \\
& \geqslant \frac{8}{\alpha_{1}} \sum_{i=1}^{n} \alpha_{i} .
\end{aligned}
$$

Here, and in the following, we make the assumption that all classical amplitudes $\left\{\alpha_{i}\right\}_{i}$ are equal, for the sake of simplicity. This doesn't lessen the generality of our treatment and makes numerical evaluations easier. Under this assumption, we get

$$
S_{1} \geqslant 8 n \text {. }
$$

Figure 3 displays the maximum violation of the above inequality versus $n$ and $\sigma$, for optimized values of the arbitrary weight $x$. As can be seen, there always exist values of $(n, \sigma)$ for which $S_{1}-8 n$ is negative, which proves the inseparability of the pump mode from the signal modes. Unsurprisingly, entangling a larger number of pairs $(n)$ requires a higher pump to threshold power ratio $(\sigma)$. However, further increasing the pump power $(\sigma)$ does degrade the inseparability, which might just be due to the increasing depletion of the intracavity pump field.
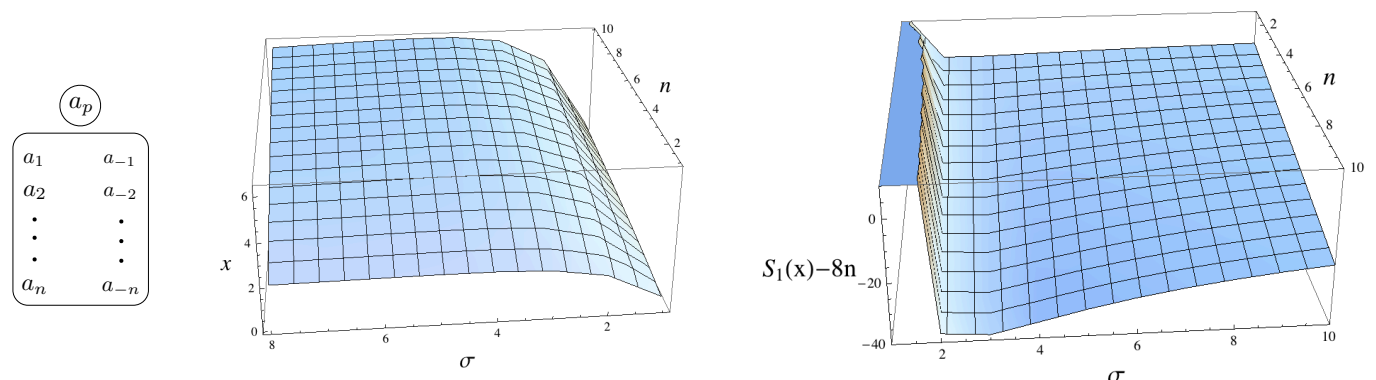

Fig. 3. Left, sketch of the mode partition studied. Center, plot of the optimum values of $x=x_{\mathrm{opt}}$ which give maximum violation $S_{1}(x)-8 n$ of the $\mathrm{vLF}$ inequality, at a given pump to threshold power ratio $\sigma$ and a given number $n$ of mode pairs inside the cavity. Right, plot of the maximum vLF inequality violation $S_{1}\left(x_{\mathrm{opt}}\right)-8 n$, versus $\sigma$ and $n$. We took the particular case $\Omega=0$ and $\alpha_{i}=\alpha_{j} \forall i, j$.

\subsubsection{Partition of one $\left(a_{j}, a_{-j}\right)$ EPR pair}

We now study the inseparability of an entangled pair $\left(a_{j}, a_{-j}\right)$ from the rest of the signals and the pump. For such a partition, we define

$$
\begin{aligned}
& u_{2}=Q_{j+}+\sum_{i \neq j}^{n} \frac{\alpha_{i}}{\alpha_{j}} Q_{i+}+\frac{2}{x \alpha_{j}} \sum_{i}^{n} \alpha_{i} Q_{p} \\
& v_{2}=\sum_{i \neq j}^{n}\left(\frac{\alpha_{i}}{\alpha_{j}} P_{j+}-P_{i+}\right),
\end{aligned}
$$


and the vLF inequality is

$$
\begin{aligned}
S_{2}=V\left(u_{2}\right)+V\left(v_{2}\right) & \geqslant 2\left(\left|2 h_{j} g_{j}\right|+\left|2 h_{1} g_{1}+\cdots+2 h_{n} g_{n}+h_{p} g_{p}\right|\right) \\
& \geqslant 2\left|\left(2 \frac{1}{\alpha_{j}} \sum_{i \neq j}^{n} \alpha_{i}\right)\right|+2\left|-\frac{2}{\alpha_{j}} \sum_{i \neq}^{n} \alpha_{i}+0\right| \\
& \geqslant \frac{8}{\alpha_{j}} \sum_{i \neq j}^{n} \alpha_{i} \\
& \geqslant 8(n-1) .
\end{aligned}
$$

Figure 4 shows the violation of this inequality for a broad range of parameters. It also demonstrates the necessity of applying larger input pump intensity when considering more pairs inside the cavity in order to generate inseparability between all pairs, again unsurprisingly.
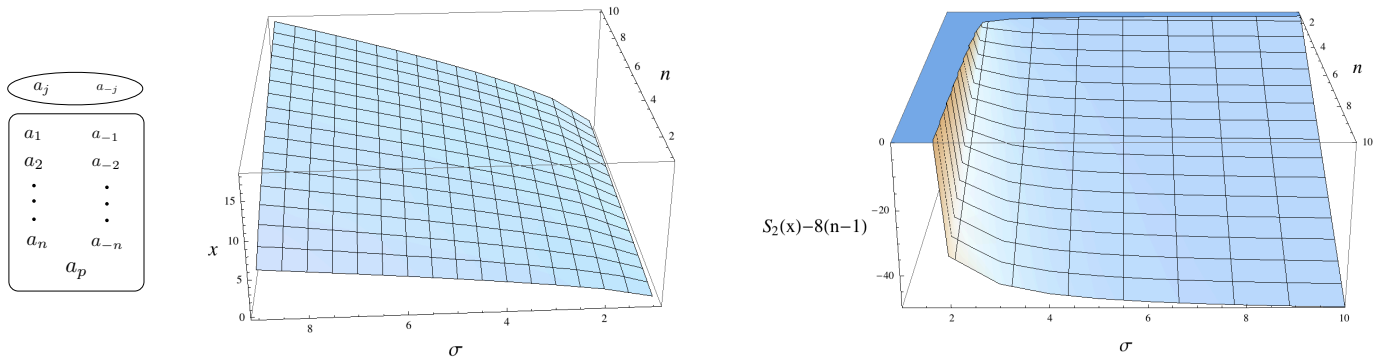

Fig. 4. Left, sketch of the mode partition studied. Center, plot of the optimum values of $x=x_{\mathrm{opt}}$ which give maximum violation $S_{2}(x)-8(n-1)$ of the $\mathrm{vLF}$ inequality, at a given pump to threshold power ratio $\sigma$ and a given number $n$ of mode pairs inside the cavity. Right, plot of the maximum vLF inequality violation $S_{2}\left(x_{\mathrm{opt}}\right)-8(n-1)$, versus $\sigma$ and $n$. We took the particular case $\Omega=0$ and $\alpha_{i}=\alpha_{j} \forall i, j$.

\subsubsection{Pair set partition}

We next turn to partitions $\left\{\left(a_{1}, b_{1}\right) \ldots\left(a_{k}, b_{k}\right)\right\}\left\{\left(a_{k+1}, b_{k+1}\right) \ldots\left(a_{n}, b_{n}\right)\right\}$. The operators are

$$
\begin{aligned}
& u_{3}=\sum_{i=1}^{k} Q_{i+}+\sum_{j=k+1}^{n} \frac{\alpha_{j}}{\alpha_{1}} Q_{j+}+\frac{2}{x \alpha_{1}} \sum_{l}^{n} \alpha_{l} Q_{p} \\
& v_{3}=\sum_{i=1}^{k} \sum_{j \neq i}^{n}\left(P_{i+}-\frac{\alpha_{i}}{\alpha_{j}} P_{j+}\right),
\end{aligned}
$$

and the vLF inequality is

$$
\begin{aligned}
S_{3}=V\left(u_{3}\right)+V\left(v_{3}\right) & \geqslant 2\left(\left|2 h_{1} g_{1}+\cdots+2 h_{k} g_{k}\right|+\left|2 h_{k+1} g_{k+1}+\cdots+2 h_{n} g_{n}+h_{p} g_{p}\right|\right) \\
& \geqslant 8 k(n-k) .
\end{aligned}
$$

Assuming $1 \leqslant k<n$, then it is straightforward to show that $8(n-1) \leqslant 8 k(n-k) \leqslant 2 n^{2}$. As a consequence, the vLF inequality $S_{3}$ is automatically violated when vLF inequality $S_{2}$ is, and does not need to be considered separately. 


\subsubsection{Single signal mode partition}

Finally, we consider partitions of a single signal mode, or several such, all belonging to different EPR pairs $(i,-i)$. Because the signal mode $a_{i}$ is highly entangled to the mode $a_{-i}$ and to all other signals by virtue of the preceding - the separability test is simple in the present case. It is straightforward to show that the necessary vLF inequality for the partition $\left.\left\{a_{1}, \ldots, a_{k}\right\}\left\{a_{-1}, \ldots, a_{-k},\left(a_{k+1}, a_{-(k+1)}\right), \ldots,\left(a_{n}, a_{-n}\right)\right)\right\}$,

$$
\begin{aligned}
S_{4}= & V\left(Q_{j}-Q_{-j}+\sum_{i \neq j} \frac{\alpha_{i}}{\alpha_{j}}\left(Q_{i}-Q_{-i}\right)\right)+V\left(\sum_{i \neq j} P_{i}+P_{-i}-\frac{\alpha_{i}}{\alpha_{j}}\left(P_{j}+P_{-j}\right)\right) \\
& \geqslant 2\left(\left|h_{1} g_{1}+\cdots+h_{k} g_{k}\right|+\left|h_{-1} g_{-1}+\ldots h_{-k} g_{-k}+2 h_{k+1} g_{k+1}+\ldots 2 h_{n} g_{n}\right|\right) \\
& \geqslant 2\left|-\sum_{i \neq 1} \frac{\alpha_{i}}{\alpha_{1}}+\sum_{i=2}^{k} \frac{\alpha_{k}}{\alpha_{1}}\right|+2\left|\sum_{i \neq 1} \frac{\alpha_{-i}}{\alpha_{1}}+\sum_{i=2}^{k} \frac{\alpha_{-k}}{\alpha_{1}}+0\right| \\
& \geqslant 4(n-k),
\end{aligned}
$$

is always violated in the presence of single EPR pair entanglement. This is because the left hand side term of Eq. (82) contains EPR nullifiers [48], a.k.a. EPR entanglement witnesses, whose squeezed variances tend toward zero. The inseparability of any other form of partitions on modes when modes $a_{1}$ and $a_{-1}$ are placed in different partitions, can be examined by inequalities similar to $S_{4}$ and with nonzero boundaries. Such inequalities are always vi-

olated. Therefore, if EPR entanglement is present (the checking of which is a staple of the experimental calibration of a regular two-mode squeezer), then the violation of both $S_{1}$ and $S_{2}$ is a necessary and sufficient condition to mode inseparability for all possible partitions in the optical frequency comb of a single OPO.

\subsection{Entanglement between pairs without considering the pump}

Here we ask the question of the possibility of multipartite entanglement between twins without considering the pump field. For that, we rewrite inequality $S_{2}$ without the pump quadratures:

$$
S_{2}^{\prime}=V\left(u_{2}^{\prime}\right)+V\left(v_{2}^{\prime}\right) \geqslant 8(n-1)
$$

with

$$
\begin{aligned}
u_{2}^{\prime} & =Q_{j+}+\sum_{i \neq j}^{n} \frac{\alpha_{i}}{\alpha_{j}} Q_{i+} \\
v_{2}^{\prime} & =\sum_{i \neq j}^{n} \frac{\alpha_{i}}{\alpha_{j}} P_{j+}-P_{i+} .
\end{aligned}
$$

In Fig. 5, we plot the violation of this inequality. As can be seen by comparing with Fig. 4, Inequality $S_{2}^{\prime}$ requires slightly larger $\sigma$ to be violated, compared to $S_{2}$, for small values of n. It shows we need to pump harder (and get closer to total depletion) in order to see pure entanglement between twin pairs. The arguments of subsections and 4.2.3 and 4.2.4 may be reused here to complete the inseparability proof. 


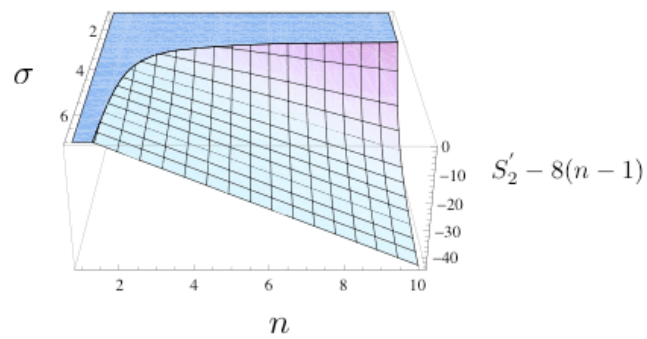

Fig. 5. Plot of the vLF inequality violation $S_{2}^{\prime}-8(n-1)$, versus $\sigma$, the pump to threshold power ratio, and $n$, the number of mode pairs inside the cavity, for $\Omega=0$ and $\alpha_{i}=\alpha_{j}, \forall i, j$.

\subsection{Optimal entanglement witnesses}

A valid question is whether the entanglement witnesses that were derived here on the basis of physical considerations, namely the pump-depletion-induced correlations between EPR pairs, are in fact the optimal entanglement witnesses for the system. In other words, do different observables exist that would lead to even more strongly violated vLF inequalities? Answering this question should, in turn, inform on what type of entangled state is really generated here. The search for optimal entanglement witnesses was addressed by Hyllus and Eisert, using semidefinite programming procedures [45]. While the scope of the present paper is limited to this successful demonstration of large-scale entanglement in a simple OPO, the exact nature of the quantum state generated is clearly an interesting followup question, on which light can be shed by seeking the optimal entanglement witnesses and checking whether they are different from the ones derived above.

\section{Conclusion}

We showed that a single OPO operating well above threshold can generate multipartite entanglement in its quantum optical frequency comb. We verified the multipartite nature of the entanglement by evaluating the van Loock-Furusawa separability criterion over all possible Qmode partitions. We showed that all of these vLF inequalities can be violated, for an arbitrary large number of pairs $n$, simply by increasing the input pump power higher above threshold.

While the presence of multipartite entanglement in such a simple system is a remarkable feature, it is important to keep in mind that the exact type of entanglement that is produced here (GHZ, W, cluster) is difficult to determine. The search for optimal entanglement witnesses for this system is a promising approach to illuminate this question. Note that previous work has shown that multipartite cluster-state generation, which was experimentally demonstrated in a single OPO below threshold [12], should actually fail above threshold [13]. However, the multipartite entanglement that we discovered in the simple OPO above threshold would certainly be useful for quantum communication applications, such as quantum secret sharing. 


\section{Acknowledgement}

This work was supported by U.S. National Science Foundation grants No. PHY-0855632 and No. PHY-0960047.

\section{References}

1. H. Yonezawa, T. Aoki, and A. Furusawa, "Demonstration of a quantum teleportation network for continuous variables," Nature 431, 430 (2004).

2. A. M. Lance, T. Symul, W. P. Bowen, B. C. Sanders, and P. K. Lam, "Tripartite quantum state sharing," Phys. Rev. Lett. 92, 177903 (2004).

3. H. J. Briegel and R. Raussendorf, "Persistent entanglement in arrays of interacting particles," Phys. Rev. Lett. 86, 910 (2001).

4. J. Zhang and S. L. Braunstein, "Continuous-variable Gaussian analog of cluster states," Phys. Rev. A 73, 032318 (2006).

5. R. Raussendorf and H. J. Briegel, "A one-way quantum computer," Phys. Rev. Lett. 86, 5188 (2001).

6. N. C. Menicucci, P. van Loock, M. Gu, C. Weedbrook, T. C. Ralph, and M. A. Nielsen, "Universal quantum computation with continuous-variable cluster states," Phys. Rev. Lett. 97, 110501 (2006).

7. N. D. Mermin, "From Cbits to Qbits: Teaching computer scientists quantum mechanics," American Journal of Physics 71, 23 (2003).

8. T. D. Ladd, F. Jelezko, R. Laflamme, Y. Nakamura, C. Monroe, and J. L. O'Brien, "Quantum computers," Nature 464, 45 (2010).

9. M. Greiner, O. Mandel, T. Esslinger, T. W. Hänsch, and I. Bloch, "Quantum phase transition from a superfluid to a Mott insulator in a gas of ultracold atoms," Nature 415, 39 (2002).

10. N. C. Menicucci, S. T. Flammia, and O. Pfister, "One-way quantum computing in the optical frequency comb," Phys. Rev. Lett. 101, 130501 (2008).

11. S. T. Flammia, N. C. Menicucci, and O. Pfister, "The optical frequency comb as a one-way quantum computer," J. Phys. B, 42, 114009 (2009).

12. M. Pysher, Y. Miwa, R. Shahrokhshahi, R. Bloomer, and O. Pfister, "Parallel generation of quadripartite cluster entanglement in the optical frequency comb," Phys. Rev. Lett. 107, 030505 (2011).

13. S. L. W. Midgley, M. K. Olsen, A. S. Bradley, and O. Pfister, "Analysis of a continuous-variable quadripartite cluster state from a single optical parametric oscillator," Phys. Rev. A 82, 053826 (2010).

14. R. C. Pooser and O. Pfister, "Observation of triply coincident nonlinearities in periodically poled $\mathrm{KTiOPO}_{4}$," Opt. Lett. 30, 2635 (2005).

15. M. Pysher, A. Bahabad, P. Peng, A. Arie, and O. Pfister, "Quasi-phase-matched concurrent nonlinearities in periodically poled $\mathrm{KTiPO}_{4}$ for quantum computing over the optical frequency comb," Opt. Lett. 35, 565 (2010).

16. R. Vyas and S. Singh, "Exact Quantum Distribution for Parametric Oscillators," Phys. Rev. Lett. 74, 2208 (1995).

17. K. Dechoum, P. Drummond, S. Chaturvedi, and M. Reid, "Critical quantum fluctuations in the nondegenerate parametric oscillator," Phys. Rev. A 70, 053807 (2004).

18. A. S. Bradley, M. K. Olsen, O. Pfister, and R. C. Pooser, "Bright tripartite entanglement in triply concurrent parametric down conversion," Phys. Rev. A 72, 053805 (2005).

19. S. L. W. Midgley, A. S. Bradley, O. Pfister, and M. K. Olsen, "Quadripartite continuous-variable entanglement via quadruply concurrent down-conversion," Phys. Rev. A 81, 063834 (2010).

20. C. Navarrete-Benlloch, E. Roldán, and G. J. de Valcárcel, "Noncritically Squeezed Light via Spontaneous Rotational Symmetry Breaking," Phys. Rev. Lett. 100, 203601 (2008).

21. C. Navarrete-Benlloch, A. Romanelli, E. Roldán, and G. J. de Valcárcel, "Noncritical quadrature squeezing in two-transverse-mode optical parametric oscillators," Phys. Rev. A 81, 043829 (2010).

22. C. Navarrete-Benlloch, G. J. de Valcárcel, and E. Roldán, "Generating highly squeezed hybrid 
Laguerre-Gauss modes in large-Fresnel-number degenerate optical parametric oscillators," Phys. Rev. A 79, 043820 (2009).

23. B. Chalopin, F. Scazza, C. Fabre, and N. Treps, "Multimode nonclassical light generation through the optical-parametric-oscillator threshold," Phys. Rev. A 81, 061804 (2010).

24. M. D. Reid and P. D. Drummond, "Correlations in nondegenerate parametric oscillation: Squeezing in the presence of phase diffusion," Phys. Rev. A 40, 4493 (1989).

25. J. Y. Courtois, A. Smith, C. Fabre, and S. Reynaud, "Phase diffusion and quantum noise in the optical parametric oscillator: a semiclassical approach," J. Mod. Opt. 38, 177 (1991).

26. P. van Loock and A. Furusawa, "Detecting genuine multipartite continuous-variable entanglement," Phys. Rev. A 67, 052315 (2003).

27. M. Reid, "Demonstration of the Einstein-Podolsky-Rosen paradox using nondegenerate parametric amplification," Phys. Rev. A 40, 913 (1989).

28. Z. Y. Ou, S. F. Pereira, H. J. Kimble, and K. C. Peng, "Realization of the Einstein-Podolsky-Rosen paradox for continuous variables," Phys. Rev. Lett. 68, 3663 (1992).

29. M. Reid and P. Drummond, "Quantum correlations of phase in nondegenerate parametric oscillation," Phys. Rev. Lett. 60, 2731 (1988).

30. A. Villar, L. S. Cruz, K. N. Cassemiro, M. Martinelli, and P. Nussenzveig, "Generation of Bright Two-Color Continuous Variable Entanglement," Phys. Rev. Lett. 95, 243603 (2005).

31. X. Su, A. Tan, X. Jia, Q. Pan, C. Xie, and K. Peng, "Experimental demonstration of quantum entanglement between frequency-nondegenerate optical twin beams," Opt. Lett. 31, 1133 (2006).

32. J. Jing, S. Feng, R. Bloomer, and O. Pfister, "Experimental continuous-variable entanglement from a phase-difference-locked optical parametric oscillator," Phys. Rev. A 74, 041804(R) (2006).

33. G. Keller, V. D'Auria, N. Treps, T. Coudreau, J. Laurat, and C. Fabre, "Experimental demonstration of frequency-degenerate bright EPR beams with a self-phase-locked OPO," Opt. Exp 16, 9351 (2008).

34. A. S. Villar, M. Martinelli, C. Fabre, and P. Nussenzveig, "Direct Production of Tripartite PumpSignal-Idler Entanglement in the Above-Threshold Optical Parametric Oscillator," Phys. Rev. Lett. 97, 140504 (2006).

35. A. S. Coelho, F. A. S. Barbosa, K. N. Cassemiro, A. S. Villar, M. Martinelli, and P. Nussenzveig, "Three-Color Entanglement," Science 326, 823 (2009).

36. K. N. Cassemiro and A. S. Villar, "Scalable continuous-variable entanglement of light beams produced by optical parametric oscillators," Phys. Rev. A 77, 022311 (2008).

37. O. Pfister, S. Feng, G. Jennings, R. Pooser, and D. Xie, "Multipartite continuous-variable entanglement from concurrent nonlinearities," Phys. Rev. A 70, 020302 (2004).

38. M. J. Collett and C. W. Gardiner, "Squeezing of intracavity and traveling-wave light fields produced in parametric amplification," Phys. Rev. A 30, 1386 (1984).

39. C. Gardiner and P. Zoller, Quantum Noise, A Handbook of Markovian and Non-Markovian Quantum Stochastic Methods with Applications to Quantum Optics, Springer Series in Synergetics, 3rd ed. (Springer, 2004).

40. S. Reynaud, C. Fabre, and E. Giacobino, "Quantum fluctuations in a two-mode parametric oscillator," J. Opt. Soc. Am. B 4, 1520 (1987).

41. L.-M. Duan, G. Giedke, J. Cirac, and P. Zoller, "Inseparability Criterion for Continuous Variable Systems," Phys. Rev. Lett. 84, 2722 (2000).

42. R. Simon, "Peres-Horodecki separability criterion for continuous variable systems," Phys. Rev. Lett. 84, 2726 (2000).

43. A. Peres, "Separability Criterion for Density Matrices," Phys. Rev. Lett. 77, 1413 (1996).

44. M. Horodecki, P. Horodecki, and R. Horodecki, "Separability of mixed states: necessary and sufficient conditions," Phys. Lett. A 223, 1 (1996).

45. P. Hyllus and J. Eisert, "Optimal entanglement witnesses for continuous-variable systems," New J. Phys. 8, 51 (2006).

46. B. Terhal, "Bell Inequalities and the Separability Criterion," Phys. Lett. A 271, 319 (2000).

47. M. Lewenstein, B. Kraus, J. I. Cirac, and P. Horodecki, "Optimization of entanglement witnesses," 
Phys. Rev. A 62, 052310 (2000).

48. M. Gu, C. Weedbrook, N. C. Menicucci, T. C. Ralph, and P. van Loock, "Quantum computing with continuous-variable clusters," Phys. Rev. A 79, 062318 (2009). 


\section{Appendix B}

\section{Experiment-Computer Interface: Data Acquisition and Data Analysis Techniques}

In order to improve data acquisition and data processing techniques and do real time data analysis, we decided to buy and implement Alazar ATS9440 waveform digitizer with a commercial real time sampling rate as fast as $125 \mathrm{M}$ sample/s. This waveform digitizer has 1 Gig dual-port memory and enabled us to acquire and analyze TES data real time for a live update on photon number statistics, correlation functions and histograms.

Extensive real time programming, dropped the actual real time sampling rate from the commercial $125 \mathrm{M}$ sample/s into $10 \mathrm{Msample/s}$. Overall we improved the data acquisition speed from $3 * 10^{4}$ in our old data acquision technique into $10^{7}$ samples per second using Alazar card. Implementing vectorized Matlab coding also helped us in speeding up the programming.

In the following, I included our Matlab programs for analyzing TES data in con- 
tinuous wave $(\mathrm{CW})$ regime. Analyzing TES data in $\mathrm{CW}$ regime is different from in pulse regime where all photons hit the detector at specific times and one can synchronize the photon counting window with the pump pulse repetition times. In $\mathrm{CW}$ regime photons are hitting the detector at random times and extra steps are required to recognize photon arrival times for digitizing data and calculating the correlation function, as was explained in section 2.2.

The main part of the Matlab code which reads TES data from Alazar card and analyzes them, is presented in the following.

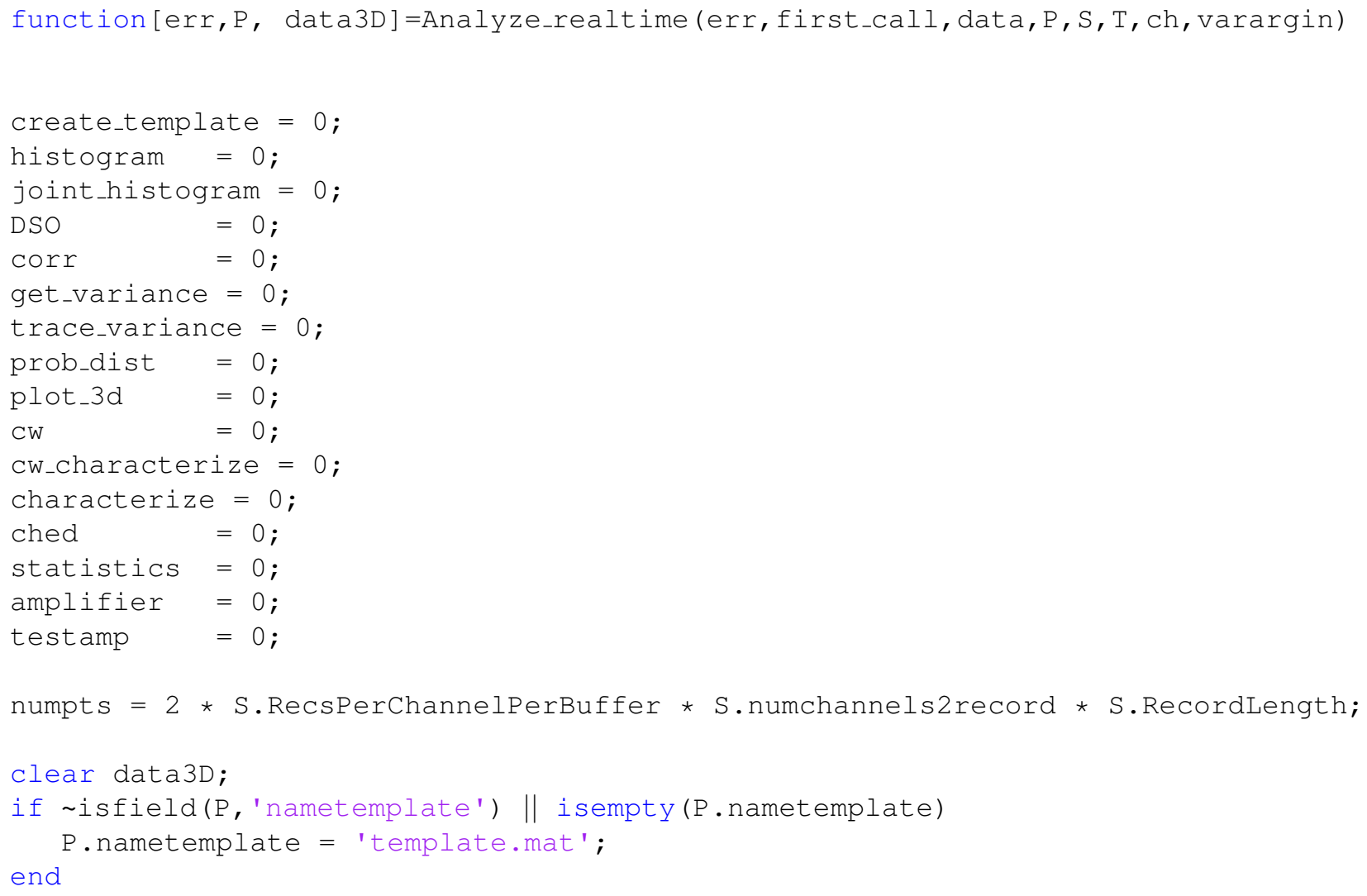




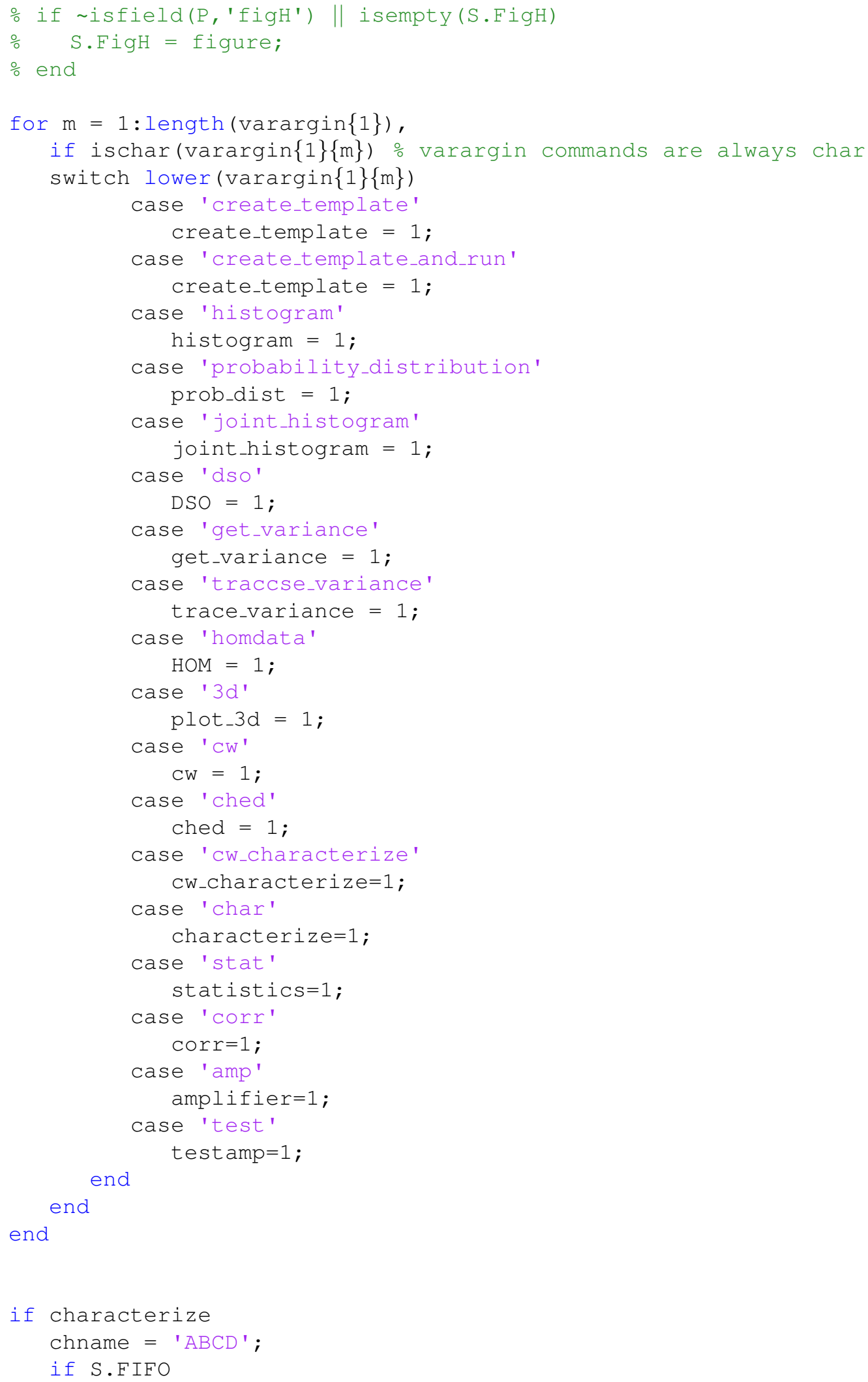


data3D = double (reshape (typecast (uint8 (data.Value (1:numpts)), 'uint16')

, S.numchannels2record, S.RecordLength,

S.RecsPerChannelPerBuffer)) - $2^{\wedge} 15$; else

data3D = double (reshape (typecast (uint8 (data.Value (1:numpts)), 'uint16'), S.RecordLength, S.numchannels2record, S.RecsPerChannelPerBuffer)) -2^15;

end

global call PhotonData;

data=data3D;

numchan=size (data, 1$)$;

size (data);

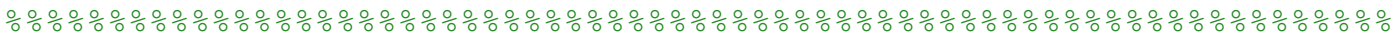

numtosum=6 . ;

mat $=(0:$ numtosum-1) ' ;

$\mathrm{L}=\mathrm{size}($ data, 2$)$;

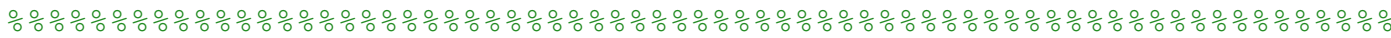

oto set the mean of the noise to zero, kill all photons above thresh then

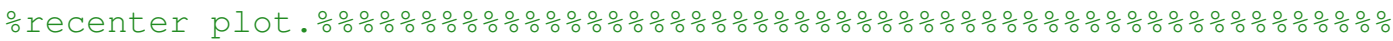
noisethresh $=[-150 ; 200 ; 200 ; 200]$; $\%$ threshold for noise mean to be $0 \ldots$

\% has to be decided by user

noisethresh=noisethresh ( 1 : numchan);

datai=data-repmat (noisethresh, 1, size (data, 2)) >0;

data=data-repmat ( (sum (data.*not (datai),2)/size (data,2)), 1, size (data, 2)) ;

noise $=0$

data $=$ data.$\star$ datai

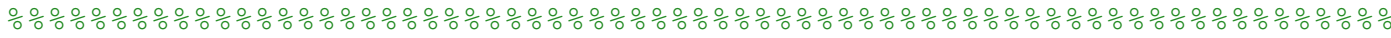

A NEW WAY OF CALCULATING PHOTON PEAKS... ONE THAT ALLOWS TIME BINS

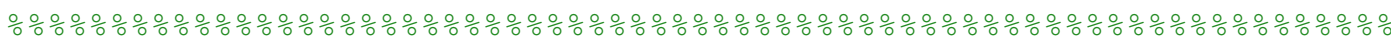

diffthresh $=[150 ; 150 ; 150 ; 150]$; $\%$ threshold to count a rising edge.

diffthresh=diffthresh ( 1 : numchan);

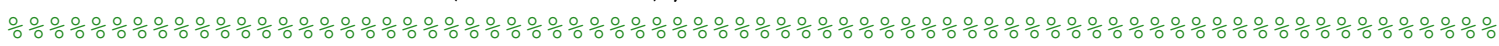

\%change to get 4 channels $\% \% \frac{0}{0} \%$

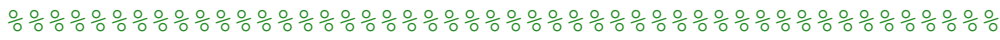

dataf=zeros (size (data));

for $\mathrm{k}=1$ : numchan

phindex=find (diff $(\operatorname{diff}(\operatorname{data}(\mathrm{k},:))) \operatorname{diffthresh}(\mathrm{k}))>0)$;

ophoton indices at rising edge

$\mathrm{d}=\operatorname{data}(\mathrm{k},:)$;

$\mathrm{df}=\operatorname{dataf}(\mathrm{k},:)$;

datajj=repmat (mat, 1, size (phindex, 2)) +repmat (phindex, numtosum, 1) +1;

ocount numtosum points after first point

size (datajj);

datajj(datajj>length $(\mathrm{d})$ ) =1; \%if last photons longers than datasize truncate if $\operatorname{size}(\operatorname{datajj,2)==1}$

end

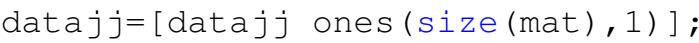

datatt $=d(\operatorname{datajj)}$;

size (phindex);

phmax $=\max ($ datatt $,[], 1)-\max (0, \min (\operatorname{datatt},[], 1)-50)$;

otake the max point at each photon peak 
phmax=sum(datatt, 1$)$; otake the max point at each photon peak size (phmax);

df $($ phindex $)=$ phmax; oset the photon heights to photon indices, $\operatorname{dataf}(k,:)=\operatorname{df}$;

end

PhotonData $=[$ PhotonData dataf $]$;

for $\mathrm{k}=1$ : numchan

subplot $(2,2, \mathrm{k})$;

$[\mathrm{n}, \mathrm{x}]=$ hist $($ PhotonData, 400$)$;

stairs $(\mathrm{x}, \mathrm{n})$;

set (gca,' 'yscale', 'log');

end

lastcall $=S$. Repeats*S.numBuffers;

$\mathrm{cal}=\mathrm{call}$;

$\mathrm{PBL}=-2000$ * (ones (numchan, 1));

$\mathrm{PBH}=\mathrm{inf} *($ ones (numchan, 1$))$;

if $\operatorname{call}==$ lastcall

fprintf('Click at the boundry of peaks. Press enter when finished. $\left.\backslash n^{\prime}\right)$

$[\mathrm{PB}, \mathrm{PBy}]=\mathrm{ginput}$

$\mathrm{PB}=$ reshape $(\mathrm{PB}$, length $(\mathrm{PB}) / \mathrm{S}$. numchannels2record, S. numchannels2record) ';

$\mathrm{PB}=\left[\begin{array}{lll}\mathrm{PBL} & \mathrm{PB} & \mathrm{PBH}\end{array}\right]$;

save ('photon_limits', 'PB' )

end

$\operatorname{call}=\operatorname{call}+1$

drawnow;

end

if $\mathrm{CW}$

\% when FIFO, structure: ABCDABCDABCD 응 otherwise AAAAABBBBBCCCCCDDDDD

- read data from buffer

chname $=$ 'ABCD';

if S.FIFO

data3D = double (reshape (typecast (uint8 (data.Value (1:numpts)), 'uint16'),

S.numchannels2record, S.RecordLength, S.RecsPerChannelPerBuffer)) - 2^15;

else

data3D = double(reshape (typecast (uint8 (data.Value (1:numpts)), 'uint16'),

S.RecordLength, S.numchannels2record, S.RecsPerChannelPerBuffer)) -2^15;

end

global photons Ns cs scaled_peaksfs $\mathrm{Nm} \mathrm{cm}$ Nh numchan

global ntotal

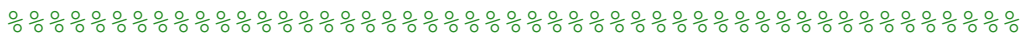

datasum=sum (data3D, 1); 


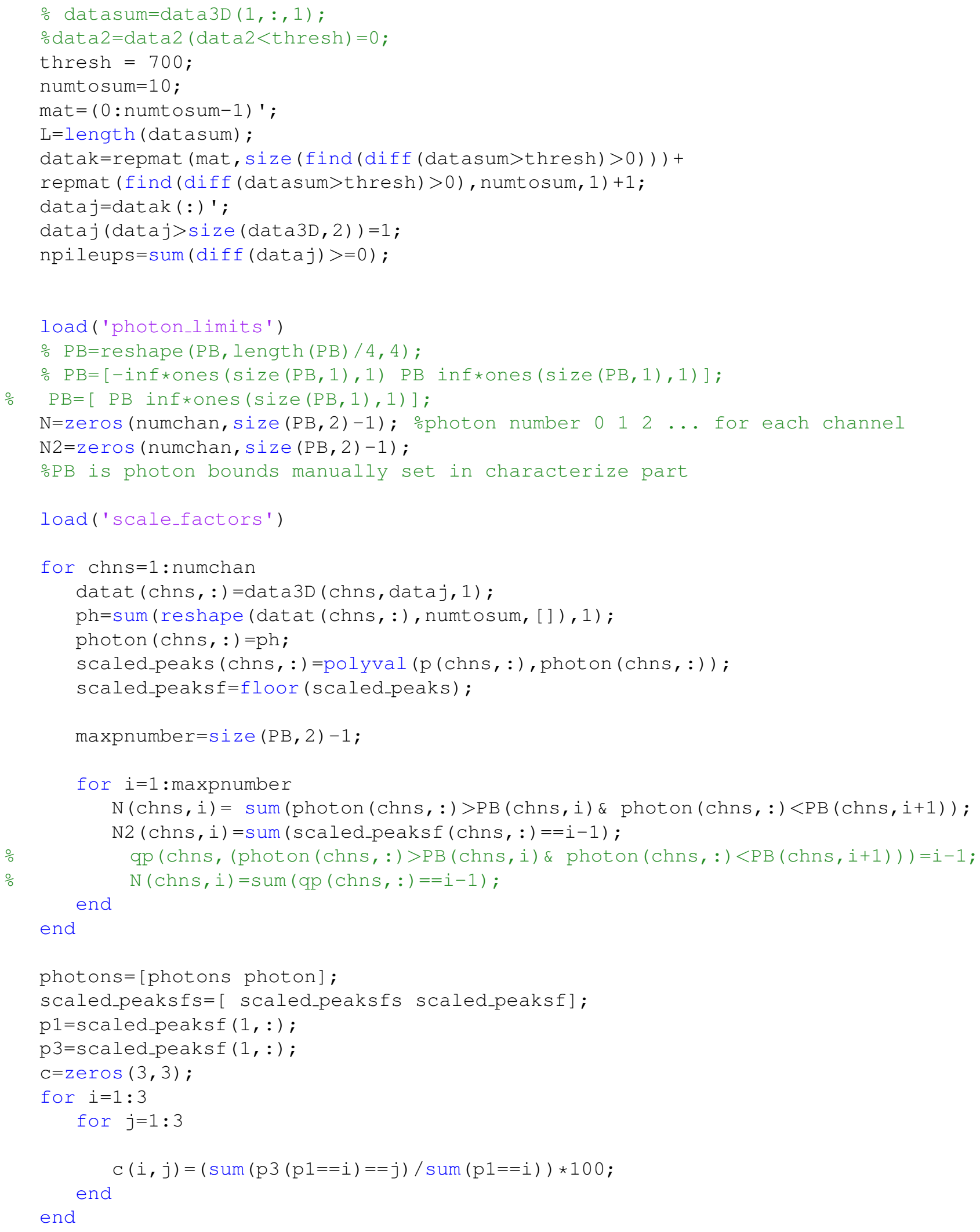




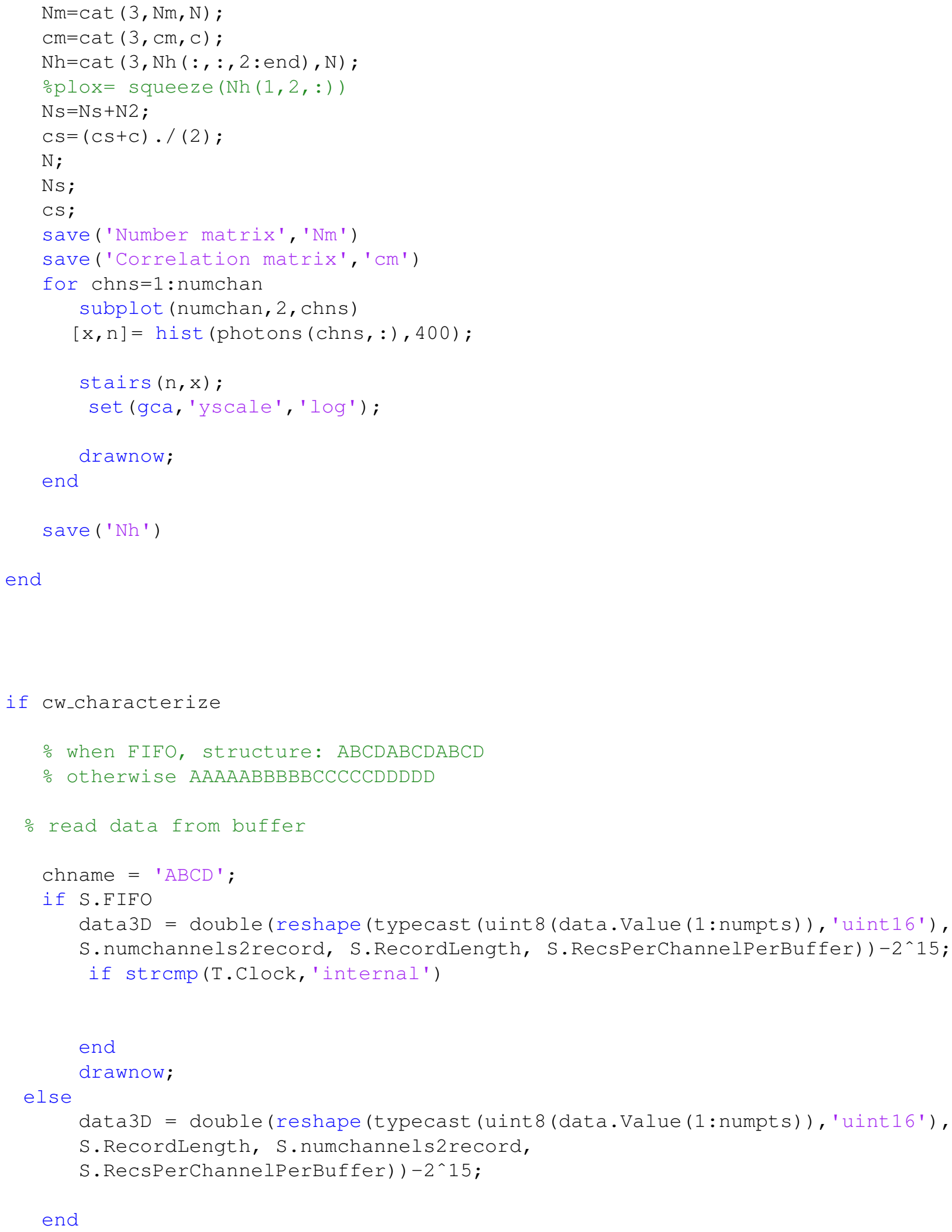




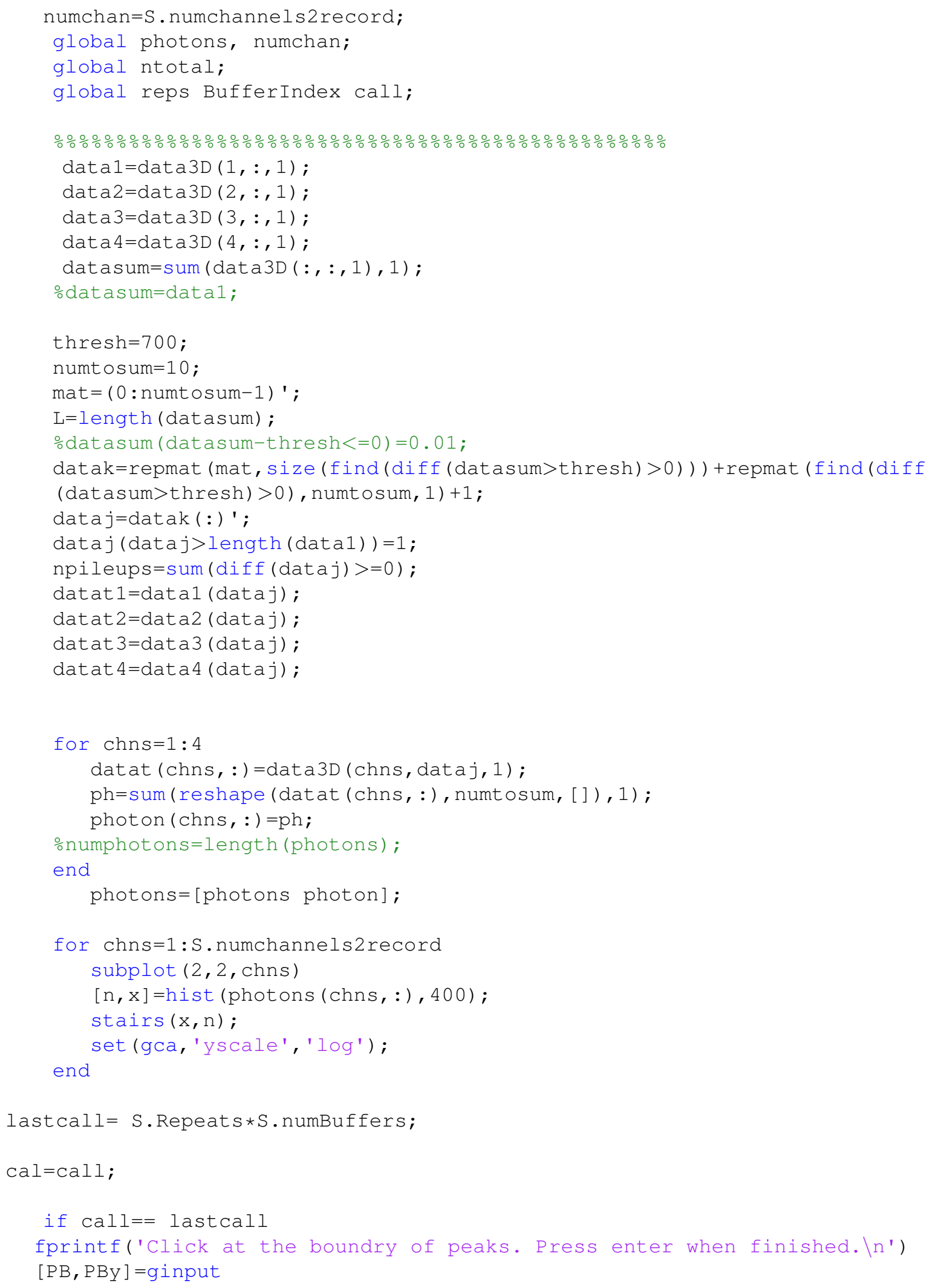




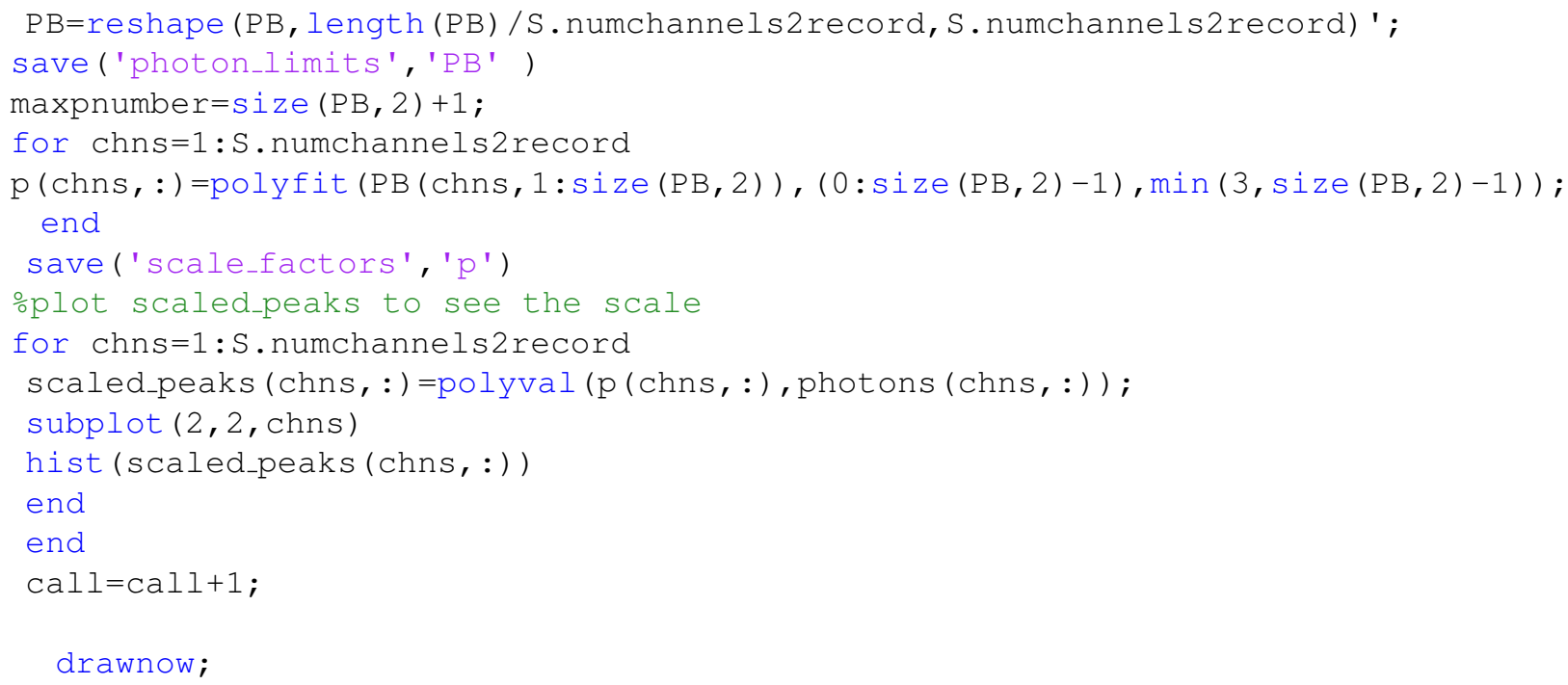




\section{Bibliography}

[1] D. F. Walls and G. J. Milburn, Quantum Optics (Springer, Berlin, 1994).

[2] R. H. Brown and R. Q. Twiss, "Interferometry of the intensity fluctuations in light. i. basic theory: The correlation between photons in coherent beams of radiation," Proc. Roy. Soc, A p. 242:300 (1957).

[3] R. H. Brown and R. Q. Twiss, "Interferometry of the intensity fluctuations in light ii. an experimental test of the theory for partially coherent light," Proc. Roy. Soc. A p. 243:291 (1958).

[4] Z. Y. Ou, "Fundamental quantum limit in precision phase measurement," Phys. Rev. A 55, 2598-2609 (1997).

[5] C. Caves, "Quantum-mechanical radiation-pressure fluctuations in an interferometer," Phys. Rev. Lett. 45, 75 (1980).

[6] B. M. Escher, R. L. de Matos Filho, and L. Davidovich, "General framework for estimating the ultimate precision limit in noisy quantum-enhanced metrology," Nat Phys 7, 406-411 (2011). 
[7] V. Giovannetti, S. Lloyd, and L. Maccone, "Quantum metrology," Phys. Rev. Lett. 96, 010401 (2006).

[8] A. Luis and L. Sánchez-Soto, "Quantum phase difference, phase measurements and Stokes operators," Prog. Opt. 41, 421 (2000).

[9] T. Kim, O. Pfister, M. J. Holland, J. Noh, and J. L. Hall, "Influence of decorrelation on Heisenberg-limited interferometry with quantum correlated photons," Phys. Rev. A 57, 4004-4013 (1998).

[10] J. F. Clauser and A. Shimony, "Bell's theorem. experimental tests and implications," Reports on Progress in Physics 41, 1881 (1978).

[11] A. Aspect, P. Grangier, and G. Roger, "Experimental realization of EinsteinPodolsky-Rosen-Bohm gedankenexperiment: A new violation of Bell's inequalities," Phys. Rev. Lett. 49, 91-94 (1982).

[12] P. G. Kwiat, K. Mattle, H. Weinfurter, A. Zeilinger, A. V. Sergienko, and Y. Shih, "New high-intensity source of polarization-entangled photon pairs," Phys. Rev. Lett. 75, 4337-4341 (1995).

[13] J. C. Howell, A. Lamas-Linares, and D. Bouwmeester, "Experimental violation of a spin-1 Bell inequality using maximally entangled four-photon states," Phys. Rev. Lett. 88, 030401 (2002). 
[14] P. Shadbolt, J. C. F. Mathews, A. Laing, and J. L. O’Brien, "Testing foundations of quantum mechanics with photons," Nat Phys 10, 278-286 (2014).

[15] C. H. Bennett, F. Bessette, G. Brassard, L. Salvail, and J. A. Smolin, "Experimental quantum cryptography," J. Cryptology 5, 3-28 (1992).

[16] C. H. Bennett, "Quantum cryptography using any two nonorthogonal states," Phys. Rev. Lett. 68, 3121-3124 (1992).

[17] Antonio, N. Brunner, N. Gisin, S. Massar, S. Pironio, and V. Scarani, "Deviceindependent security of quantum cryptography against collective attacks," Phys. Rev. Lett. 98, 230501 (2007).

[18] N. Sangouard and H. Zbinden, "What are single photons good for?" Journal of Modern Optics 59, Issue 17, 1458-1464.

[19] C. Simon, H. de Riedmatten, M. Afzelius, N. Sangouard, H. Zbinden, and N. Gisin, "Quantum repeaters with photon pair sources and multimode memories," Phys. Rev. Lett. 98, 190503 (2007).

[20] L.-M. Duan, M. D. Lukin, J. I. Cirac, and P. Zoller, "Long-distance quantum communication with atomic ensembles and linear optics," Nature 414, 413-418 (2001).

[21] E. Knill, R. Laflamme, and G. J. Milburn, "A scheme for efficient quantum computation with linear optics," Nature 409, 46-52 (2001). 
[22] S. D. Bartlett, B. C. Sanders, S. L. Braunstein, and K. Nemoto, "Efficient classical simulation of continuous variable quantum information processes," Phys. Rev. Lett. 88, 097904 (2002).

[23] J. Eisert, S. Scheel, and M. B. Plenio, "Distilling Gaussian states with Gaussian operations is impossible," Phys. Rev. Lett. 89, 137903 (2002).

[24] J. Niset, J. Fiurášek, and N. J. Cerf, "No-go theorem for Gaussian quantum error correction," Phys. Rev. Lett. 102, 120501 (2009).

[25] D. Gottesman, A. Kitaev, and J. Preskill, "Encoding a qubit in an oscillator," Phys. Rev. A 64, 012310 (2001).

[26] N. C. Menicucci, "Fault-tolerant measurement-based quantum computing with continuous-variable cluster states," Phys. Rev. Lett. 112, 120504 (2014).

[27] D. Magde and H. Mahr, "Study in ammonium dihydrogen phosphate of spontaneous parametric interaction tunable from 4400 to 16000 ," Phys. Rev. Lett. 18, 905-907 (1967).

[28] D. C. Burnham and D. L. Weinberg, "Observation of simultaneity in parametric production of optical photon pairs," Phys. Rev. Lett. 25, 84-87 (1970).

[29] A. I. Lvovsky, H. Hansen, T. Aichele, O. Benson, J. Mlynek, and S. Schiller, "Quantum state reconstruction of the single-photon Fock state," Phys. Rev. Lett. 87, 050402 (2001). 
[30] J. E. S. Marco Fiorentino, Paul L. Voss and P. Kumar, "All-fiber photon-pair source for quantum communications," IEEE Photonics Technology Letters 14 (2002).

[31] A. R. McMillan, J. Fulconis, M. Halder, C. Xiong, J. G. Rarity, and W. J. Wadsworth, "Narrowband high-fidelity all-fibre source of heralded single photons at 1570 nm," Optics Express 17, 6156-6165 (2009).

[32] P. Michler, A. Kiraz, C. Becher, W. V. Schoenfeld, P. M. Petroff, L. Zhang, E. Hu, and A. Imamoglu, "A quantum dot single-photon turnstile device," Science 290, $2282-2285(2000)$.

[33] F. Diedrich and H. Walther, "Nonclassical radiation of a single stored ion," Phys. Rev. Lett. 58, 203-206 (1987).

[34] H. J. Kimble, M. Dagenais, and L. Mandel, "Photon antibunching in resonance fluorescence," Phys. Rev. Lett. 39, 691-695 (1977).

[35] C. Kurtsiefer, S. Mayer, P. Zarda, and H. Weinfurter, "Stable solid-state source of single photons," Phys. Rev. Lett. 85, 290-293 (2000).

[36] B. G. Christensen, K. T. McCusker, J. B. Altepeter, B. Calkins, T. Gerrits, A. E. Lita, A. Miller, L. K. Shalm, Y. Zhang, S. W. Nam, N. Brunner, C. C. W. Lim, N. Gisin, and P. G. Kwiat, "Detection-loophole-free test of quantum nonlocality, and applications," Phys. Rev. Lett. 111, 130406 (2013). 
[37] Giustina, Marissa, Mech, Alexandra, R. J. Beyer, A. Lita, B. Calkins, T. Gerrits, S. W. Nam, R. Ursin, and A. Zeilinger, "Bell violation using entangled photons without the fair-sampling assumption," Nature 497, 227-230 (2013).

[38] A. E. Lita, A. J. Miller, and S. W. Nam, "Counting near-infrared single-photons with 95\% efficiency," Opt. Express 16, 3032-3040 (2008).

[39] R. S. Bennink, "Optimal collinear Gaussian beams for spontaneous parametric down-conversion," Phys. Rev. A 81, 053805 (2010).

[40] R. W. Boyd, Nonlinear Optics (Academic Press, 2008), 3rd ed.

[41] M. Born and E. Wolf, Principles of Optics: Electromagnetic Theory of Propagation, Interference and Diffraction of Light (Cambridge University Press, 1882-1970).

[42] Z. Y. Ou and Y. J. Lu, "Cavity enhanced spontaneous parametric downconversion for the prolongation of correlation time between conjugate photons," Phys. Rev. Lett. 83, 2556-2559 (1999).

[43] M. J. Collett and C. W. Gardiner, "Squeezing of intracavity and traveling-wave light fields produced in parametric amplification," Phys. Rev. A 30, 1386-1391 (1984).

[44] D. Walls and G. Milburn, Quantum Optics (Springer, 1994). 
[45] H.-A. Bachor and T. C. Ralph, A Guide to Experiments in Quantum Optics, 2nd, Revised and Enlarged Edition (Wiley-VCH, March 2004).

[46] L.-A. Wu, H. J. Kimble, J. L. Hall, and H. Wu, "Generation of squeezed states by parametric down conversion," Phys. Rev. Lett. 57, 2520 (1986).

[47] Z. Y. Ou, S. F. Pereira, H. J. Kimble, and K. C. Peng, "Realization of the Einstein-Podolsky-Rosen paradox for continuous variables," Phys. Rev. Lett. 68, 3663 (1992).

[48] S. Feng and O. Pfister, "Stable nondegenerate optical parametric oscillation at degenerate frequencies in Na:KTP," J. Opt. B: Quantum Semiclass. Opt. 5, 262 (2003).

[49] D. Lee and N. C. Wong, "Stabilization and tuning of a doubly resonant optical parametric oscillator," J. Opt. Soc. Am. B 10, 1659 (1993).

[50] J. H. Shapiro and N. C. Wong, "An ultrabright narrowband source of polarization-entangled photon pairs," Journal of Optics B: Quantum and Semiclassical Optics Opt. 2 L1.

[51] C. E. Kuklewicz, E. Keskiner, F. N. C. Wong, and J. H. Shapiro, "A high-flux entanglement source based on a doubly resonant optical parametric amplifier," J. Opt. B: Quantum Semiclass. Opt. 4, S162-S168 (2002). 
[52] S. Ramelow, A. Mech, M. Giustina, S. Grblacher, W. Wieczorek, J. Beyer, A. Lita, . S. W. N. A. Z. Brice Calkins, 6 Thomas Gerrits, and R. Ursin .

[53] M. D. C. Pereira, F. E. Becerra, B. L. Glebov, J. Fan, S. W. Nam, and A. Migdall, "Demonstrating highly symmetric single-mode, single-photon heralding efficiency in spontaneous parametric downconversion," Opt. Lett. 38, 1609$1611(2013)$.

[54] D. H. Smith, G. Gillett, M. P. de Almeida, C. Branciard, A. Fedrizzi, T. J. Weinhold, A. Lita, B. Calkins, T. Gerrits, H. M. Wiseman, S. W. Nam, and A. G. White, "Conclusive quantum steering with superconducting transitionedge sensors," Nat. Commun. 3, 625 (2012).

[55] C. M. Caves, "Quantum-mechanical noise in an interferometer," Phys. Rev. D 23, 1693-1708 (1981).

[56] B. Yurke, S. L. McCall, and J. R. Klauder, "SU(2) and SU(1,1) interferometers," Phys. Rev. A 33, 4033-4054 (1986).

[57] M. J. Holland and K. Burnett, "Interferometric detection of optical phase shifts at the Heisenberg limit," Phys. Rev. Lett. 71, 1355-1358 (1993).

[58] "A gravitational wave observatory operating beyond the quantum shot-noise limit," Nat Phys 7, 962-965 (2011). 
[59] "Enhanced sensitivity of the ligo gravitational wave detector by using squeezed states of light," Nat Photon 7, 613-619 (2013).

[60] L. C. Biedenharn and van Ham, Quantum Theory of Angular Momentum. (1965).

[61] L. C. Biedenharn and J. D. Louck, "Angular momentum in quantum physics: Theory and application," Addison-Wesley (1981).

[62] C. W. Gardiner, "Quantum noise," Springer-Verlag, Berlin p. 27 (1991).

[63] G. P. Agrawal, Fiber-Optic Communication Systems (Wiley Series in Microwave and Optical Engineering,, Wiley- Interscience, Hoboken, NJ, 2010), 4th ed.

[64] C. W. Helstrom, Quantum Detection and Estimation Theory (Mathematics in Science and Engineering, 123,(Academic Press, New York), 1976).

[65] S. L. Braunstein and P. van Loock, "Quantum information with continuous variables," Rev. Mod. Phys. 77, 513-577 (2005).

[66] S. Olivares and M. G. A. Paris, "Binary optical communication in single-mode and entangled quantum noisy channels," Journal of Optics B: Quantum and Semiclassical Optics Opt. 669 (2004).

[67] R. Nair, B. J. Yen, S. Guha, J. H. Shapiro, and S. Pirandola, "Symmetric mary phase discrimination using quantum-optical probe states," Phys. Rev. A $\mathbf{8 6}$, $022306(2012)$. 
[68] S. Pirandola, "Quantum reading of a classical digital memory," Phys. Rev. Lett. 106, 090504 (2011).

[69] R. Nair, "Discriminating quantum-optical beam-splitter channels with numberdiagonal signal states: Applications to quantum reading and target detection," Phys. Rev. A 84, 032312 (2011).

[70] P. Hausladen, R. Jozsa, B. Schumacher, M. Westmoreland, and W. K. Wootters, "Classical information capacity of a quantum channel," Phys. Rev. A 54, 1869$1876(1996)$.

[71] A. S. Holevo, Problemy Peredachi lnformatsii 15, 3 [Problems of lnformation Transmission (USSR) 15, 247 (1979).

[72] S. Guha and J. H. Shapiro, "Reading boundless error-free bits using a single photon," Phys. Rev. A 87, 062306 (2013).

[73] J. Wang, J.-Y. Yang, I. M. Fazal, N. Ahmed, Y. Yan, H. Huang, Y. Ren, Y. Yue, S. Dolinar, M. Tur, and A. E. Willner, "Terabit free-space data transmission employing orbital angular momentum multiplexing," Nat. Photon. pp. 488-496 (2012).

[74] M. Zukowski, A. Zeilinger, and M. A. Horne, "Realizable higher-dimensional twoparticle entanglements via multiport beam splitters," Phys. Rev. A 55, 2564$2579(1997)$. 
[75] A. Peruzzo, A. Laing, A. Politi, T. Rudolph, and J. L. O’Brien, "Multimode quantum interference of photons in multiport integrated devices," Nature Communications 2, 224 (2011). 